\title{
ULTRAFAST OPTICAL
}

\author{
OF \\ PHOTONIC CRYSTALS
}

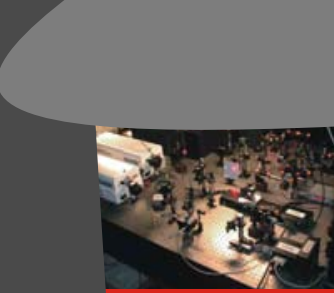

Tijmen Euser 



\section{ULTRAFAST OPTICAL SWITCHING OF PHOTONIC CRYSTALS}


Promotiecommissie:

Promotor: $\quad$ prof. dr. W. L. Vos

Overige leden: prof. dr. J. I. Dijkhuis

prof. dr. L. Kuipers

prof. dr. A. Lagendijk

prof. dr. M. Wegener

The work described in this thesis is part of the research program of the "Stichting Fundamenteel Onderzoek der Materie" (FOM), which is financially supported by the

“Nederlandse Organisatie voor Wetenschappelijk Onderzoek (NWO)”.

This work was carried out at the

Complex Photonic Systems Group,

Faculty of Science and Technology and MESA ${ }^{+}$Research Institute for Nanotechnology,

University of Twente, P.O. Box 217, 7500AE Enschede, The Netherlands,

and at the

FOM Institute for Atomic and Molecular Physics

Kruislaan 407, 1098SJ Amsterdam, The Netherlands,

where a limited number of copies of this thesis is available.

This thesis can be downloaded from www.photonicbandgaps.com.

Printed by Print Partners Ipskamp, Enschede, The Netherlands (2007).

ISBN 978-90-365-2471-1 


\title{
ULTRAFAST OPTICAL SWITCHING OF PHOTONIC CRYSTALS
}

\author{
PROEFSCHRIFT \\ ter verkrijging van \\ de graad van doctor aan de Universiteit Twente, \\ op gezag van de rector magnificus, \\ prof. dr. W. H. M. Zijm, \\ volgens besluit van het College voor Promoties \\ in het openbaar te verdedigen \\ op vrijdag 2 maart 2007 om 15.00 uur
}

door

Tijmen Godfried Euser

geboren op 16 juli 1978

te Wageningen 
Dit proefschrift is goedgekeurd door:

prof. dr. W. L. Vos 
Aan mijn ouders 



\section{Contents}

1 Introduction 13

1.1 Photonic crystals . . . . . . . . . . . . . . . . 13

1.1 .1 Intro . . . . . . . . . . . . . . . 13

1.1.2 Bragg diffraction in photonic crystals . . . . . . . . . . . . 14

1.1.3 Photonic strength . . . . . . . . . . . . . . . 15

1.1.4 3D photonic band gaps . . . . . . . . . . . . . . 17

1.1.5 Photonic band gap crystals . . . . . . . . . . . . 17

1.2 Switching photonic crystals . . . . . . . . . . . . 18

1.2.1 Density of states switching . . . . . . . . . . 18

1.2.2 Propagation switching . . . . . . . . . . . . 20

1.2.3 Relevant timescales ................. 20

2 Experimental considerations $\quad 23$

2.1 Experimental requirements . . . . . . . . . . . . . . . 23

2.2 Switching mechanisms ................... 24

2.2 .1 Introduction . . . . . . . . . . . . . . . 24

2.2.2 Free carrier excitation . . . . . . . . . . . . 25

2.2 .3 Optimal conditions . . . . . . . . . . . . . 26

3 Spatial homogeneity of switched photonic crystals and of bulk semiconductors 29

3.1 Introduction . . . . . . . . . . . . . . . . . . . 29

3.2 Optical properties of silicon . . . . . . . . . . . . . . . . 31

3.3 Homogeneity of switched semiconductors . . . . . . . . . . . . . 31

3.3.1 Theoretical model . . . . . . . . . . . . . . . . 31 
3.3.2 Homogeneity plot . . . . . . . . . . . . . . . . . . . 34

3.4 Switching homogeneity in real photonic crystals . . . . . . . . . . 39

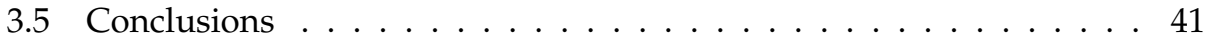

4 Instrumentation 43

4.1 Pump probe setup . . . . . . . . . . . . . . . . 43

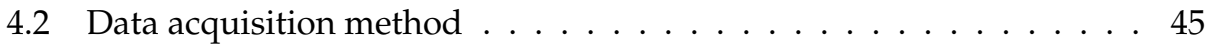

4.3 Ultrafast switching of a bulk Si wafer . . . . . . . . . . . . . . 50

4.3.1 Temporal behavior . . . . . . . . . . . . . . . 50

4.3.2 Lateral homogeneity . . . . . . . . . . . . . . . . 53

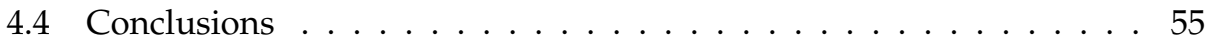

5 Ultrafast switching of the photonic strength of III-V semiconductor Bragg reflectors $\quad 57$

5.1 Introduction . . . . . . . . . . . . . . . . . 57

5.2 Sample . . . . . . . . . . . . . . . . . . . 59

5.2.1 GaAs/AlAs Distributed Bragg Reflectors . . . . . . . . . . 59

5.2.2 Linear reflectivity spectra . . . . . . . . . . . . . . . 59

5.2 .3 Photonic strength . . . . . . . . . . . . . . . . . . 60

5.2 .4 Switching experiment $\ldots \ldots \ldots \ldots 1$

5.3 Ultrafast switching of the photonic strength . . . . . . . . . . . . 61

5.3.1 Differential reflectivity spectra $\ldots \ldots \ldots \ldots 1$

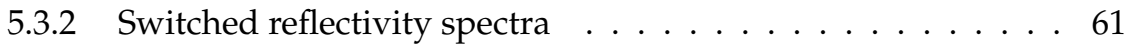

5.3.3 Irradiance scaling of the photonic strength . . . . . . . . 65

5.3.4 Spatial homogeneity . . . . . . . . . . . . 66

5.3.5 Temporal evolution of the reflectivity . . . . . . . . . 67

5.4 Theoretical model of switched DBR samples . . . . . . . . . . . 69

5.4 .1 Transfer matrix theory . . . . . . . . . . . . . . . . 69

5.4 .2 Optimization procedure . . . . . . . . . . . . . 69

5.4.3 Spectral comparison between measurement and theory . . . 70

5.4 .4 Induced absorption . . . . . . . . . . . . 70

5.4.5 Changes in photonic strength and center frequency . . . . . 70

5.5 Conclusions and outlook . . . . . . . . . . . . . . . . . 71

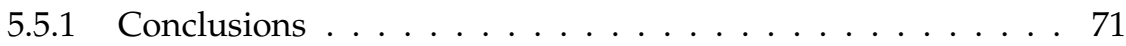

5.5 .2 Outlook ..................... 72 
6 Ultrafast switching of Si woodpile photonic band gap crystals 73

6.1 Introduction . . . . . . . . . . . . . . . 73

6.2 Si woodpile photonic band gap crystals . . . . . . . . . . . . . 74

6.3 Linear reflectivity spectra . . . . . . . . . . . . . 77

6.3.1 Stop band at normal incidence . . . . . . . . . . . . 77

6.3.2 Discussion of the nature of the observed stop band . . . . . 80

6.4 Comparison to exact modal method theory . . . . . . . . . . . . . 80

6.5 Experimental results . . . . . . . . . . . . . . . . . 83

6.5 .1 Stop band shift .................... 83

6.5.2 Differential reflectivity versus probe delay and wavelength . 84

6.5.3 Time-resolved measurements . . . . . . . . . . . . 87

6.5 .4 Free-carrier effects . . . . . . . . . . . . . . . 88

6.5 .5 Electronic Kerr effect . . . . . . . . . . . . . . . . . . . 91

6.6 Discussion . . . . . . . . . . . . . . . . . 93

6.6.1 Comparison of measured and calculated linear reflectivity . . 94

6.6.2 Induced absorption in the switched crystal . . . . . . . . . . 95

6.6.3 The 'switching requirements' .............. 96

6.6.4 Asymmetry in the stop band shift . . . . . . . . . . . . 97

6.7 Conclusions and outlook . . . . . . . . . . . . . . . . . 97

7 Ultrafast switching of Si inverse opals 99

7.1 Introduction . . . . . . . . . . . . . . . . 99

7.2 Sample description . . . . . . . . . . . . . . . 100

7.2.1 Fabrication method . . . . . . . . . . . . . . . 100

7.2.2 Structural analysis . . . . . . . . . . . . . . . 101

7.3 Linear reflectivity measurements . . . . . . . . . . . . . . . . . . . . . . . . . . . . . . . . . .

7.3.1 FT-IR reflectivity measurements . . . . . . . . . . . . 103

7.3.2 Laser reflectivity measurements . . . . . . . . . . . . . . . . 104

7.3.3 Extended frequency range interferometry . . . . . . . . . . 106

7.3.4 Photonic band structure . . . . . . . . . . . . . . . . . 107

7.4 Pump-probe reflectivity measurements . . . . . . . . . . . . . . . 109

7.4.1 Differential reflectivity . . . . . . . . . . . . . 109

7.4.2 Switched spectra at fixed probe delay . . . . . . . . . . . 110

7.4.3 Switching time traces . . . . . . . . . . . . 112

7.4.4 Induced probe absorption . . . . . . . . . . . . . . . 113

7.4 .5 Irradiance scaling . . . . . . . . . . . . . . . . 115

7.4.6 Comparison to band structure . . . . . . . . . . 116

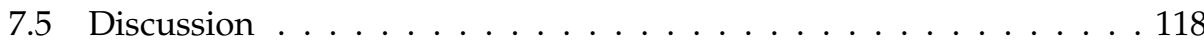


7.5.1 Carrier density . . . . . . . . . . . . . . . . . . . . . 119

7.5.2 Two-photon absorption coefficient . . . . . . . . . . . . . 119

7.5 .3 Switched density of states . . . . . . . . . . . . . 120

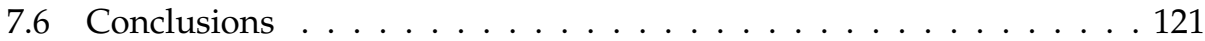

8 Recommendations 123

8.1 Applications . . . . . . . . . . . . . . . . . . 123

8.2 Adiabatic and non-adiabatic switching . . . . . . . . . . . . . 124

8.3 Switched lifetime measurements with ultrafast shutters . . . . . . . 124

8.4 Non-monotonic effects due to Kerr and free carriers . . . . . . . . . 125

8.5 Z-scan experiments on poly silicon samples . . . . . . . . . . 126

8.6 Samples fabricated by etching techniques . . . . . . . . . 126

$\begin{array}{ll}\text { A Drude description of excited semiconductors } & 127\end{array}$

A.1 Introduction . . . . . . . . . . . . . . . . . . . . 127

A.2 Basic assumptions . . . . . . . . . . . . . . . . 127

A.3 Drude model for free carriers in $\mathrm{Si} \ldots \ldots \ldots \ldots$

A.4 Drude description of excited carriers in GaAs . . . . . . . . . . 130

B WPC model 133

B.1 Solving Maxwell's equation for a photonic crystal by Fourier expansion133

B.2 The Exact Model Method (EMM) . . . . . . . . . . . . . . . . 134

B.3 Advantages and limitations of the EMM algorithm . . . . . . . . . 135

B.4 Test on 1D Bragg stack with comparison to exact results . . . . . . 136

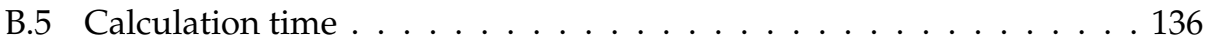

B.6 Convergence test for a 3D woodpile crystal . . . . . . . . . 137

$\begin{array}{lr}\text { C Z-scan measurements } & 139\end{array}$

C.1 Introduction . . . . . . . . . . . . . . . . . . . . . . . . 139

C.1.1 Experimental . . . . . . . . . . . . . . . . . . . 140

C.1.2 Model . . . . . . . . . . . . . . . . . . . . 141

C.2 Results . . . . . . . . . . . . . . . . . . . . 142

C.2.1 Two-photon absorption in $\mathrm{Si} \ldots \ldots \ldots 142$

C.2.2 Two-photon absorption in GaAs . . . . . . . . . . . . . . 142

C.2.3 Three-photon absorption in gallium phosphide . . . . . . . 144

C.3 Conclusion . . . . . . . . . . . . . . . . . . . . . . . . . 146

$\begin{array}{lr}\text { Samenvatting } & 149\end{array}$

$\begin{array}{ll}\text { Dankwoord } & 153\end{array}$ 



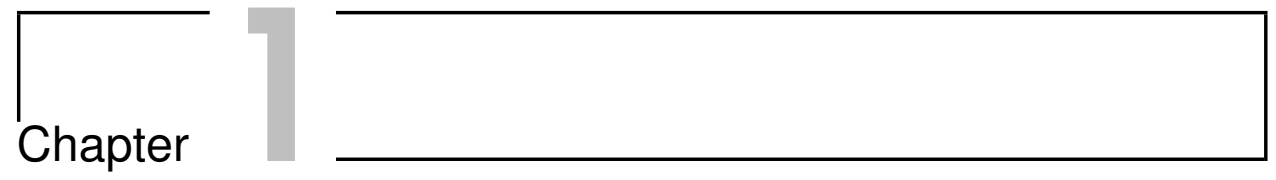

\section{Introduction}

\subsection{Photonic crystals}

\subsubsection{Intro}

There is a fast growing interest in photonic crystals to control the propagation and emission of light. Photonic crystals are composite materials whose refractive index varies periodically on length scales that match the wavelength of light [1]. The optical properties of photonic materials are determined by the spatially varying refractive index, analogous to the periodic potential for an electron in a crystal $[2,3]$. Large spatially periodic variations of the refractive index cause a strong interaction between light and the composite structure. The periodicity causes Bragg diffraction of the scattered light along a specific direction. Bragg diffraction causes stop gaps; frequency ranges for which light is not allowed to propagate along a certain direction [4,5]. A major goal of the field is the realization of three-dimensional structures that possess a photonic band gap; a frequency range in which a stop gap appears for all directions simultaneously [6, 7].

At frequencies inside the band gap, the optical density of states vanishes. This should completely inhibit spontaneous emission of sources inside the photonic crystal [6]. Strong modifications of the spontaneous emission lifetime have recently been demonstrated in photonic crystals [8-11]. In the presence of weak controlled disorder, Anderson localization of light is also predicted [7]. In this case, a photon may be trapped at a point defect which serves as a cavity with a high quality factor [12]. 


\subsubsection{Bragg diffraction in photonic crystals}

Scattering of electromagnetic waves from a periodic scattering potential causes the waves to diffract. For wavelengths of the order of the lattice spacing, constructive interference can give rise to Bragg diffraction of the scattered waves. Fig. 1.1 shows a schematic picture of Bragg diffraction of incoming waves on an atomic lattice. The constructive interference condition is met if the path difference between reflections from successive planes equals an integer times the wavelength $\lambda_{c}$ of the incoming wave:

$$
m \lambda_{c}=2 d \cos \theta,
$$

where $m$ is an integer number that indicates the order of the diffraction, $d$ is the lattice spacing and $\theta$ the angle of the incident beam with respect to the lattice plane (see Fig. 1.1). Bragg diffraction was first studied in X-ray diffraction experiments bulk materials [13].

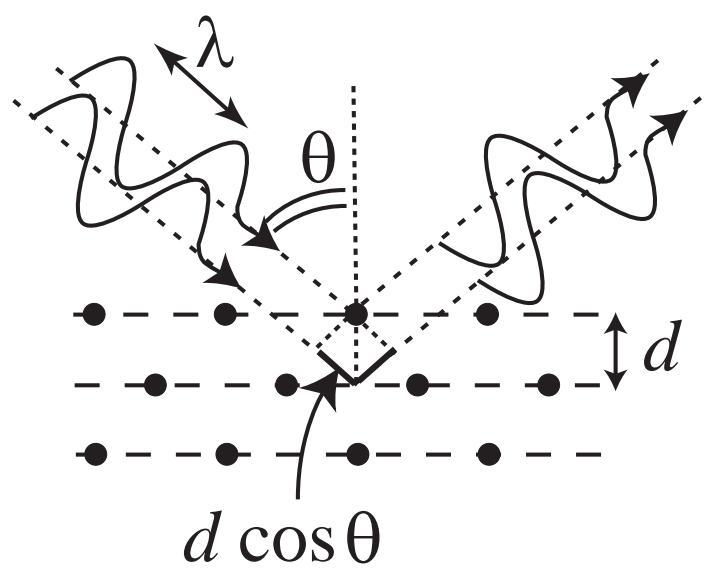

Figure 1.1: Schematic representation of Bragg diffraction. Waves are diffracted from lattice planes with distances $d$ and interfere constructively if $\lambda_{c}, d$ and $\theta$ satisfy the Bragg law, equation 1.1

In photonic crystals, the lattice parameter is commensurate with optical frequencies, causing strong Bragg diffraction to occur in this frequency range. Bragg reflection of optical waves has been studied extensively for multilayer structures such as Bragg mirrors [4]. The dispersion relation of light that travels normal to a set of lattice planes in a photonic crystal is shown schematically as a solid curve in 
Fig. 1.2. As the wave vector $k=2 \pi / \lambda$ approaches $m \pi / a$, the Bragg condition condition is met. At frequencies near the Bragg condition, the solutions of the dispersion relation split into two branches. In between the branches no solutions for propagating modes exist and therefore a stop gap appears. The center frequency of the stop gap $\omega_{c}=2 \pi c / \lambda$ is predicted by Bragg's law adjusted for photonic crystals [14]:

$$
\omega_{c}=\frac{\pi}{n_{e f f} d \cos \theta},
$$

where $n_{e f f}$ is the average refractive index of the crystal, which is obtained through e.g. the volume averaged dielectric constant $n_{e f f}=\sqrt{\bar{\epsilon}}$. For comparison, the dashed line depicts the dispersion relation $\omega c k / n_{e f f}$ for a homogeneous medium with refractive index $n_{\text {eff }}$.

At the Bragg condition, there are two counterpropagating waves in the crystal: the incident wave with wavevector $\pi / a$ and the Bragg reflected wave with wavevector $-\pi / a$ respectively. The waves form a standing wave in the crystal. Let us consider the two extreme cases of these standing waves. The first one is concentrated in the high index material, and the second one is concentrated in the low index material. Since both standing waves experience a different refractive index, they have a different optical frequency $\omega=c k / n_{e f f}$ (see Fig. 1.2). The standing wave that is predominantly located in the high index material occurs at $\omega_{\text {low }}$, at the lower edge of the stop gap. The standing wave that is mostly located in the low index material corresponds to a higher optical frequency $\omega_{\text {high }}$. As the refractive index contrast increases, the width of the stop gap $\Delta \omega / \omega_{c}$ also increases.

\subsubsection{Photonic strength}

An important parameter that gauges how strongly light interacts with any photonic nanostructure is the "photonic strength" $S$. The photonic strength is defined as the ratio of the polarizability of each structural unit cell to the physical volume. The photonic strength is a general parameter that allows to gauge and compare many different structures, including completely disordered ones or structures containing sharp atomic resonances. Experimentally, the photonic strength is gauged by the measured relative bandwidth of the first order stop band in absence of disorder broadening [15]. The stop band width increases with the refractive index contrast in the structure.

Mathematically, the photonic strength in a dielectric photonic crystal can be identified with the normalized first Fourier component of the dielectric function of the crystal: [16] 


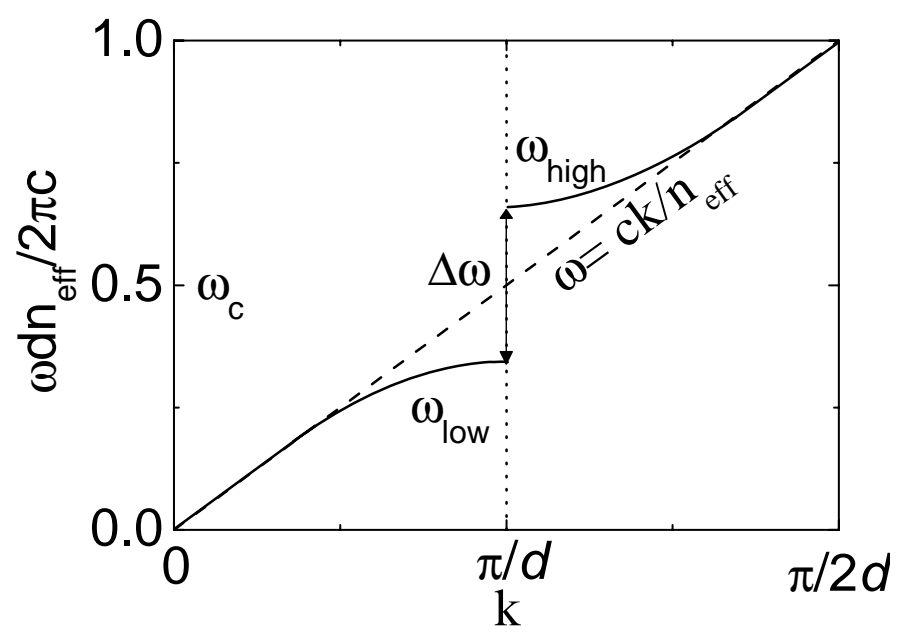

Figure 1.2: Sketch of the dispersion relation between wave vector and frequency (in units of $\left.\omega d n_{e f f} / 2 \pi c\right)$ for light propagating perpendicular to a set of lattice planes with lattice spacing $d$. A stop gap appears for frequencies $\omega_{\text {low }}<\omega<\omega_{\text {high }}$. The dashed line is the dispersion of light propagating in a homogeneous medium with refractive index $n_{\text {eff }}$.

$$
S=\frac{\Delta \omega}{\omega_{c}} \approx \frac{|\Delta \epsilon|}{\bar{\epsilon}}\left|g_{\text {Ghkl }}\right|=\frac{\left|n_{h}^{2}-n_{l}^{2}\right|}{f n_{h}^{2}+(1-f) n_{l}^{2}}\left|g_{\text {Ghkl }}\right|,
$$

where $\omega_{c}$ is the center frequency of the stop band, $\bar{\epsilon}$ is the volume averaged dielectric function, $f$ is the volume fraction of the high index material, and $\Delta \epsilon$ is the difference between the dielectric function of the high $\left(\epsilon_{2}=n_{h}^{2}\right)$ and low $\left(\epsilon_{1}=n_{l}^{2}\right)$ index materials. The Fourier coefficient $g_{\mathrm{G} h k l}$ is specific for the spatial distribution of high and low index material over the unit cell, but independent of the values $\epsilon_{h}$ and $\epsilon_{l}$. The photonic strength is inversely proportional to the Bragg length $L_{B}$ which is the 1/e decay length of light at the center frequency of the stop band,

$$
L_{B}=\frac{2 d}{\pi}
$$




\subsubsection{D photonic band gaps}

In $3 \mathrm{D}$ photonic crystals with a sufficiently large photonic strength $(S \gtrsim 0.15$ to 0.2 ), stop gaps from different sets of lattice planes in the crystal are present in all directions simultaneously, causing a 3D photonic band gap to appear. The first proposed photonic band gap structure was a face centered cubic (fcc) lattice of dielectric cubes [6]. Experimentally, the first photonic band gap materials were fabricated in the microwave range. The first structures consisted of fcc lattices of air spheres in a dielectric background [18]. However, at the high symmetry $U$ and $\mathrm{W}$ points in the Brillouin zone, a degeneracy appears, leading to a pseudo gap rather than a band gap $[19,20]$. An important result of this degeneracy is that in fcc structures, a band gap can only occur in the range of second order Bragg diffraction [21]. The minimum refractive index contrast required for a band gap to open up is 2.8 [21].

Much more relaxed conditions apply to crystals with a diamond symmetry, such as the "woodpile" photonic crystals that were proposed by Ho et al. [22]. the reason is that in diamond structures, the band gap appears in the range of first order Bragg diffraction. Moreover, the gap is less sensitive to disorder [17]. The minimum index contrast required for a band gap is reduced to 2.0.

\subsubsection{Photonic band gap crystals}

Soon after the proposal of Ho et al. [22], research was focused on woodpile photonic crystals with a diamond symmetry. Woodpile crystals are made using a layerby-layer approach. The structures were first demonstrated for microwaves and have subsequently been downscaled to near-infrared wavelengths [23-26]. The maximum thickness of structures that are fabricated by the layer-by-layer technique is limited by the lateral alignment precision of the rod layers with respect to each other, and is typically less than 8 layers or 2 unit cells.

Much larger crystals can be obtained through self assembly of colloids. Colloids have the natural ability to spontaneously form three dimensional face-centered cubic $(f c c)$ crystals [27], and are therefore suitable for the production of thick three dimensional photonic crystals. While the photonic strength of self-assembled colloidal crystals is rather low, the colloids can be made to form opals [28] that serve as a template for high-strength inverse opals, see Fig. 1.3A [29-31]

While the refractive index contrast in titania inverse opals $(2.7 \pm 0.4)$ marginally fulfills the requirement for band gap formation, the crystals were observed to show omnidirectional reflectivity features [32]. Titania inverse opals, operating at visible wavelengths were used in the first demonstration of control of spontaneous 
emission lifetimes of light sources by photonic crystals [8]. Meanwhile, much more has been done on the infiltration of high refractive index semiconductors such as Si into thin film opaline crystals [33,34]. Indeed, Si inverse opals demonstrate high reflectivity peaks whose frequency overlaps along several high-symmetry directions [34, 35]. Note that the use of semiconductors limits the range in which a photonic band gap can be obtained to frequencies below the absorption edge of the semiconductor $\left(\omega<9345 \mathrm{~cm}^{-1}\right.$ for $\left.\mathrm{Si}\right)$, which is outside the visible range.

An important issue in the vertical controlled drying method [36], which is used to fabricate the opaline templates is the lack of control over the crystal symmetry [37]. Recent results have demonstrated that some control over the lattice symmetry and orientation can be obtained through the use of patterned templates [38-41], see Fig. 1.3B. A suitable technique to fabricate the required large ordered templates is laser interference lithography [42, 43], see Fig. 1.3C. Multiple beam holography can also be used to fabricate 3D structures in photoresist without recourse to colloids [44-46]. For a band gap to appear in such crystals, the photoresist should be replaced by a semiconductor through a double inversion process which has recently been demonstrated [47].

Recently, new techniques for producing photonic crystals with diamond symmetry have been demonstrated. Direct laser writing in photoresist $[48,49]$ or in high refractive index chalcogenide glasses [50] allows for the fabrication of defects inside three dimensional photonic crystals. Furthermore, three dimensional photonic crystals were fabricated by a two-step etching process [51,52] or by focused ion beam milling [53], which allows the fabrication of crystals with a diamond symmetry single crystalline wafers, see Fig. 1.3D.

\subsection{Switching photonic crystals}

\subsubsection{Density of states switching}

In the majority of photonic crystal studies, the photonic crystals themselves are stationary. To explore active functionalities for photonic crystals, it is vital to investigate whether ultrafast switching of photonic stop bands and even of band gaps is feasible. Changing the refractive index of the structure offers ultrafast control over the photonic strength.

Switching 3D photonic crystals is particularly interesting, as it provides dynamic control over the optical density of states inside the crystal [54]. Ultrafast control of the density of states has exciting prospects: First of all it would allow dynamic control over spontaneous emission of light sources; sources whose fre- 

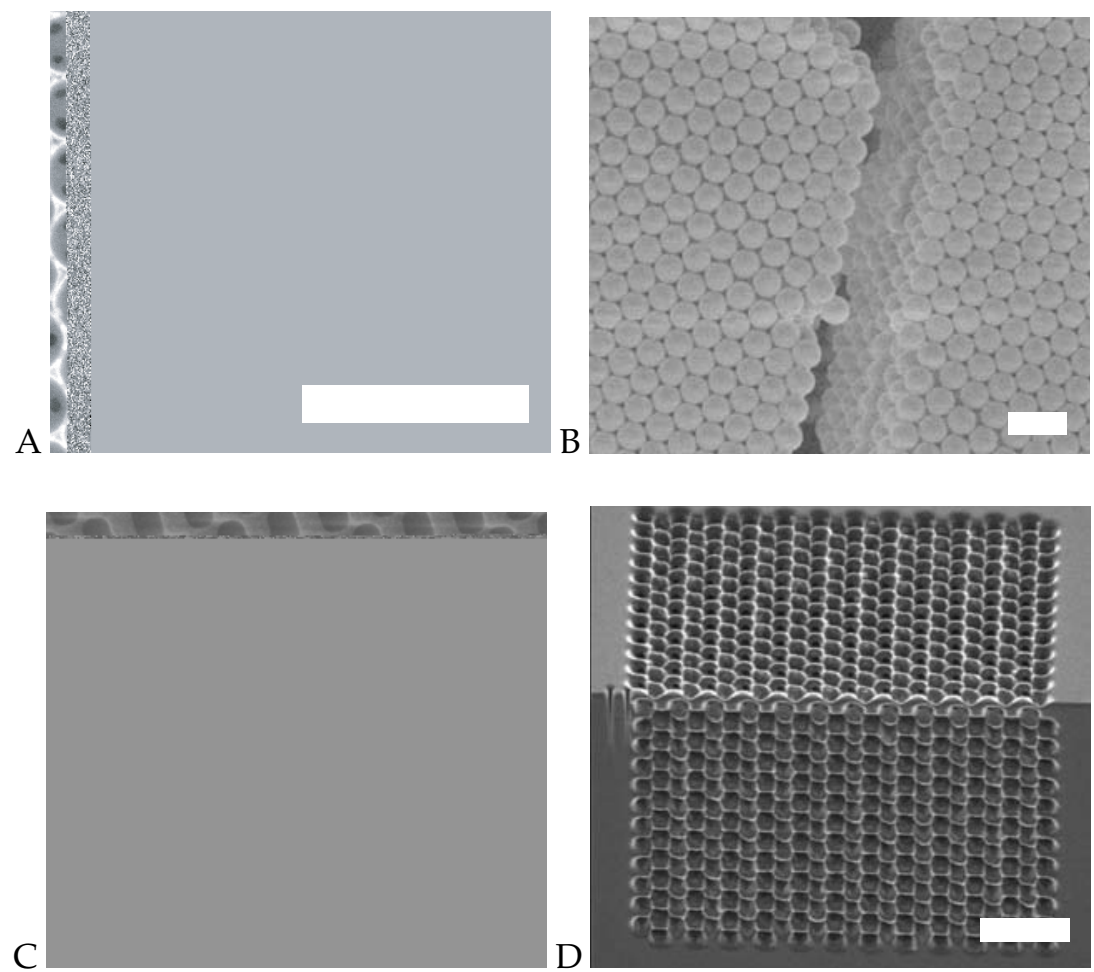

Figure 1.3: SEM images of various photonic crystals. A $\{111\}$ plane of air holes in a titania inverse opal. (B) $\{111\}$ plane of a fcc close packed crystal of polystyrene spheres, created by vertical controlled drying method on a patterned substrate [40]. (C) hexagonal array of holes in photoresist, fabricated by laser interference lithography and used to control the deposition of colloidal spheres [40]. (D) focussed ion beam etching in gallium phosphide. Scalebars represent $2 \mu \mathrm{m}$. Images courtesy of Lydia Bechger (A) and Willem Tjerkstra (D).

quency is in a range where the DOS is strongly reduced, could suddenly experience an increased DOS, resulting in an accelerated spontaneous decay. Vice versa, if the DOS is quickly switched from high to low, a light source will suddenly be shut off. To the best of our knowledge these remarkable properties are unique for light in photonic crystals, since no analogy exists for electrons in semiconductors.

Spontaneous emission of light sources that are embedded into a cavity, is enhanced by the Purcell effect [55]. Under certain conditions, light sources can become strongly coupled to a single cavity mode. Strong coupling of quantum dot light sources to narrow cavity modes was recently demonstrated in photonic nanocavities [56-58]. In such coupled systems, switching the cavity resonance frequency 
would allow dynamic control over the coupling strength between cavity and a single emitter.

\subsubsection{Propagation switching}

The directional propagation of light inside photonic crystals strongly deviates from bulk dielectric materials. Near the stop band edges, the angle of propagation strongly depends on frequency, leading to so-called superprism effects [59]. Also the propagation of diffusive light inside photonic crystals is strongly influenced by stop bands, resulting in non-Lambertian behavior [60]. Switching of photonic crystals could dynamically control the direction in which light is diffracted, offering a new mechanism for all optical beam steering.

In photonic crystal waveguides, light can be confined to a single mode. The group velocity of pulses propagating in such waveguide is characterized by the slope of the dispersion relation $\delta \omega / \delta k$. Recently, very low group velocities have been observed in photonic crystal waveguides near flat parts of the dispersion relation [61, 62]. We propose that ultrafast switching switching mechanisms can be used to shift the dispersion curve up or down on a ps timescale. Consequently, a pulse with frequency $\omega$ could suddenly experience a different group velocity. Switching could in this way serve as a mechanism to slow-down or accelerate light pulses. An interesting prospect is that if the crystal is adjusted in an adiabatic way, it will drastically enhance the coupling efficiency of light pulses into very slow waveguides modes. Similar dynamic control over the coupling efficiency into a cavity, allows for adiabatic and reversible compression of a pulse into a single narrow cavity mode, as was demonstrated in the proof-of-principle experiment of Ref. [63]. A scheme involving multiple coupled cavities to slow down light coherently was proposed by Ref. [64].

In absence of photonic crystals, ultrafast switching of bulk semiconductors finds applications in high speed optical modulators [65] and multilayer stacks [66, 67]. Last but not least, ultrafast control of photonic crystals is important for controlling the propagation of light, such as in switched macroporous silicon [68], or 2D crystal slabs [69].

\subsubsection{Relevant timescales}

The analysis of the changes in the density of states in switched photonic crystals by Ref. [54] has been quasi static. The photonic crystal is assumed to adjust to the new situation adiabatically. The adiabatic theorem states that a physical 
system remains in its instantaneous eigenstate if a given perturbation is acting on it slowly enough and if there is a gap between the eigenvalue and the rest of the Hamiltonian's spectrum [70]. In the case of a switched photonic crystal, the eigenstates are the Bloch modes of the crystal. During the switching, the Bloch modes are changed by a time dependent refractive index, which changes the scattering potential, and can thus be considered the perturbation. It is interesting to investigate what the timescale of the switching should be for the change to become non-adiabatic. Ref [54] proposed that switching on time scales faster than the inverse width of the band gap $(1 / \Delta f)$ would result in non-adiabatic effects. In their analysis this would correspond to a switching time of $160 \mathrm{fs}$.

It is also interesting to consider photons in a Bloch mode whose frequency is located at the upper edge of the band gap of the unswitched crystal. During the switching, which shifts the band gap up in frequency, these photons will forced to change their color. In this case the inverse distance from the Bloch mode to the edge of the gap has been suggested as a relevant time scale [71]. The energy required for the frequency conversion can be gained from the pump field. The same situation applies when we consider the switching of a single mode cavity, whose resonance frequency is shifted.

An analogy of transitions between transitions between Bloch states and electronic interband transitions has been made by Ref. [72]. Their analysis shows that photons can indeed transfer into from a Bloch mode at frequency $\omega_{1}$ into a mode at frequency $\omega_{2}$ if the refractive index is periodically modulated. The modulation frequency, however, must of the order of the frequency difference $\omega_{2}-\omega_{1}$ of the modes modes. A $\chi^{(2)}$ nonlinearity in GaAs is proposed to induce such fast periodic oscillations [72]. Transition times of the order of $100 \mathrm{ps}$ are predicted in an experiment where $\omega_{2} \approx 2 \omega_{1}$.

Even though several interesting switching experiments on photonic crystals have been demonstrated, there are still many open elementary questions. In particular, how the optical properties of a photonic crystal behave at frequencies in the vicinity of a photonic band gap that is switched on ultrafast time scales. As a first step we will study in this Thesis the ultrafast reflectivity changes of such crystals. Our results will provide useful insights which can be used in severely challenging experiments in which ultrafast changes of the density of states are observed. 



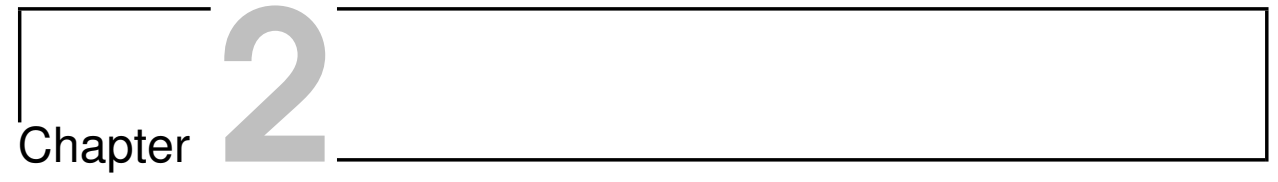

\section{Experimental considerations}

\subsection{Experimental requirements}

In optical switching experiments, four important requirements have to be met [54]. First of all, the magnitude of the induced change in the real part of the refractive index $n^{\prime}$ must be large enough to obtain the desired effect. A relative change in $n^{\prime}$ of $5 \%$ is required to switch a Si inverse opal by its band gap width, resulting in a major change in the density of states. Such large changes can be induced by free-carrier generation [73] but are not achievable with Kerr switching.

The second requirement is the minimum time scale $\Delta$ t over which the switching process is turned on. In experiments where light pulses are trapped inside photonic crystals, or where a non-adiabatic response is studied, a switching time scale on the order of a few hundred femtoseconds is necessary. The time scale for switching off is of less fundamental interest but more so for potential applications, since this timescale determined how fast a phenomenon could be repeated.

The third requirement is that the absorption of probe light, gauged by $n^{\prime \prime}$, should be small in the switched sample. Induced absorption becomes particularly important inside photonic crystal resonators where absorption-induced cavity losses can limit the Q-factor. The fourth requirement concerns the spatial homogeneity of the change $\Delta n^{\prime}$ in a sample. Homogeneity is particularly important in switching the density of states in $3 \mathrm{D}$ photonic crystals. A large gradient in $\Delta n^{\prime}$ in the crystal results in a highly chirped switched sample, which can no longer be considered a photonic crystal.

All of these requirements also pertain to other applications of switched semi- 
conductors, such as in waveguiding $[65,74]$ albeit much relaxed. Therefore, we expect the results from the present study also to be relevant for applications outside photonic crystals.

\subsection{Switching mechanisms}

\subsubsection{Introduction}

Dynamical control over the optical properties of photonic crystal can be obtained by controlling either the refractive index $n^{\prime}+i n^{\prime \prime}$ of the backbone material, or the lattice spacing as a function of time.

Control over the lattice spacing has been demonstrated in weakly photonic crystals by heating of a colloidal crystal that was embedded in a polymer gel [75] and by mechanical compression of polymer photonic crystals [76]. While large changes in directional Bragg diffraction have been demonstrated, the refractive index contrast in elastic photonic materials is too small for a band gap to appears. In high refractive index semiconductor photonic crystals, compressive sound waves may be utilized to induce small changes in the lattice constant. The maximum speed of such variations, however, is limited to the $\mathrm{GHz}$ range due to ultrasound absorption.

An elegant method to tune the resonance frequency of photonic crystal cavities that was proposed by Ref. [77] is to introduce small dielectric perturbations in the near field of a photonic crystal cavity. Experimental demonstrations of such nano-mechanical tuning were recently given by Refs. [78, 79].

Photonic crystals can also be tuned by liquid crystal infiltration, as was proposed theoretically by [80, 81]. The ordering and alignment of birefringent materials such as liquid crystals can be controlled by temperature $[82,83]$ or electric fields [8486]. With liquid crystals, large refractive index variations up to $10 \%$ are feasible. Unfortunately, the tuning process is inherently slow; typical time scales in the millisecond to microsecond range.

Large refractive index changes of the backbone can also be attained by heat induced phase transitions in opal $\mathrm{VO}_{2}$ composites [87]. Ultrafast changes in the Bragg peak of such structures with switching times of 350 fs have recently been demonstrated [88]. The recovery to the unswitched phase is typically slower, of the order of $100 \mu \mathrm{s}$ [89]. Furthermore, since the switching process yields metallic states, absorption in the switched structure is also an important issue.

Non-linear optical techniques incorporating the optical Kerr effect can produce instantaneous changes in the index of refraction as well as ultrafast recovery at time 
scales governed by the duration of the pump pulse. We estimate, however, that the induced Kerr changes are typically an order of magnitude smaller than that achieved via free-carrier absorption. Nevertheless, optical Kerr switching proves to be complementary to free-carrier excitation for ultrafast switching studies.

\subsubsection{Free carrier excitation}

Several interesting switching experiments have recently been carried out in which a free-carrier plasma was excites in the backbone of photonic structures. A first switching experiment on a thin Bragg mirror of 5 pairs of layers was carried out by Ref. [66]. Unfortunately, the switched transmission was measured at one probe frequency only, leaving little room for physical interpretation. Pioneering switching experiments were carried out by Leonard et al. in 2D Si photonic crystals [68]. The carrier density generated in their experiment was sufficient to induce a clear shift of the blue edge of the Bragg stop gap on ultrafast time scales. The experiments revealed an important limitation: the linear absorption of the pump beam leads to a spatial inhomogeneity in the degree of switching. Leonard et al. deduced that only a layer of three unit cells near the sample surface was switched [68]. Switching experiments on a thick GaAs-AlAs Bragg mirror were carried out by Ref. [67]. Unfortunately, the pump frequency was chosen in the linear absorption regime of GaAs, limiting the switching homogeneity length $\ell_{\text {hom }}$ to only 1.1 pairs. The measured absolute change in the transmission $\Delta \mathrm{T}$ was less than $6.3 \times 10^{-3} \%$. Similar inhomogeneity may also have played a role in interesting studies of silicon infiltrated opaline crystals, where reflectivity changes were observed near a Bragg peak at frequencies above the Si-band gap [90].

Switching 3D crystals such as inverse opals potentially offers the largest effect on the density of states, especially near the band gap in the range of the second order stop bands [54]. Experiments in the first order stop gap region were carried out by Refs. [90] and [91], and switching experiments in the second order stop band region by Ref. [92]. Unfortunately, the analysis of reflectivity and transmission spectra is quite complicated in the second order region, where multiple Bragg wave coupling must be taken into account to interpret the propagation of light through such structures [32]. Simple two band models such as proposed by Refs [90] and [91] neglect multiple Bragg wave coupling, and are thus not quite appropriate for 3D photonic band gap crystals. Switching experiments on woodpile photonic band gap crystals with where the band gap is expected in the range of first order Bragg diffraction were recently demonstrated by us [93]. 


\subsubsection{Optimal conditions}

In our switching experiments, an ultrashort laser pulse optically generates a freecarrier plasma with a density $N_{e h}$. The dielectric response of semiconductors in which free carriers are excited is well described by the Drude model $[3,94]$. Under the assumptions given in Appendix A, the complex refractive index of semiconductors in which a free-carrier plasma is generated is approximated by:

$$
n=n^{\prime}+i n^{\prime \prime}=\sqrt{\epsilon(\omega)} \simeq \sqrt{\epsilon_{B G}(\omega)-\left(\frac{\omega_{p}}{\omega}\right)^{2}\left(1-\frac{i}{\omega \tau_{D}}\right)},
$$

where $\omega$ is the frequency of the probe light, $\epsilon_{B G}$ the dielectric constant of the bulk material, $\tau_{D}$ the momentum relaxation time of the carriers, and $\omega_{p}$ the plasma frequency. The plasma frequency is proportional to the generated carrier density, and is defined as $\omega_{p} \equiv N_{e h} e^{2} / \epsilon_{0} m_{o p t}^{*}$, where $m_{o p t}^{*}$ is the optical effective mass of the carriers.
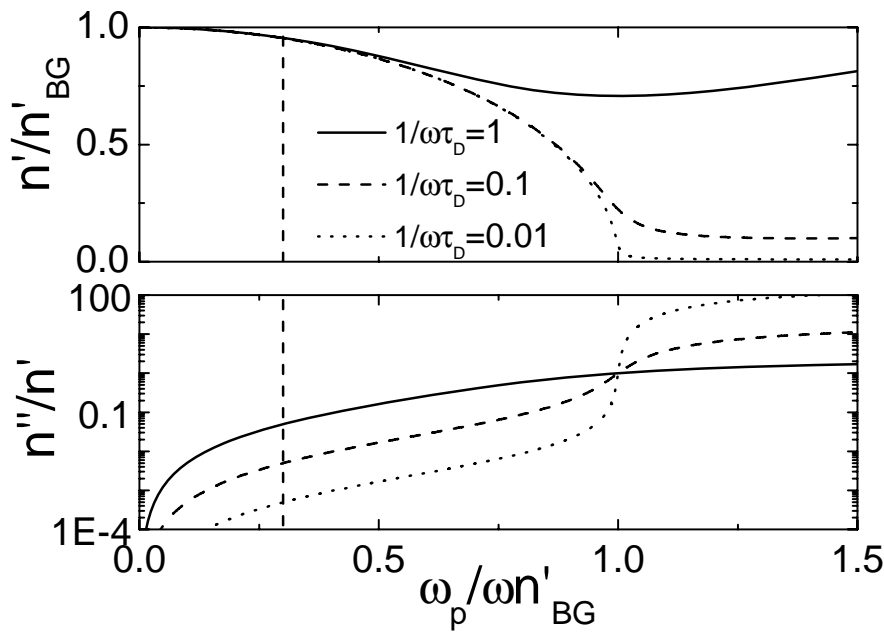

Figure 2.1: Real part of the refractive index $n^{\prime}$, normalized to $n_{B G}^{\prime}$ (top) and attenuation index $n^{\prime \prime} / n^{\prime}$ (bottom) of a semiconductor after excitation of a free-carrier plasma. Data is plotted as a function of normalized plasma frequency $\omega_{p} / \omega n_{B G}^{\prime}$. The curves correspond to damping parameters $1 / \omega \tau_{D}=1$ (solid) $1 / \omega \tau_{D}=0.1$ (dashed), and $1 / \omega \tau_{D}=0.01$ (dotted). The vertical dashed line indicates optimum pumping condition for poly-Si.

Fig. 2.1 shows the Drude response of a semiconductor in which free carriers have been generated. The upper panel shows the real part of the refractive index, normalized to the real part of the bulk refractive index $n_{B G}=\sqrt{\epsilon_{B G}}$, as a function of 
the scaled plasma frequency $\omega_{p} / \sqrt{\epsilon_{B G}} \omega$. The lower panel shows the corresponding imaginary part of the refractive index, relative to the real part $n^{\prime \prime} / n^{\prime}$. Curves are plotted for three different damping parameters.

At the critical carrier density $\omega_{p}^{*} \equiv n_{B G} \omega$, we observe that all curves tend to the abscissa. At the critical density, $\epsilon$ is purely imaginary, and thus the imaginary part of the refractive index is equal to the real part (see lower panel), irrespective of the damping parameter. Experimentally, this corresponds to a situation where probe light is absorbed within a single optical cycle.

The induced absorption can be strongly reduced by choosing a plasma frequency below the $\omega_{p}^{*}$. Here we choose a probe wavelength $\lambda=1.5 \mu \mathrm{m}$. We take a damping parameter $1 / \omega \tau_{D}=0.1$, using a relaxation time $\tau_{D}=10 \mathrm{fs}$, which is typical for the poly-Si backbone material of photonic crystals discussed in Chapter 6 and 7. Optimum switching conditions are found at a plasma frequency that is about $\omega_{p}=0.3 \omega_{p}^{*}$, indicated by the vertical dashed line in Fig. 2.1. The change in real part of the refractive index at this frequency is about $-5 \%\left(n^{\prime} / n_{B G}^{\prime} \approx 0.95\right)$, while the induced absorption remains limited to $n^{\prime \prime} / n^{\prime}=0.005$. In single crystalline materials, the damping parameter is typically ten times smaller: $1 / \omega \tau_{D}=0.01$ [73], and plasma frequencies up to $\omega_{p}=0.7 \omega_{p}^{*}$ are feasible. The corresponding refractive index change would increase to $-30 \%\left(n^{\prime} / n_{B G}^{\prime} \approx 0.70\right)$, while the induced absorption remains unchanged $\left(n^{\prime \prime} / n^{\prime}=0.005\right)$.

In summary, we find good experimental conditions for free-carrier plasma frequencies around $\omega_{p}=0.3 n_{B G} \omega$. Large changes in refractive index are feasible, while the induced absorption remains limited. 



\section{Spatial homogeneity of switched photonic crystals and of bulk semiconductors}

We investigate the spatial homogeneity of optically generated free-carrier plasmas in semiconductors. We discuss a nonlinear absorption model that takes into account both linear and two-photon absorption processes. From this model, we define a homogeneity length scale to quantify homogeneous switching conditions and we derive optimum pumping conditions for Si and GaAs. In particular, we trace constant homogeneity lengths in a general parameter-diagram that pertains to any switchable material, including all semiconductors. We discuss the role of disorder-induced diffusion of the pump beam in photonic crystals.

\subsection{Introduction}

In homogeneous optical switching experiments, several important length scales should be taken into account. We consider the homogeneity of the pump light by introducing homogeneity length $\ell_{\text {hom }}$ that gauges the size of the homogeneously changed volume inside the sample. The following criterium in the stop band holds for any switching experiment:

$$
\ell_{\text {hom }}>\ell_{\text {probe }}
$$

In photonic crystals, at probe frequencies in the stop band, the penetration dept of 
probe light is limited by the Bragg length $\left(\ell_{\text {probe }}=L_{B}\right)$. The homogeneity length should therefore exceed the Bragg length:

$$
\ell_{\text {hom }}>L_{B}
$$

to avoid chirped switched samples whose reflectivity properties are difficult to interpret [68]. An optimum effect of switching on the photonic properties of a crystal is therefore only obtained if the homogeneity length exceeds the Bragg length $\ell_{\text {hom }}>L_{B}$.

Here, we discuss the homogeneity of optically switched semiconductors and switched photonic crystals. In our analysis, we concentrate on silicon and GaAs. Examples are given for light in the telecom band at $\omega_{\text {tele }} / \mathrm{c}=6450 \mathrm{~cm}^{-1}(\lambda=1550 \mathrm{~nm})$, but can easily be generalized to other frequencies. We set a minimum volume of 5 unit cells cubed in which the change $\Delta n^{\prime}$ must remain within $10 \%$ of its maximum value (see Chapter 2 ). This homogeneity requirement holds for both the lateral directions $x$ and $y$, as well as for the z-direction defined in Fig. 3.1. Even in such a small crystal volume, the local density of states already shows a significant decrease for frequencies that lie in the band gap [95]. The typical unit cell size of silicon inverse opal photonic crystals with a band gap near $\omega_{\text {tele }}$ is $a=1.2 \mu \mathrm{m}$, therefore the homogeneously switched area must extend at least $6 \mu \mathrm{m}$ in all three dimensions.

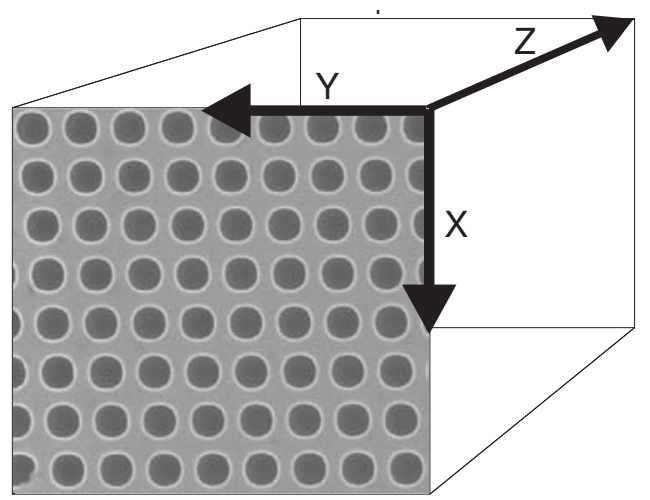

Figure 3.1: Schematic image of a sample: The z-axis is defined to be pointing into the sample, the $x$ and $y$-directions are the lateral directions. In the figure, the front face of the sample displays a SEM image of a dry etched Si 2D photonic crystal with a slightly paralellogrammic latice (lattice angle 85 deg). The lattice parameter a is equal to $750 \mathrm{~nm}$. SEM courtesy of L.Woldering. 


\subsection{Optical properties of silicon}

To analyze the switching homogeneity in the z-direction, we briefly consider how pump light is absorbed in semiconductors. At low pump irradiances $I$, the absorption of light in semiconductors is proportional to the irradiance: $\alpha I$. The absorption coefficient $\alpha$ tends to zero for photon energies $\hbar \omega_{\text {pump }}$ below the electronic band gap energy $\mathrm{E}_{\text {gap }}$, see Fig. 3.2 for silicon. With increasing pumping irradiance, nonlinear two-photon absorption starts to play an important role. For two-photon absorption, the absorption is proportional to $\beta I^{2}$ where $\beta$ is the two-photon absorption coefficient. This coefficient is expected to vanish for $\hbar \omega_{\text {pump }}>\mathrm{E}_{\text {gap }} / 2$. Fig. 3.2 displays the frequency dependence of the linear and two-photon absorption coefficients of silicon. The data were obtained from Refs. [96-99]. We have performed z-scan measurements [100] to obtain the two-photon absorption coefficient $\beta$ at two additional wavelengths (see Appendix C). For bulk silicon at $\omega_{\text {pump }}=5000 \mathrm{~cm}^{-1}, \beta$ was measured to be $0.20 \pm 0.05 \mathrm{cmGW}^{-1}$. At $\omega_{\text {pump }}=6250 \mathrm{~cm}^{-1}$, we obtained $\beta=0.80 \pm 0.1$ $\mathrm{cmGW}^{-1}$, in good agreement with the value $\beta=0.88 \pm 0.13 \mathrm{cmGW}^{-1}$ at $\omega_{\text {pump }}=6494$ $\mathrm{cm}^{-1}$ from Ref. [97]. Our measurements confirm that $\beta$ tends to zero for photon energies approaching $\mathrm{E}_{\text {gap }} / 2$. Both the linear and the nonlinear absorption coefficient can thus be controlled by varying the pump frequency.

\subsection{Homogeneity of switched semiconductors}

\subsubsection{Theoretical model}

We now present a model for the carrier density depth profile $\mathrm{N}_{e h}(\mathrm{z})$ caused by absorption of pump light. The absorbed irradiance is described by the nonlinear differential equation

$$
\frac{d I(z)}{d z}=-\left[\alpha I(z)+\beta I^{2}(z)\right],
$$

which we have solved by implicit integration [101]. The resulting expression describing the irradiance depth profile is

$$
I(z)=\frac{I_{0} e^{-\alpha z}}{1+\left(\beta I_{0} / \alpha\right)\left(1-e^{-\alpha z}\right)^{\prime}},
$$

where $I_{0}$ is the irradiance at the interface. The resulting carrier density profile $\mathrm{N}_{e h}(\mathrm{z})$ is related to the irradiance depth profile $I(z)$ as

$$
N_{\text {eh }}(z)=\frac{I(z) \tau_{\text {pump }}}{\hbar \omega_{\text {pump }}}\left[\alpha+\frac{1}{2} \beta I(z)\right],
$$




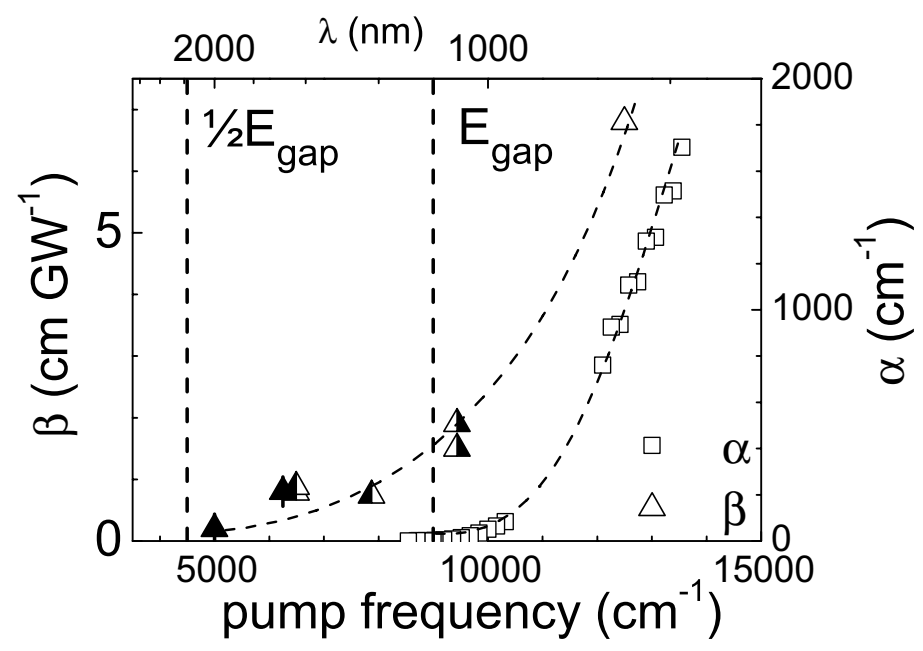

Figure 3.2: Absorption coefficients $\alpha$ and $\beta$ of Si versus pump frequency. The dashed vertical lines correspond to photon energies of $E_{\text {gap }} / 2$ and $E_{\text {gap }}$. The open squares indicate linear absorption coefficients taken from Ref. [96] (right-hand scale). The solid triangles indicate the two-photon absorption coefficients that we have determined by z-scan measurements (left-hand scale). The leftfilled triangles indicate the values from Ref. [97], the right-filled triangles are data from Ref. [98], and the open triangle data from Ref. [99]

where, for practical purposes, we have assumed a square intensity envelope ${ }^{1}$ of the pump pulse with width $\tau_{\text {pump }}$ and $\hbar \omega_{\text {pump }}$ is the energy of the pump photons. The factor $1 / 2$ for two-photon absorption indicates that two photons must be absorbed to generate one electron-hole pair. Substitution of Eq. 3.4 into Eq. 3.5 allows us to calculate the carrier density profile for any given combination of $\alpha, \beta$ and $I_{0}$.

We now consider the situation where a carrier density $N_{e h}(0)=N_{0}$ is excited at the sample interface. To investigate the relation between the absorption coefficients and pumping homogeneity of the carrier density profile, we solve Eq. 3.4, and find the required pump irradiance to generate a carrier density $N_{0}$ to be

$$
I_{0}=-\frac{\tau_{\text {pump }} \alpha-\sqrt{\tau_{\text {pump }}^{2} \alpha^{2}+2 \tau_{\text {pump }} \beta N_{0} \hbar \omega_{\text {pump }}}}{\tau_{\text {pump }} \beta} .
$$

Since the maximum gradient in carrier profile appears at the interface, we consider

\footnotetext{
${ }^{1}$ The maximum homogeneity length for a Gaussian intensity envelope was found to be within $7 \%$ of the result for square pulses shown here.
} 
the z-derivative of the carrier density profile in Eq. 3.5, and use Eq. 3.3 to obtain

$$
\frac{1}{N_{0}}\left(\frac{d N_{e h}}{d z}\right)_{z=0}=\frac{\left(\alpha+\beta I_{0}\right)\left(-\alpha I_{0}-\beta I_{0}^{2}\right)}{\alpha I_{0}+\frac{1}{2} \beta I_{0}^{2}} .
$$

We insert the irradiance found in Eq. 3.6 into Eq. 3.7 and find for the slope of the carrier profile:

$$
\frac{1}{N_{0}}\left(\frac{d N_{\text {eh }}}{d z}\right)_{z=0}=-\frac{2\left(\tau_{\text {pump }} \alpha^{2}+2 \beta N_{0} \hbar \omega_{\text {pump }}\right)}{\tau_{\text {pump }} \alpha+\sqrt{\tau_{\text {pump }}\left(\tau_{\text {pump }} \alpha^{2}+2 \beta N_{0} \hbar \omega_{\text {pump }}\right)}} .
$$

In order to quantify the homogeneity of a switched sample, we define the homogeneity length $\ell_{\text {hom }}$ within which the carrier density remains within $10 \%$ of its surface value:

$$
\ell_{\text {hom }} \equiv-0.1 \times\left[\frac{1}{N_{0}}\left(\frac{d N_{e h}(z)}{d z}\right)_{z=0}\right]^{-1}=0.1 \times \frac{\tau_{\text {pump }} \alpha+\sqrt{\tau_{\text {pump }}\left(\tau_{\text {pump }} \alpha^{2}+2 \beta N_{0} \hbar \omega_{\text {pump }}\right)}}{2\left(\tau_{\text {pump }} \alpha^{2}+2 \beta N_{0} \hbar \omega_{\text {pump }}\right)} .
$$

Because the homogeneity length is directly related to the maximum gradient in the carrier depth profile, it is a helpful parameter in quantifying the homogeneity of switched semiconductors. Since for most applications the switching homogeneity is important, the homogeneity length should be much larger than the size of the switched sample $\ell_{\text {hom }}>L$. To illustrate the homogeneity length, three carrier density depth profiles are shown in Fig. 3.3 for which $\beta$ is chosen to be $2 \mathrm{cmGW}^{-1}$, and $\alpha$ is varied from zero (dotted curve) to $400 \mathrm{~cm}^{-1}$ (dashed curve) to $800 \mathrm{~cm}^{-1}$ (solid curve). For each case, $I_{0}$ is chosen such that the carrier density reaches $0.9 \times 10^{20} \mathrm{~cm}^{-3}$ at the sample interface, corresponding to a $5 \%$ change in $n^{\prime}$ at $\omega_{\text {tele }}$ in silicon. On the right y-axis the corresponding real part of the refractive index $n^{\prime}$ for silicon at $\omega_{\text {tele }}$ is shown. The pump frequency was assumed to be $5000 \mathrm{~cm}^{-1}$. A closer look at the three depth profiles in Fig. 3.3 shows that for $\alpha=0 \mathrm{~cm}^{-1}$, the homogeneity length is $1.0 \mu \mathrm{m}$. As $\alpha$ increases to $400 \mathrm{~cm}^{-1}$, the homogeneity length increases to $1.2 \mu \mathrm{m}$. As $\alpha$ increase further to $800 \mathrm{~cm}^{-1}$, the homogeneity length decreases again to $1.0 \mu \mathrm{m}$. The surprising occurrence of a maximum in the homogeneity length can be explained with the aid of Eq. 3.5. For small $\alpha\left(\alpha \ll I_{0} \beta\right)$, the absorption is dominated by two-photon absorption. If $\alpha$ increases, the pump irradiance $I_{0}$ needed to obtain the surface carrier density decreases, reducing the slope of the irradiance profile at the interface, determined by the exponent $-\left(\alpha+I_{0} \beta\right)$. This leads to an increase in $\ell_{\text {hom }}$. If $\alpha$ increases further to the regime where the absorption is dominated by linear absorption $\left(\alpha \gg I_{0} \beta\right)$, any further increase in 
$\alpha$ will result in a decrease of the homogeneity length. In the region between the two extremes, the homogeneity length thus attains a maximum value. This means that simply choosing two-photon (or perhaps even higher-photon) absorption over linear absorption is not always sufficient to ensure an optimal homogeneity.

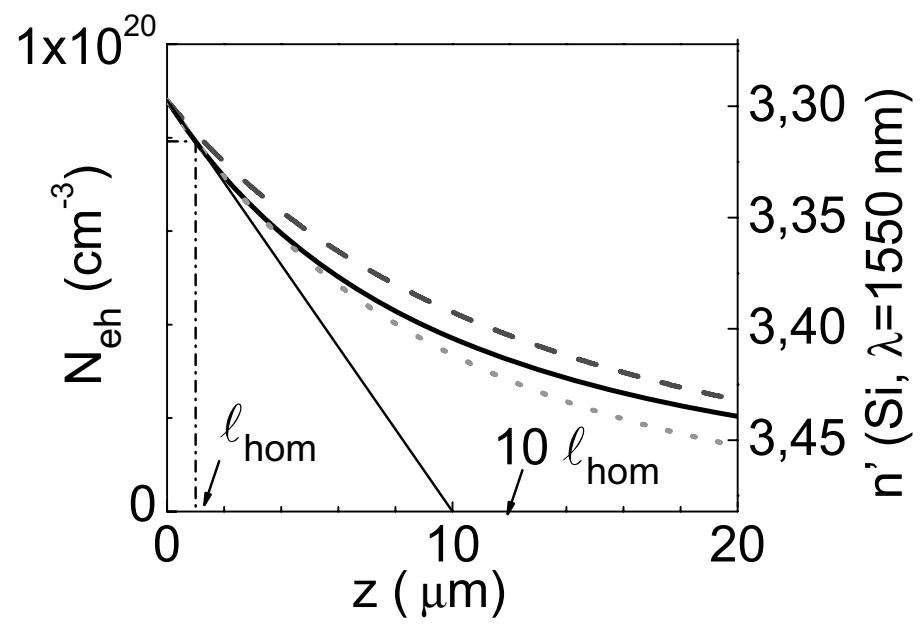

Figure 3.3: Calculated carrier density depth profile $N_{\text {eh }}(z)$ for constant $\beta=2 \mathrm{cmGW}^{-1}$ and three different linear absorption coefficients: $\alpha=0$ (solid curve), $\alpha=400 \mathrm{~cm}^{-1}$ (dashed curve) and $\alpha=800$ $\mathrm{cm}^{-1}$ (dotted curve). With $\mathrm{N}_{\text {eh }}$ at the interface kept constant at $0.9 \times 10^{20} \mathrm{~cm}^{-3}$, the necessary pump irradiance $I_{0}$ is calculated, assuming that $\tau_{\text {pump }}=120 \mathrm{fs}$ and $r_{\text {pump }}=75 \mu \mathrm{m}$. The corresponding carrier density depth profile was obtained with Eq. 3.4. The homogeneity length $\ell_{\text {hom }}$ is shown for $\alpha=0$. The right-hand scale shows the resulting refractive index $n^{\prime}$ for $\omega=6450 \mathrm{~cm}^{-1}$ light in Si.

\subsubsection{Homogeneity plot}

We propose a homogeneity plot, which is a general parameter diagram in $(\alpha, \beta)$ space, to obtain further insight in the homogeneity. First we choose a fixed electron density at the interface, for instance $N_{0}=0.9 \times 10^{20} \mathrm{~cm}^{-3}$. The corresponding constant homogeneity length contours are then deduced from our absorption model, and visualized in a plane spanned by linear and two-photon absorption coefficients, see Fig. 3.4. The three depth profiles shown in Fig. 3.3 correspond to positions $a, b$, and $\mathrm{c}$ in Fig. 3.4. It is seen that point $\mathrm{b}$ lies furthest below the $\ell_{\text {hom }}=1 \mu \mathrm{m}$ contour, as expected from since this condition has the largest homogeneity length. To obtain a 
certain minimum homogeneity, the absorption coefficients must remain below the curve corresponding to the required homogeneity length. An analytical expression for the curves was obtained by solving Eq. 3.9 for a fixed value of $\ell_{\text {hom }}$ :

$$
\beta(\alpha)=\frac{1+4 \ell_{\text {hom }} \alpha-8 \ell_{\text {hom }}^{2} \alpha^{2}+\tau_{\text {pump }} \sqrt{1+8 \ell_{\text {hom }} \alpha}}{16 \ell_{\text {hom }}^{2} \hbar \omega_{\text {pump }} N_{0}} .
$$

From Fig. 3.4, we also obtain the homogeneity length that can be obtained for a semiconductor at a certain pump frequency. The absorption coefficients for silicon at various frequencies taken from Fig. 3.2 are plotted in the plane in Fig. 3.4. The homogeneity plot therefore demonstrates how pumping homogeneity can be maximized by choosing the appropriate pump frequency. Generally, smaller absorption coefficients lead to an increased homogeneity. This increase comes at the price of a higher necessary pump irradiance $I_{0}$. From Fig. 3.4 we conclude that the most homogeneous switch for silicon can be achieved for $\omega_{\text {pump }}=5000 \mathrm{~cm}^{-1}$ where the Si-data intersects the abscissa. For this pump frequency, the homogeneity length is $3.0 \mu \mathrm{m}$. For comparison: if $\omega_{\text {pump }}$ is equal to $12500 \mathrm{~cm}^{-1}$ or $\lambda=800 \mathrm{~nm}$, the standard wavelength of Titanium Sapphire lasers that are frequently used $[67,68,90]$, the homogeneity length is only $0.6 \mu \mathrm{m}$. The necessary pump irradiance remains below the maximum available pump energy of our laser system $I_{m a x}$. Our generalized homogeneity plot is valid for all materials and allows us to find optimum switching conditions.

We have also constructed a homogeneity plot for GaAs, see Figs. 3.5A and B. The optical effective mass of free carriers in GaAs $m_{o p t}^{*}=0.058$ is 2.6 times smaller than for Si $[102,103]$. Therefore, the carrier density required for a $5 \%$ change in the refractive index in GaAs is 2.6 times lower than in silicon: $N_{0}=3.5 \times 10^{19}$ $\mathrm{cm}^{-3}$. From the resulting plots in Fig. 3.5A and B we find that the homogeneity requirement on the two-photon absorption coefficient $\beta$ in GaAs is somewhat relaxed; homogeneous switching can be achieved at larger values for $\beta$ than in $\mathrm{Si}$. However, the two-photon absorption coefficients for GaAs are much larger than in $\mathrm{Si}$, limiting the range of pump frequencies that can be used. In Fig. 3.5, the filled triangles are absorption coefficients for GaAs from Refs [97, 102]. The three lowermost data points were obtained by us through $\mathrm{z}$-scan experiments at $\lambda=1.63$ $\mu \mathrm{m}$ (open squares) and $\lambda=1.72 \mu \mathrm{m}$ (filled square) respectively (see Appendix C). At pump frequencies above the electronic band gap at $\omega_{\text {pump }}=12500 \mathrm{~cm}^{-1}$, both $\alpha$ and $\beta$ strongly increase, limiting the homogeneity length to less than $0.2 \mu \mathrm{m}$ (see Fig. 3.5B). Surprisingly, pumping at $\lambda=1.06 \mu \mathrm{m}$ in GaAs, as was proposed by Ref. [54] to optimize homogeneity, yields a short homogeneity length $\ell_{\text {hom }}=0.41$ $\mu \mathrm{m}$. This immediately shows, that GaAs at this pump frequency cannot be used 


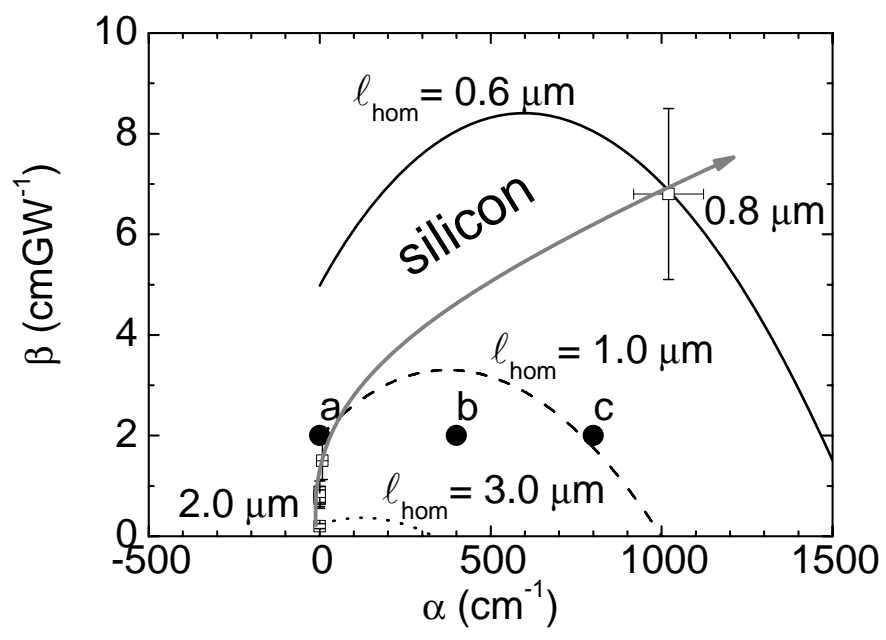

Figure 3.4: Homogeneity plot for Si. Contours of constant $\ell_{\text {hom }}$ are plotted in the $(\alpha, \beta)$ plane, defining regions in which homogeneous switching can be achieved. The generated carrier density is kept constant at $N_{e h}(0)=0.9 \times 10^{20} \mathrm{~cm}^{-3}$, enough for a $5 \%$ change in $n^{\prime}$ in Si. The solid curve corresponds to $\ell_{\text {hom }}=3.0 \mu \mathrm{m}$, the dashed curve to $\ell_{\text {hom }}=1.0 \mu \mathrm{m}$ and the dotted curve to $\ell_{\text {hom }}=0.63 \mu \mathrm{m}$ for bulk silicon. The open squares connected by the gray arrow are linear and two-photon coefficients for Si obtained from Fig. 3.2. The open circles $a, b$ and c correspond to the depth profiles $N_{\text {eh }}(z)$ plotted in Fig. 3.3. Calculation parameters: $\tau_{\text {pump }}=140 \mathrm{fs}$ and $\omega_{\text {pump }}=5000 \mathrm{~cm}^{-1}$.

in homogeneous switching experiments. From this analysis we learn that in GaAs the pumping frequencies must be reduced to half the band gap energy, where the two-photon absorption coefficient attains the lowest value.

We now want to derive the maximum homogeneous change in refractive index that can be obtained in Si. We therefore plot several equi-carrier density curves (described by Eq. 3.10) that correspond to a particular fixed homogeneity length. We choose a homogeneity length of $1.5 \mu \mathrm{m}$, since inside a typical photonic crystal with $25 \%$ filling fraction, the homogeneity length will be four times larger $(6 \mu \mathrm{m})$, thus fulfilling our homogeneity requirement. Fig. 3.6 displays contours for two different carrier densities $N_{0}=1 \times 10^{20} \mathrm{~cm}^{-3}$ and $2.4 \times 10^{20} \mathrm{~cm}^{-3}$.

In addition to the homogeneity contours, the two lowest curves in Fig. 3.6 indicate the minimum absorption coefficients for which a sufficiently large carrier density can be generated given the maximum available irradiance $I_{\max }=1 \mathrm{TWcm}^{-2}$ (see Chapter 4 ). These curves are described by: 


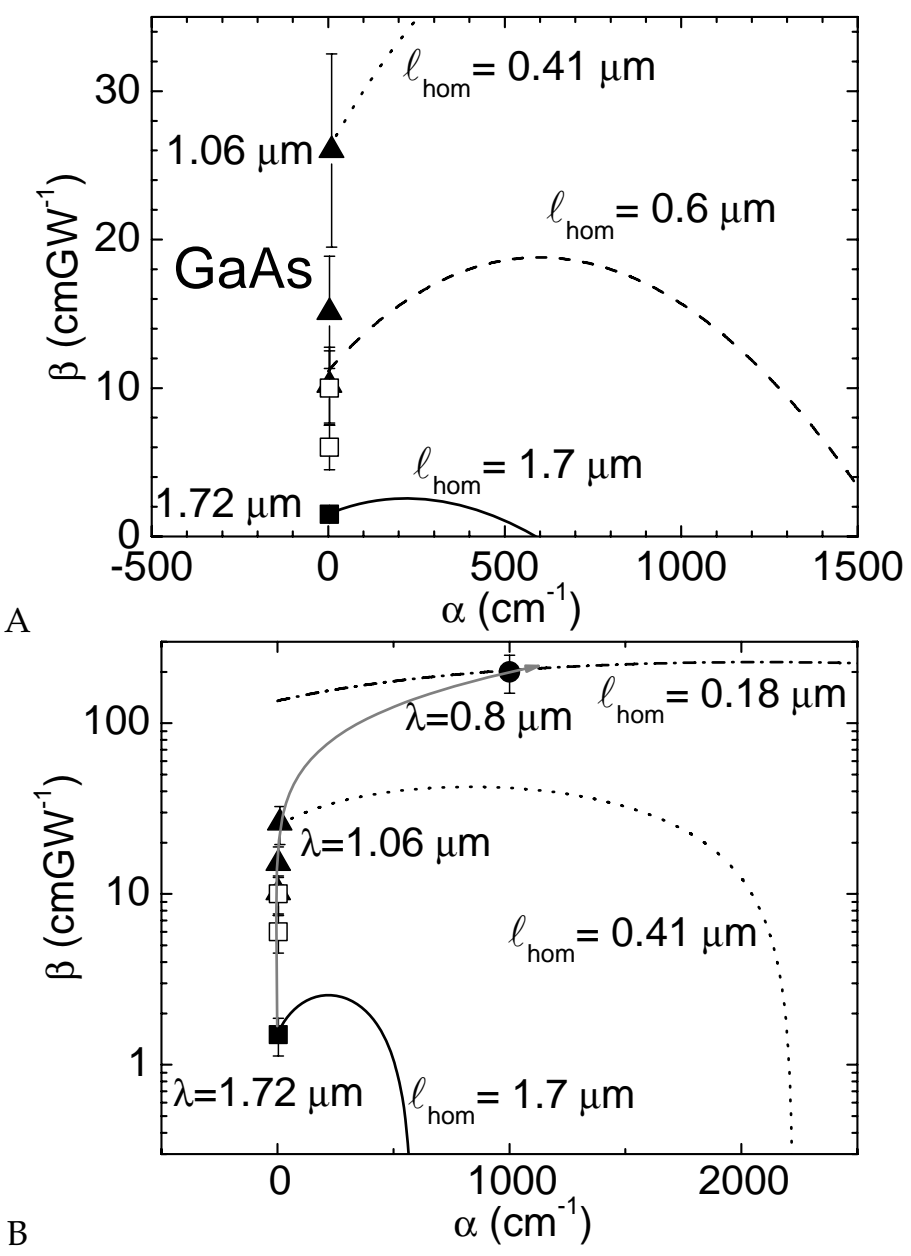

Figure 3.5: Homogeneity plot for $\mathrm{GaAs}$ in the two-photon absorption regime (A). A logarithmic ordinate was used in (B) to visualize the homogeneity at pump frequencies in both the linear as well as the two-photon absorption regime of $\mathrm{GaAs}$ in a single plot. Contours of constant $\ell_{\text {hom }}$ are plotted in the $(\alpha, \beta)$ plane, defining regions in which homogeneous switching can be achieved. The generated carrier density is kept constant at $N_{e h}(0)=0.35 \times 10^{20} \mathrm{~cm}^{-3}$, enough for a $5 \%$ change in $n^{\prime}$ in GaAs. The solid curve correspond to $\ell_{\text {hom }}=1.7 \mu \mathrm{m}$, the dashed curve to $\ell_{\text {hom }}=0.61 \mu \mathrm{m}$, the dotted curves to $\ell_{\text {hom }}=0.41 \mu \mathrm{m}$, and the dash-dotted curve to $\ell_{\text {hom }}=0.18 \mu \mathrm{m}$ for bulk $\mathrm{GaAs}$. Calculation parameters: $\tau_{\text {pump }}=140 \mathrm{fs}$ and $\omega_{\text {pump }}=5814 \mathrm{~cm}^{-1}$. The symbols are linear and two-photon coefficients for $\mathrm{GaAs}$ for pump wavelengths $\lambda=0.8 \mu \mathrm{m}$ (closed circle), $\lambda=1.06 \mu \mathrm{m}, \lambda=1.27 \mu \mathrm{m}$, and $\lambda=1.54 \mu \mathrm{m}$ (filled triangles) obtained from Refs. [97, 102, 104]. The three lowest data points were obtained by us through z-scan experiments at $\lambda=1.63 \mu \mathrm{m}$ (open squares) and $\lambda=1.72 \mu \mathrm{m}$ (filled square). 


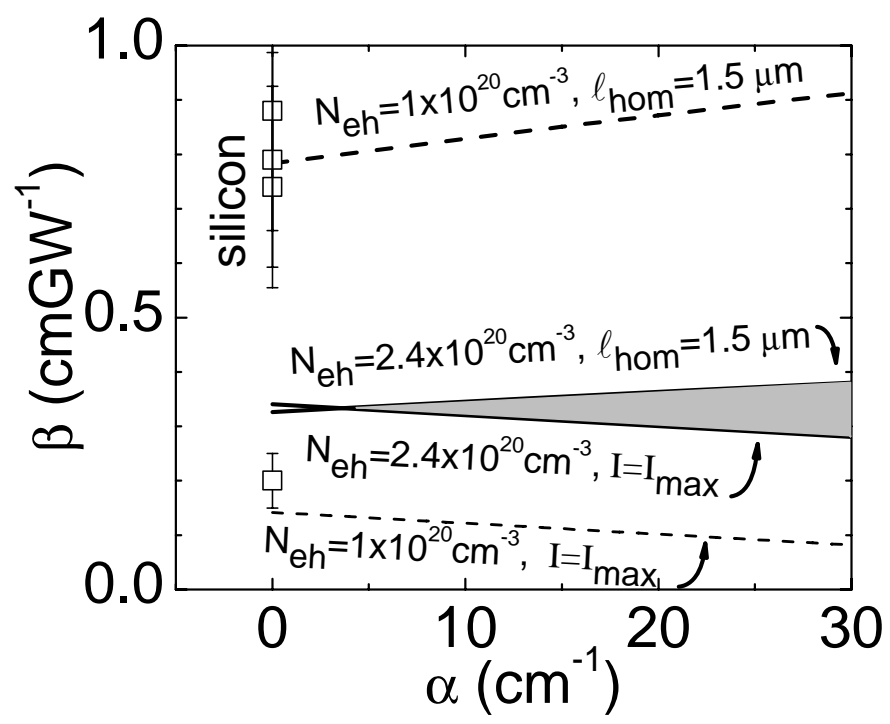

Figure 3.6: Carrier density contours for a particular bulk homogeneity chosen as $\ell_{\text {hom }}=1.5 \mu \mathrm{m}$. The contours correspond to two different values for $N_{0}$ : the dashed lines to $N_{0}=1.0 \times 10^{20} \mathrm{~cm}^{-3}$, and the solid lines to $N_{0}=2.4 \times 10^{20} \mathrm{~cm}^{-3}$. The upper one of each pair of curves corresponds to $\ell_{\text {hom }}=1.5 \mu \mathrm{m}$. The lower curve of each pair indicates the minimum absorption coefficients for which the given $N_{\text {eh }}(0)$ can be obtained without exceeding the maximum irradiance $I_{\max }=1 \mathrm{TWcm}^{-2}$. The closed squares connected by the dotted curve are the $\alpha$ and $\beta$ coefficients for Si obtained from Fig. 3.2. Pumping parameters: $\tau_{\text {pump }}=140 \mathrm{fs}, r_{\text {pump }}=75 \mu \mathrm{m}$ and $\omega_{\text {pump }}=5000 \mathrm{~cm}^{-1}$.

$$
\beta=\frac{2\left(\hbar \omega_{\text {pump }} N_{0}-\tau_{\text {pump }} I_{\text {max }} \alpha\right)}{\tau_{\text {pump }} I_{\text {max }}^{2}},
$$

which was obtained by solving Eq. 3.5 for a fixed carrier density $N_{0}$. For feasible switching experiments, the absorption coefficients must be in the shaded area to the right of the intersection of the two curves. As the carrier density and thus the refractive index change increases, the constant $\ell_{\text {hom }}$ line moves towards lower absorption coefficients, while the $I_{\text {max }}$ line moves towards higher values. As an example: for a carrier density of $2.4 \times 10^{20} \mathrm{~cm}^{-3}$, homogeneous switching can only be achieved for absorption coefficients within the shaded area in Fig. 3.6. It is seen that this area does not overlap with the trajectory of silicon parameters. Therefore, this carrier density is not achievable in Si for $\ell_{\text {hom }}=1.5 \mu \mathrm{m}$. With decreasing carrier density, the range between the curves will overlap the silicon parameter trajectory at some point. Such an intersection determines the upper limit to the carrier density 
(given $\ell_{\text {hom }}=1.5 \mu \mathrm{m}$ ) as well as the pump frequency that pertains to the relevant $(\alpha, \beta)$-point. For bulk silicon, this intersection is calculated to occur at $\mathrm{N}_{e h}=2.3 \times 10^{20}$ $\mathrm{cm}^{-3}$. The corresponding maximum homogeneous change in refractive index at $\omega_{\text {tele }}$ is as large as $13 \%$. We note that while such large refractive index changes can be achieved in bulk Si [73], the poly-crystalline samples discussed in Chapter 6 and refinvopals, the maximum achievable carrier density, is limited by the damage threshold to $\approx 5 \times 10^{19} \mathrm{~cm}^{-3}$.

The condition of a maximum irradiance $I_{\max }$ can be relaxed by choosing a smaller pump spot radius $\mathrm{r}_{\text {pump }}$. The assumed pump radius of $75 \mu \mathrm{m}$ provides a lateral homogeneity which is large compared to the homogeneity requirement (see Section 4.3), therefore we can choose a smaller $r_{\text {pump }}$ while maintaining sufficient lateral homogeneity. A higher pump irradiance would allow homogeneous switching experiments at even lower absorption coefficients, allowing larger changes of the refractive index. However, we must keep in mind that the probe absorption length $\ell_{\text {probe }}$ is inversely proportional to the induced carrier density, see Eq. A.9. For a refractive index change of $13 \%$, we predict the probe absorption length inside a photonic crystal to drop to $\ell_{\text {probe }}=30 \mu \mathrm{m}$, which may be sufficient to meet our third requirement for switching experiments in photonic crystals. At higher carrier densities however, the carrier absorption length will become too small to meet this requirement.

\subsection{Switching homogeneity in real photonic crystals}

The analysis in the previous section was done for bulk semiconductor materials. To obtain the homogeneity length in photonic crystals, the bulk absorption length is divided by the semiconductor filling fraction $\Phi$. In case of a $5 \%$ switch of the refractive index in a silicon photonic crystal with $\Phi=25 \%$ at $\omega_{\text {pump }}=5000 \mathrm{~cm}^{-1}$, the homogeneity length thus increases from $3.0 \mu \mathrm{m}$ to $12 \mu \mathrm{m}$, which is twice the homogeneity requirement of $6 \mu \mathrm{m}$. For a higher pump frequency of $12500 \mathrm{~cm}^{-1}$, we find a homogeneity length of $2.3 \mu \mathrm{m}$, which is too low. This illustrates that the homogeneity required for switching of the density of states in silicon photonic crystals can only be achieved with nonlinear absorption processes.

In the analysis so far, the extinction of pump light due to random scattering inside the photonic crystals was neglected. We now discuss how to incorporate inevitable scattering in photonic samples. Scattering is quantified by the mean free path $\ell_{m f p}$ : the characteristic length over which a coherent beam becomes diffuse. The homogeneity length of light inside a photonic crystal is related to $\ell_{m f p}$ and $\ell_{a b s}$ 
as

$$
\ell_{\text {hom }}=0.1 \times\left[\frac{1}{\ell_{a b s}}+\frac{1}{\ell_{m f p}}\right]^{-1} .
$$

In the limit of weak scattering, where $\ell_{m f p} \gg \ell_{a b s}$, discussed before in Eq. 3.12 reduces to $\ell_{\text {hom }}=0.1 \times \ell_{\text {abs }}$ (see Eq. 3.9). In the limit of strong scattering, where $\ell_{m f p} \ll \ell_{a b s}$, the homogeneity length becomes $\ell_{\text {hom }}=0.1 \times \ell_{m f p}$. As opposed to the adverse effect of scattering on the homogeneity in the z-direction, scattering will generally be favorable for lateral homogeneity, as pump light which is removed from the coherent pump beam is be scattered laterally.

Recently, our group has developed a quantitative model of scattering inside photonic crystals [17]. One of the main consequences of the model to switching is that the mean free path for pump light decreases with pump frequency squared $\omega_{\text {pump }}^{-2}$. The absolute value of the mean free path depends not only on frequency, but also on many properties of the crystal such as the unit cell size, the amount of disorder and the refractive index contrast. As an example, we apply the model to a silicon inverse opal photonic crystal with a lattice parameter of $a=1240 \mathrm{~nm}$, corresponding to a band gap frequency near $\omega_{\text {tele }}$. We assume combined size poly dispersity and lattice displacements of $2 \%$ (which is beyond the current state of the art). From the model of Ref. [17], we obtain a mean free path of 5.3 unit cells for pump light at $\omega_{\text {pump }}=12500 \mathrm{~cm}^{-1}$. The corresponding homogeneity length, dominated by $\ell_{m f p}$, would thus be 0.5 unit cells, well below our homogeneity requirement of 5 unit cells. For a 2.5 times lower pump frequency of $5000 \mathrm{~cm}^{-1}$, the model predicts a $(2.5)^{2}$ times larger mean free path of $\ell_{m f p}=33.3$ unit cells inside the crystal. In Eq. 3.12, we combine $\ell_{m f p}$ with the earlier obtained absorption length $\ell_{a b s}=116 \mu \mathrm{m}$ (= 93 unit cells), and we obtain a homogeneity length of $0.1 \times[1 / 93+$ $1 / 33]^{-1}=2.4$ unit cells. This result points out that even at pump frequencies near the two-photon absorption edge of silicon, the homogeneity requirement of 5 unit cells can not be met in silicon inverse opals with a lattice parameter of $a=1240 \mathrm{~nm}$.

The scattering model predicts that decreasing the unit cell size will result in higher homogeneity, as this will reduce the relative pump frequency $a \omega_{\text {pump. }}$. Therefore, we consider silicon inverse opals with a reduced lattice parameter of $a=900$ $\mathrm{nm}$, corresponding to a band gap near $8900 \mathrm{~cm}^{-1}$, just below the absorption edge of silicon. The scattering model predicts a larger mean free path for pump light with a frequency of $5000 \mathrm{~cm}^{-1}$ of $\ell_{m f p}=63$ unit cells. Using the absorption length of 116 $\mu \mathrm{m}\left(=129\right.$ unit cells), we obtain homogeneity length of: $\ell_{\text {hom }}=0.1 \times[1 / 129+1 / 63]^{-1}=$ 4.2 unit cells, close to our homogeneity requirement of 5 unit cells. For smaller refractive index changes of $\approx 1-2 \%$, relevant to our experiments in the thin film opals 
in Chapter 7, we estimate that our sample only marginally fulfills our homogeneity criterium. Since the homogeneity length is larger than the sample thickness, however $\left(\ell_{\text {hom }}>L\right)$, we still expect homogeneous changes.

Major improvements in switching homogeneity can be made by studying diamond-like structures [25, 51, 105], as the lattice parameter can be as low as $600 \mathrm{~nm}$ for a band gap near $\omega_{\text {tele }}$ in such crystals. In such structures, the scattering mean free path remains unchanged, apart from a constant prefactor of order unity that depends on the shape of the unit cell. We predict that reduced lattice parameters will decrease the mean free path sufficiently to allow homogeneous photonic density of states switching experiments. Homogeneous switching experiments on woodpile photonic band gap crystals are presented in Chapter 6.

\subsection{Conclusions}

We have discussed a nonlinear absorption model to describe the spatial homogeneity of optically generated electron-hole plasmas in semiconductors. We have introduced a general homogeneity diagram, which directly relates linear and twophoton absorption coefficients to the maximum homogeneity that can be achieved for any semiconductor. From such a diagram, we conclude that for density of states switching in silicon photonic crystals, the optimum carrier density is about $10^{20} \mathrm{~cm}^{-3}$. To obtain the required homogeneity, the absorption coefficients must be minimized, by a judicious choice of pump frequency. Due to peak irradiance limitations the lowest pump frequency that can be chosen for $\mathrm{Si}$ is around 5000 $\mathrm{cm}^{-1}$. For GaAs, the pump frequency must be chosen around $5814 \mathrm{~cm}^{-1}$.

We have discussed the effect of scattering in photonic crystals on the pumping homogeneity. The homogeneity of switched photonic crystals turns out to be limited by scattering. We conclude that the homogeneity condition can barely be met in silicon inverse opals. We predict that in diamond structures the relative pump frequency will be sufficiently small to allow homogeneous switching, even of the density of states. 



\section{Chapter}

\section{Instrumentation}

We describe our time resolved spectroscopy setup which can be tuned over broad frequency ranges. We demonstrate a new data acquisition method that allows for sensitive pump probe measurements, even while the laser output fluctuates. We show switched reflectivity data of a bulk silicon test sample.

\subsection{Pump probe setup}

The time-resolved optical measurements on photonic crystals were done with a pump-probe method [106] using a dedicated two-color pump-probe setup. Our laser system provides high power pulses at two independently tunable frequencies, allowing us to adjust the pump frequency to optimize the spatial homogeneity and the probe frequency to scan across broad photonic gaps. The setup is based on a regeneratively amplified Titanium Sapphire laser that emits short $120 \mathrm{fs}$ pulses at $\lambda=800 \mathrm{~nm}$ with a pulse energy of $1 \mathrm{~mJ}$ at a repetition rate of $1 \mathrm{kHz}$ (Spectra Physics Hurricane). This laser drives two optical parametric amplifiers (OPA, Topas 800fs) shown schematically in Fig. 4.1, that serve as pump and probe. The output frequencies of the OPAs are computer controlled, and can be continuously tuned between 3850 and $21050 \mathrm{~cm}^{-1}$. The delay between pump- and probe pulse was set by a $40 \mathrm{~cm}$ long optical delay line with a time resolution of $\Delta t=10 \mathrm{fs}$. Since the delay time is also computer controlled, we can scan the reflectivity spectrum as a function of frequency at a chosen time delay after the pump pulse.

The excitation of carriers at pump frequencies near the two-photon absorption edge of semiconductors requires a high pump irradiance in the range of 10-300 


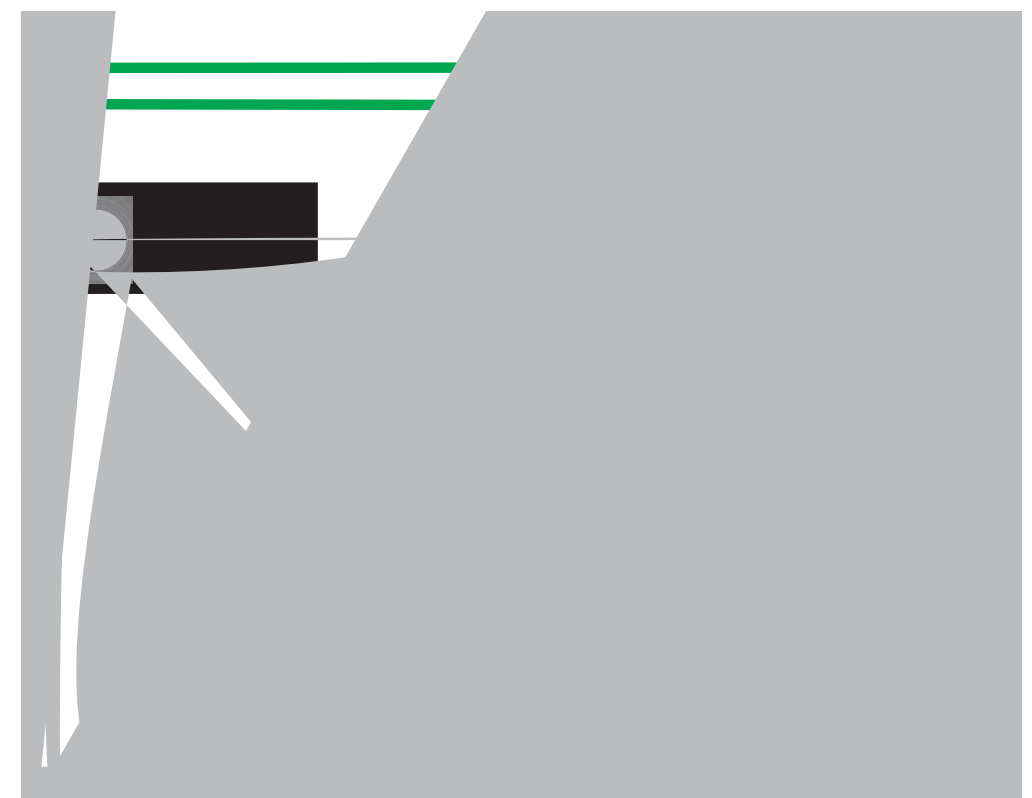

Figure 4.1: Schematic drawing of the setup. Pulses ( $\tau=120 \mathrm{fs}, \omega=12500 \mathrm{~cm}^{-1}, E=1 \mathrm{~mJ}$ ) from a regeneratively amplified Ti:Saph amplifier (not shown) drive two OPAs. The output frequency of both OPAs is computer controlled, and tunable from 3850 to $21050 \mathrm{~cm}^{-1}$. The pump pulse passes through an optical delay line with minimum time step of $10 f_{s}$. Both pump and probe beam pass through a chopper wheel that is synchronized to the laser output (see Fig. 4.3). Both pump and probe beam are focussed on the same spot on the sample. Two InGaAs photodiodes are used to monitor the output variation of the OPAs as well as the reflected signal. The reflectivity from the sample is measured by a third InGaAs photodiode. Three boxcar averagers are used to hold the short output pulses of each detector for $1 \mathrm{~ms}$, allowing simultaneous acquisition of separate pulse events of all three detector channels.

$\mathrm{GWcm}^{-2}$, depending on the material and the pump frequency chosen. Since both OPAs have a conversion efficiency that exceeds $30 \%$, a pulse energy $E_{\text {pulse }}$ of at least $20 \mu \mathrm{J}$ is available over the whole frequency range. The output of our OPAs consists of $\tau_{p}=140 \pm 10 \mathrm{fs}$ (measured at $\omega=7690 \mathrm{~cm}^{-1}$ ) pulses with a spectral shape of the output spectrum that was measured to be Gaussian with a frequency independent linewidth $\Delta v / v=1.44 \pm 0.05 \%$. We deduce the time-bandwidth product to be $\tau_{p} \Delta v=$ $0.47 \pm 0.05$, in good agreement with the Fourier limit for Gaussian pulses $\left(\tau_{p} \Delta v=\right.$ 0.44 [107]). We take a Gaussian intensity envelope of the pulse: 


$$
P(t)=P_{\max } e^{-2\left(\frac{t}{\tau_{p}}\right)^{2}},
$$

where $P_{\max }=\left(E_{\text {pulse }} / \tau_{p}\right)(\sqrt{2 / \pi})$ is the peak power. Experimentally, we obtain the $P_{\max }$ inside the sample by subtracting the pump reflectivity at the sample interface:

$$
P_{\text {max }}=P_{\text {ext }}(1-R),
$$

where $P_{\text {ext }}$ it the external pump power, and $\mathrm{R}$ is the measured reflectivity of the pump beam at the sample interface at $\omega_{\text {pump }}$. Both pump and probe beams were focused onto the sample at a small numerical aperture $N A=0.02$. The pump beam profile was characterized by a knife edge scan shown in Fig 4.2. The resulting curve corresponds well to the analytical result for a Gaussian beam profile with a width $w_{\text {pump }}=113 \pm 5 \mu \mathrm{m}$. We therefore describe the spatial irradiance distribution in the focus with a Gaussian:

$$
I(x, y)=I_{0} e^{-2 \frac{x^{2}+y^{2}}{w_{p}^{p} \text { pump }}},
$$

where $I_{0}=\left(P_{\max } / w_{\text {pump }}^{2}\right)(2 / \pi)$ is the peak irradiance in the center of the focus. Even with a large pump focus of $w_{\text {pump }}=113 \pm 5 \mu \mathrm{m}$, the maximum peak irradiance $I_{\max }$ that can be obtained in our setup still exceeds exceeds $1 \mathrm{TWcm}^{-2}$. This large excess irradiance indicates that it is feasible to switch an even larger part of a sample, or to use less powerful lasers, which is important to allow for possible applications.

The probe beam was focussed to a Gaussian spot of typically $w_{\text {probe }}=20 \pm 5 \mu \mathrm{m}$. Since the probe focus is much smaller than the pump focus we provide a good lateral homogeneity. In all experiments, we explicitly ensured that only the central flat part of the pump focus is probed by testing with a Si wafer.

\subsection{Data acquisition method}

There are several important issues that limit the speed and accuracy of our pump probe setup. First of all, since our experiments intrinsically depend in the magnitude of the irradiance, we are sensitive to pulse-to-pulse variations of our laser. Our OPAs show typical variations of $7 \%$ in the worst case near the degeneracy point near $\lambda=1600 \mathrm{~nm}$, and are stable to $2 \%$ near $\lambda=1300 \mathrm{~nm}$. To improve the signal-to-noise to better than the laser stability, it is thus important to probe individual pulse events so that pulse selection can be performed. Secondly, since our laser runs at a low repetition rate of $1 \mathrm{kHz}$, long integration times are required to sufficiently reduce the error in probe reflectivity signals. Thirdly, scattered light 


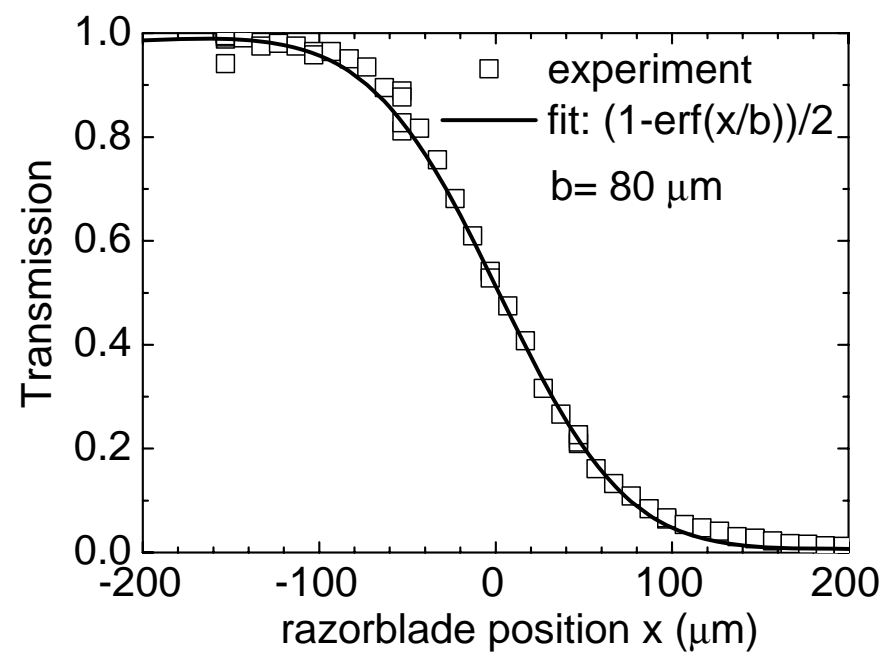

Figure 4.2: Spatial characterization of the pump beam at $\omega_{\text {pump }}=6450 \mathrm{~cm}^{-1}$ by a knife-edge measurement at the focus of a $f=150 \mathrm{~mm}$ achromatic lens. A razor blade was scanned through the focus. The corresponding transmission was measured with a powermeter, and normalized to the maximum transmission (symbols). The data is in good agreement with the analytical result $T(x)=(1$-er $f(x / b)) / 2$ for a Gaussian beam profile, where erf represents the error function. The width parameter $b=80 \mu \mathrm{m}$ yields a Gaussian width $w_{\text {pump }}=\sqrt{2} b=113 \mu \mathrm{m}$.

from the intense pump pulses contributes to the background signal of our probe detector. Often, the background level was found to be larger than the reflectivity changes of the samples under study. To resolve these issues, we have developed a versatile measurement scheme to subtract the pump background from the probe reflectance signal, and to compensate for pulse-to-pulse variations in the output of our laser.

The irradiance of each pump and probe pulse is monitored by two InGaAs photodiodes and the reflectivity signal is measured by a third InGaAs photodiode, shown as black squares in Fig. 4.1. Three boxcar averagers are used to hold the short output pulses of each detector for $1 \mathrm{~ms}$, allowing simultaneous acquisition of separate pulse events of all three detector channels by a data acquisition card. Both pump and probe beam pass through a chopper whose frequency is synchronized to the repetition rate $f=1 \mathrm{kHz}$ of the laser. The alignment of the two beams on the chopper blade is such that each millisecond, pump and probe beam are blocked or unblocked in a different permutation, see Fig. 4.3. In one sequence of four consecutive laser pulses, both pumped reflectivity (a), linear reflectivity (d), 

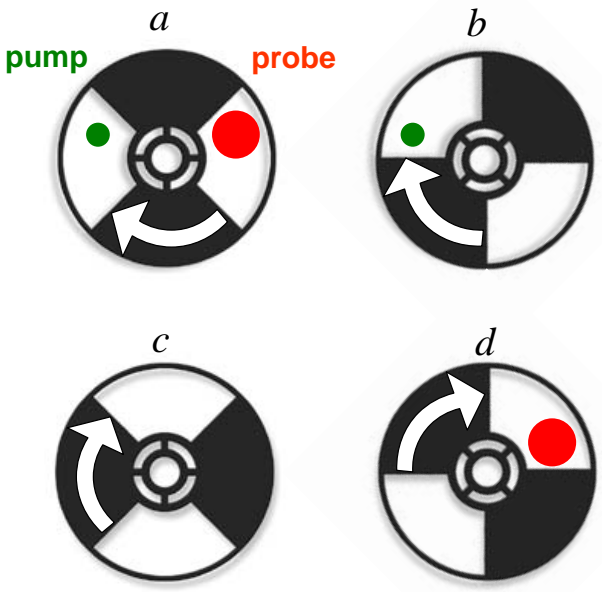

Figure 4.3: Schematic drawing illustrating the alignment of the pump-en probe beams onto the chopper blade. The rotation of the chopper wheel is synchronized to the laser output. One full revolution of the chopper blade takes $8 \mathrm{~ms}$, such that that for each pulse event, pump and probe beam are blocked or unblocked in a different permutation. In one sequence of four consecutive laser pulses, both excited reflectivity (a), linear reflectivity (d), and two background measurements $(b, c)$ are collected. In this flexible measurement scheme, detector signals for each pulse event are collected, allowing various data processing routines such as automatic background substraction and the selection of pulses within a certain pump energy range after the experiment.

and two background measurements $(b, c)$ are collected. The detector signals for 


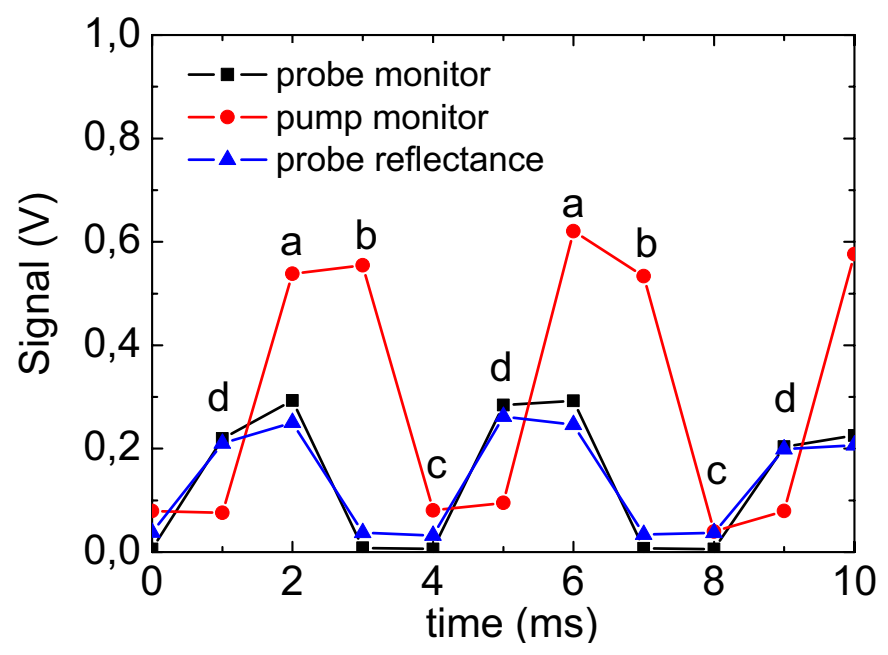

Figure 4.4: Time traces of the boxcar output signals for probe monitor, pump monitor, and probe reflectance. The sample was a GaAs/AlAs distributed Bragg reflector, the experimental conditions were the same as in the experiment in Fig. 5.3A. The pump irradiance was $\approx 100 \mathrm{GWcm}^{-2}$ the probe frequency was $\omega=6700 \mathrm{~cm}^{-1}$. The switched reflectivity is roughly $10 \%$ lower than the unswitched reflectivity. Each datapoint in the plot corresponds to a single pulse event. The letters $a, b, c$, and $d$ correspond to the chopper position during each event (see Fig. 4.3).

At each setting of the delay stage and both OPAs, all detector signals from $4 \times 250$ pulse events were collected and stored. The probe reflectance signal for $4 \times 250$ pulse events of a typical experiment is plotted versus probe monitor signal in Figure 4.5. Both signals show a variation as a result of the pulse-to-pulse variations of the laser. The datapoints constitute two separate lines whose slopes correspond to the unpumped and pumped reflectance of the sample. To exemplify the noise reduction of our method, we have chosen a data set during which the alignment of the pump laser was not optimized and pulse-to-pulse variations of the probe signal were larger than normal, amounting to a relative standard deviation $\sigma_{S D \text {, probe }}=13 \%$. The corresponding standard error in the mean detector signal is $\delta \mathrm{R} / \mathrm{R}=\sigma_{S D \text {, probe }} / \sqrt{N}=$ $13 \% / \sqrt{250}=0.8 \%$, which is relatively large compared to the effects that we wish to study. We therefore use an automated data processing routine to process the probe reflectance and probe monitor data to increase the signal-to-noise ratio. From the entire data set, the averages of the background levels (b) and (c) were determined, and subtracted from the pumped (a) and unpumped (d) reflectance data respectively. The resulting background-subtracted reflectance signal was divided by the corresponding monitor signal to compensate for intensity variations in the output 
of the laser. Through this procedure, the RMS variation in the unpumped reflectivity that was found from the data in Fig. 4.5 was strongly reduced to $\sigma_{S D \text {,probe }}=$ $1.1 \%$. The resulting standard error in the probe reflectivity was improved tenfold to $\delta \mathrm{R} / \mathrm{R}=0.07 \%$, even if the laser is not running optimally. Our scheme allows a sensitive measurement of the reflectivity and of small reflectivity changes, while maintaining an acceptable measurement time of about 1 second per frequencydelay setting. We attribute the remaining noise to uncorrelated electronic noise in the detection system.

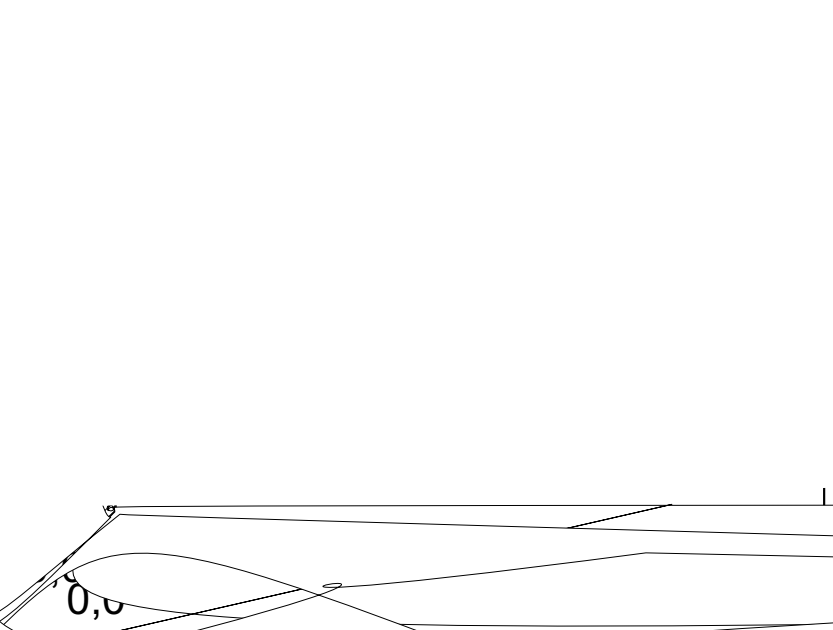

Figure 4.5: Reflectance signal versus monitor date for 1000 pulse events of the data set shown in Fig. 4.4 displayed as a scatter plot. The 250 unpumped reflectivity datapoints (d) constitute a line, indicating that that variations in monitor and reflectance signal are strongly correlated. The slope of the line is proportional to the reflectivity of the sample. The pumped datapoints (a) form a line with a reduced slope, due to the reflectivity decrease of about $\delta R / R=10 \%$ in the switched sample. Both background data sets $(b)$ and $(c)$ tend to the origin of the plot as it should in absence of offsets. Note that the small offset in the signals is automatically removed in the data processing routine.

Pulse to pulse variations in the pump energy are a more subtle issue, since such fluctuations will often propagate in a nonlinear, and sometimes unpredictable way in the reflectivity change $\Delta R / R$ of the sample. The open circles in Fig. 4.5 correspond to the switched reflectance data. The slope of the line that is formed by these data points is reduced by about $10 \%$ compared to the unswitched data (closed squares). The corresponding to a reflectivity decrease is equal to $\Delta R / R=$ $10 \%$. We also observe that the line is about twice as broad as the line corresponding 
to the unpumped data. We attribute the broadening to pulse to pulse variations of the pump.

In the example in Fig. 4.5 the standard deviation of the pump pulse energy $\sigma_{S D, p u m p}=12 \%$, from which we deduce a standard error in the pump irradiance $\delta I_{0} / I_{0}=\frac{\sigma_{S D, \text { pump }}}{\sqrt{N}}=0.8 \%$. The error in the reflectivity change $\Delta \mathrm{R} / \mathrm{R}$ due to the pulse-topulse irradiance fluctuations is equal to $\left.\delta(\Delta R / R)=2 \delta I_{0} / I_{0}\right)(\Delta R / R)$, where the factor 2 is due to the quadratic dependence of $\Delta \mathrm{R} / \mathrm{R}$ on $I_{0}$ for a two-photon process. Using the reflectivity change in the data in Fig. $4.5(\Delta R / R=10 \%)$ we obtain the pump contribution to the standard error in $\Delta R / R$ to be $\delta(\Delta R / R)=0.16 \%$. The error in reflectivity changes $\Delta R / R$ also contains a contribution of the fluctuations in the probe pulse that were discussed before. The error due to the probe variation is equal to $\sqrt{2}(\delta \mathrm{R} / \mathrm{R})=0.1 \%$, since the two independent error in the pumped-and unpumped datasets are added. We calculate the total error by adding the contributions of both probe and pump variations. We obtain a standard error:

$$
\delta\left(\frac{\Delta R}{R}\right)=\sqrt{2} \frac{\sigma_{S D, \text { probe }}}{\sqrt{N}}+\frac{\Delta R}{R} \frac{\sigma_{S D, p u m p}}{\sqrt{N}}=0.26 \%,
$$

which is sufficiently sensitive for our switching experiments. In summary, our data acquisition method allows a sensitive measurement of reflectivity changes, at short integration times of about $1 \mathrm{~s}$, even if the laser is not running optimally.

In some applications, an even higher sensitivity is required. Fortunately, pumpmonitor detector signals for each individual pulse event are stored. It is thus possible to reduce the pump term in Eq. 4.4 by selecting pump pulses within a certain narrow energy range after the experiment, at the price of longer integration times. This procedure was applied in z-scan measurements (see Appendix C), where pump stability is essential for the correct interpretation of the experimental data.

\subsection{Ultrafast switching of a bulk Si wafer}

\subsubsection{Temporal behavior}

An example of a carrier induced change of refractive index in bulk silicon is given in Fig. 4.6 (upper panel). In this experiment, a powerful ultrashort pump pulse was focussed to a spot with radius $\mathrm{w}_{\text {pump }}=70 \pm 10 \mu \mathrm{m}$, resulting in a peak irradiance at the sample interface of $\mathrm{I}_{0}=115 \pm 40 \mathrm{GWcm}^{-2}$. The reflectivity of a weaker probe pulse with a smaller spot radius of $\mathrm{w}_{\text {probe }}=20 \pm 5 \mu \mathrm{m}$ was measured in the center of the pumped spot at different time delays with respect to the pump pulse. The 
scan in Fig. 4.6 (upper panel) shows that the reflectivity of the sample changes from $32 \%$ to $28 \%$. The $10 \%-90 \%$ rise time is 230 fs, clearly an ultrafast change in $n^{\prime}$. From Fresnel's formula we find the refractive index change to be more than $10 \%$. Eq. A.7 was used to estimate the corresponding generated carrier density at the interface to be high: $N_{e h}=1.6 \times 10^{20} \mathrm{~cm}^{-3}$. The lower panel shows the intensity autocorrelation function (ACF) of the pump pulses. The full width half maximum (FWHM) of $200 \mathrm{fs}$, we therefore conclude that the free carriers have been generated almost instantaneously.

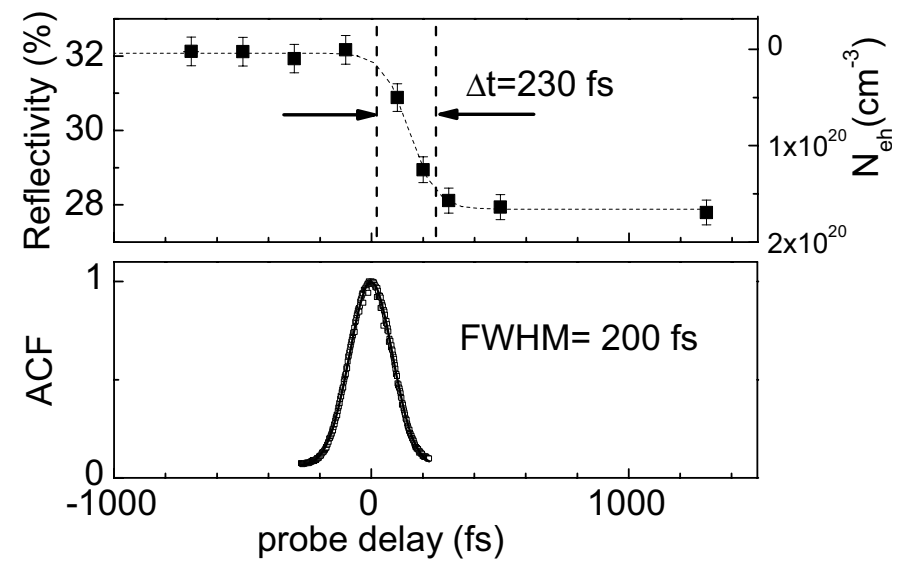

Figure 4.6: Time resolved reflectivity measurement on bulk Si, pumped at $\omega_{\text {pump }}=12500 \mathrm{~cm}^{-1}$, pulse energy $E_{\text {pump }}=2.0 \pm 0.1 \mu \mathrm{J}$, pulse duration $\tau_{\text {pump }}=120 \pm 10 \mathrm{fs}_{\mathrm{s}} w_{\text {pump }}=70 \pm 10 \mu \mathrm{m}$ and peak irradiance $115 \pm 40 \mathrm{GWcm}^{-2}$ (upper panel). The reflectivity of a probe with $\omega_{\text {probe }}=7692 \mathrm{~cm}^{-1}, w_{\text {probe }}=20 \pm 10$ $\mu \mathrm{m}$ and $\tau_{\text {probe }}=120 \pm 10 \mathrm{fs}$ decreases from $32 \%$ to $28 \%$, corresponding to a calculated carrier density $N_{e h}=1.6 \times 10^{20} \mathrm{~cm}^{-3}$ at the surface of the sample (see right-hand scale). The time difference between $10 \%$ and $90 \%$ of the total change is $230 \pm 40 \mathrm{fs}$. The lower panel shows the irradiance autocorrelation function (ACF) of the pump pulses. The full width half maximum (FWHM) of $200 \mathrm{fs}$ corresponds to a Gaussian pulse width of $\tau_{p}=120 \pm 10 \mathrm{fs}$.

Fig. 4.7 shows reflectivity from an extended probe delay range of -12 to +5 ps. Quite remarkably, at a negative probe delay of $8.6 \mathrm{ps}$, we observe an additional large step in the reflectivity from $38 \%$ to $32 \%$. We can identify three distinct probe delay regimes $\mathrm{A}, \mathrm{B}$, and $\mathrm{C}$, which are separated by two large steps in the reflectivity. The time difference between the first and second step is $8.6 \pm 0.5 \mathrm{ps}$, this value corresponds well to twice the optical thickness of the wafer $\left(2 L n_{s i} / c=8.3 \pm 0.1\right.$ ps) and is much longer than the pulse duration.

To visualize the observer unusual time dependence, we show in Fig. 4.8 snap- 


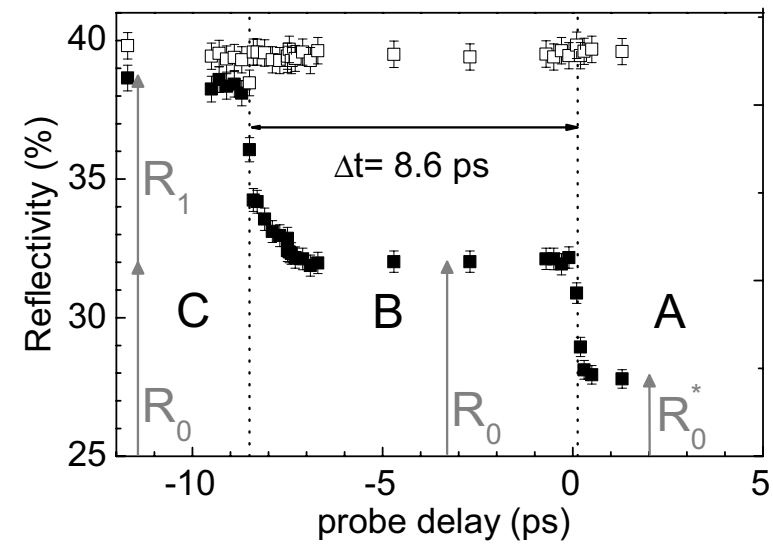

Figure 4.7: Time resolved reflectivity of a switched double side polished Si wafer. Unswitched reflectivity (open squares) and switched reflectivity (closed squares) are plotted over en extended range of probe delays compared to Fig. 4.6. Surprisingly, at a negative probe delay of $8.6 \mathrm{ps}$, a large step in the reflectivity from $38 \%$ to $32 \%$ appears. At zero probe delay the reflectivity decreases further from $32 \%$ to $28 \%$. The time difference between the first and second step in reflectivity (indicated by dotted lines) is $8.6 \pm 0.5 \mathrm{ps}$, which roughly corresponds to twice the optical thickness of the wafer $(8.3 \pm 0.1 \mathrm{ps})$. We identify three different probe delay regimes $A, B$, and $C$, that are explained in the schematic plot in Fig. 4.8.

shots of the reflected irradiance, taken at the moment that the pump pulse switched the front face of the wafer. The reflectivity of the wafer is built up from multiply reflected pulses from front and back surface of the wafer, which are indicated by $R_{0}, R_{1}$, and $R_{2}$. The magnitude of each successive reflection is given by $R_{m}=\left(1-R_{0}\right)^{2} R_{0}^{2 m-1}$. We neglect any further reflections in our analysis. It is important to note that each subsequent reflection $R_{m}$ is delayed with respect to $R_{0}$ by $\Delta \mathrm{t}_{m}=\mathrm{m} 2 \mathrm{~L} n_{S i} / c$, where $n_{S i}=3.5$ is the refractive index of $\mathrm{Si}$ at $\lambda=1300 \mathrm{~nm}$ [96], and $L=356 \pm 5 \mu \mathrm{m}$ is the thickness of the wafer.

The pump conditions in the experiment in Fig. 4.6, result in an inhomogeneous, dense carrier plasma near the front face of the wafer. From our homogeneity plot for silicon, see Fig. 3.4, we estimate a short homogeneity length of less than $\ell_{\text {hom }}<0.5$ $\mu \mathrm{m}$. Free-carrier absorption and diffraction from the dense plasma results in a strongly attenuated transmission. The plasma thus acts as an ultrafast shutter that blocks internally reflected pulses $R_{m}$ that arrive at the front face after the switching. 

sured the relative change in probe reflectivity from a bulk silicon sample pumped in the two-photon absorption regime at $\omega_{\text {pump }}=6250 \mathrm{~cm}^{-1}$. The probe frequency $\omega_{\text {probe }}$ is $7692 \mathrm{~cm}^{-1}$, with a focus size of $w_{\text {probe }}=25 \pm 5 \mu \mathrm{m}$. The probe delay is fixed at $10 \mathrm{ps}$ to avoid transient effects. The pump focus position is varied with respect to the probe focus by adjusting the pump mirror with a micrometer drive see Fig. 4.9.

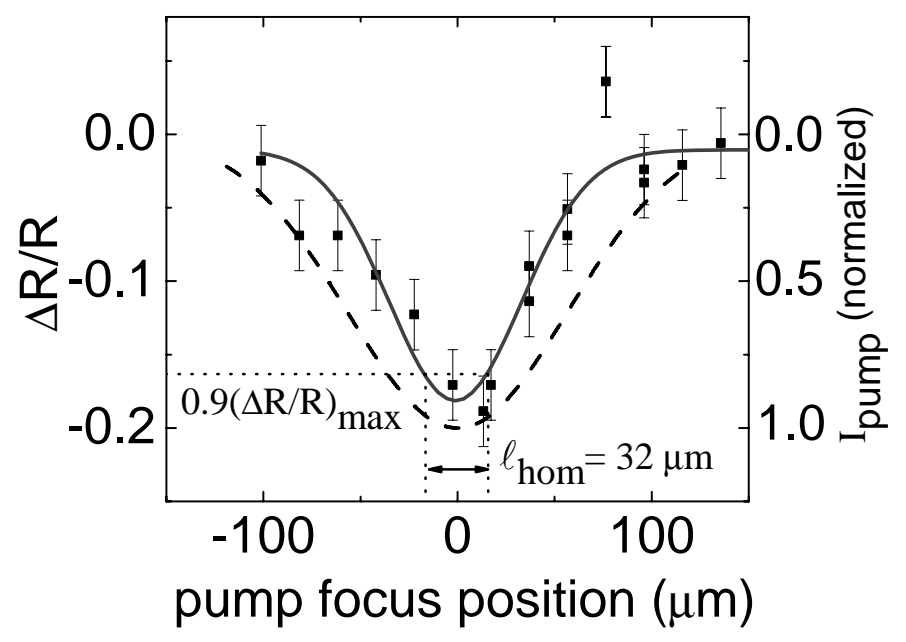

Figure 4.9: Relative probe reflectivity measurement on a bulk Si sample for different lateral positions of the probe focus with respect to the probe focus. The probe delay is fixed at $10 \mathrm{ps}$. Pumping parameters: $\tau_{\text {pump }}=120 \mathrm{fs}, \omega_{\text {pump }}=6250 \mathrm{~cm}^{-1}, E_{\text {pump }}=10.8 \mu \mathrm{J}, w_{\text {pump }}=68 \mu \mathrm{m}, \tau_{\text {pump }}=120 \mathrm{fs}_{\mathrm{s}}$, the pump irradiance is $I_{0}=485 \pm 150 \mathrm{GWcm}^{-2}$. At this frequency, $\beta=0.8 \pm 0.1 \mathrm{cmGW}^{-1}$ and $\alpha=0$ (see Appendix (). By combining Eq. 3.7 and Eq. 3.9, using $\alpha=0$, we obtain the corresponding homogeneity length $\left.\ell_{\text {hom }}=0.1 / \beta I_{0}=2.5 \pm_{-0.8}^{+2.0}\right) \mu \mathrm{m}$. Probe parameters: $\omega_{\text {probe }}=7692 \mathrm{~cm}^{-1}, w_{\text {probe }}=25 \pm 5 \mu \mathrm{m}$. The squares are the measured datapoints, fitted with a Gaussian curve width of $68 \pm 10 \mu \mathrm{m}$ (solid curve). The horizontal dotted line indicates the level where the change in reflectivity has decreased by $10 \%$. From the vertical dotted lines, which indicate the intersections of the $90 \%$ line with the Gaussian fit of the data, we obtain a lateral homogeneity length of $32 \mu \mathrm{m}$, illustrating the excellent lateral homogeneity. The dashed curve indicates the measured width of the pump focus.

The measured relative reflectivity shows a minimum at the center of the pump distribution, and decreases away from the center. The reflectivity data was fitted by a Gaussian curve (solid curve). For comparison, the dashed line in the graph is drawn to indicate the width of the pump irradiance distribution. The radius of this measured reflectivity minimum is $34 \pm 5 \mu \mathrm{m}$, which is considerably smaller than the $\mathrm{w}_{\text {pump }}$. To obtain the lateral homogeneity length, we have determined the maximum lateral distance from the pump focus center for which $\Delta R / R$ remains 
within $10 \%$ of its maximum value (dotted lines). This distance turns out to be $16 \pm 2 \mu \mathrm{m}$ in both directions, corresponding to a lateral homogeneity length of $32 \pm 4$ $\mu \mathrm{m}$, which is much greater than the $6 \mu \mathrm{m}$ which we required for switching of the density of states. Therefore we conclude that the high output power of our OPAs allows for the use of large pump foci, resulting in excellent lateral homogeneity.

\subsection{Conclusions}

We have built a two-color pump-probe setup that provides high energy, ultrashort laser pulses at optical frequencies in the range between 3850 and $21050 \mathrm{~cm}^{-1}$. A versatile measurement scheme was developed to automatically subtract the pump background from the probe signal, and to compensate for possible pulseto-pulse variations in the output of our laser. We deduce a tenfold improvement of the precision of the setup, allowing a measurement accuracy of $\Delta R=0.07 \%$ in $1 \mathrm{~s}$ measurement time. As an example, we have demonstrated a large and ultrafast change of the reflectivity of a bulk $\mathrm{Si}$ wafer, in which free carriers are homogeneously excited. 



\section{Chapter}

\section{Ultrafast switching of the photonic strength of III-V semiconductor Bragg reflectors}

We have used optical free-carrier generation by ultrashort laser pulses to switch the photonic strength of of 25-pair thick GaAs-AlAs photonic structures. We selectively and spatially homogeneously excite free carriers in the GaAs layers. We observe both a narrowing of the stop gap, which is caused by the reduced photonic strength of the structure, as well as a blue shift in the center position of the band, which is related to a reduced average refractive index. The resulting absolute reflectivity changes in the stop band were studied as a function of both probe frequency and delay time. We have observed large and ultrafast reflectivity decreases of up to $\Delta R>40 \%$ on the red edge of the stop band, as well as large increases of up to $\Delta R>8 \%$ on the blue edge of the stop band. The measured spectra compared well to transfer matrix theory that takes into account the nonlinear absorption of pump light, the bandwidth of our laser system. The refractive index of the excited GaAs layers is described by a Drude model.

\subsection{Introduction}

Switching experiments in photonic crystal are very exciting, but at the same time intrinsically difficult as they involve nonlinear optical measurements. Furthermore, the interpretation of experiments on complex nano-structures is more difficult 
than experiments on homogeneous media, since in practice, realized structures deviate from the ideal structure. Therefore, it is well worth to perform switching experiments on one-dimensional structures that are simple enough to allow an as exact as possible theoretical description, while still possessing characteristic photonic properties. Therefore we study in this Chapter switching experiments on semiconductor dielectric mirrors, also known as distributed Bragg reflectors.

There are three important reasons why Bragg reflectors are highly suitable study materials to investigate switching of photonic crystals.

Firstly, an important similarity to photonic crystals is that the interaction strength of Bragg reflectors with incoming light is also gauged by the photonic strength $S$ (see Section 1.1), which is defined as the ratio of the polarizability of each structural unit cell to the physical volume $[14,15]$. In any Bragg reflector, $S$ depends on the thickness of the high index $d_{h}$ and the low index $d_{\ell}$ layers, as well as on their refractive indices $n_{h}$ and $n_{\ell}$. We can rewrite Eq. 1.3 as:

$$
S=\left|n_{h}-n_{\ell}\right|\left(\frac{1}{n_{\ell}}+\frac{1}{n_{h}}\right) \frac{1}{\pi} \sin \left(\frac{\pi d_{\ell}}{d_{\ell}+d_{h}}\right) .
$$

In the special case of a distributed Bragg reflector (DBR), each layer has a constant optical thickness $d_{h, \ell}=\lambda_{c} / 4 n_{h, \ell}$ which is optimized for photonic strength. Consequently, Eq. 5.1 can be approximated by

$$
S \approx \frac{4}{\pi}\left|\frac{n_{G a A s}-n_{A l A s}}{n_{G a A s}+n_{A l A s}}\right| .
$$

Thus the photonic strength is proportional to the refractive index contrast between the layers [4] which we will switch on ultrafast time scales.

Secondly, the Bragg reflectors under study exhibit strong Bragg diffraction and stop bands, similar to 2D and 3D photonic crystals. An important difference, however, is that a stop band occurs for one propagation direction only, and therefore the structures do not possess a true gap in the LDOS. Nevertheless, even in onedimensional structures, sizable variations of the LDOS with position have been predicted for specific dipole orientations [108].

Thirdly, the layers in the structure are grown by molecular beam epitaxy (MBE), yielding well defined atomic lattices [109]. Consequently, the linear optical properties of the materials used in the Bragg reflectors are comparable to their well-known bulk values [96]. Furthermore, a simple Drude description can be used to model the nonlinear optical response of the materials, greatly facilitating the interpretation of our results. In contrast, the 3D photonic crystals discussed in Chapter 6 and 7 consist of poly-crystalline materials whose optical and electronic properties 
differ from bulk [ 


$$
\Delta \omega(\omega)=\frac{1}{2 N\left(d_{G a A s} n_{G a A s}^{\prime}(\omega)+d_{A l A s} n_{A l A s}^{\prime}(\omega)\right)},
$$

where $\Delta \omega(\omega)$ is the fringe spacing, and $N$ is the number of pairs of layers in the structure. An exact transfer matrix model that includes the dispersion of GaAs [96] is shown as a curve in Fig. 5.1. From our model we deduce a Fabry-Pérot spacing of $\Delta \omega=267 \pm 4 \mathrm{~cm}^{-1}$, in excellent agreement with our experimental data.

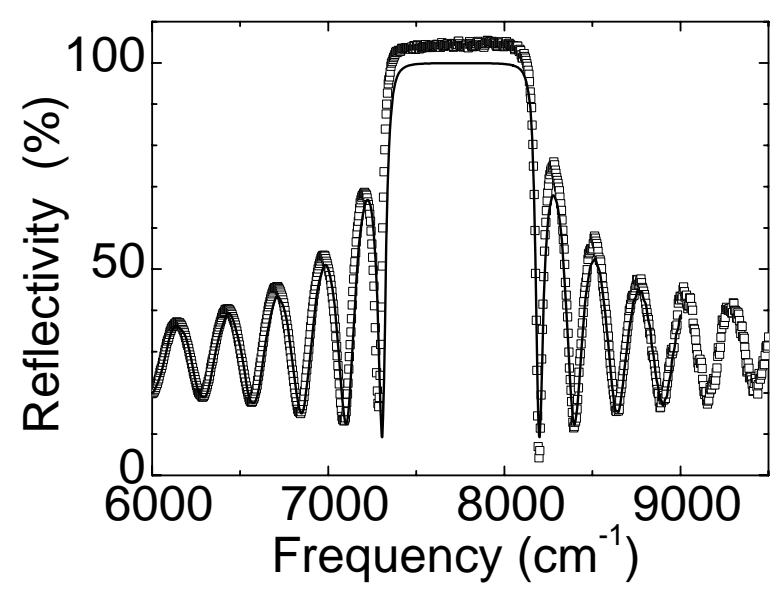

Figure 5.1: Linear FT-IR reflectivity measurements at perpendicular incidence of a 25 pair thick GaAs-AlAs DBR sample with center frequency $\omega_{c}=7737 \mathrm{~cm}^{-1}$ (symbols). The measured maximum reflectivity $R=104 \pm 5 \%$ slightly exceeds $100 \%$, probably due to a fixed scaling error in the gold reference. The width of the stop band is $\Delta \omega / \omega=11.3 \%$. The curve indicates an exact transfer matrix calculation that includes the dispersion of GaAs [96]

\subsubsection{Photonic strength}

The width of the stop band of the $\lambda_{c}=1300 \mathrm{~nm}$ sample in Fig. $5.1 \Delta \omega / \omega=11.2 \pm 0.2 \%$ FWHM is directly related to the photonic interaction strength $S$, see Section 1.1.3. For our DBR samples, consisting of GaAs and AlAs layers, Eq. 5.2 predicts the photonic strength of the $\lambda_{c}=1300 \mathrm{~nm}$ sample to be $S=9.7 \%$, which is $1.4 \%$ smaller than the measured value. Note that dispersion was not taken into account in Eq. 5.2. The calculated reflectivity curve in Fig. 5.1, which does include dispersion, demonstrated a larger width of $10.9 \pm 0.2 \%$ FWHM, in excellent agreement with our experimental data. 


\subsubsection{Switching experiment}

Following our conclusions in Chapter 3, we optimize the homogeneity of the switched structures, by choosing a pump frequency $\omega_{\text {pump }}=5814 \mathrm{~cm}^{-1}$, just above the two-photon absorption edge of GaAs $\left(\omega_{\text {gap }}=5747 \mathrm{~cm}^{-1}\right.$ [96]). At this pump frequency $\omega_{\text {pump }}=5814 \mathrm{~cm}^{-1}$, the two-photon absorption coefficient $\beta=1.5 \pm 0.5$ $\mathrm{cmGW}^{-1}$ was measured from an open aperture z-scan measurement [114] on a bulk GaAs wafer (see Appendix C). Since the pump frequency is well below the photonic stop gap of our structures, the pump light is not affected by Bragg diffraction.

In all switching experiments presented in this Chapter, pump and probe were cross polarized, and focussed collinearly onto the sample surface by a $f=100$ $\mathrm{mm}$ lens. The resulting probe focus was $w_{\text {probe }}=24 \pm 5 \mu \mathrm{m}$ FWHM, the larger probe focus was $w_{\text {pump }}=42 \pm 5 \mu \mathrm{m}$ FWHM, ensuring lateral homogeneity. The excitation irradiance $I_{0}$ in our experiments was varied from 0 to $500 \mathrm{GWcm}^{-2}$, remaining below the damage threshold of our samples $I_{\text {damage }} \approx 600 \pm 70 \mathrm{GWcm}^{-2}$. For pump irradiance above the damage threshold irreversible changes appear in the reflectivity spectra, indicating structural damage.

\subsection{Ultrafast switching of the photonic strength}

\subsubsection{Differential reflectivity spectra}

The surface plot in Fig. 5.2 illustrates the evolution of the differential reflectivity in the stop band of the $\lambda_{c}=1400 \mathrm{~nm}$ sample, for delay times between -2 ps and +4 ps after excitation. Near $\tau=0$, large, ultrafast changes in the reflectivity take place within 500 fs. At $\tau=2$ ps, the differential reflectivity reached its maximum value, and starts a slow decays at timescales of tens of ps. At $\tau=4 \mathrm{ps}$, increases of up to $\Delta R / R=3.7 \%$ at the blue edge of the stop band, as well as strong decreases of up to $\Delta R / R=-30 \%$ on the red edge of the stop band are observed. At frequencies above the stop band, strong dispersive shapes appear.

\subsubsection{Switched reflectivity spectra}

The shape of the stop band of the $\lambda_{c}=1400 \mathrm{~nm}$ sample and the $\lambda_{c}=1300 \mathrm{~nm}$ sample were probed at a fixed probe delay $\tau=4$ ps, after the initial ultrafast transient effects. Fig. 5.3 shows switched and unswitched reflectivity spectra of the first order stop bands. The absolute scaling error in the resulting reflectivity spectra turned out to be $\Delta R=6 \%$. The data in the reflectivity spectra shown in Fig. 5.3 was scaled by a factor of 1.06 , such that the peak reflectivity coincides with a peak reflectivity of 


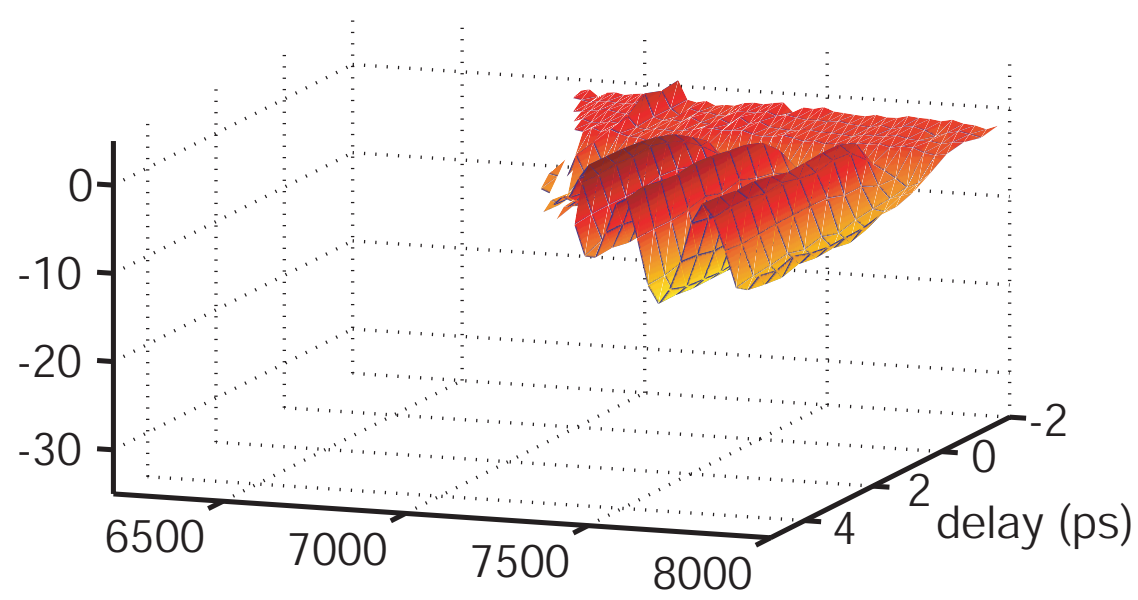

Figure 5.2: Differential reflectivity as a function of both probe frequency and probe delay $\tau$ for the $\lambda_{c}=1400 \mathrm{~nm}$ sample. The pump peak irradiance was $I_{0}=310 \pm 1 \mathrm{GWcm}^{-2} . \tau$ was varied in steps of $\Delta \tau=500 \mathrm{fs}$. The probe wavelength was tuned from 1255 to $1600 \mathrm{~nm}$ in $\Delta \lambda=5 \mathrm{~nm}$ steps. Reflectivity increases of up to $\Delta R / R=3.7 \%$ at the blue edge of the stop band, as well as strong decreases of up to $\Delta R / R=-30 \%$ on the red edge of the stop band are observed. At $2 p s$ after excitation, the induced effect is stable.

$100 \pm 2 \%$, obtained from separate linear reflectivity measurements. The unswitched spectra show broad stop bands $\Delta \omega / \omega_{c}=11 \%$. The bandwidth $(\Delta \omega / \omega)=1.44 \pm 0.05 \%$ of our OPAs causes the unswitched spectra to appear rounded compared to the FT-IR spectrum in Fig. 5.1. The convolution with the OPA linewidth causes the stop band to appear somewhat broader than the actual stop gap. At frequencies below the stop band, we observe the Fabry-Pérot fringes, whose shift caused the strong dispersive shaped in the differential reflectivity in Fig. 5.2. Note that the suppression of the modulation of the Fabry-Pérot fringes compared to the FTS spectrum in Fig. 5.1 is also caused by averaging over the OPA linewidth.

In the switched spectra in Fig. 5.3, the pump irradiance is varied from 0 to $330 \pm 30 \mathrm{GWcm}^{-2}$. The absolute reflectivity measurements on the $\lambda_{c}=1400 \mathrm{~nm}$ sample (Fig. 5.3A, symbols) at a typical pump irradiance $I_{0}=190 \pm 20 \mathrm{GWcm}^{-2}$, show both a large absolute reflectivity decrease from $93 \%$ to $63 \%$ on the red edge of the stop band at $\omega_{\text {red }}=6755 \mathrm{~cm}^{-1}$, as well as a large increase from $65 \%$ to $74 \%$ on the blue edge of the stop band at $\omega_{\text {blue }}=7405 \mathrm{~cm}^{-1}$. The peak reflectivity is not affected by the switching, indicating that the induced absorption remains low. The switched 


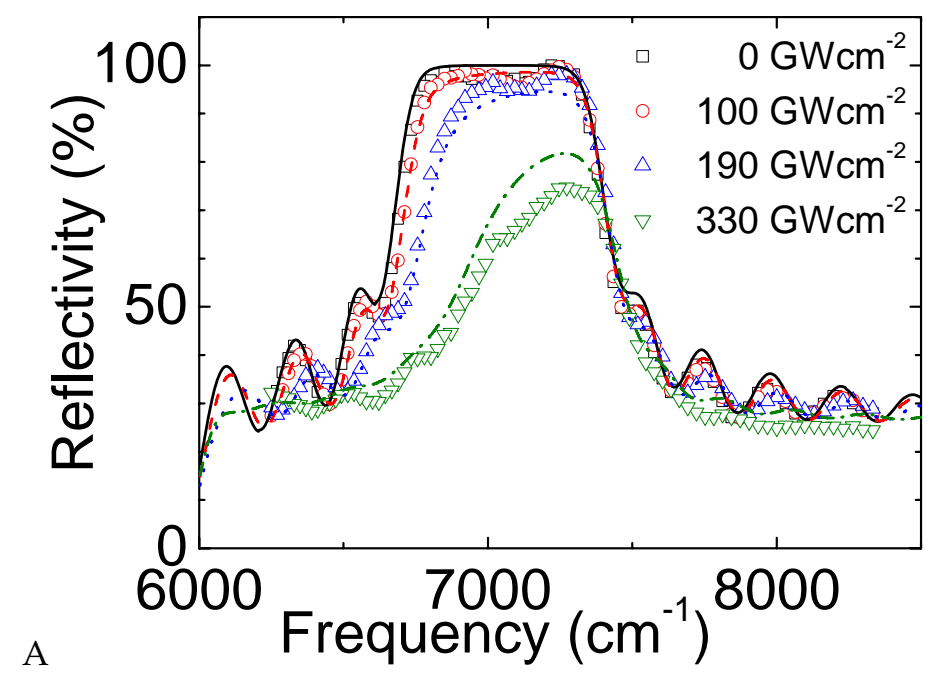

A

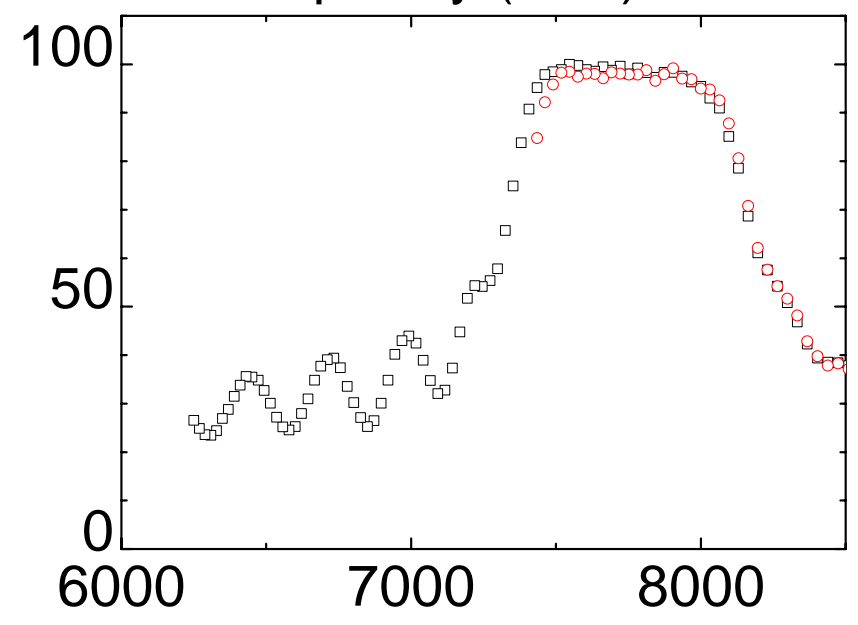

B

Figure 5.3: Ultrafast switching of the first order stop band reflectivity of large GaAs-AlAs multilayer structures (25 pairs). A $\lambda_{c}=1400 \mathrm{~nm}$ sample. The pump irradiance is varied from 0 to $330 \pm 30$ $\mathrm{GWcm}^{-2}$. B $\lambda_{c}=1300 \mathrm{~nm}$ sample. The pump irradiance is varied from $105 \pm 10$ to $290 \pm 30 \mathrm{GWcm}^{-2}$. The absolute reflectivity measurements (symbols) shows both large absolute reflectivity decreases up to $\Delta R>40 \%$ on the red edge of the stop band as well as large increases of up to $\Delta R>8 \%$ on the blue edge of the stop band. Switched spectra were measured at a fixed probe delay $\tau=4 p$ s. The curves are transfer matrix calculations that take into account both the nonlinear absorption of pump light, as well as the Drude model for the GaAs layers (see Section 5.4.1). 
reflectivity measurements reproduce well on the $\lambda_{c}=1300 \mathrm{~nm}$ sample (Fig. 5.3B, symbols) at the same pump irradiance $I_{0}=190 \pm 20 \mathrm{GWcm}^{-2}$. Fig. 5.3B also displays a large decrease from $95 \%$ to $70 \%$ on the red edge of the stop band at $\omega_{\text {red }}=7435$ $\mathrm{cm}^{-1}$, as well as a large increase from $69 \%$ to $76 \%$ up on the blue edge of the stop band at $\omega_{\text {blue }}=8165 \mathrm{~cm}^{-1}$. Here, the maximum reflectivity also remains unchanged.

In both Fig. 5.3A and B, Fabry-Pérot fringes remain visible, even at a high pump irradiance $I_{0}=330 \pm 30 \mathrm{GWcm}^{-2}$, albeit with a reduced modulation depth. This indicates that large reflectivity changes can be achieved in the samples while keeping the induced absorption low, and thus keeping the switched structure transparent. The decrease of the maximum reflectivity with increasing pump irradiance is displayed in Fig. 5.4. We observe that even for a pump irradiance up to $I_{0}=300 \pm 30 \mathrm{GWcm}^{-2}$, the peak reflectivity remains larger than $90 \%$. For even larger pump irradiance, the peak reflectivity rapidly decreases with $I_{0}$, indicating that the probe absorption length in the structure becomes smaller than $L_{B}$, disturbing the build-up of the Bragg reflection peak.

In both samples, strong dispersive shapes in $\Delta R / R$ appear for frequencies outside the stop band. We attribute these periodic variations to the shift of the FabryPérot fringes. The blue shift in the fringes is in good agreement with the reduced effective refractive index $n_{e f f}$, and hence the decreased optical thickness of the photonic structure.

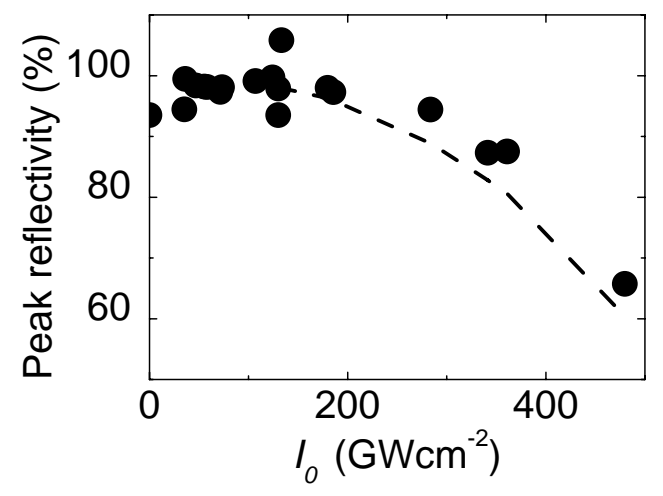

Figure 5.4: Peak reflectivity of the $\lambda_{c}=1300 \mathrm{~nm}$ sample with a center stop band frequency $\omega_{c}=$ $7751 \mathrm{~cm}^{-1}\left(\lambda_{c}=1290 \mathrm{~nm}\right)$ versus pump irradiance. The pump irradiance is varied from 0 to $290 \pm 30$ $\mathrm{GWcm}^{-2}$. The maximum reflectivity remains larger than $R_{\max }=95 \%$ for pump irradiance $\mathrm{I}_{0}=300 \pm 30$ $\mathrm{GWCm}^{-2}$, indicating the induced absorption remains small. The curves are peak reflectivities obtained from transfer matrix calculations (see Section 5.4). 


\subsubsection{Irradiance scaling of the photonic strength}

To quantify the observed changes in the photonic strength of the structures, the relative stop band widths $\Delta \omega / \omega_{c}$ at full width of half maximum (FWHM) were measured at various pump irradiance. In Fig. 5.5A and B, the stop band widths are plotted versus pump irradiance (symbols). We observe a large decrease of the stop band width from $10.9 \pm 0.2 \%$ to $9.5 \pm 0.2 \%$ for the $\lambda_{c}=1400 \mathrm{~nm}$ sample (A), and from $11.0 \pm 0.2 \%$ to $9.5 \pm 0.2 \%$ for the $\lambda_{c}=1300 \mathrm{~nm}$ sample (B) for pump irradiance of about $300 \mathrm{GWcm}^{-2}$. This indicates a strongly reduced photonic strength $S$ of the structures, caused by the reduced refractive index contrast. The curves are TM model calculations that are in good agreement with the experimental data (see Section 5.4). The observations are consistent with a reduced dielectric contrast $\Delta \epsilon$, which reduces the photonic strength $S$, causing the stop band to become narrower.
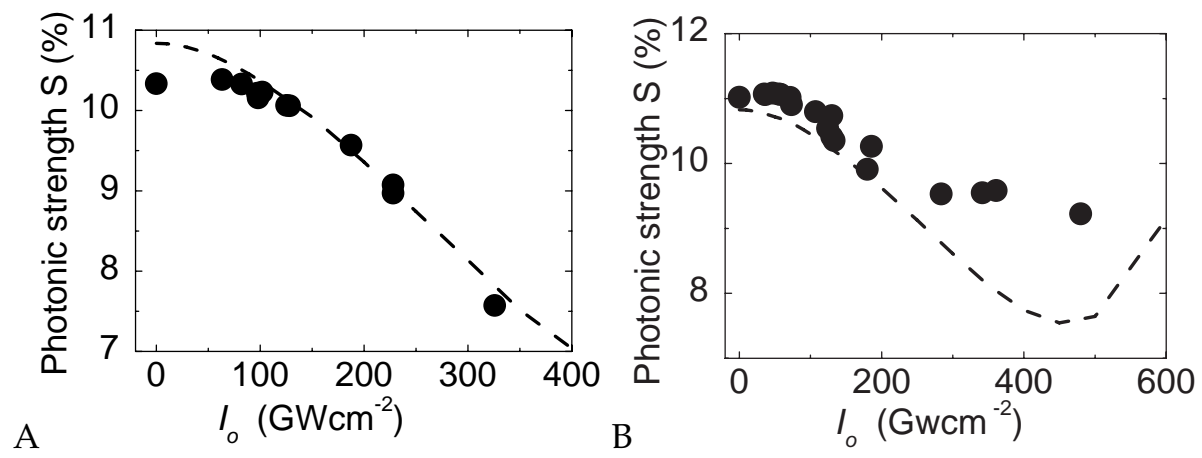

Figure 5.5: The stop band width (left hand ordinate) and the corresponding photonic strength $S$ (right hand ordinate) for the $\lambda_{c}=1400 \mathrm{~nm}$ sample (A) and the $\lambda_{c}=1300 \mathrm{~nm}$ sample (B) as a function of pump irradiance. The data were obtained from both the measured spectra (symbols) as well as from the modeled spectra (curves) and are plotted versus pump irradiance. The observed large decrease of the stop band width with increasing pump irradiance, indicates a strongly reduced photonic strength $S$ of the structure, caused by the reduced refractive index contrast. We find good agreement for pump irradiance up to $300 \pm 30 \mathrm{GWcm}^{-2}$.

We have also measured the change in the center position $\omega_{c}$ of the stop band in both samples. Figures $5.6 \mathrm{~A}$ and $\mathrm{B}$, show $\omega_{c}$ as a function of pump irradiance (symbols). We observe that in the $\lambda_{c}=1400 \mathrm{~nm}$ sample (A), for pump irradiance $I_{0}=330 \pm 30 \mathrm{GWcm}^{-2}, \omega_{c}$ increases by as much as $\Delta \omega_{c}=150 \pm 15 \mathrm{~cm}^{-1}$, or $\Delta \omega_{c} / \omega_{c}=$ $2.1 \pm 0.2 \%$. The center frequency of the $\lambda_{c}=1300 \mathrm{~nm}$ sample (B), for pump irradiance $I_{0}=300 \pm 30 \mathrm{GWcm}^{-2}$ shifts by $\Delta \omega_{c}=115 \pm 10 \mathrm{~cm}^{-1}$, or $\Delta \omega_{\mathcal{c}} / \omega_{c}=1.5 \pm 0.2 \%$. The large shift of the center position towards higher frequency with increasing pump 


\subsubsection{Temporal evolution of the reflectivity}

High resolution time resolved reflectivity was measured on the $\lambda_{c}=1400 \mathrm{~nm}$ sample to investigate the dynamics of the induced change in photonic strength. The dispersion in the probe path (200 fs) in zero delay position for each probe frequency was manually adjusted. The absolute reflectivity change $\Delta \mathrm{R}$ at salient frequencies is plotted versus probe delay in Fig. 5.7. The pump conditions are the same as in Fig. 5.3, the pump irradiance is $I_{0}=180 \pm 20 \mathrm{GWcm}^{-2}$.

At the red edge of the gap : $\omega_{\text {probe }}=6711 \mathrm{~cm}^{-1}(\lambda=1.5 \mu \mathrm{m})$, the absolute reflectivity decreases by as much as $-53 \pm 1 \%$ (filled squares). The switching time is taken as the time in which the reflectivity change increases from $10 \%$ to $90 \%$ of the maximum change. On the red edge this corresponds to a reflectivity change of $-7 \%$ and $-47 \%$ respectively. From Fig. 5.7 we obtain a switching time $\Delta t_{r e d}=$ $200 \pm 20 \mathrm{fs}$, of the order of the pump autocorrelation FWHM, see Fig. 4.6 (lower panel). This observation confirms that change in the reflectivity happens almost instantaneously.

At the blue edge of the gap: $\omega_{\text {probe }}=7407 \mathrm{~cm}^{-1}, \Delta \mathrm{R}_{\text {blue }}$ first decreases to $-5.4 \pm 0.2 \%$ within $\Delta \mathrm{t}=290 \pm 30 \mathrm{fs}$, and then increases to attain a positive value of $+2.2 \pm 0.2 \%$ within $\Delta t_{\text {blue }}=360 \pm 30$ fs. The initial decrease in reflectivity at the blue edge is attributed to a Kerr nonlinearity in the AlAs layers, as it is only observed on the high frequency edge of the stop band. Similar non monotonic effects were observed on the blue edge of the stop band of Si woodpile photonic crystals discussed in Chapter 6.

On both samples, the transient behavior of the absolute reflectivity change at longer delay times was studied for probe frequencies on the red edge of the stop band (see Fig. 5.8). In the $\lambda_{c}=1400 \mathrm{~nm}$ sample (A), the maximum decrease at the red edge at $\omega_{\text {red }}=6711 \mathrm{~cm}^{-1}$ is $\Delta \mathrm{R}=-57 \pm 2 \%$. The transient reflectivity at longer times after excitation is well fitted by a double exponential decay:

$$
\Delta R(\tau)=A_{1} e^{-\tau / t_{1}}+A_{2} e^{-\tau / t_{2}}
$$

where optimum parameters for the $\lambda_{c}=1400 \mathrm{~nm}$ sample were found to be $A_{1}=30 \%$, $t_{1}=36 \mathrm{ps} A_{2}=34 \% t_{2}=896 \mathrm{ps}$, resulting in a fit with $\chi^{2}=0.74$. For the $\lambda_{c}=1300$ nm sample (B), we find $A_{1}=33 \%, t_{1}=26$ ps $A_{2}=27 \% t_{2}=703$ ps, resulting in a fit with $\chi^{2}=0.90$. In both samples we observe an initial fast recovery at $30-40 \mathrm{ps}$ timescales, followed by a slow decay in $\sim 1 \mathrm{~ns}$. Our observations suggest that there is an initial carrier cooling processes due to interaction of the electrons with the lattice-phonons, followed by a much slower recombination of the carriers. The resulting combined (1/e) decay time $\tau_{(1 / e)}=0.8 \mathrm{~ns}$, suggests that large absolute 


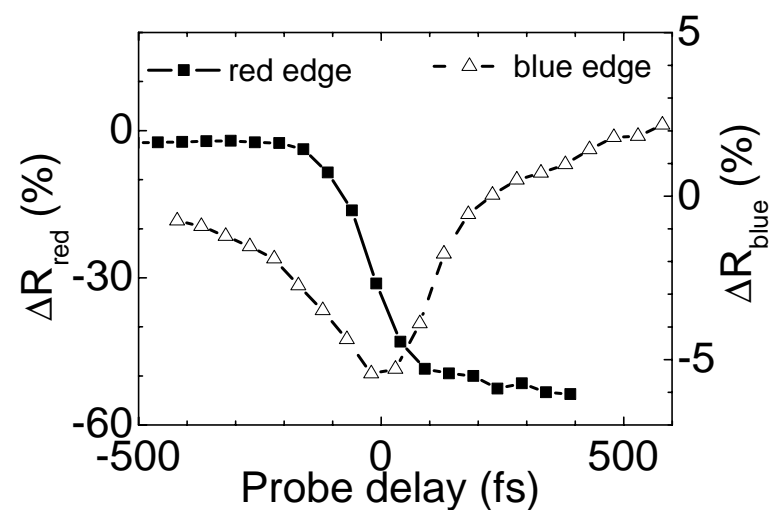

Figure 5.7: High resolution time resolved reflectivity changes at salient frequencies of the $\lambda_{c}=1400$ $n m$ sample. The pump irradiance is $180 \pm 20 \mathrm{GWcm}^{-2}$. At $\omega_{\text {red }}=6711 \mathrm{~cm}^{-1}$ (red edge of the stop band), the reflectivity decreases by as much as $53 \pm 1 \%$ while at $\omega_{\text {blue }}=7407 \mathrm{~cm}^{-1}$ (blue edge of the stop band), the reflectivity first decreases by $-5.4 \pm 0.2 \%$, and then increases again to $+2.2 \pm 0.2 \%$. We deduce a switching time of $200 \pm 20 \mathrm{fs}$, confirming a large and ultrafast reflectivity change.

reflectivity changes of $\Delta R=-53 \pm 2 \%$ are feasible at switching bandwidths of up to 1 $\mathrm{GHz}$, which is relevant for potential future applications.

A
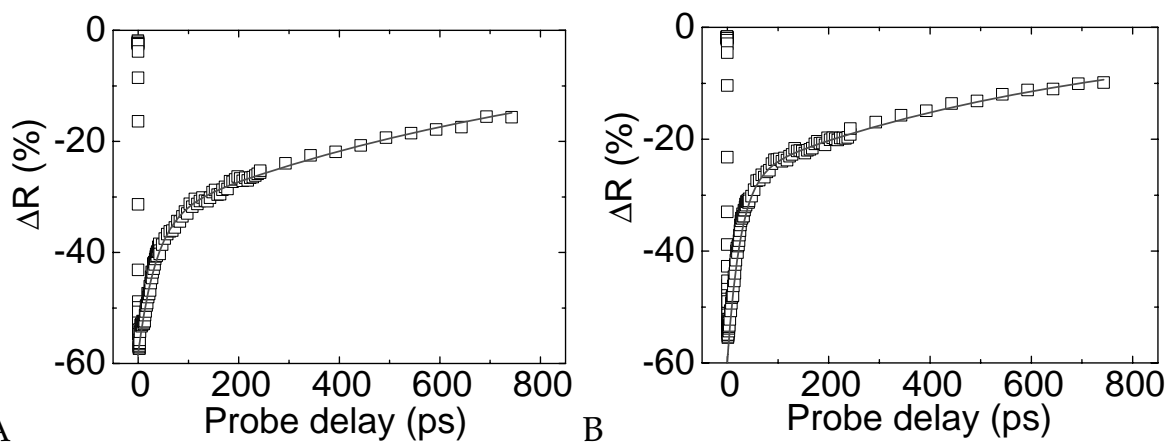

Figure 5.8: Time resolved reflectivity changes at the low frequency edge of the $\lambda_{c}=1400 \mathrm{~nm}$ sample (A) and the $\lambda_{c}=1300 \mathrm{~nm}$ sample (B)). Pump conditions are the same as in Fig. 5.3, the pump irradiance is $180 \pm 20 \mathrm{GWcm}^{-2}$. The curves are fitted double exponential fits to the data. We find decay times $t_{1}=36 \pm 3 \mathrm{ps}$ and $t_{2}=0.9 \pm 0.1 \mathrm{~ns}$ for the $\lambda_{c}=1400 \mathrm{~nm}$ sample, and $t_{1}=26 \pm 3 \mathrm{ps}$ and $t_{2}=$ $0.7 \pm 0.1 \mathrm{~ns}$ for the $\lambda_{c}=1300 \mathrm{~nm}$ sample. 


\subsection{Theoretical model of switched DBR samples}

\subsubsection{Transfer matrix theory}

A quantitative analysis of the measured spectra was based on a $2 \times 2$ transfer matrix model (see for instance Ref. [115], p.70). This model was expanded by including several important parameters. First of all, the refractive index dispersion for both GaAs and AlAs were taken from Ref. [116] and included in the model. Secondly, the spatial homogeneity of the switched samples was taken into account. We have used Eq. 3.4 to obtain the nonlinear pump irradiance in the structure [117]. The two-photon absorption coefficient $\beta$ is one of the three free parameters in our model. The generated carrier density $N_{e h}(z)$ for each GaAs layer located at depth $z$ is obtained from the distribution of pump light $I(z)$ via Eq. 3.5.

The induced refractive index change in the switched GaAs layers was obtained from the Drude model (see Appendix A). The induced absorption is related to the momentum scattering time. We have corrected the momentum scattering time for electron-electron and electron-hole scattering which dominate the momentum relaxation at the relatively high carrier densities in our experiments $\approx 10^{19} \mathrm{~cm}^{-3}$ (see Eq. A.3) [118]. We obtain a momentum scattering time of order of $20 \mathrm{fs}$. We have also taken into account the effect of interband absorption [119], where the interband absorption cross-section $\sigma_{\text {intra }}$ is used as the second free parameter in our model, since it is unknown at the carrier densities in our experiments (see Eq. A.11).

To compensate for the fact that our experimental data are averaged over the linewidth of our OPAs, a convolution with a modeled Gaussian peak with a width $(\Delta \omega / \omega)=1.44 \pm 0.05 \%$ was carried out for each frequency point of the calculated spectra. Including all above features in our model, allows a direct comparison between the modeled spectra and the spectra measured with our setup.

\subsubsection{Optimization procedure}

To demonstrate the optimization procedure that was followed, we consider the switching experiment shown in Fig. 5.3A at a pump irradiance $I_{0}=330 \pm 30 \mathrm{GWcm}^{-2}$. We first set $\sigma_{\text {interband }}$ to zero to exclude the effect of induced absorption.

The first free parameter of the model; $\omega_{c}$ is deduced by comparing calculated spectra to unswitched FTS spectra (see Fig. 5.1). We find $\omega_{c}=7042 \mathrm{~cm}^{-1}$ for the $\lambda_{c}=1400 \mathrm{~nm}$ sample and $\omega_{c}=7737 \mathrm{~cm}^{-1}$ for the $\lambda_{c}=1300 \mathrm{~nm}$ sample.

The second free parameter; $\beta$ is adjusted until the calculated Fabry-Pérot fringes above and below the stop band best fit the measured fringes at the various pump irradiance, for both samples. We find good agreement for a value of $\beta=0.89 \pm 0.05$ 
$\mathrm{cmGW}^{-1}$, which is slightly smaller than the value $\beta=1.5 \pm 0.5 \mathrm{cmGW}^{-1}$ obtained for bulk GaAs (see Appendix C). From the deduced $\beta$, we derive an induced carrier density of $N_{e h}=2.9 \times 10^{19} \mathrm{~cm}^{-3}$ in the first layer, corresponding to a refractive index change in the GaAs layers of $\Delta n_{G a A s}^{\prime}(\omega) / n_{G a A s}^{\prime}(\omega)=2.9 \%$. The large refractive index change only decreases to a value of $2.6 \%$ in the 25 th layer, demonstrating excellent spatial homogeneity.

The third free parameter; $\sigma_{\text {interband }}$ is adjusted until both the calculated decrease in peak reflectivity and the reduction in modulation depth of the Fabry-Pérot fringes fit the measured data in the whole range of pump irradiance for both samples. We find best agreement for an interband absorption crossection $\sigma_{\text {interband }}=2.2 \times 10^{-20} \mathrm{~m}^{2}$, which is about 40 times larger than the cross-section $\sigma_{\text {interband }}=5.6 \times 10^{-22} \mathrm{~m}^{2}$ found by Ref. [119] at about 10 times lower carrier densities.

\subsubsection{Spectral comparison between measurement and theory}

After finding the optimal values for $\beta$ and $\sigma_{\text {inter }}$, we compare the calculated reflectivity spectra for a range of pump irradiance from 0 to $330 \mathrm{GWcm}^{-2}$ to our measured spectra. The modeled reflectivity spectra at various pump irradiance are shown as curves in Fig. 5.3A and B, and compare well to the measured data for both samples. The good agreement between modeled and calculated spectra in Fig. 5.3A and B is striking, since our model contains only three experimentally constraint fitting parameters: $\omega_{c}, \beta$ and $\sigma_{\text {inter }}$.

\subsubsection{Induced absorption}

The good agreement with the measured data is further emphasized by the similarity between the maximum reflectivity of the model and the measurements at different pump irradiance, shown in Fig. 5.4. Our model predicts the maximum reflectivity within $5 \%$ for pump irradiance up to $I_{0}=450 \pm 50 \mathrm{GWcm}^{-2}$. Even at a large pump irradiance of $190 \pm 20 \mathrm{GW} \mathrm{cm}^{-2}$ (dotted curve in Fig. 5.3A), the corresponding induced absorption in the GaAs layers at $\omega_{c}=7042 \mathrm{~cm}^{-1}$ remains limited to only $n_{G a A s}^{\prime \prime}\left(\omega_{c}\right)=$ 0.026. The corresponding probe absorption length is $\ell_{a b s}=\lambda / 4 \pi n_{G a A s}^{\prime \prime}\left(\omega_{c}\right)=4.3 \mu \mathrm{m}$ or $41 \mathrm{GaAs}$ layers, leading to the important conclusion that the structure remains transparent even after switching with a dense carrier plasma.

\subsubsection{Changes in photonic strength and center frequency}

Finally, we used our model to study two characteristic photonic properties of our samples. Firstly, the photonic strength $S$ of the structures is plotted as a function 
of pump irradiance $I_{0}$ in Fig. 5.5A and B. The photonic strengths deduced from the corresponding modeled spectra were plotted as dashed curves in the same Figure. For both samples, our model predicts the photonic strength within $0.5 \%$ for pump irradiance up to $300 \pm 30 \mathrm{GWcm}^{-2}$. Only at very high irradiance, the FWHM of the calculated spectra deviates from the measured value, particularly in the $\lambda_{c}=1300$ nm sample (see Fig. 5.5B).

Secondly, we also study the shift of the center frequency $\omega_{c}$ in both samples as a function of pump irradiance. Both experimental results (symbols) and data obtained from our model (curves), are shown as in Fig. 5.6A and B. Our model is found to give a good prediction of the center frequency of the stop band for pump irradiance up to $300 \pm 30 \mathrm{GWcm}^{-2}$. In the $\lambda_{c}=1300 \mathrm{~nm}$ sample, at very high irradiance, exceeding $I_{0}=300 \pm 30 \mathrm{GWcm}^{-2}$, the model for deviates from the measured data.

We propose two possible explanations for the differences at very high pump irradiance. First of all, additional nonlinear effects in the AlAs layers should also be taken into account. In our model, any changes to the AlAs layers have been left out. Three photon absorption processes in the AlAs layers may no longer be negligible at pump irradiance above. Secondly, the assumption that the interband absorption crossection $\sigma_{\text {inter }}$ is constant with carrier density may not be correct at our large carrier densities.

\subsection{Conclusions and outlook}

\subsubsection{Conclusions}

We have used optical free-carrier generation by ultrashort laser pulses to switch the photonic strength of of 25-pair thick GaAs-AlAs photonic structures. The penetration depth of pump light was maximized by choosing two-photon process to excite free carriers in the GaAs layers. The resulting absolute reflectivity changes in the stop band were studied as a function of both probe frequency and delay time. We have observed large and ultrafast reflectivity decreases of up to $\Delta R>40 \%$ on the red edge of the stop band, as well as large increases of up to $\Delta R>8 \%$ on the blue edge of the stop band. High resolution time resolved measurements demonstrated ultrafast switching timescales of typically $200 \mathrm{fs}$. On ultrafast timescales below $300 \mathrm{fs}$, we observe non-monotonic behavior of the reflectivity at the high frequency edge of the stop band: a rapid decrease within $300 \mathrm{fs}$ due to a Kerr effect, is followed by an increase in the reflectivity due to free carriers.

Essential photonic properties of the stop band were studied as a function of 
pump irradiance. Stop band widths and center positions were plotted versus pump irradiance. We observe both a narrowing of the stop gap, which is caused by the reduced photonic strength of the structure, as well as a blue shift in the center position of the band, which is related to a reduced average refractive index.

The measured spectra were compared to transfer matrix theory. Our model takes into account the nonlinear absorption of pump light, the bandwidth of our laser system. The refractive index of the excited GaAs layers is described by a Drude model that includes electron-electron as well as electron-phonon momentum relaxation processes. We also include the effect of interband absorption. Our model uses three experimentally constraint fitting parameters which are found to be: $\beta=$ $0.89 \mathrm{cmGW}^{-1}, \sigma_{\text {inter }}=2.2 \times 10^{-20} \mathrm{~m}^{2}$ and peak position $\omega_{c}=7040 \mathrm{~cm}^{-1}\left(\lambda_{c}=1400 \mathrm{~nm}\right.$ sample) or $\omega_{c}=7750 \mathrm{~cm}^{-1}$ (the $\lambda_{c}=1300 \mathrm{~nm}$ sample).

From the comparison of the data to our model, we conclude that the change in refractive index of the GaAs layers is large and homogeneous throughout the sample, while the switched structure remains transparent. We deduce a large change in the refractive index of up to $\Delta n_{G a A s}^{\prime}(\omega) / n_{G a A s}^{\prime}(\omega)=2.9 \%$ while maintaining a large (1/e) pump absorption length of $30 \mathrm{GaAs}$ layers, indicating excellent spatial homogeneity. The (1/e) absorption length for probe light remains more than 11 unit cells. Since the induced absorption scales with carrier density, it can be further reduced by reducing the pump irradiance, at the cost of a smaller effect in $\Delta n_{G a A s}^{\prime}(\omega)$. We find good agreement between model and measurement for the maximum reflectivity, the photonic strength, and the center position of the stop band up to a pump irradiance of $I_{0}=300 \pm 30 \mathrm{GWcm}^{-2}$.

\subsubsection{Outlook}

The results and interpretation presented here demonstrate how two-photon absorption can be utilize to homogeneously excite freecarriers in a semiconductor photonic structure. We have interpreted the resulting ultrafast changes in photonic properties of the structure, namely, the photonic strength and the position of the stop band. Consequently, our results are important for the interpretation of switching experiments in three-dimensional photonic crystal that can posses a photonic band gap, such as Si woodpile photonic crystals described in Chapter 6 or $\mathrm{Si}$ inverse opals in the second order stop band region (see Chapter 7). Such crystals have a sufficiently large photonic strength for stop bands to appear in all directions simultaneously, which would result in a photonic band gap. By switching the photonic strength in these structures, large variations in the DOS are expected to take place. 


\section{Chapter}

\section{Ultrafast switching of Si woodpile photonic band gap crystals}

We present the first ultrafast all-optical switching measurements of photonic band gap crystals. The Si woodpile crystals are homogeneously excited, and probed by measuring reflectivity over an octave in frequency (including the telecom range) as a function of time. At short delay times $<200 \mathrm{fs}$, we observe that the photonic gap becomes unexpectedly narrower than in the unswitched case. After 1 ps, the complete gap has shifted to higher frequencies. This intricate behavior is the result of competing refractive index changes due to an electronic Kerr nonlinearity and to optically excited free carriers. The frequency shift of the band gap as a function of pump irradiance agrees well with exact modal method calculations. We briefly discuss possible applications.

\subsection{Introduction}

Exciting prospects will arise if photonic band gap crystals are switched on ultrafast timescales. In the first place, switching a photonic band gap crystal provides dynamic control over the density of states that would allow the switching-on or -off of light sources [54]. Secondly, switching would allow the controlled capture or release of photons from photonic band gap cavities [54], which is relevant to solid-state slow-light schemes [64]. Thirdly, switching the directional properties of 
photonic crystals leads to fast changes in the reflectivity, where interesting changes have been reported for Bragg stacks [66, 67], 2D photonic crystals [68, 69], and first-order stop bands of 3D opaline crystals [90,91]. Finally, ultrafast control of the propagation of light is essential to future applications in active photonic integrated circuits [120].

It is well-known that semiconductors have favorable properties for optical switching, hence they are excellent constituents for switchable photonic materials [121, 122]. Moreover, their elevated refractive indices are highly advantageous to photonic crystals per se $[22,25,33,34,105,123]$. Therefore, we present in this Chapter ultrafast all-optical switching experiments on Si woodpile photonic band gap crystals.

\subsection{Si woodpile photonic band gap crystals}

The Si woodpile photonic crystals are made using a layer-by-layer approach that allows convenient tuning of the operating wavelengths; here the crystals are designed to have a photonic band gap around the telecommunication wavelength of $1.55 \mu \mathrm{m}[22,25]$. We had to our disposition two Si woodpile crystals made by Dr. Jim Fleming from Sandia and characterized by the Polman group at AMOLF [124]. The samples, called WP1 and WP2, had dimensions of about $14 \times 8 \mathrm{~mm}^{2}$. The crystals consist of five layers of stacked polycrystalline $\mathrm{Si}$ nanorods that have a refractive index of 3.45 at $1.55 \mu \mathrm{m}$ [96]. Both samples are subdivided in squares of $2 \times 2 \mathrm{~mm}^{2}$, as shown in Fig. 6.1. On inspection by eye, the samples demonstrate strong grating behavior for visible light, since the color changes with viewing angle. Optically, the samples are almost free of surface defects. Only a small $<1 \%$ part of the crystal's surface optically shows damage, notably small scratches that have occurred after the fabrication of the sample.

From each individual square on the samples, scanning electron micrograph (SEM) images were made at a range of magnifications. We investigate both the surface quality of the sample as well as the lateral alignment of the layers in the structure with respect to each other (see Fig. 6.2). Low magnification SEM images were used to verify that the crystal's lattice parameter is constant within each square. The crystal is subdivided in square domains of $2 \times 2 \mathrm{~mm}^{2}$ that are large compares to domains in opal-type photonic crystals that typically $\approx 20-30 \mu \mathrm{m}$ in size (see Chapter 7). The lateral alignment of the layers in the crystals varies systematically from square to square, see Fig. 6.2. The variation ensures that in at least 1 of 2 squares on the sample the layers are properly aligned. Our experiments 


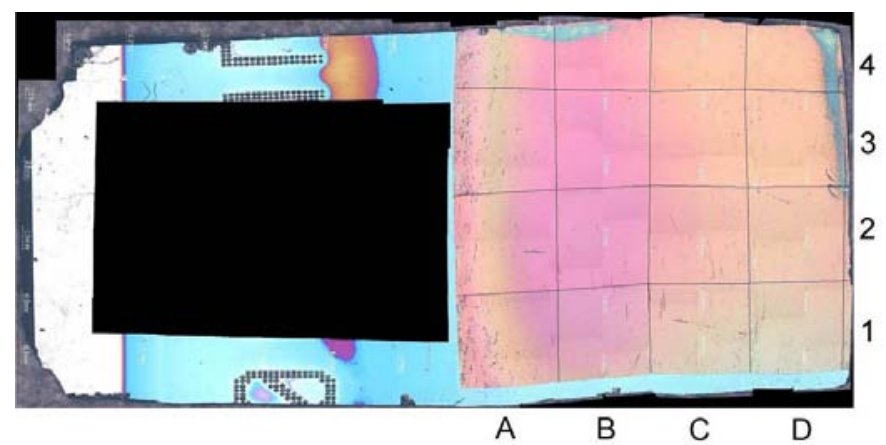

Figure 6.1: (color) Composite top view of sample WP1 from images taken with an optical microscope. The five-layer thick woodpile crystals are fabricated on the right-hand part of the sample, and is subdivided in $16 \approx 2 \times 2 \mathrm{~mm}^{2}$ squares. Crystal boundaries are marked by a black curve, small deviations from a straight line are caused by the image stitching process. Only a small $<1 \%$ part of the crystal's surface shows optical damage. In particular square C3, which was used in most of our measurements, is almost free of surface defects.

have focused on the well-aligned squares C3 and D4 of sample WP1. The surface quality in both samples WP1 and WP2 turns out to be excellent; no fabrication defects or point defects were observed in the SEM images.

SEM images such as the ones shown in Fig. 6.2 for sample WP1, were used to measure the dimensions of both samples, such as the rod dimensions, as well as the random variations in the structure. Figure 6.3 summarizes the structural data obtained. Since all switching measurements presented in this Chapter were done on either square C3 or square D4 of sample WP1, the remainder of this Section will focus on sample WP1. In sample WP1 the rod width is $175 \pm 5 \mathrm{~nm}$, and the rod spacing in each layer alternates between 600 and $700 \pm 10 \mathrm{~nm}$. This superstructure results in a doubled unit cell size of $1300 \mathrm{~nm}$. While each second rod in the crystal is slightly displaced by $50 \mathrm{~nm}$, this periodic perturbation and the resulting superstructure do not affect the photonic band gap region [124]. From side view images, such as the inset in Fig. 6.4, the average rod height of sample WP1 was measured to be $\bar{c}=155 \mathrm{~nm}$. The values for the rod thickness $\bar{c}$ and the period $\bar{b}$ show that strictly speaking the unit cell of sample WP1 is orthorhombic instead of face centered cubic. The in-plane lattice parameter of the crystal is $\sqrt{2} \cdot 650 \mathrm{~nm}$ $=919 \mathrm{~nm}$, while the thickness of one unit cell (four layers) is $620 \mathrm{~nm}$. In sample WP2 the rod width is $\mathrm{a}=175 \pm 5 \mathrm{~nm}$, unit cell dimensions are $\sqrt{2} \cdot 600 \mathrm{~nm}=849$ $\mathrm{nm}$ in the lateral directions.

Random scattering from structural variations is an important cause of extinc- 


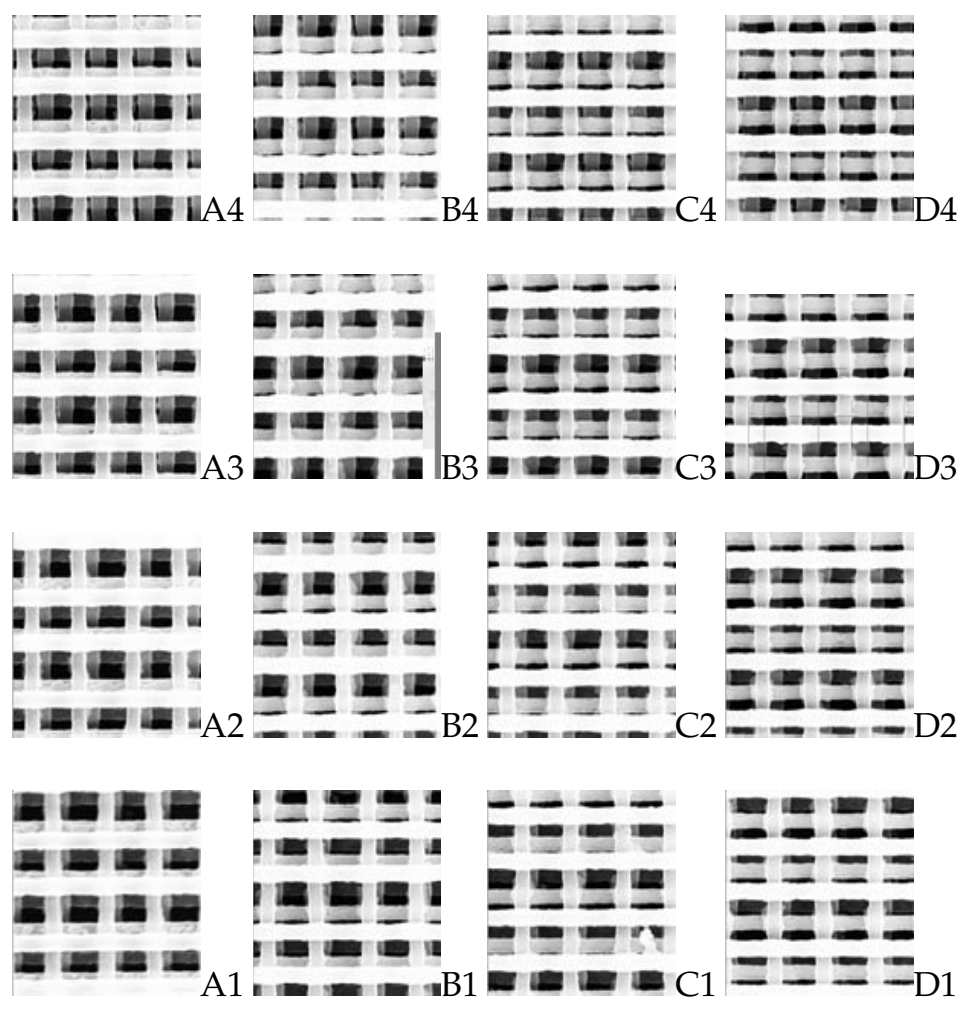

Figure 6.2: Top view SEM images taken from the 16 squares in the WP1 crystal. The coordinates of the squares refer to the positions shown in Fig. 6.1. The contrast is adjusted such that all five layers of the crystal are visible. The systematic variation in the lateral position of each layer ensures that in at least 1 of 2 squares on the sample the layers are aligned properly. Our experiments have focused on the well-aligned squares $C 3$ and D4.

tion of light inside photonic crystals [17]. Extinction has an adverse effect on the photonic properties of a crystal, which we will discuss in more detail in Section 6.15. Therefore, we have quantified the roughness of our crystals from high resolution scanning electron micrographs, see Fig. 6.4. From the top view image of square $\mathrm{C} 3$ of sample WP1, the random variation in rod width $\triangle \mathrm{a} / \mathrm{a}$ was determined to be 5\%. The side view SEM image in the inset in Fig. 6.4 reveals thin sheetlike features that appear in between the layers of rods. The features are remainders of the $\mathrm{SiN}$ layers used in the production process and give rise to additional random scattering that is hard to quantify. Nevertheless, it appears that WP1 is a strongly reflecting sample that is well suited for switching experiments. 

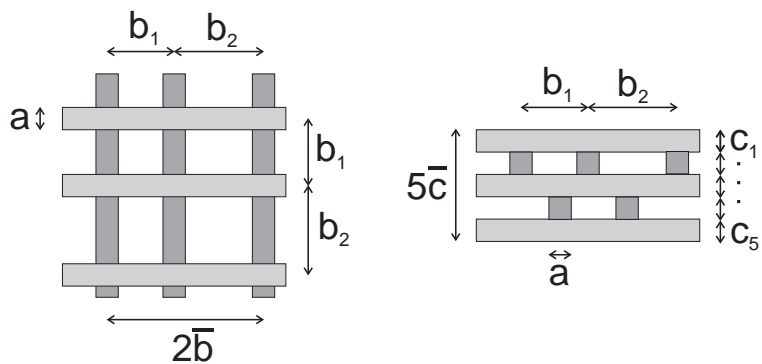

\begin{tabular}{l||l|l|l|l|l} 
& $\mathrm{a}$ & $\mathrm{b}_{1}$ & $\mathrm{~b}_{2}$ & $\overline{\mathrm{b}}$ & $\mathrm{c}_{1}$ \\
\hline WP1 & $175 \pm 5$ & $600 \pm 5$ & $700 \pm 5$ & $650 \pm 5$ & $190 \pm 15$ \\
\hline WP2 & $175 \pm 5$ & $550 \pm 5$ & $650 \pm 5$ & $600 \pm 5$ & - \\
& & & & & \\
& $\mathrm{c}_{2}$ & $\mathrm{c}_{3}$ & $\mathrm{c}_{4}$ & $\mathrm{c}_{5}$ & $\overline{\mathrm{c}}$ \\
\hline WP1 & $126 \pm 15$ & $138 \pm 15$ & $174 \pm 15$ & $152 \pm 15$ & $155 \pm 5$ \\
\hline WP2 & - & - & - & - & $200 \pm 10$
\end{tabular}

Figure 6.3: Schematic drawing of top and side view of the woodpile crystal. The slight difference between $b_{1}$ and $b_{2}$ results in the superstructure of the crystal The table shows the dimensions of both crystal WP1 and WP2 that were obtained from SEM images. The value of $\bar{c}$ for sample WP2 was deduced from reflectivity spectra. All dimensions are in $n m .^{a}$

${ }^{a}$ Ref. [124] has also reported experiments on either sample WP1 or WP2. Surprisingly, the reported sample dimensions $\mathrm{b}_{1}=600 \mathrm{~nm}, \mathrm{~b}_{2}=700 \mathrm{~nm}$, and $\overline{\mathrm{c}}=200 \mathrm{~nm}$, seem to be inconsistent with the dimensions measured by us.

\subsection{Linear reflectivity spectra}

\subsubsection{Stop band at normal incidence}

Linear unpolarized reflectivity measurements at normal incidence along the $\{001\}$ direction of the woodpile lattice were done with a FT-IR reflectivity setup similar to the one described by Ref. [113]. The reflectivity signal was referenced to that of a gold mirror to obtain an absolute reflectivity calibration. Reflectivity spectra were measured on various squares on each sample. Surprisingly, the considerable difference in lateral alignment of the rods (see Fig. 6.2), affects the shape of the stop band by less than $\Delta \mathrm{R}<5 \%$ only. Likely this is because the $\{001\}$ stop band is predominantly related to the periodicity in the $\mathrm{z}$ direction, which is not affected by lateral misalignment.

Broadband reflectivity spectra of well-aligned squares of both samples are 


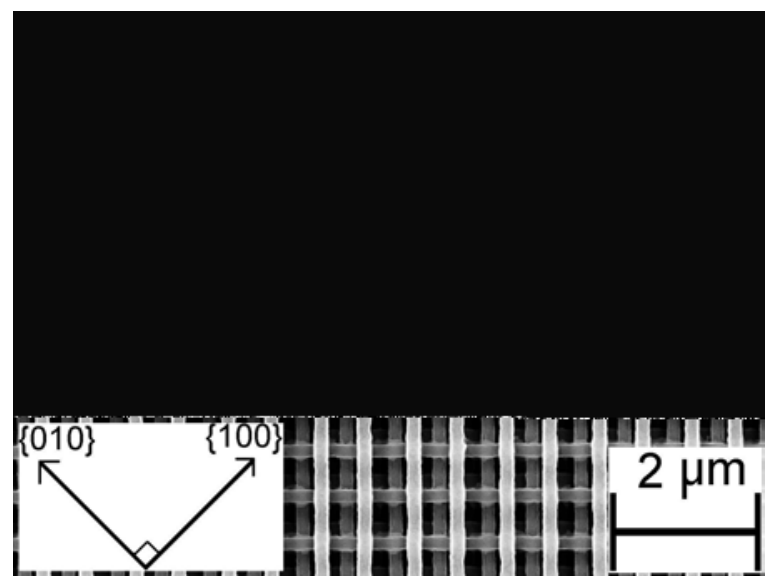

Figure 6.4: High resolution scanning electron micrographs of a $\{001\}$ surface of a Si woodpile crystal. The average lateral distance between two consecutive rods is $650 \pm 10 \mathrm{~nm}$. The arrows indicate the crystal's $\{010\}$ and $\{100\}$ direction. Inset: side view of the crystal. The width and thickness of each rod is $175 \pm 10 \mathrm{~nm}$ and $155 \pm 10 \mathrm{~nm}$ respectively.

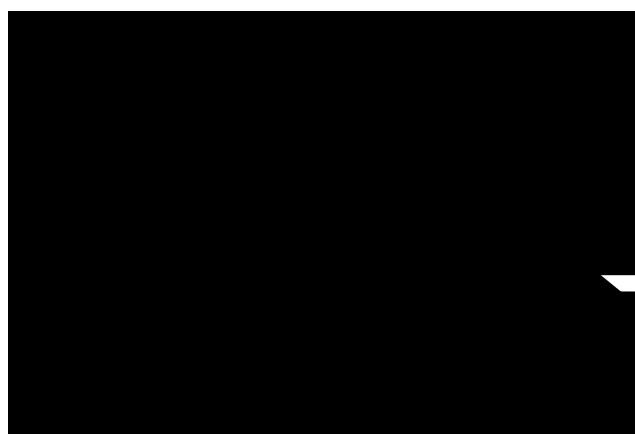

Figure 6.5: (symbols) Linear FT-IR reflectivity measurements for unpolarized light on well-aligned squares on sample WP1 (A) and WP2 (B).(dashed curves) Calculated spectra, assuming a well aligned woodpile structure without superstructure and without dispersion. (A) Sample WP1, using rod height $\bar{c}=155 \mathrm{~nm}$ (obtained from SEM). Both the peak position and width in the measurement agree well with the calculation. (B) Sample WP2, a rod height $\bar{c}=200 \mathrm{~nm}$ was found by comparing the position of the peak in experiment and calculation.

shown in Fig. 6.5A (crystal WP1), and in Fig. 6.5B (crystal WP2). Even though the crystals are relatively thin, the strong photonic interaction strength and the excellent crystal quality result in a high reflectivity of up to $95 \%$, higher than in 
bulk Si and than in Si inverse opal photonic structures (see Chapter 7). The broad stop bands shown in Fig. 6.5 were measured in the $\{001\}$ direction of the woodpile lattice, and correspond to the $\Gamma-\mathrm{X}$ stop gap in the band structure, which is part of the $3 \mathrm{D}$ photonic band gap of $\mathrm{Si}$ woodpile photonic crystals [22, 125]. We determine the full width at half maximum of the stop bands after subtraction of the $\approx 10 \%$ background reflectivity. Crystal WP1 exhibits a broad stop band for frequencies from $5570 \mathrm{~cm}^{-1}$ to $8700 \mathrm{~cm}^{-1}$ ( $\omega_{\text {center }}=7135 \mathrm{~cm}^{-1}, \Delta \omega / \omega=44 \%$ FWHM). The stop band in the second crystal WP2 is considerably red-shifted to $\omega_{\text {center }}=6300 \mathrm{~cm}^{-1}$, and lies between $4900 \mathrm{~cm}^{-1}$ and $7700 \mathrm{~cm}^{-1}$. The resulting width of the stop band of sample WP2 $\Delta \omega / \omega=44 \%$ FWHM is comparable to that in sample WP1. From a comparison of the red-shifted WP2 spectrum to theory (see Section 6.4), we deduce a larger rod height in crystal WP2 $\bar{c}=200 \mathrm{~nm}$ (see Fig. 6.3). In both crystals we observe small peaks at frequencies above the stop band, which we attribute to the super structure in the crystal.

The high reflectivity and large relative widths of the stop bands of both crystals show that the crystals interact strongly with the light, in agreement with band gap behavior. The relative width of the stop band of our samples, $\Delta \omega / \omega=44 \%$ is considerably larger than the stop gap width of 30\% that was obtained from band structure calculations on woodpile structures [22]. We note that our crystals do not have a cubic symmetry, but are orthorhombic. Band structure calculations based on the orthorhombic sample of Ref. [124], resulted in an increased calculated width of the stop gap along $\Gamma$-X direction $\Delta \omega / \omega=40 \%$. It is likely that a similar effect takes place in our samples, resulting in a larger width than expected for cubic crystals. A maximum change in the reflectivity is expected near steep slopes in the reflectivity spectrum of the crystal. On such steep edges, even a small shift of the stop band, results in large reflectivity changes. Sample WP1 has a higher reflectivity and sharper band edges of the stop band in the $\{001\}$ direction compared to sample WP2 (see Fig. 6.5). Therefore, we selected sample WP1 for our switching measurements.

The reflectivity experiments on sample WP1 were repeated for both polarizations using the polarized output of our OPAs. For light polarized parallel to the upper rod layer pronounced dips in the high-reflectivity range in the middle of the stop band were observed that are probably due to the superstructure in the crystal [126]. To avoid such features in the switching measurements, the polarization was chosen such that the E-field was aligned perpendicular to the rods in the top layer. The OPA spectra were found to agree with the unpolarized spectra shown in Fig. 6.5, if the average of the two polarizations is taken, and both polarizations contribute equally. 


\subsubsection{Discussion of the nature of the observed stop band}

To understand the nature of the observed stop band in crystal WP1, we try to relate it to a specific set of lattice planes in the crystal by comparing its center position to Bragg's law adapted for photonic crystal (see Ref. [14]). Naively, one would expect that the peak is a direct result of the Bragg reflection from lattice planes normal to the incident beam. The Si filling fraction for sample WP1 is $\Phi_{S i}=a / \bar{b}=$ $27 \%$ resulting in an effective refractive index $n_{a v}=\sqrt{\bar{\epsilon}}=1.96$. By inserting $n_{a v}$, and the $\omega_{\text {center }}=7135 \mathrm{~cm}^{-1}$ into Eq. 1.2, we deduce a lattice spacing of $\mathrm{d}=358 \mathrm{~nm}$. The lattice planes whose spacing is closest to $358 \mathrm{~nm}$, are the $\{002\}$ planes that are spaced by twice the rod thicknesses $2 \times \overline{\mathrm{c}}=310 \mathrm{~nm}$. From diffraction theory [3], however, it is well known that the $\{002\}$ reflection of a monatomic diamond crystal is forbidden, since the corresponding structure factor is zero. We can exclude the role of both the non-spherical "atoms" in our woodpile crystal as well as of the orthorhombic distortion, since neither of them breaks the symmetry that causes the structure factor for the $\{002\}$ planes to be zero. Our measured reflection peak is much broader and also occurs at a different frequency than predicted by Bragg's law. Hence, we suppose that the observed stop band must be a combination of stop bands in other lattice directions. This hypothesis is supported by previous angle resolved transmission measurements on woodpile crystals by Ref. [26], where a red-shift is apparently combined with a splitting of the stop band for angles of incidence moving away from the $\{001\}$ direction towards the $\{110\}$ direction. A stop band that is a result of a reflection from the $\{002\}$ planes only, would demonstrate a blue-shift without splitting, according to Bragg's law. We therefore conclude that Si woodpile structures are truly $3 \mathrm{D}$ photonic crystals.

\subsection{Comparison to exact modal method theory}

This Section briefly describes the exact modal method calculations that were used to calculate reflectivity spectra of our woodpile photonic crystals. Because each individual layer in a woodpile crystal is a grating that is effectively infinite in the lateral directions, such crystals can be described as a stack of gratings. Consequently, we can use the so-called 'Exact Modal Method' (EMM) to solve Maxwell's equations for the woodpile crystal [125]. The programme used to calculate reflectivity spectra is called WPC ('woodpile crystal'), and is described in more detail in Appendix B. The algorithm was developed by Ref. [125], and is designed and optimized to obtain reflectivity spectra of stacked gratings and therefore also of woodpile crystals. Input parameters to the programme are: rod dimensions, the 
lateral alignment of the layers of rods, and the complex refractive index of $\mathrm{Si}$ in the crystal. The number of layers in the crystal is finite, and the thickness of each layer can be specified separately. The EMM method assumes that the crystal is infinite in the lateral dimensions, but finite in the direction normal to the sample interface. We have tested the numerical stability of the WPC model by comparing the obtained reflectivity spectrum to analytical results on a 1D Bragg stack (see Appendix B). We find that our WPC model reproduces the exact TM model results for a simple 1D Bragg stack within $\Delta R=0.2 \%$ for frequencies up to $9000 \mathrm{~cm}^{-1}$ (see Fig. B.1).

There are four important reasons why we have used this programme to model our switched woodpile photonic crystals: first of all, due to the limited number of layers in the crystal, we can couple the equations for the internal and the external fields, and thus obtain a reflectivity spectrum. The calculated spectra allow a direct comparison between theoretical and experimental reflectivity data. Note that such a comparison is much harder for opaline structures, whose structure is much more difficult to model, certainly with a stacked grating model [127]. Secondly, the simple 1D Bragg description breaks down in our 3D crystals [14], since it cannot correctly predict the width nor the center frequency of the stop band in our crystals (see Section 6.3). Thirdly, our model permits complex refractive indices as well as dispersion $n_{S i}^{\prime}(\omega)+i n_{S i}^{\prime \prime}(\omega)$ to be taken into account, which is important in the interpretation of our switched crystals, since the induced refractive index changes are both highly dispersive and complex. Finally, the reflectivity spectra cannot be interpreted by comparison to band structure calculations, since such calculations are based on infinitely large samples, while our sample is finite.

We compare the calculated spectra to the linear reflectivity measurements on sample WP1 and WP2 shown in Fig. 6.5. We use structural information from Fig. 6.3 to model the crystal: the rod width in both crystals was taken to be $a=180 \mathrm{~nm}$, while the rod height in sample WP1 was taken to be $\bar{c}=155 \mathrm{~nm}$. In our calculations, we make three assumptions: firstly, we neglect the superstructure in our crystal; consequently the lateral spacing between the rods is $\bar{b}=b_{1}=b_{2}=650 \mathrm{~nm}$. Secondly, we assume a refractive index $\mathrm{Si} n_{S i}=3.45$ that does not depend on frequency. Finally, we assume that the lateral alignment of the rods in each layer of the crystal is perfect. Since we compare our calculations to unpolarized experimental data, the spectra were obtained by taking the average of two polarizations.

The calculated reflectivity spectrum in the $\{001\}$ direction for crystal WP1 is shown as a dashed curve in Fig. 6.5A. The shape of the calculated stop band compares well to unpolarized FT-IR reflectivity data; the calculation predicts the low frequency edge of the stop band within $100 \mathrm{~cm}^{-1}$, and the high frequency edge 
within $300 \mathrm{~cm}^{-1}$. The calculated peak reflectivity is within $1 \%$ of the measured value. Note that the dispersion of the refractive index of $\mathrm{Si}$ is neglected in our calculation. It is therefore remarkable that our simplified calculation gives a good prediction of the reflectivity stop band of our unswitched crystal.

The same method was used to interpret the reflectivity spectrum for sample WP2, shown as the dashed curve in Fig. 6.5B. The lateral spacing of the rods in sample WP2 is reduced to $\bar{b}=b_{1}=b_{2}=600 \mathrm{~nm}$. We find reasonable agreement between the calculated and the experimental FT-IR spectra by choosing a rod height of $\bar{c}=200 \mathrm{~nm}$ (which was not measured by SEM). The calculation predicts the low frequency edge of the stop band within $100 \mathrm{~cm}^{-1}$, and the high frequency edge within $300 \mathrm{~cm}^{-1}$. The calculated peak reflectivity of sample WP2 is $10 \%$ higher than than the measured data. Despite the simplifications in our model, the overall shape of the calculated stop band agrees reasonably with the experiment.

To get a better agreement between model and experiments, we make four important improvements to the calculations: First of all, the superstructure that was observed in SEM images was included in the description of each layer of rods. Secondly, we also include the slight misalignment of the layers with respect to each other (exemplified by Fig. 6.2) in the model. Thirdly. the resulting calculated reflectivity spectra were convoluted with the measured FWHM of the Topas OPA of $\Delta \omega / \omega=1.44 \%$ to allow direct comparison to the experimental data. Finally, we including the dispersion in the Si backbone of the crystal. $n_{S i}^{\prime}(\omega)+i n_{S i}^{\prime \prime}(\omega)$. The dispersion, and the intrinsic absorption of the unswitched $\mathrm{Si}$ were obtained from Ref. [96]. The resulting calculated reflectivity spectrum is compared to the OPA reflectivity data in Fig. 6.6. The calculation predicts the low frequency edge of the stop band within $100 \mathrm{~cm}^{-1}$, and the high frequency edge within $250 \mathrm{~cm}^{-1}$. The calculated peak reflectivity is within $1 \%$ of the measured data. It is gratifying that the position and width of the stop band in our measurements and the theory agree well, since no parameters were freely adjusted.

Since we also want to calculate switched reflectivity spectra, we have also modeled the nonlinear dispersion in the crystal. It appears that the optical properties of excited Si are highly dispersive, and well described by the Drude model (see Appendix A). The Drude model allows us to obtain a complex refractive index $n_{S i}^{\prime}\left(\omega, N_{e h}\right)+i n_{S i}^{\prime \prime}\left(\omega, N_{e h}\right)$ at a given probe frequency $\omega$, and a carrier density $N_{e h}$. In the Drude description, the induced absorption is related to the $N_{e h}$ through the Drude scattering time $\tau_{D}$ (see Appendix A). A value of $\tau_{D}=10 \mathrm{fs}$ was found to provide a best fit to the data on our polycrystalline Si crystals. We also include the two-photon excitation of free carriers (see Chapter 3). The resulting model for the reflectivity of switched woodpile photonic crystals contains only two experimen- 
tally constrained parameters: $\beta$ and $\tau_{D}$.

\subsection{Experimental results}

\subsubsection{Stop band shift}

The free-carrier induced blue-shift of the stop band is visualized clearly in Figure 6.6, which shows OPA data of the pumped and unpumped reflectivity of a woodpile crystal WP1, square C3 at normal incidence. The probe light was polarized perpendicular to the rods in the top layer of the crystal. Three different scanning ranges (idler, signal, and second harmonic of idler) of the probe OPA were used to allow an octave-broad measurement of the complete stop band. The OPA experiment could not be done in one run, since a realignment of the setup was needed for each scanning range. This realignment results in slight discontinuities in the ranges indicated by the gray ovals, caused by a systematic error $(\Delta R= \pm 5 \%)$ in the reference spectra. The unpumped, polarized reflectivity spectrum shows a broad stop band with a width of $50 \%$ FWHM, which is larger than the width $\Delta \omega / \omega=$ $44 \%$ that was found in unpolarized FT-IR experiments in Section 6.3. We have no conclusive explaination for the mismatch in stop band width. Possibly, the mixing of both polarizations in the FT-IR measurements plays a role. Also, the spectrum in Fig. 6.6 may be slightly broadened by the $1.44 \%$ linewidth of our OPA.

The dashed curve in Fig. 6.6 represents a reflectivity spectrum calculated by the WPC algorithm, that includes all structural data of sample WP1 C3, as well as the dispersion of $\mathrm{Si}$ [96]. The resulting calculated reflectivity spectra were convoluted with the measured FWHM of the Topas OPA of $\Delta \omega / \omega=1.44 \%$ to allow direct comparison to the experimental data. The calculation shown in Fig.6.6 demonstrates the good agreement between calculation and experimental data for the position; the prediction of the position of both edges is accurate within $150 \mathrm{~cm}^{-1}$ and the calculated peak reflectivity is accurate within $1 \%$. A remaining difference is the calculated trough in the reflectivity near $7000 \mathrm{~cm}^{-1}$, which is not observed in any of our measurements. The origin of this trough is discussed in Section 6.6.

In the same run, the crystal was pumped at pump frequency $\omega_{\text {pump }}=5000 \mathrm{~cm}^{-1}$, below the stop band, ensuring a homogeneous distribution of pump light. Pumped reflectivity spectra at 1 ps after excitation are shown as closed circles in Fig. 6.6. The switched stop band is blue-shifted with respect to the unswitched band. Since the whole stop band is so broad, the large switching effect is subtle to discern in this representation. An any rate, the shift causes a large decrease in reflectivity at the red edge of $\Delta R=-10 \%$ and an increase on the blue edge of $\Delta R=+5 \%$, shown 


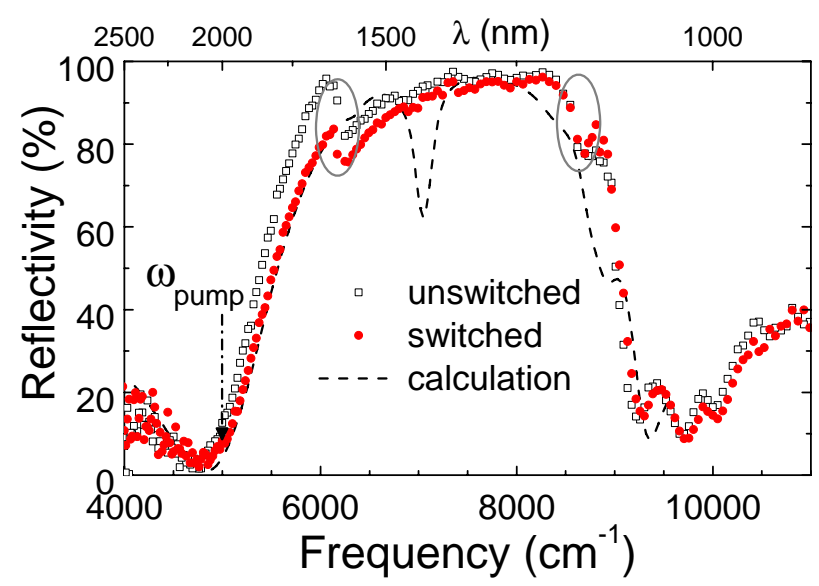

Figure 6.6: Unswitched (open squares) and switched (closed circles) reflectivity spectra of crystal WP1, square C3 at normal incidence. The gray ovals indicate the edges of the scanning ranges of the OPA. The dashed curve represents the reflectivity as calculated by the WPC algorithm, taking all structural imperfections of the crystal into account and convoluted with the OPA line width, showing good agreement with the unswitched spectrum. The pump irradiance for the switched spectrum was $I_{0}=17 \pm 1 \mathrm{GWcm}^{-2}$ on the red side, $I_{0}=16 \pm 1 \mathrm{GWcm}^{-2}$ on the central part, and $I_{0}=16 \pm 1 \mathrm{GWcm}^{-2}$ on the blue side of the stop band. The arrow indicates the pump frequency $\omega_{\text {pump }}=5000 \mathrm{~cm}^{-1}$. The switched spectra were measured after the initial Kerr effect, at a pump-probe time delay of 1 ps on the red part, $300 \mathrm{fs}$ on the central part and $1.5 \mathrm{ps}$ on the blue part of the spectrum.

in Fig. 6.7B. We observe two important effects. Firstly, the measured shift of the red edge of the gap is 5.6 times larger than the shift of the blue edge. At the low frequency edge, $\omega=5500 \mathrm{~cm}^{-1}$, the shift is found to be $130 \pm 5 \mathrm{~cm}^{-1}$ or $2.4 \%$, while at the high frequency edge, $\omega=9000 \mathrm{~cm}^{-1}$ it is $\Delta \omega=38 \pm 8 \mathrm{~cm}^{-1}$ or $0.42 \%$. Section 6.6.4 will elaborate on the origin of this large difference. Secondly, the peak reflectivity of the switched structure remains high: at central frequencies near 7000 $\mathrm{cm}^{-1}$, the peak reflectivity of the stop band decreases by less than $\Delta \mathrm{R} / \mathrm{R}=-1 \%$. Our observations confirm that large changes in the stop band position can be achieved while the induced absorption remains small.

\subsubsection{Differential reflectivity versus probe delay and wavelength}

The induced stop band shift is large, but small compared to the band width of the entire stop band. Therefore we also plot the reflectivity changes in the range of the stop band. 

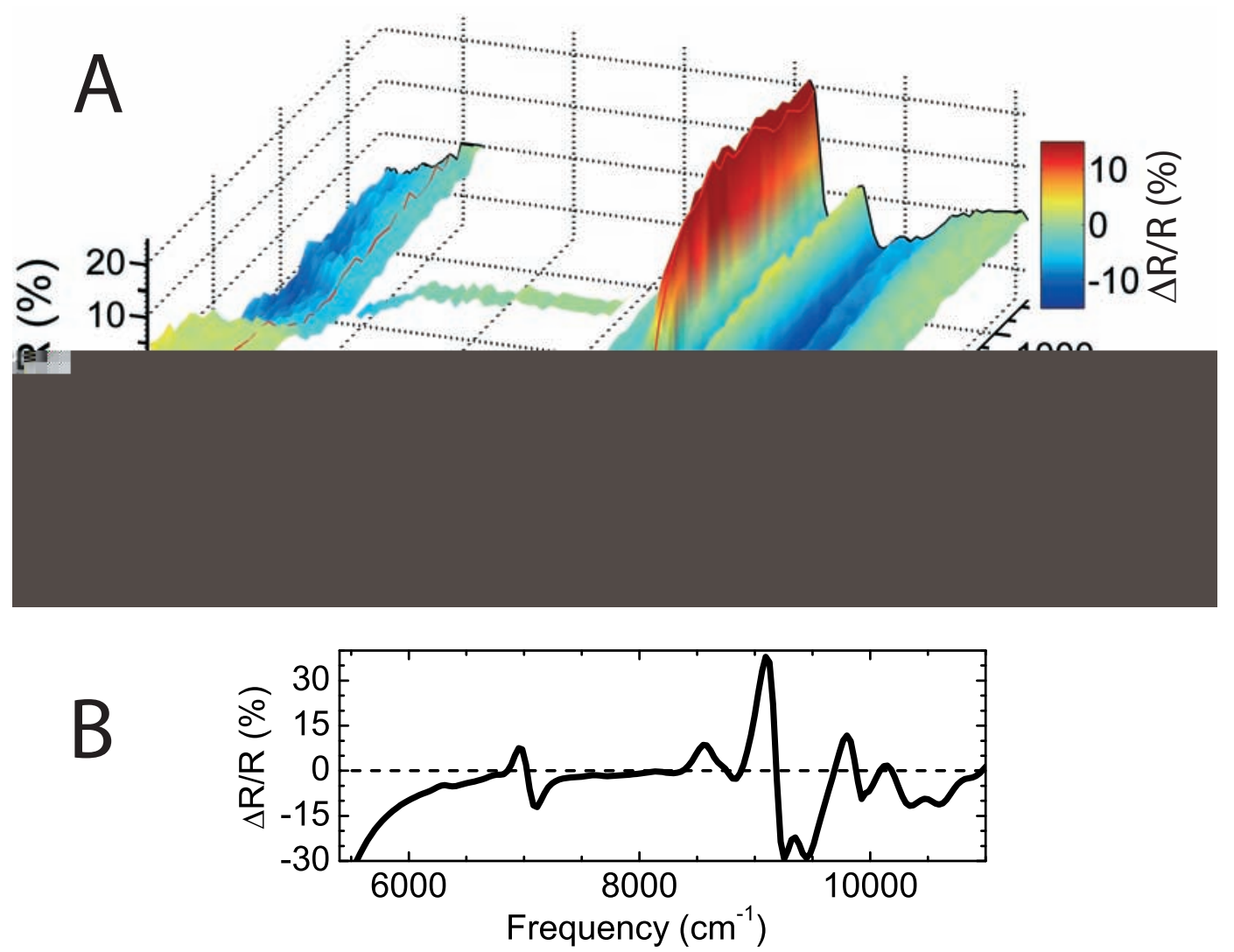

Figure 6.7: (A) (color online) Differential reflectivity versus both probe frequency and probe delay. The pump peak irradiance was $I_{0}=17 \pm 1 \mathrm{GWcm}^{-2}$ on the red part, $16 \pm 1 \mathrm{GWcm}^{-2}$ on the central part, and $16 \pm 1 \mathrm{GWcm}^{-2}$ on the blue part of the spectrum. The probe delay was varied in steps of $\Delta t=$ $50 \mathrm{fs}$. The probe wavelength was tuned in $\Delta \lambda=10 \mathrm{~nm}$ steps in the low, and central range, and in 5 $n m$ steps in the high-frequency range. In the central part of the stop band, $\Delta R / R(\omega)$ was measured at both negative delays and a positive delay of $300 \mathrm{fs}$. The red curves indicate fixed frequency curves along which extensive delay traces were measured. (B) Differential reflectivity changes versus probe frequency, calculated from exact modal method theory that includes the Drude model, and obtained by ratioing to the unswitched calculated spectrum shown in Fig. 6.6. The relative changes at the stop band edges agree quantitatively with the measured data in $(A)$.

Fig. 6.7A shows the differential reflectivity $\Delta R / R(\tau, \omega)$ of sample WP1 square $C 3$ as a function of probe delay $\tau$ over an octave-broad probe frequency range $\omega$. A data set of 1000 pulse events was used for each $\Delta R / R(\tau, \omega)$ point on the plot surface shown in Fig. 6.7A. The differential reflectivity was obtained by ratioing the switched and 
unswitched data that were measured in a single run for each $\Delta R / R(\tau, \omega)$ point. The differential spectra were reproduced on other positions on the crystal surface of square C3. We observe strong decreases as well as increases in the reflectivity, especially at the stop band edges that are indicated by the red curves in Fig. 6.7.

At 1 ps after excitation, we observe large and highly dispersive reflectivity changes. The reflectivity at the low frequency edge of the stop band $\left(6000 \mathrm{~cm}^{-1}\right)$ displays a large and ultrafast decrease $\Delta \mathrm{R} / \mathrm{R}=-8 \%$. This decrease is indicative of a shift of the stop band edge to higher frequencies. We attribute this effect to excited free carriers that reduce the refractive index of the $\mathrm{Si}$. At intermediate frequencies near $7000 \mathrm{~cm}^{-1}$, at the flat central part of the stop band, we observe a small decrease in the peak reflectivity by less than $\Delta R / R=-1 \%$. We ascribe this effect to a small amount of induced absorption in the crystal. At probe frequencies above the gap, near $9400 \mathrm{~cm}^{-1}$, strong variations in $\Delta \mathrm{R} / \mathrm{R}$ with frequency occur that are related to the shift of the superstructure feature (see Fig. 6.5(A)). At the blue edge of the gap at $9170 \mathrm{~cm}^{-1}$, we observe a large increase in reflectivity up to $\Delta \mathrm{R} / \mathrm{R}=25 \%$, which indicates that the blue edge of the gap also shifts to higher frequencies. We conclude that the entire stop band is shifted towards higher frequencies at 1 ps after excitation.

Unexpectedly, we observe a sharp decrease in reflectivity on both edges of the stop band near zero delay at $\tau \approx 200 \mathrm{fs}$. Since the width of the trough is comparable to the pump pulse width, we attribute the effect to an electronic Kerr nonlinearity. Since the Kerr effect only occurs at temporal overlap of pump and probe, the sharp minimum could also be used to correct our temporal calibration for dispersion in the probe path. Near both stop band edges, we observe that at short time delays, the measured decrease $\Delta \mathrm{R} / \mathrm{R}$ at the peak is smaller than the corresponding decreases at the edge. We therefore conclude that, the edges move towards each other, resulting in a narrowing of the gap. The observation can not be explained by induced absorption, since absorption would lead to an equal reflectivity decrease $\Delta R / R$ both at the edge of the stop band as well as on top of the peak (see Fig. 6.15). Fig. 6.8 summarizes our observations: the unswitched stop band (top panel) is narrowed at time delays below $200 \mathrm{fs}$ (middle panel) subsequently, the entire stop band is shifted towards higher frequencies (lower panel). These non-monotonic effects were not expected by Ref. [54], and lead to the striking conclusion that a single pump pulse suffices to switch the LDOS inside our crystals up and down. 


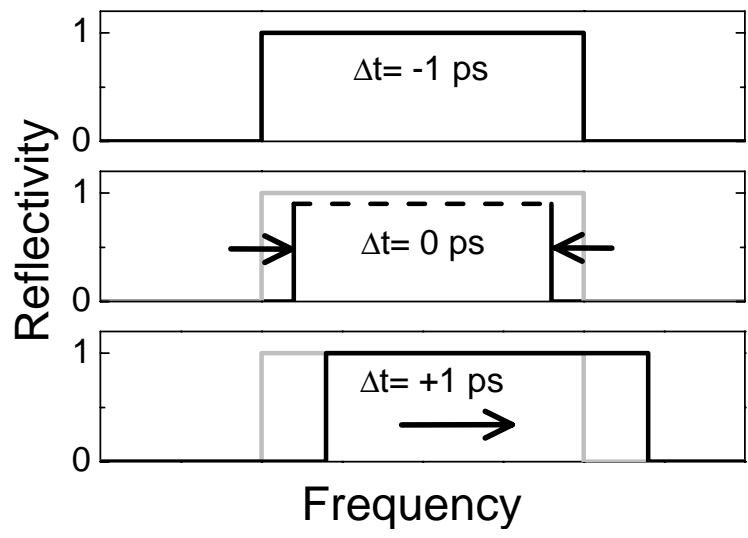

Figure 6.8: Cartoon depicting the temporal behavior of the stop band with increasing probe delay as deduced form the measurements shown in Fig. 6.7. The upper panel shows the unswitched stop band for negative delays. For $\Delta t \approx 0$, the stop band narrows (middle panel) while for larger $\Delta t$ the stop band shows a blue-shift (lower panel).

\subsubsection{Time-resolved measurements}

To study the intricate ultrafast behavior in more detail, we have measured the transient absolute reflectivity changes $\Delta \mathrm{R}$ at two characteristic frequencies. Fig. 6.9 shows delay traces over an extended time range that were measured at probe frequencies at the red and blue edge of the stop band, indicated by the red traces in Fig. 6.7B. At the blue edge, a rapid decrease to $\Delta \mathrm{R}=-1.7 \%$ appears within 190 $\mathrm{fs}$, followed at $270 \mathrm{fs}$ by an increase to $\Delta \mathrm{R}=5 \%$ within $500 \mathrm{fs}$. The decrease is due to an electronic Kerr effect and the increase is attributed to optically generated free carriers. The free-carrier effect subsequently decays exponentially with a decay time of $18 \pm 1$ ps. The reflectivity at the red edge decreases by $\Delta R=-12 \%$ within 1 ps due to a Kerr nonlinearity, followed by a free-carrier effect. At 2.5 ps after the excitation, the effect on the red edge decays exponentially to $\Delta R=-1 \%$ with a decay time of $16 \pm 2$ ps. The decay times of about 18 ps are much shorter than carrier relaxation times in bulk $\mathrm{Si}$, likely since our photonic crystals are made of polycrystalline silicon, whose lattice defects and grain boundaries act as efficient carrier recombination traps [110]. On the other hand, the decay time is longer than that of amorphous $\mathrm{Si}$, in which recombination times as short as a few picoseconds have been observed [128]. The relatively fast decay time implies that switching could potentially be repeated at a rate above $25 \mathrm{GHz}$, which is relevant to possible future switching and modulation applications and will be discussed in Section 6.6. 

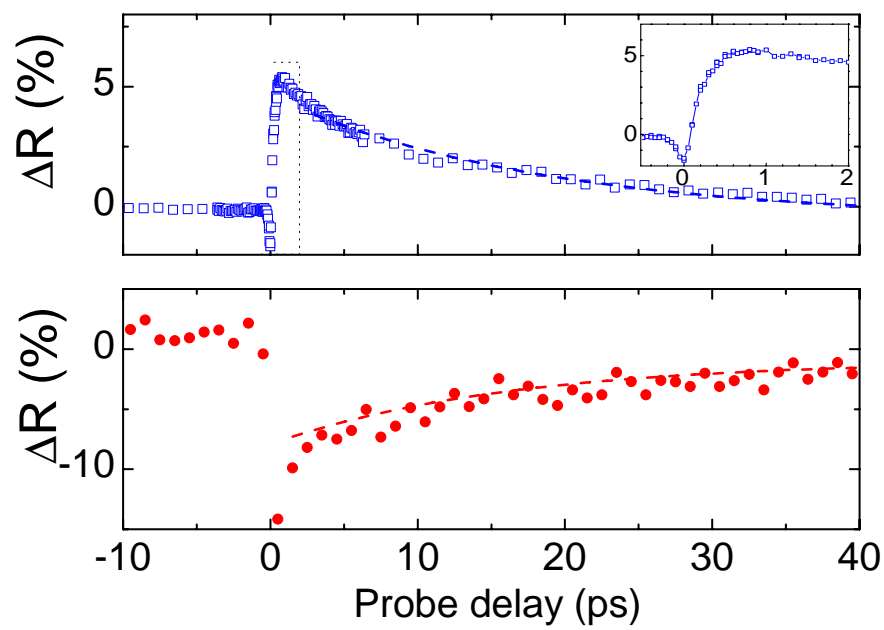

Figure 6.9: (color online) Absolute reflectivity changes versus probe delay at frequency $\omega_{\text {blue }}=9174$ $\mathrm{cm}^{-1}$ at the blue edge of the gap (upper panel) and $\omega_{\text {red }}=5882 \mathrm{~cm}^{-1}$ at the red edge (lower panel) of the gap. The pump irradiance was $I_{0}=16 \pm 1 \mathrm{GWcm}^{-2}$. The dashed curves are exponential fits with a decay time of 18 ps (upper panel) and 16 ps (lower panel).

\subsubsection{Free-carrier effects}

To verify that the effects after 1 ps are induced by free carriers excited by twophoton absorption, we plot the frequency shift of the blue edge of the stop band versus peak pump power squared $I_{0}^{2}$ in Fig. 6.10. In the same graph we have also plotted the shift of the reflectivity feature at $9750 \mathrm{~cm}^{-1}$. Both features shift linearly with the peak pump power squared, which confirms that a two-photon process (i.e. a $\chi^{(5)}$ nonlinear process) is indeed the dominant excitation mechanism. Since the reflectivity changes remain after the pump pulse and decay with times in between bulk and amorphous silicon carrier recombination, it is safe to conclude that indeed free carriers are excited.

To allow a Drude description of our samples, we wish to estimate the carrier density $N_{e h}$. Therefore, we first deduce the magnitude of refractive index change $\Delta n_{S i}^{\prime} / n_{S i}^{\prime}$ from the observed stop band shift in our crystals. We use the WPC model to calculate reflectivity spectra at various refractive indices $n_{S i}^{\prime}$, and investigate the relation between the stop band shift $\Delta \omega / \omega$ and the refractive index change $\Delta n_{S i}^{\prime} / n_{S i}^{\prime}$. Fig. 6.11A shows the resulting reflectivity spectra for a perfectly aligned woodpile crystal at two typical refractive indices $n_{S i}=3.45$ (solid curve) and $n_{S i}=3.35$ (dashed curve). We observe that the stop band shifts towards higher frequency as $n_{S i}$ is 


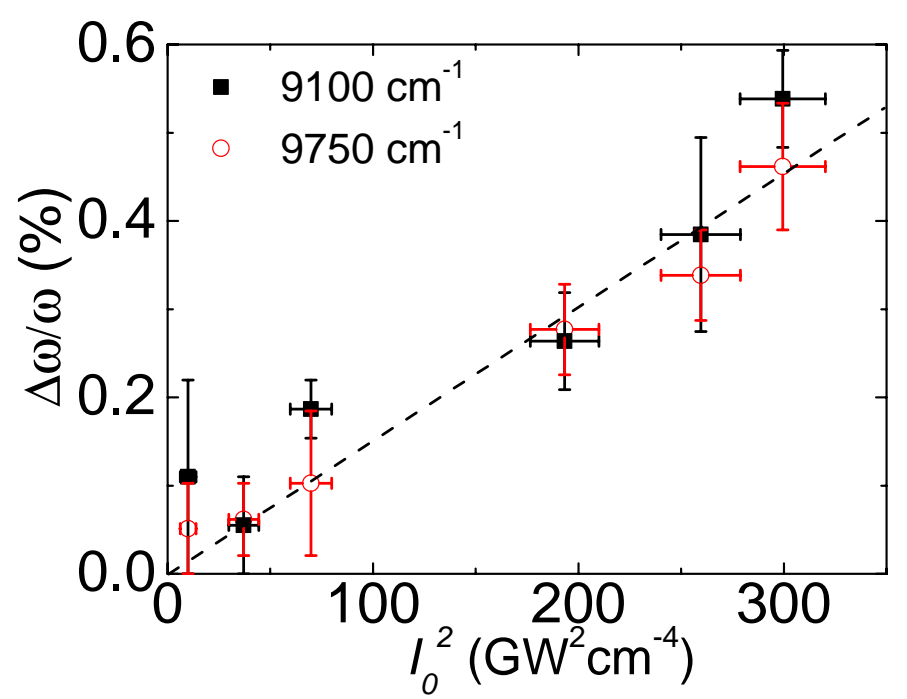

Figure 6.10: (color online) Squares: measured shift $\Delta \omega / \omega$, at $\omega=9100 \mathrm{~cm}^{-1}$ on the blue edge of the stop band at 1 ps after excitation versus $I_{0}{ }^{2}$. Diamonds: $\Delta \omega / \omega$ measured at $\omega=9750 \mathrm{~cm}^{-1}$. The maximum observed shift is $\Delta \omega / \omega=0.54 \%$. The dashed curve serves to guide the eye.

reduced, as expected from Bragg's law. In this calculation, which does not take into account dispersion both the low and the high frequency edges of the stop band shift by the same amount, which is inconsistent with the highly dispersive switching effects that we observed in Fig. 6.6.

From each calculated spectrum, the center position of the stop band was obtained, and its blue-shift was calculated by comparison to the unswitched spectrum. Fig. $6.11 \mathrm{~B}$ shows the shift in center position $\Delta \omega_{\text {center }} / \omega_{\text {center }}$ for both polarizations, plotted versus $\Delta n_{a v} / n_{a v}$. Remarkably, the results coincide with the line $\Delta \omega_{\text {center }} / \omega_{\text {center }}=\Delta n_{a v} / n_{a v}$ (dashed line). Thus, in absence of absorption, the induced shift in our woodpile crystals is in good agreement with Bragg's law up to large refractive index changes $\Delta n_{a v} / n_{a v}=5 \%$. To allow a comparison of our experiments to theory, we have also calculated how the stop band shifts with refractive index change if bulk dispersion and the exact structure of our crystals are taken into account. We deduce that the refractive index change in the Si backbone is roughly equal to the induced relative shift of the peak $\Delta \omega / \omega=0.95 \times \Delta n_{S i} / n_{S i}$.

From measured switched reflectivity spectra at 1 ps (see Fig. 6.6), we have deduced a large maximum shift of $\Delta \omega_{\text {red }} / \omega_{\text {red }}=2.4 \%$ of the red edge, while the blue edge shifts by a much smaller amount: $\Delta \omega_{\text {blue }} / \omega_{\text {blue }}=0.54 \%$. The highly dispersive 


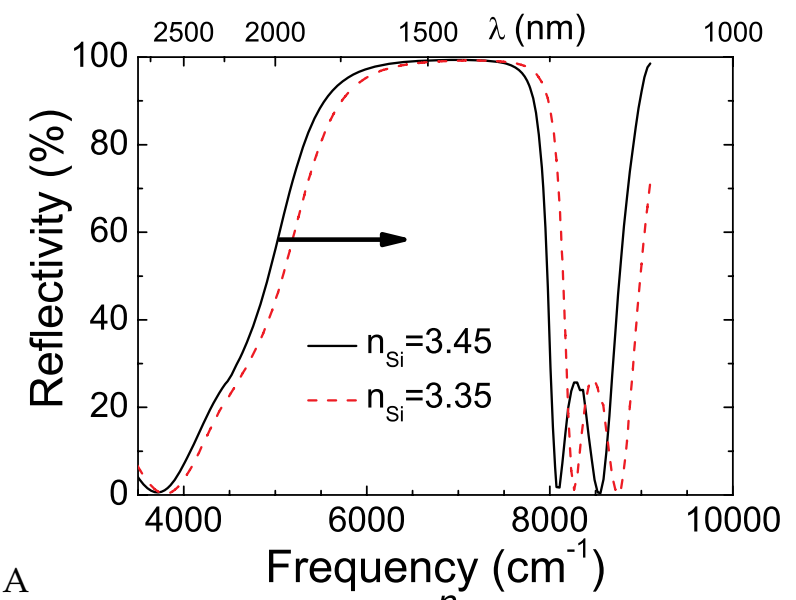

A

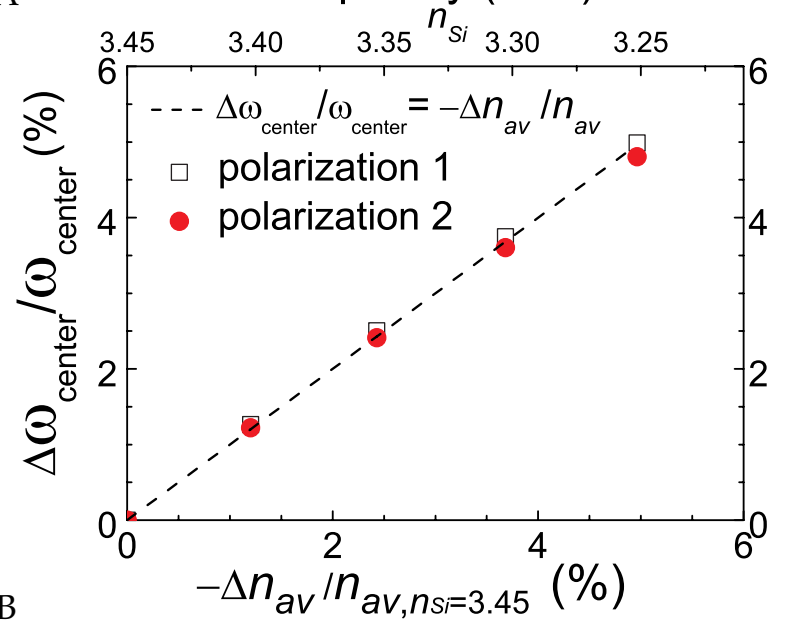

Figure 6.11: (A) Calculated reflectivity spectra of a perfect five-layer thick Si woodpile crystal with dimensions as specified in the text. The incident light is polarized parallel to the rods in the upper layer. The refractive index is changed from $n_{S i}=3.45$ (solid curve) to $n_{S i}=3.35$ (dashed curve). The stop band clearly shifts to higher frequency with decreasing refractive index change $\Delta n_{S i}$, as expected from Bragg's law. (B) Shift of the stop band center versus change in average refractive index for both polarizations. The refractive index $n_{S i}$ was varied from 3.45 to 3.25. The relative shift $\Delta \omega / \omega$ is proportional to the change $-\Delta n_{a v} / n_{a v}$ in agreement with Bragg's law.

refractive index change is consistent with a dispersive Drude description of free carriers (see Appendix A).

A Drude description was used to estimate the carrier density $N_{e h}$. The shift at the blue edge of the gap at $\omega=9425 \mathrm{~cm}^{-1}$ amounts to $\Delta \omega / \omega=0.54 \%$ (see Fig. 6.10). Using the proportionality factor discussed above, we deduce a change in refractive index 
$\Delta n_{S i} / n_{S i}=0.54 / 0.95=0.57 \%$. Next, we use a Drude model with parameters $m_{\text {opt }}^{*}=$ 0.15 [103], and $\tau_{D}=10 \mathrm{fs}$ [91] to calculate the dispersion for excited polycrystalline $\mathrm{Si}$ at two different carrier densities (see Fig. 6.12) to estimate the carrier density in our experiment. In Fig. 6.12, the horizontal dotted line at $\Delta n_{S i} / n_{S i}=0.57 \%$ line and the vertical $\omega=9425 \mathrm{~cm}^{-1}$, intersect just below the Si dispersion curve for $N_{e h}=$ $2 \times 10^{19} \mathrm{~cm}^{-3}$. Therefore we estimate that the carrier density in the excited sample is: $N_{e h}=2.1 \times 10^{19} \mathrm{~cm}^{-3}$. At this carrier density, the Drude model expects a refractive index change $\Delta n_{S i} / n_{S i}=1.8 \%$ at the red edge of the stop band, which is smaller than the change $\Delta n_{S i} / n_{S i}=2.5 \%$ that we have deduced from the measured shift of the red edge of the stop band in Fig. 6.6. The carrier density in the experiment at the low frequency edge may therefore have been even higher. We conclude that a Drude description with a carrier density $N_{e h}=2.1 \times 10^{-19} \mathrm{~cm}^{-3}$ provides quite a reasonable description of the dispersion in our switched crystals.

The obtained carrier density $N_{e h}=2.1 \times 10^{-19} \mathrm{~cm}^{-3}$ was used to interpret the observed changes in Fig. 6.7A. Fig. 6.7B shows calculated differential reflectivity $\Delta R / R(\omega)$ obtained by ratioing a calculated reflectivity spectrum that includes Drude dispersion, to that of an unswitched crystal. At the low frequency edge of the stop band the reflectivity decreases by up to $\Delta R / R=30 \%$, while at the high frequency edge, a strong reflectivity increase of $\Delta R / R=37 \%$ is observed. The measured changes in Fig. 6.7 occur at the same frequency positions, but are $\approx 1.5$ times smaller. The feature near $7000 \mathrm{~cm}^{-1}$ in the calculated spectrum is caused by a shift of the trough at that frequency which was not observed in our measurements. In conclusion, by including the free-carrier dispersion in our WPC model via a Drude description, we find quantitative agreement with the measured highly dispersive reflectivity changes on both edges of the stop band.

From the deduced carrier density $N_{\text {eh }}$ and the pump irradiance $I_{0}=17 \pm 1 \mathrm{GWcm}^{-2}$ at $\omega_{\text {pump }}=5000 \mathrm{~cm}^{-1}$ used in the experiment, we derive the two-photon absorption coefficient to be $\beta=60 \pm 15 \mathrm{cmGW}^{-1}$. The corresponding large pump absorption length in the $\mathrm{Si}$ backbone at is $\ell_{a b s}=\left(I_{0} \beta\right)^{-1}=9.3 \mu \mathrm{m}$. The penetration depth of pump light into the crystal is obtained by dividing by the Si filling fraction: $\ell_{\text {pump }}=$ $\ell_{a b s} / \Phi_{S i}=9.3 / 0.27=36.3 \mu \mathrm{m}$, or $>230$ layers of rods, confirming that two-photon excitation of carriers yields much more homogeneously switched crystals than in one-photon experiments $[88,90]$.

\subsubsection{Electronic Kerr effect}

To verify that the reflectivity changes at zero probe delay are indeed related to a $\chi^{(3)}$ Kerr response, delay traces like those in Fig. 6.9 were measured on a separate 


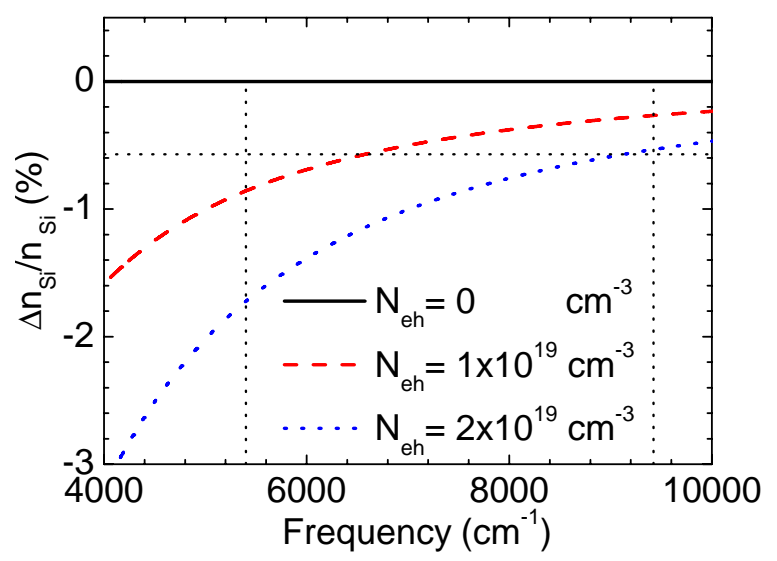

Figure 6.12: Relative refractive index change in Si versus probe frequency at three different carrier densities. The vertical dotted lines indicate the stop band edges $\omega=5400 \mathrm{~cm}^{-1}$ and $\omega=9425 \mathrm{~cm}^{-1}$. The horizontal dotted line indicates the value $\Delta n_{S i} / n_{S i}=0.57 \%$ that was obtained from Fig. 6.10 .

crystal (WP1, square D4). The magnitude of the Kerr change $\Delta R / R$ at $\tau=0$ at the blue edge of the stop band was studied as a function of pump irradiance in Fig. 6.13. 
$6452 \mathrm{~cm}^{-1}$ to be $0.3-0.5 \pm 0.3 \times 10^{-13} \mathrm{~cm}^{2} \mathrm{~W}^{-1}$ by z-scan measurements. Note that the data of Ref. [97] were obtained for $\omega_{\text {pump }}=\omega_{\text {probe, }}$ while in our two-color pump probe experiment, $\omega_{\text {pump }} \neq \omega_{\text {probe }}$. Furthermore, Ref. [97] explicitly studies bound electrons, while our results likely include excite electron effects. Since the Kerr nonlinearity in our samples is larger than expected, it could potentially be used as a separate switching mechanism in our future density of states switching experiment.

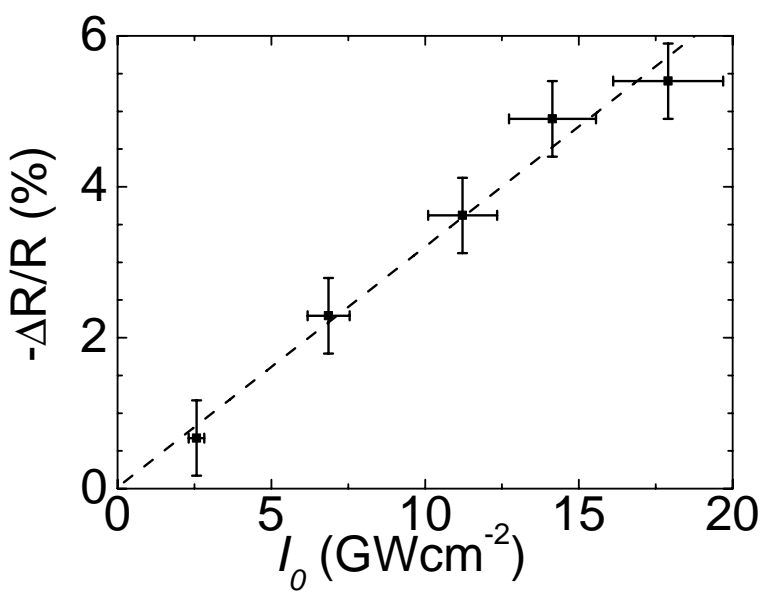

Figure 6.13: (B) Differential reflectivity decrease at $\tau=0$ on the blue edge of the stop band at $\omega=$ $9709 \mathrm{~cm}^{-1}$ versus $I_{0}$. The reflectivity change scales linearly with $I_{0}$, consistent with a third-order Kerr nonlinearity. The dashed line is a least squares fit to the data.

\subsection{Discussion}

In this Section we discuss several aspects of the optical switching of Si woodpile photonic crystals in more detail. Firstly, the observed differences between calculated and measured linear reflectivity spectra are considered. Secondly, we estimate the amount of induced probe absorption in our switched crystals. Thirdly, we discuss the 'switching requirements' mentioned in Chapter 2, and compare them to our experiments. Finally, we briefly discuss possible applications. 


\subsubsection{Comparison of measured and calculated linear reflectivity}

In Fig. 6.6, both a measured and a calculated reflectivity spectrum of sample WP1 were shown. There is reasonable agreement for the position, width, and peak reflectivity of the stop band. Nevertheless, there is also an important difference: our calculation predicts a trough in the reflectivity at $7050 \mathrm{~cm}^{-1}$, while this feature is not observed in the experimental data.

A trough in the stop band was also observed in the spectrum in Fig. 6.14, where the reflectivity for light polarized along the upper layer of rods is shown. The stop band for this polarization is broad (59\% FWHM). A deep and broad trough appears in the stop band at $\omega=7800 \mathrm{~cm}^{-1}$. From the width, we deduce the quality-factor of this resonance to be $Q \sim 14$. The trough at this polarization is related to a superstructure resonance in the crystal, see Ref. [124]. Nevertheless, the polarization related trough does not explain the resonance in our calculation since it occurs at a different frequency. We conclude that interpretation of superstructure related troughs is more subtle than expected.

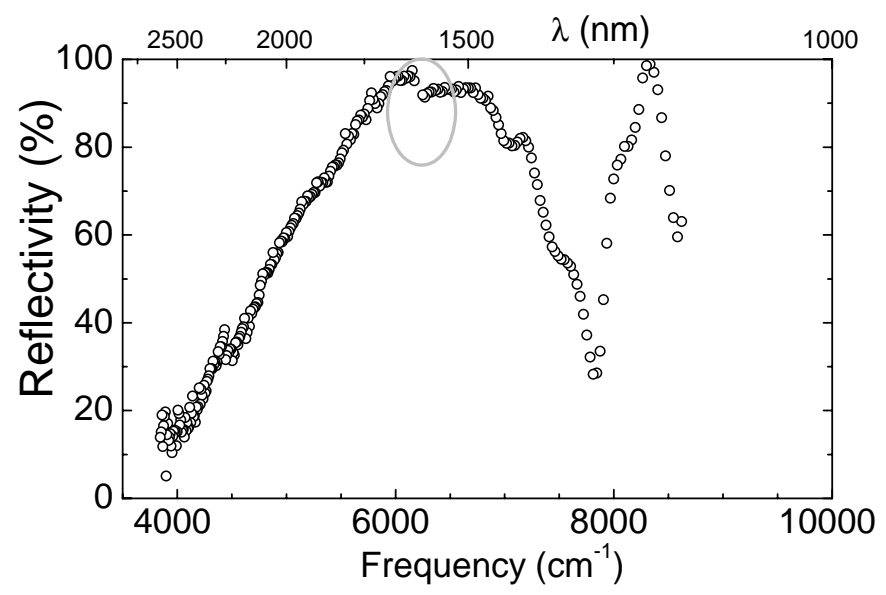

Figure 6.14: Reflectivity spectra for sample WP1, square C3 measured by our OPA. The polarization of the incident field was aligned parallel to the rods in the upper layer of rods in the crystal. The gray oval indicates the edge of the scanning range of the OPA. A 59\% broad stop band occurs between frequencies $\omega=4760 \mathrm{~cm}^{-1}$ and $\omega=8700 \mathrm{~cm}^{-1}$. We observe a deep $(\Delta R \sim 70 \%)$ and $564 \mathrm{~cm}^{-1}$ broad resonance that is centered around $\omega=7800 \mathrm{~cm}^{-1}$. 


\subsubsection{Induced absorption in the switched crystal}

To quantify the induced absorption in our experiments, we compare our data to exact modal method calculations in which the $n_{S i}^{\prime \prime}$ is varied from 0 to 0.1 . Fig. 6.15 shows exact modal method calculations for a structurally perfect woodpile crystal. We have assumed a five-layer woodpile crystals with rod width $\mathrm{a}=180 \mathrm{~nm}$, rod height $\overline{\mathrm{c}}=200 \mathrm{~nm}$, period $\overline{\mathrm{b}}=650 \mathrm{~nm}$ and a complex refractive index $n_{S i}=3.45$ $+\mathrm{i} n_{S i}^{\prime \prime}$. The peak reflectivity of the stop band decreases from $97 \%$ (no absorption) to $73 \%\left(n_{S i}^{\prime \prime}=0.1\right)$. In our experiments (see Fig. 6.6), the maximum decrease in peak reflectivity of the central flat part of the stop band after switching is $\Delta \mathrm{R}=$ $4.6 \%$ near $\omega=6450 \mathrm{~cm}^{-1}$, while at the high frequency side near $\omega=8300 \mathrm{~cm}^{-1}$ the decrease is much smaller $\Delta \mathrm{R}=1.6 \%$. We compare the calculated maximum reflectivity decrease in Fig. 6.15 to the measured decrease $\Delta R=4.6 \%$, and conclude that the induced absorption in the experiment in Fig. 6.6 remains below $n_{S i}^{\prime \prime}=0.015$. Inserting $n_{S i}^{\prime \prime}=0.015$ into Eq.A.9 yields a carrier absorption length $\ell_{c a}>27 \mu \mathrm{m}$ (at $7300 \mathrm{~cm}^{-1}$ ), which equals $>40$ unit cells and thus much more than our sample thickness. Based on this result, we conclude that induced absorption is very small in our switching experiments.

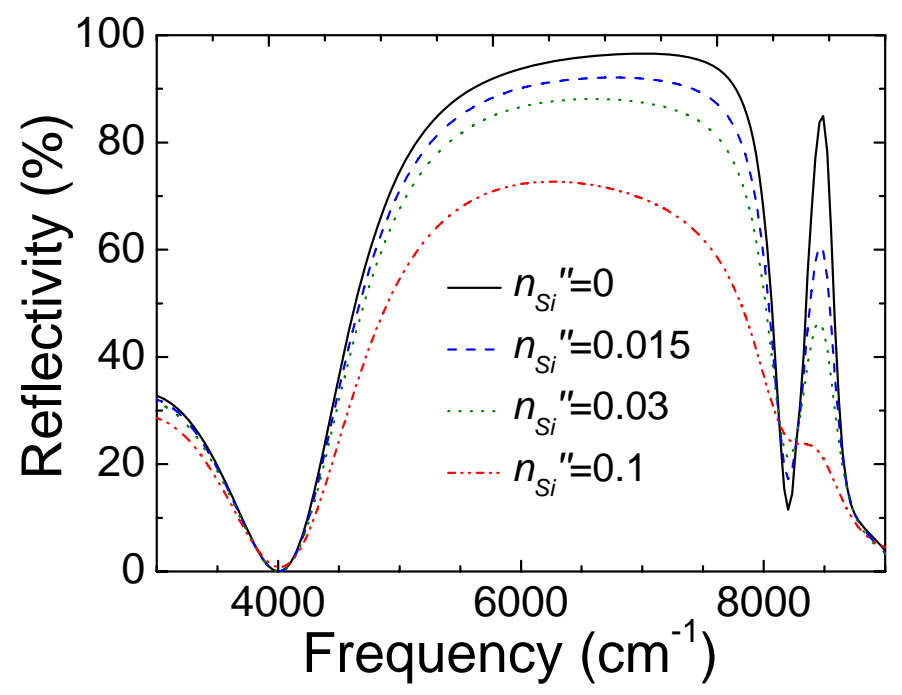

Figure 6.15: Calculated stop bands for a perfect five-layer woodpile crystal with increasing absorption. The real part $n_{S i}^{\prime}$ of the refractive index is 3.45 for all calculations. With increasing absorption, the peak reflectivity of the stop band decreases, and the features at frequencies above the stop band become less pronounced. 


\subsubsection{The 'switching requirements'}

In Chapter 2, quantitative minimum experimental requirements were stated for optical density of states switching experiments. Here, we briefly review the requirements for the experiments presented in this Chapter.

First of all, a large enough refractive index change is needed to induce a measurable change in the LDOS at a fixed frequency. In our experiments, we have observed frequency shifts of $\Delta \omega / \omega=0.54 \%$ at the blue edge of the gap, up to $\omega / \omega=2.4 \%$ at the red edge of the gap (see Fig. 6.6). We will test if the lower limit of the change $(\Delta \omega=0.54 \%)$ is sufficient to induce large changed in the LDOS at a fixed frequency. For small frequency shifts $<2 \%$, the effect of changing the refractive index, can be approximated by a rescaling of the frequency axis in a DOS spectrum [54]. Calculations in Ref. [24] of the DOS inside a perfect and infinitely large woodpile photonic crystal predict large variations of the DOS with frequency at the edges of the band gap. From their data we estimate that a frequency scaling by $\Delta \omega / \omega=$ $0.54 \%$ would cause an increase in the DOS from 0 to $80 \%$ of the vacuum DOS at frequencies at the low frequency end of the band gap of the unswitched crystal. At the high frequency edge of the band gap, the DOS is expected to decrease by a similar amount upon switching. While it is important to note that our crystal is finite in one direction, and therefore strictly speaking does not possess a true band gap, a strong reduction of the local density of states (LDOS) has been predicted in woodpile crystals of several unit cells thick [129]. In absence of a band gap, strong variations of the LDOS are also expected to appear near cavity resonances. We conclude that the refractive index change in our experiments is sufficient to induce large and measurable changes of the LDOS inside woodpile photonic band gap crystals.

The switching time scale of the Kerr effect in our experiment is governed by the pulse duration of the pump pulses: $120 \mathrm{fs}$. The subsequent free-carrier effect, also takes place within a few hundred femtoseconds. Both processes take place at timescales well below the switching time of 1 ps required in Chapter 2. The recombination time of the carriers on the other hand, takes place at a $\sim 20$ ps timescale.

The pump homogeneity length in our switched crystals was deduced from the irradiance scaling of the stop band shift in Section 6.5.4. A 1/e absorption length $\ell_{\text {pump }}=\ell_{\text {abs }}=36.3 \mu \mathrm{m}$ was derived. This absorption length corresponds to $>230$ layers of rods, which is $>57$ unit cells. The homogeneity length of the switched crystal is therefore $\ell_{\text {hom }}>5.7$ unit cells, fulfilling the homogeneity requirement $\left(\ell_{\text {hom }}>5\right.$ unit cells. $)$ for homogeneously switched crystals. 
The probe absorption length $\ell_{c a}$ was found to be more than $27 \mu \mathrm{m}$, or 44 unit cells, which much larger than the required 10 unit cells (see Section 6.6.2). Our samples remain transparent after switching. In conclusion, the experiments presented in this Chapter meet all requirements that were stated in Chapter 2 for density of states switching.

\subsubsection{Asymmetry in the stop band shift}

Here we discuss the asymmetry in the shift on low and high frequency edges of the stop band shown in Fig. 6.6. Relative shifts at the stop band edges $\omega=5500 \mathrm{~cm}^{-1}$ and $\omega=9000 \mathrm{~cm}^{-1}$ were determined from Fig. 6.6 to be $\Delta \omega / \omega=2.36 \%$ and $\Delta \omega / \omega=$ $0.42 \%$ respectively, yielding a large shift ratio of $5.6\left({ }_{-1}^{+1.5}\right)$ between the relative shifts. We discuss possible causes of this effect.

Figure 6.12 demonstrates how the refractive index change depends on probe frequency through the Drude model. The observed change of $\Delta n_{S i} / n_{S i}=-0.57 \%$, at the blue edge $\omega=9425 \mathrm{~cm}^{-1}$ of the stop band corresponds to a larger change of $-1.7 \%$ at the red edge of the stop band $\omega=5500 \mathrm{~cm}^{-1}$. The shift of the red stop band edge is thus expected to be 3.0 times as large as the shift of the blue edge. In the limit of small changes, the induced shift $\Delta \omega / \omega$ of the stop band edges scales linearly with $\Delta n_{S i}^{\prime}$ (see Fig. 6.11B). The observed asymmetry in stop band shift can therefore be partly attributed to the effect of Drude dispersion.

An additional effect that artificially influences the shift ratio is induced absorption in the crystal. On the red edge, induced absorption will appear as an increase the measured blue-shift of the stop band edge, while on the blue edge, induced absorption will decrease the amount of measured shift. Since absorption has an opposite effect on the shift of red-and blue edge, it will result in an elevated shift ratio, and the ratio in real crystals, will thus be larger than predicted from models that do not include induced absorption. The combined effect of Drude dispersion together with the induced absorption are likely the cause of the large difference in frequency shift on both stop band edges.

\subsection{Conclusions and outlook}

In this Chapter, we have demonstrated ultrafast switching and recovery of $\mathrm{Si}$ woodpile photonic band gap crystals at telecom wavelengths by all-optical electronic Kerr- and free-carrier effects. Broadband differential reflectivity spectra in the range of the band gap were measured as a function of probe delay time. The observed large reflectivity changes are studied as a function of pump irradiance. 
From our analysis we clearly separate optically excited free-carrier and a Kerr nonlinearity. The free-carrier induced frequency shift of the band gap as a function of pump irradiance agrees well with exact modal method calculations which include the Drude model.

In our switching experiments, we find unexpected non-monotonic physics: at short fs times, the photonic gap narrows, followed at longer ps times by a blue-shift of the gap. In other words, the blue edge of the gap shifts to lower frequency within the pulse duration, and subsequently shifts to higher frequency compared to the unswitched state, while the red edge only shifts to higher frequency. Consequently, the density of states near the blue edge of the band gap behaves more complex than predicted [54]. Consequently, we propose that a single pulse suffices to switch the LDOS on by the Kerr effect, and rigorously off by free carriers on times governed by the pulse duration. Clearly, such versatile temporal density of states control will open exciting opportunities for timed QED light sources whose emission is switched in absence of a cavity. The experiments presented in here are an important step towards further understanding and dynamic control over the optical density of states in photonic crystals. 


\section{Chapter}

\section{Ultrafast switching of Si inverse opals}

We present ultrafast optical switching experiments on Si inverse opals. Switching is achieved by exciting free carriers by a two-photon process. We demonstrate large and ultrafast frequency shifts of the stop bands in the frequency range of second order Bragg diffraction where the photonic band gap is predicted. We compare our results to band structure calculations and deduce a large refractive index change of $\Delta n_{S i}^{\prime} / n_{S i}^{\prime}>2 \%$. We estimate the corresponding dynamic change of the optical density of states to be sufficiently large to allow density of states switching experiments in which the lifetime of internal lights sources is switched.

\subsection{Introduction}

There are several reasons why Si inverse opals are interesting photonic crystals for ultrafast all-optical switching experiments. Firstly, the crystals have a sufficiently large refractive index contrast for a band gap to open up in the range of second order Bragg diffraction [21]. In the region of the gap, switching is expected to lead to ultrafast changes in the density of states [54]. Secondly, their fabrication does not involve complicated lithographic techniques, which has allowed inverse opals to be studied extensively, see Section 1.1.5. The abundance of prior static reflectivity experiments helps us to interpret our new switching data [30, 32-35]. Furthermore, band structure calculations for inverse opals are at hand [16, 130], facilitating the interpretation of the observed stop bands in our spectra. Thirdly, experimental 
control of the dynamics of spontaneous emission from quantum dots inside static photonic crystals was recently demonstrated for the first time with inverse opals [8]. We therefore expect that by controlling the refractive index of $\mathrm{Si}$ inverse opals, the spontaneous emission of light sources inside the crystal can indeed be switched on and off [54]. Finally, calculations of the density of states [54], and recent calculations of the local density of states inside inverse opals (see Ref. [131]) allow us to predict the effect of switching on the emission of light sources inside inverse opals.

A first demonstration of all-optical switching in 3D photonic materials was given by Ref. [90], who reported reflectivity changes in the first order stop band of opals that were infiltrated with Si. Their experiment revealed a serious experimental constraint; due to a short Drude damping time for amorphous Si $\tau_{\text {Drude }}=0.5 \mathrm{fs}$, the maximum feasible refractive index change is limited by the amount of induced absorption. Moreover, since the experiments were performed at probe frequencies above the electronic band gap of $\mathrm{Si}$, the transparency of the unswitched crystal is limited by intrinsic absorption. The first switching experiments in Si inverse opals were reported by Ref. [91], who observed large changes of the transmission in the range of first order Bragg diffraction. The induced absorption in their crystals was strongly reduced by annealing the Si-backbone at a high temperature, causing $\tau_{\text {Drude }}$ to increase drastically from $0.5 \mathrm{fs}$ to $10 \mathrm{fs}$. In the resulting annealed Si inverse opal, large changes in refractive index could be induced, while the absorption remains small.

In this Chapter, we will discuss the first ultrafast switching experiments on inverse opals in the range of second order Bragg diffraction in the vicinity of the expected photonic band gap. Ultrafast switching of Si inverse opal photonic crystal in this frequency range is expected to lead to exciting new physics, in particular to ultrafast changes in the local density of states [54].

\subsection{Sample description}

\subsubsection{Fabrication method}

The Si inverse opal photonic crystal that was used in our experiments was fabricated by the Norris group at the University of Minnesota and further processed by Jeroen Kalkman from the FOM Institute AMOLF in Amsterdam. The sample was made by infiltrating $\mathrm{Si}$ in a silica opaline template: the template was grown on a $\mathrm{Si}$ substrate by a vertical controlled drying method [132]. An ethanolic suspension of pre-shrunk silica spheres with a radius of $r_{\text {sphere }}=469 \mathrm{~nm}$ was used to ensure that the resulting inverse opal has a band gap in the IR region [34]. The resulting silica 
template extends over $5 \times 5 \mathrm{~mm}$, and was infiltrated with Si using chemical vapor deposition at $500{ }^{\circ} \mathrm{C}$. Subsequently, the samples were annealed for one hour at 750 ${ }^{\circ} \mathrm{C}$ in vacuum. During the annealing process, the amorphous $\mathrm{Si}$ in the structure crystallizes into poly-Si, as was confirmed by Raman scattering measurements on a separately deposited thin layer of polycrystalline Si that served as a reference sample [111]. The dielectric constant of polycrystalline Si was also measured on the reference sample and was found to be $\epsilon_{S i}=12.75$ at $\lambda=1530 \mathrm{~nm}$ by transmission measurements $[111,133]$. Finally, the $\mathrm{SiO}_{2}$ template was etched away by a buffered hydrofluoric solution in 8 minutes $\left(6.8 \% \mathrm{HF}, 34.6 \% \mathrm{NH}_{4} \mathrm{~F}, 58.6 \% \mathrm{H}_{2} \mathrm{O}\right)$, resulting in a high-quality air-sphere crystal that is supported by a poly-Si backbone.

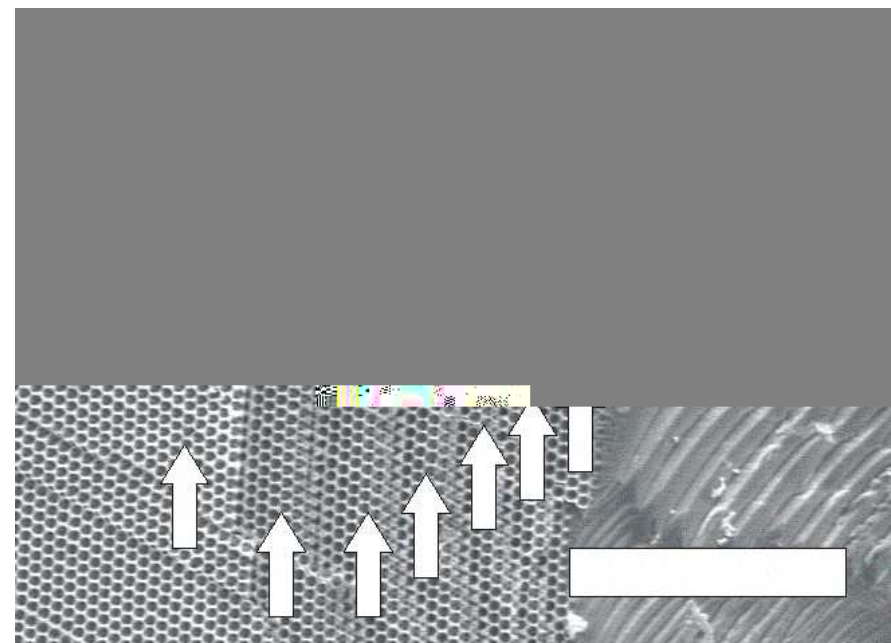

Figure 7.1: Top view SEM image of the Si inverse opal. The scale bar equals $20 \mu \mathrm{m}$. The lattice parameter was measured to be $a=1427 \pm 20 \mathrm{~nm}$ after correcting for tilt [134]. The lattice steps, indicated by arrows, clearly show that the sample consists of 7 layers of air spheres. The lattice structure appears to be fcc. Image courtesy of Jeroen Kalkman.

\subsubsection{Structural analysis}

SEM images of the Si inverse opal crystal, such as the one shown in Fig. 7.1, were analyzed by us to characterize the sample dimensions and quality. The top surface of the crystal corresponds to the $\{111\}$ plane of the fcc-structure. From the SEM image in Fig. 7.1, we determine the thickness of the sample to be 7 layers or $2 \frac{1}{3}$ unit cells, by counting the monolayer steps, indicated by arrows. The typical distance between lattice defects in the crystal was found to exceed $30 \mu \mathrm{m}$, or $\approx 20$ lattice 
periods, indicative of an excellent crystal quality.

In a light scattering experiment, a HeNe laser beam at normal incidence was used to illuminate various positions on the crystal. The resulting diffraction pattern consists of three pairs of diffraction spots in a hexagonal arrangement. The spots correspond to the three grating modes of the crystal, the diffraction angle of each of the spots is related to a symmetry in the crystal's top layer. We made three important observations: firstly, we found that the three diffraction angles vary by less than $5 \%$ with position. Hence, from the grating mode data, we estimate that the in-plane lattice parameter is constant within $5 \%$ over the sample. Secondly, the difference in diffraction angle between the three lattice directions was also less than $5 \%$, indicating that the lattice of the top layer is symmetrical. Finally, the lateral orientation of the lattice was also found to vary by less then $5^{\circ}$ with sample position. We therefore conclude that the top layer of the crystal is indeed hexagonal, and has one preferred lattice orientation.

We have obtained the microscopic structure of our crystals from high resolution scanning electron micrographs, see Fig. 7.2 


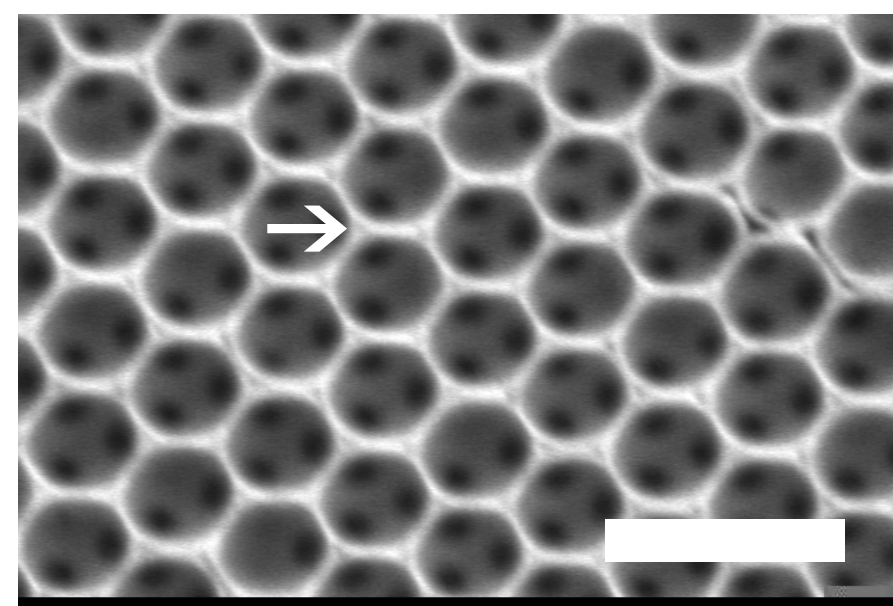

Figure 7.2: High resolution SEM image of the Si inverse opal after HF etching. The scale bar is 2 $\mu \mathrm{m}$. The arrow indicates an almost filled interstice in the structure. From this image we estimate the radius of the windows that interconnect the air spheres to be $r_{c y l}=0.16 \pm 0.05 r_{\text {sphere. }}$. Image courtesy of Jeroen Kalkman.

are can be identified with fcc stop bands by comparing our reflectivity data to band structure calculations for fcc crystals.

\subsection{Linear reflectivity measurements}

\subsubsection{FT-IR reflectivity measurements}

The photonic properties of the crystal were measured by linear reflectivity experiments with a white light Fourier Transform Infra Red (FT-IR) spectrometry setup similar to the one used by Ref. [113]. Broadband, unpolarized light of a halogen lamp was first led through a spectrometer (Biorad). Next, the beam was spatially filtered by a $200 \mu \mathrm{m}$ pinhole, allowing it to be focussed in a $150 \mu \mathrm{m}$ FWHM spot on the sample by a glass objective with numerical aperture $\mathrm{NA}=0.12$. The incident direction was normal to the $\{111\}$ planes of the fcc lattice of the crystal, that correspond to the $\Gamma$-L direction in the band structure of the crystal. The reflected light was detected with a thermo-electrically cooled InAs photodiode that has a broadband spectral response of $2900 \mathrm{~cm}^{-1}$ to $10000 \mathrm{~cm}^{-1}$. All spectra were referenced to that of a gold mirror to obtain an absolute reflectivity calibration. The detection range of the setup is limited by absorption in the glass components for 
frequencies below $4000 \mathrm{~cm}^{-1}$, allowing measurements in the range of second order Bragg diffraction only. Typical reflectivity data taken on two different positions on the sample, are plotted as solid and dashed curves in Fig. 7.3. In all measurements, we identify two clear peaks around $\omega=5200 \mathrm{~cm}^{-1}$ and $\omega=6000 \mathrm{~cm}^{-1}$ with a typical reflectivity of $\approx 60 \%$. The peak at $\omega=5200 \mathrm{~cm}^{-1}$ probably corresponds to the stop gap between the fifth and the sixth band in the band structure shown in Fig. 7.4(b). The peak at $\omega=6000 \mathrm{~cm}^{-1}$ probably corresponds to the stop gap between the eight and ninth band, and is part of the band gap of the crystal. Finally, the peak at $\omega=$ $6600 \mathrm{~cm}^{-1}$ probably corresponds to the stop gap between the tenth and eleventh band. At $7200 \mathrm{~cm}^{-1}$, an additional peak appears that resembles the feature observed in reflectivity from the $\{100\}$ planes of similar Si inverse opals at the same relative frequency $\mathrm{a} / \lambda=1.05$ by Ref. [35]. Possibly, part of our crystal was oriented in the $\{100\}$ direction in the measurement.

We observe a variation in peak reflectivity of all peaks with position on the sample, possibly due to local variations in the density of lattice defects throughout the crystal or by variations in the Si infiltration. The center frequency of the two prominent peaks, as well as the center position of the additional features, however, were found to be independent of position on the sample, which leads to the important conclusion that the crystal lattice is indeed the same everywhere in the sample.

\subsubsection{Laser reflectivity measurements}

The linear reflectivity experiments were reproduced in our pump-probe setup used in the switching experiments (see Chapter 2) with a smaller $\approx 28 \mu \mathrm{m}$ FWHM focus. The polarized, Gaussian output beam of our optical parametric amplifier (Topas OPA) was focussed onto the crystal by a $0.05 \mathrm{NA}$ achromatic lens. The OPA spectra cover the complete stop band in the range of second order Bragg diffraction, since the output frequency of the OPA was scanned over both the idler- $\left(3850-6250 \mathrm{~cm}^{-1}\right)$ and signal range $\left(6250-8620 \mathrm{~cm}^{-1}\right)$. The resulting stitched reflectivity spectra are displayed as symbols in Fig. 7.3. The stitching results in a small discontinuity in the spectra near $\omega=6250 \mathrm{~cm}^{-1}$. The resulting mismatch is indicative of the typical fixed scaling error in the measured OPA reflectance $(\Delta R \approx 5 \%)$. The OPA spectra were taken within $150 \mu \mathrm{m}$ of the same positions A and D as the FT-IR spectra. The OPA reflectivity spectra strongly resemble the FT-IR spectra; all peak positions agree within $\Delta \omega / \omega<2.5 \%$. The good agreement validates a one-to-one comparison between our FT-IR and OPA data.

Next, we study the height of the reflectivity peaks in both the FT-IR and OPA 
experiments. The maximum difference in peak reflectivity between the FT-IR and OPA data is $\triangle \mathrm{R}=15 \%$, which is in agreement with the accumulated scaling errors and the fact that the spectra were taken from slightly different positions on the sample within $\approx 150 \mu \mathrm{m}$. The reflectivity variations with sample position in the OPA data are large, up to $\Delta \mathrm{R}=10 \%$ compared to the variations in the FT-IR spectra, see Fig. 7.3. The larger fluctuations may be related to the small probe focus in the OPA experiments: $\approx 28 \mu \mathrm{m}$ FWHM. Since the FT-IR focus is typically $>150 \mu \mathrm{m}$ FWHM, FT-IR reflectivity data is obtained from several crystal domains simultaneously, resulting in an averaged reflectivity spectrum. The OPA focus, however, is small enough to probe single crystal domains that are typically $30 \mu \mathrm{m}$ in size (see Section 7.2.2). Consequently, OPA spectra are much more sensitive to local variations in the crystal structure than the FT-IR spectra, consistent with the larger reflectivity variations in the OPA data.

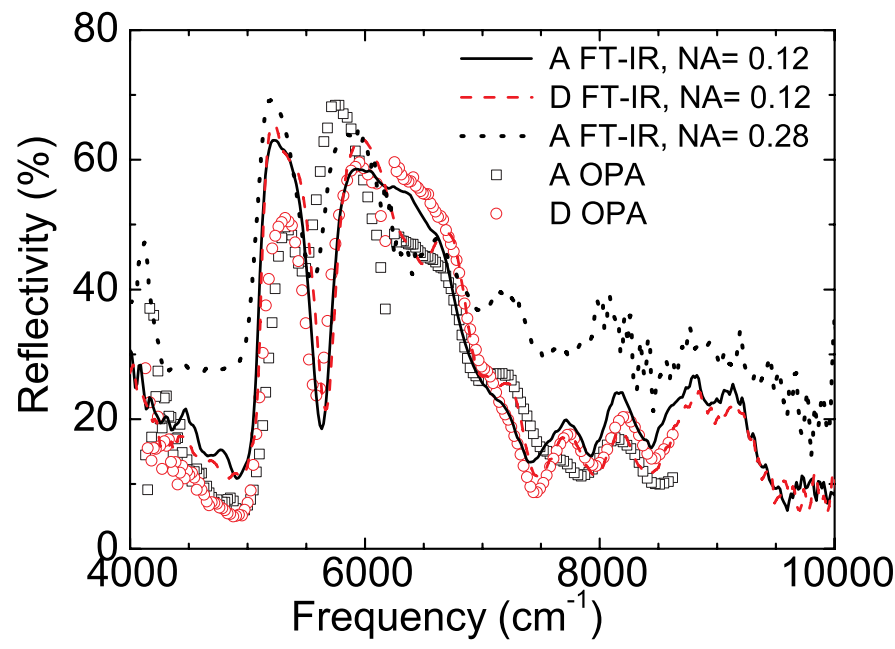

Figure 7.3: Linear reflectivity spectra in the (111) direction of a Si inverse opal at two positions A and $D$ on the sample that are spaced by $1 \mathrm{~mm}$. Solid and dashed curves: measured with an FT-IR setup with a $N A=0.12$ objective lens (focal width $\approx 150 \mu \mathrm{m}$ ). Dotted curve: FT-IR setup with a $N A=0.28$ Cassegrain objective, measured on position $A$ in the center of the sample (focal width $\approx 75$ $\mu m$ ). Symbols: linear Topas OPA reflectivity measurements at the same position on the sample (focal width $\approx 28 \mu \mathrm{m}$.) 


\subsubsection{Extended frequency range interferometry}

To allow a simultaneous measurement of the first- and second order stop bands, the frequency range of the FT-IR reflectivity setup was extended further into the infrared, to $\omega=2900 \mathrm{~cm}^{-1}$ to $10000 \mathrm{~cm}^{-1}$. All absorbing glass optics in the beam path were replaced by non-absorbing reflecting components. Apart from being non-absorbing, an additional advantage of reflecting components is the complete absence of dispersion and chromatic aberrations in such components, which ensures that light of all frequencies is focused on the same spot on the sample. A white light beam (halogen lamp) was spatially filtered by a pair of parabolic mirrors and a $200 \mu \mathrm{m}$ pinhole. A $2 \mu \mathrm{m}$ thin pellicle film with high transmission down to frequencies of $2500 \mathrm{~cm}^{-1}$ was used to replace the IR-absorbing quartz beam splitter. The detection range of the improved setup was limited by the broad spectral response of the InAs photodiode $\left(\omega=2900 \mathrm{~cm}^{-1}\right.$ to $\left.10000 \mathrm{~cm}^{-1}\right)$.

A Cassegrain reflecting objective $(\mathrm{NA}=0.28)$ was used to focus the light onto a spot of $\approx 75 \mu \mathrm{m}$ FWHM the sample. Inside the objective, the light only passes through air, eliminating any IR-absorption or dispersion. A side-effect of the large NA objective is that a considerable part of the diffusive reflectivity is collected by the objective, since it effectively acts as an integrating sphere. This results in an elevated background reflectivity of about 30\%, compared to $5 \%$ in the smallNA experiments, see dashed curve in Fig. 7.3. Similar results are apparent in Ref. [33], but were neither mentioned nor analyzed. Due to the design of the Cassegrain reflecting objective, a light collection cone centered around the optical axis is blocked. The measured spectra are therefore reflectivity spectra integrated between the limiting angles of the objective: $10^{\circ}$ and $16^{\circ}$. Consequently, the reflectivity measurements are done at an effective angle of about $13^{\circ}$, which does not truly correspond to the $\Gamma$ - $\mathrm{L}$ direction in reciprocal space, since the $0^{\circ}$ region is excluded. Nevertheless, we surmise that a comparison is still warranted, since the Bragg condition for the $\{111\}$ planes is blue shifted by only $2.6 \%$, which results in a negligibly small $(\approx 2.6 \%)$ blue shift of the stop band. The center frequency of the observed stop bands will therefore not differ much from the spectra obtained with a glass objective.

The extensive detection range in our reflecting optics setup allows a broadband measurement that spans both the first order peak and the peaks in the region of second order Bragg diffraction (see Fig. 7.4(a). We observe an intense first order reflectivity peak at $\omega=3155 \mathrm{~cm}^{-1}$ with a maximum reflectivity of $80 \%$ and a relative width of $\Delta \omega / \omega=17.7 \%$. The feature near $\omega=4200 \mathrm{~cm}^{-1}$ resembles the feature observed in the reflection from the $\{100\}$ planes at the same relative frequency $a / \lambda=$ 
0.6 by Ref. [35]. Possibly, some crystallites are oriented in the $\{100\}$ direction in the measurement. The additional peaks at frequencies $5211 \mathrm{~cm}^{-1}$ and $5893 \mathrm{~cm}^{-1}$ with peak reflectivities $R=69 \%$ and $R=64 \%$ respectively, were also observed in earlier spectra (see Fig. 7.3). The reflectivity in the second order range is compared to the glass objective data in Fig. 7.3. Both the center positions as well as the maximum reflectivity of the peaks agree well. In Section 7.3.4, the observed peaks are identified with stop bands in the band structure of the fcc crystal.

8

4000

$0 \quad 40 \quad 80$

Figure 7.4: (a) Linear FT-IR reflectivity measurements with a NA=0.28 Cassegrain objective, measured on position $A$ (focal width $\approx 75 \mu \mathrm{m}$ ). The observed peaks in the range of second order Bragg diffraction are labeled I, II, and III. (b) Photonic dispersion relation organized into a band structure for fcc close packed air spheres (radius $r=a / \sqrt{8}$ ) surrounded by spherical Si shells (radius 1.15) connected by cylindrical windows (radius $0.264 r$ ). The volume fraction of solid material is about $\Phi_{S i}=21.3 \%$ $\left(\epsilon_{S i}=12.74\right)$. The frequency scale on panel $(b)$ corresponds to the one on (a) for lattice parameter $a=$ $1427 \mathrm{~nm}$

\subsubsection{Photonic band structure}

To interpret the origin of the peaks observed in Fig. 7.4(a), we have calculated the photonic band structure by a plane-wave expansion method described in detail in Ref. [16]. The model uses the structural constrained input parameters that 
were obtained in Section 7.2. The resulting photonic band structure is plotted in Fig. 7.4(b). We find stop gaps in the $\Gamma$-L direction between bands 2 and 3, bands 5 and 6 , bands 8 and 9 , and bands 10 and 11. The stop gap between bands 8 and 9 is part of the band gap of an infinite perfect crystal [105]. We compare the positions of the calculated stop gaps indicated by gray rectangles in Fig. 7.4(b) to the measured center positions of the peaks in the region of second order Bragg reflection in Fig. 7.4(a). In the scaling of the frequency axis of the measured data, the measured lattice parameter of $a=1427 \mathrm{~nm}$ was used. The good agreement between the measured peak position and the calculated stop gap frequencies in the second order range is striking, particularly since no freely adjustable parameters were used in the calculation.

Nevertheless, we observe two important inconsistencies in the range of first order Bragg diffraction. Firstly, the measured peak appears at $\omega=3199 \mathrm{~cm}^{-1}$ or $\mathrm{a} / \lambda=0.456$, as much as $8 \%$ below the calculated center frequency of the L-gap $\mathrm{a} / \lambda=$ 0.495. We name three possible causes of this large disagreement:

i) the refractive index of Si in the crystal is higher than the value used in the calculations.

ii) the Si filling fraction $\Phi_{S i}$ in the crystal is larger than the value used in the calculations. iii) the lattice parameter normal to the sample interface is larger than the one used in the calculations.

Secondly, the measured width of the first order stop band $\Delta \omega / \omega=17.7 \%$ is smaller than the calculated width $\Delta \omega / \omega=19.5 \%^{1}$. Two possible causes of this mismatch are:

$\left.i^{\prime}\right)$ the refractive index of Si in the crystal is lower than the value used in the calculations.

$\left.i i^{\prime}\right)$ the Si filling fraction $\Phi_{S i}$ in the crystal is smaller than the value used in the calculations.

Strikingly, it appears to be impossible to get both the center frequency as well as the width of the first order peak right, as this would require opposite corrections to either $i$ and $i$ ' or $i i$ and $i i^{\prime}$. We have also tried to get a better agreement for the first order peak by reducing the volume fraction $\Phi_{S i}$ while increasing $n_{S i}$. While this procedure results in a much better agreement for the first order peak, we lose the agreement for the peaks in the range of second order Bragg diffraction. Therefore, the only reason left is $i i i$; the structure may not be cubic close-packed, but slightly rhombohedral. The corresponding band structure will therefore differ from the one for fcc inverse opals shown in Fig. 7.4(b). It is unlikely that reason iii will affect the first order peak, without changing the peaks in the second order region. Therefore, we speculate that there may be other unknown causes for the observed

\footnotetext{
${ }^{1}$ Usually, measured peaks are broader than calculated corresponding stop gaps, notably due to finite size effects and extinction.
} 
mismatch. We exclude Si dispersion as a cause of the mismatch since Si exhibits normal dispersion in this frequency region [96]: the refractive index in the first order range would be reduced, which would cause the calculated stop gap to shift to higher frequencies, increasing the mismatch.

A similar disagreement between reflectivity measurements and band structure calculations between first and second order diffraction in Si inverse opals exists in the data of Ref. [35], who also demonstrates a first order reflectivity peak that is both narrower and red shifted compared to the calculated L-gap. Our calculations are in good agreement with those of Ref. [35]. Their explanation for the effect is that dispersion was not taken into account in the calculations. In the previous paragraph, however, we ruled out this possible reason. Ref. [34] also reported reflectivity spectra of similar Si inverse opals in both first and second order ranges; all peaks agree well with band structure calculations. In this case, however, the measurements in the first and second order frequency ranges originate from two separate samples with different lattice parameters, and thus potentially different structural properties. In conclusion, there is still no definitive explanation for the inconsistency between band structure calculations and reflectivity experiments for Si inverse opals. We propose that the mismatch may be caused by a slightly rhombohedral unit cell, which will result in a modified band structure. A distortion of the fcc structure is consistent with other reports that vertical controlled drying yields opal structures that deviate from fcc [37].

Nevertheless, in the frequency range of second order Bragg diffraction, where our switching measurements have taken place, we find good agreement between our model and measurements without free parameters. We therefore neglect the $8 \%$ frequency mismatch of the first order peak. In the next Section 7.4, we will compare our experimental data to band structure calculations to quantify the amount of switching in our crystals.

\subsection{Pump-probe reflectivity measurements}

\subsubsection{Differential reflectivity}

Ultrafast switching experiments with our independently tunable OPAs were carried out on the Si inverse opal crystals described in Section 7.2. The reflectivity data covers the complete range of second order Bragg diffraction in our sample. The measured spectra consist of multiple peaks that were identified with stop gaps in Fig. 7.4. The reflectivity was studied as a function of probe delay $\tau$ over a 1800 $\mathrm{cm}^{-1}$ broad probe frequency range $\omega_{\text {probe }}$. A two-photon process was used to ex- 
cite carriers in order to maximize the homogeneity of the switched crystal. The pump frequency that was chosen depends on the probe frequency range, to allow polarization based separation of pump and probe light (see Chapter 2). At low probe frequencies $\omega_{\text {probe }}<6250 \mathrm{~cm}^{-1}$, a high pump frequency $\omega_{\text {pump }}=6450 \mathrm{~cm}^{-1}$ was chosen. For probe frequencies $\omega_{\text {probe }}>6250 \mathrm{~cm}^{-1}$, the pump frequency was reduced to $\omega_{\text {pump }}=5000 \mathrm{~cm}^{-1}$, below the red edge of stop band I. In all experiments, the probe irradiance was kept at least 10 times lower than the pump irradiance to avoid artifacts due to self modulation of the probe light. In the experiment shown in Fig. 7.5, the pump irradiance was set to $I_{0}=25 \pm 3 \mathrm{GWcm}^{-2}$ for measurements on stop band III with $\omega_{\text {pump }}=5000 \mathrm{~cm}^{-1}$, and to $I_{0}=4 \pm 1 \mathrm{GWcm}^{-2}$ for experiments on stop bands I and II where $\omega_{\text {pump }}=6450 \mathrm{~cm}^{-1}$. Since the density of excited carriers $N_{\text {eh }}$ is proportional to $I_{0}^{2} \beta / \omega_{\text {pump }}$ (see Appendix A) we can estimate the ratio of excited carrier density on both frequency ranges. Using the dependence of $\beta$ on pump irradiance (Appendix C), we deduce that the expected carrier density for the data on stop bands I and II is about $12 \pm 4$ times larger than the carrier density for the experiments on the stop band III.

The resulting differential reflectivity of the crystal $\Delta R / R\left(\tau, \omega_{\text {probe }}\right)$ at ultrafast time scales is represented as a three-dimensional surface plot in Fig. 7.5. Near stop bands I, II and III, we observe clear dispersive shapes in the differential reflectivity, caused by a shift of the peaks towards higher frequency. At the low frequency edge of stop band I around $5260 \mathrm{~cm}^{-1}$, the reflectivity displays a large and ultrafast decrease $\Delta R / R=-40 \%$ within 500 fs. At $\omega=5893 \mathrm{~cm}^{-1}$, reflectivity of stop band II decreases by $\Delta R / R=-20 \%$, due to a combination of a shift combined with some induced absorption in the sample. At frequencies above stop band III $\omega>7000$ $\mathrm{cm}^{-1}$, we observe additional dispersive curves that are related to the large shift of the reflectivity features above the stop band. The observed strong variations provide evidence of a large change in refractive index. Unlike in the experiments with woodpile structures (see Chapter 6), we do not observe a Kerr effect at zero time delay.

\subsubsection{Switched spectra at fixed probe delay}

The effect of the excited free carriers on the stop band is studied in more detail by measuring the reflectivity changes at a fixed time delay of $\tau \approx 1$ ps. Fig. 7.6 (upper panel) shows measurements of the pumped and unpumped reflectivity in the range of second order Bragg diffraction of the sample at normal incidence. Due to dispersion in the probe path of the setup, there is a $<500 \mathrm{fs}$ variation of the delay time with frequency. The pump irradiance was set to $I_{0}=24 \pm 2 \mathrm{GWcm}^{-2}$ for 
Figure 7.5: Differential reflectivity as a function of both probe frequency and probe delay. The pump frequency and peak irradiance were $\omega_{\text {pump }}=6450 \mathrm{~cm}^{-1}$ and $I_{0}=4 \pm 1 \mathrm{GWcm}^{-2}$ on the red part, and $\omega_{\text {pump }}=5000 \mathrm{~cm}^{-1}$ and $I_{0}=25 \pm 3 \mathrm{GWcm}^{-2}$ on the blue part of the spectrum. The probe delay was varied in small steps of $\Delta t=50 \mathrm{fs}$ on the blue edge and in steps of $\Delta t=500 \mathrm{fs}$ at the red edge. The probe wavelength was tuned from 1600 to $2100 \mathrm{~nm}$ in $\Delta \lambda=10 \mathrm{~nm}$ steps in the low frequency range, and in $5 \mathrm{~nm}$ steps in the high-frequency range.

measurements on the blue part of the stop band with $\omega_{\text {pump }}=5000 \mathrm{~cm}^{-1}$, and $I_{0}=$ $11 \pm 2 \mathrm{GWcm}^{-2}$ on the red part of the stop band with $\omega_{\text {pump }}=6450 \mathrm{~cm}^{-1}$. Using the analysis in Section 7.4.1, we estimate that the excited carrier density $N_{e h}$ on the red and blue parts in Fig. 7.6 is roughly equal; the ratio is less than $1.5 \pm 0.4$. Since the difference in carrier density for both data sets is relatively small, Fig. 7.6 can be used to compare the effect of excited carriers on both stop band edges.

The unpumped spectrum in Fig. 7.6 (upper panel) demonstrates a broad stop band with a relative width $\Delta \omega / \omega=28 \%$ FWHM that is centered around $\omega=5930$ $\mathrm{cm}^{-1}$. The stop band consists of two sub peaks at frequencies $\omega=5320 \mathrm{~cm}^{-1}$ with maximum reflectivity $R=51 \%$ and $\omega=5950 \mathrm{~cm}^{-1}$ with maximum reflectivity $\mathrm{R}=$ $60 \%$. The data are in good agreement with the FT-IR reflectivity measurements that are plotted as a dashed line in the same Figure. 
The switched reflectivity spectra on the same sample are shown as closed circles in Fig. 7.6 (upper panel). The entire stop band has shifter towards higher frequency, indicating that the average refractive index of the crystal has been reduced. The stop band has shifted to higher frequency by as much as $\Delta \omega / \omega=1.5 \%$ at the red edge, and by $\Delta \omega / \omega=0.7 \%$ at the blue edge of the stop band. The shift of the feature above the stop band at $7700 \mathrm{~cm}^{-1}(\Delta \omega / \omega=1.2 \%)$ will be used in the analysis in Section 7.4.5 to compare the experimental shift to band structure calculations. Importantly, both the low and high frequency edge of the stop band have shifted, indicating the absence of separate dielectric and air bands that were present in the Bragg stack experiments in Chapter 5. The observed shift of the entire stop band is consistent with predictions by Ref. [54].

The changes in reflectivity are even more pronounced in the differential reflectivity of the sample that is plotted in Fig. 7.6 (lower panel). On the red edge of stop band I, at $\omega=5110 \mathrm{~cm}^{-1}$, we observe a large decrease in the reflectivity by $\Delta \mathrm{R} / \mathrm{R}=-50 \%$. On the blue edge of stop band III, at $\omega=7020 \mathrm{~cm}^{-1}$ the differential reflectivity amounts to $\Delta \mathrm{R} / \mathrm{R}=+40 \%$. In between stop band I and II, and close to the features above stop band III in the range $\omega=7000-9000 \mathrm{~cm}^{-1}$, the frequency shift causes dispersive curves that were already observed in Fig. 7.5. In conclusion, the excitation of free carriers in the backbone of the crystal causes a large blue shift of up to $1.5 \%$ of all 3 stop bands in the range of second order Bragg diffraction as well as of features above this range.

\subsubsection{Switching time traces}

The large and ultrafast shift of the stop band with time is studied in more detail in Fig. 7.7. We have measured the frequency position of the red edge of the stop band at $\omega=5045 \mathrm{~cm}^{-1}$, at a large range of delay times after excitation. The pump parameters are: $\omega_{\text {pump }}=6450 \mathrm{~cm}^{-1}, I_{0}=4 \pm 1 \mathrm{GWcm}^{-2}$. From each spectrum, the frequency position of the red edge of the stop band was determined at the foot of the peak at $\mathrm{R}=15 \%$ (indicated by the arrow in Fig. 7.6 (upper panel)), to minimize the contribution of induced absorption to the observed shift. The relative frequency shift $\Delta \omega / \omega$ is plotted versus probe delay in Fig. 7.7. We observe a large shift of the stop band edge from 0 to $\Delta \omega / \omega=1.1 \%$ within 500 fs. The effect subsequently decreases exponentially with a decay time of $\tau=21 \pm 4$ ps (least squares fit) to a small residual shift $\Delta \omega / \omega=0.1 \%$. The short relaxation time is in good agreement with the typical carrier relaxation time of 18 ps that we found in poly-Si woodpile samples studied in Chapter 6. The decay times are much faster than carrier relaxation times in bulk $\mathrm{Si}$, since our photonic crystals are made of poly crystalline silicon, 


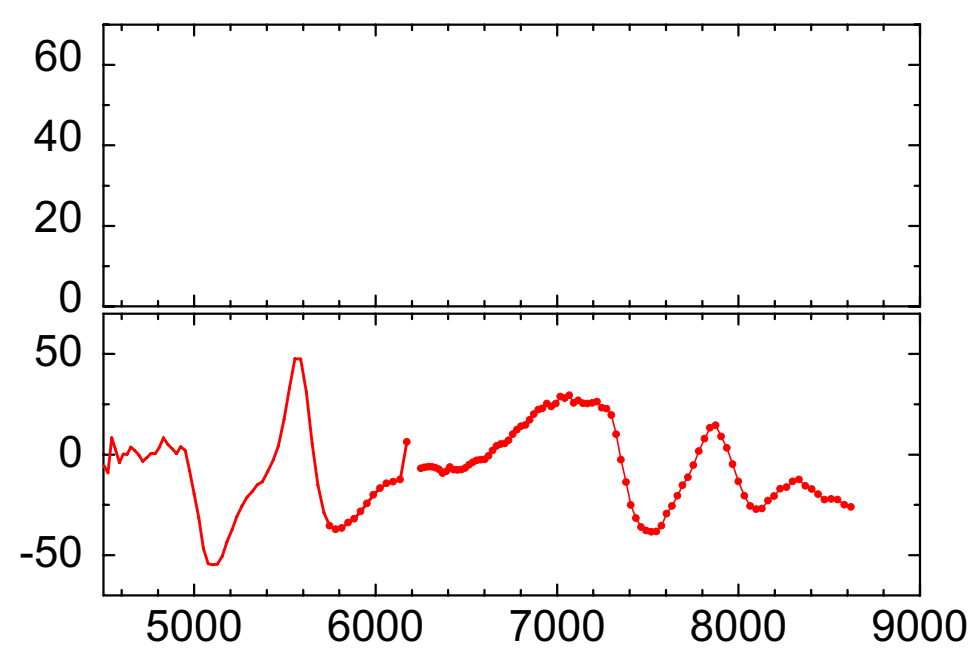

Figure 7.6: (upper panel) Unswitched (open triangles) and switched (closed circles) reflectivity spectra of the sample at normal incidence. In experiments on the red part of the spectrum (4500 $\left.\mathrm{cm}^{-1}<\omega_{\text {probe }}<6250 \mathrm{~cm}^{-1}\right)$, the pump frequency was chosen to be $\omega_{\text {pump }}=6450 \mathrm{~cm}^{-1}$. In experiments on the blue edge $\left(6250 \mathrm{~cm}^{-1}<\omega_{\text {probe }}<8620 \mathrm{~cm}^{-1}\right)$, the pump frequency was lowered to $\omega_{\text {pump }}=$ $5000 \mathrm{~cm}^{-1}$. The pump irradiance for the switched spectrum was $I_{0}=11 \pm 2 \mathrm{GWcm}^{-2}$ on the red part and $I_{0}=24 \pm 2 \mathrm{GWcm}^{-2}$ on the blue part of the stop band. The switched spectra were measured at a pump-probe time delay of $\approx 1 \mathrm{ps}$. We observe a large blue shift of up to $1.5 \%$ of the complete stop band in the range of second order Bragg diffraction as well as off features outside the stop band. The dashed blue line is a FT-IR reflectivity spectrum that was measured on the same position on the sample (within $100 \mu \mathrm{m}$ ). (lower panel) Differential reflectivity spectrum. A blue shift of the peak at $\omega=5200 \mathrm{~cm}^{-1}$ is illustrated by strong decreases and increases of the reflectivity below and above this frequency respectively. Similar variations with frequency appear at frequencies above $7000 \mathrm{~cm}^{-1}$, and are related to a blue shift of the reflectivity features in that frequency range.

whose lattice defects and grain boundaries act as efficient carrier recombination traps [110]. The relatively fast decay time implies that switching could potentially be repeated at a rate above $25 \mathrm{GHz}$, which is relevant to possible future switching and modulation applications.

\subsubsection{Induced probe absorption}

Besides a frequency shift which is related to a change in the real part of the refractive index, we also observe the effects of induced absorption, which are related to an 


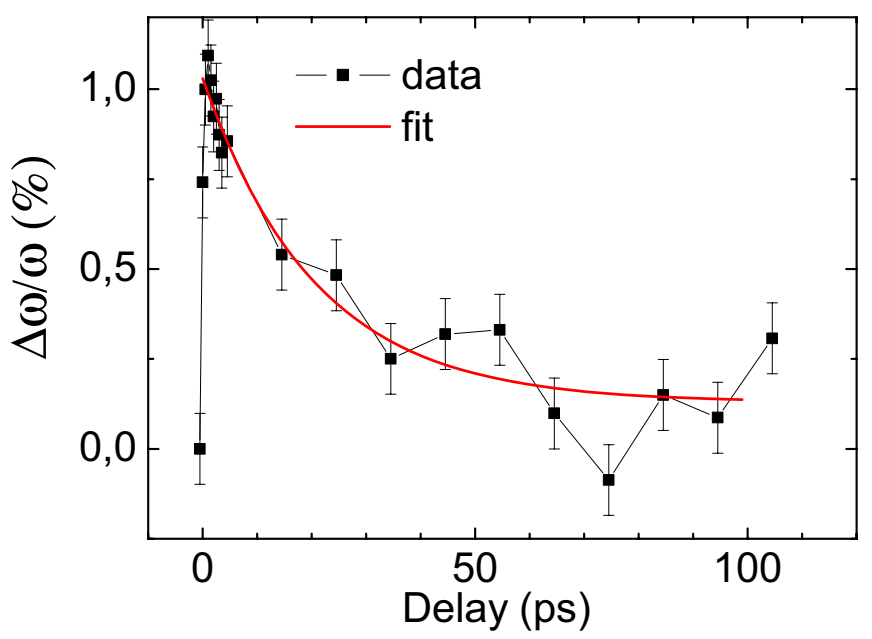

Figure 7.7: Blue shift of the red edge of the stop band at $\omega=5075 \mathrm{~cm}^{-1}$ plotted versus probe delay (symbols). The pump frequency and irradiance were $\omega=6450 \mathrm{~cm}^{-2}$ and $4 \pm 1 \mathrm{GWcm}^{-2}$ respectively. The large shift amounts to $\Delta \omega / \omega=1.1 \%$ within the first $500 \mathrm{fs}$. The subsequent exponentially decay is well fitted with a single exponential decay $\Delta \omega / \omega=A+\exp (-t / \tau)$ (red curve), where the decay time $\tau=21 \pm 4 \mathrm{ps}$, and the small offset of $\Delta \omega / \omega$ is $A=0.13 \%$.

increase in $n_{S i}^{\prime \prime}$. The induced absorption manifests itself in a reduction of the reflectivity maximum after excitation. We therefore plot in Fig. 7.8 the relative decrease in reflectivity maximum of stop band II at frequency $\omega=5882 \mathrm{~cm}^{-1}$ as a function of delay time. The data were measured in the same run as the experiment shown in Fig. 7.7. The maximum decrease in reflectivity of the stop band directly after excitation, is $\Delta R / R \approx-21 \%$. Note that this decrease is not only due to absorption but also to a shift. In Chapter (6), the reduction in peak reflectivity of the woodpile samples was related to the induced absorption through exact model method calculations shown in Fig. 6.15. In absence of a stable real space model for Si inverse opals, we estimate the amount of induced absorption by comparing the observed $\Delta R / R=$ $-21 \%$ to the calculated decrease in the Si woodpiles, see Fig. 6.15 . We estimate that the upper bound of the induced absorption in the Si backbone of the inverse opal crystal is $n_{S i}^{\prime \prime}<0.1$. Using the Si volume fraction $\Phi_{S i}=21.3 \%$, obtained in 7.2.2, we estimate the resulting probe absorption length in our switched inverse opal to be $\ell_{a b s}>\lambda / 4 \pi \Phi_{S i} n_{S i}^{\prime \prime}=6.3 \mu \mathrm{m}$, comparable to the thickness of the sample $\mathrm{L}=7 \times d_{\{111\}}=$ $5.7 \mu \mathrm{m}$. We conclude that for refractive index changes larger than $2 \%$, the sample may lose its transparency. Fortunately, smaller changes in the refractive index will suffice to considerably change the density of states (see Section 7.5.3), and thus less 
absorption will occur.

Fig. 7.8 also shows how the reflectivity change evolves in time after the initial decrease to $\Delta R / R=-21 \%$. The effect decays exponentially to $\Delta R / R=-10 \%$ with a decay time of $4 \pm 1$ ps, followed by a much slower decay with about ns decay times. The combination of a fast and a much slower decay indicates that there are two separate relaxation processes involved in our sample. The fast relaxation process is likely related to the fast recombination of carriers in the poly-Si backbone of the crystal that was discussed in the previous Section. It is presently unclear why the induced absorption decays about five times faster than the induced stop band shift shown in Fig. 7.6, which was obtained from the same set of spectra. The subsequent, much slower recombination process at ns times may be related to recombination of excited carriers in the underlaying Si-wafer substrate. In bulk $\mathrm{Si}$ at the carrier densities in the range of our experiment $\left(10^{19} \mathrm{~cm}^{-3}\right)$, carrier recombination is dominated by Auger effects, with recombination times of the order of $10 \mathrm{~ns}$ [135]. Any change in the substrate is likely to change the magnitude of the reflectivity of the whole sample, while it should not affect the frequency position of the stop band, which is related to changes in the poly-Si material in the photonic crystal only. This hypothesis is consistent with our data, since we find that the slowly decaying reflectivity decrease in Fig. 7.8 is still large after 100 ps, while the residual effect on the stop band shift in Fig. 7.7 is almost negligible.

\subsubsection{Irradiance scaling}

To verify that the observed shifts are caused by free carriers generated by twophoton absorption, we study how the observed switching effects scale with pump irradiance. Switched reflectivity spectra were taken at position A on the sample. The shift of the red edge at $\omega=5075 \mathrm{~cm}^{-1}$ was determined at a reflectivity level $\mathrm{R}=20 \%$ at 1 ps after excitation. The pump frequency was $\omega_{\text {pump }}=6450 \mathrm{~cm}^{-1}$. We plot the measured frequency shift versus irradiance squared as symbols in Fig. 7.9. The measured shift is proportional to the pump irradiance squared up to $I_{\text {pump }}^{2}=$ $300 \mathrm{GW}^{2} \mathrm{~cm}^{-4}$. The data point at high pump irradiance $I_{\text {pump }}^{2}=520 \mathrm{GW}^{2} \mathrm{~cm}^{-4}$, lies below the line, likely due to induced absorption. The maximum observed shift of the stop band is as much as $\Delta \omega / \omega=2.8 \%$ at $I_{0}=21 \mathrm{GWcm}^{-2}$.

We have also measured the shift of the small reflectivity peak that appears just above the blue edge of stop band III at $\omega=7742 \mathrm{~cm}^{-1}$ at 1 ps after excitation. The pump frequency was $\omega_{\text {pump }}=5000 \mathrm{~cm}^{-1}$. Data of frequency shift versus irradiance squared is shown as symbols in Fig. 7.10. The measured shift is proportional to the pump irradiance squared up to $I_{\text {pump }}^{2}=400 \mathrm{GW}^{2} \mathrm{~cm}^{-4}$. The maximum observed 


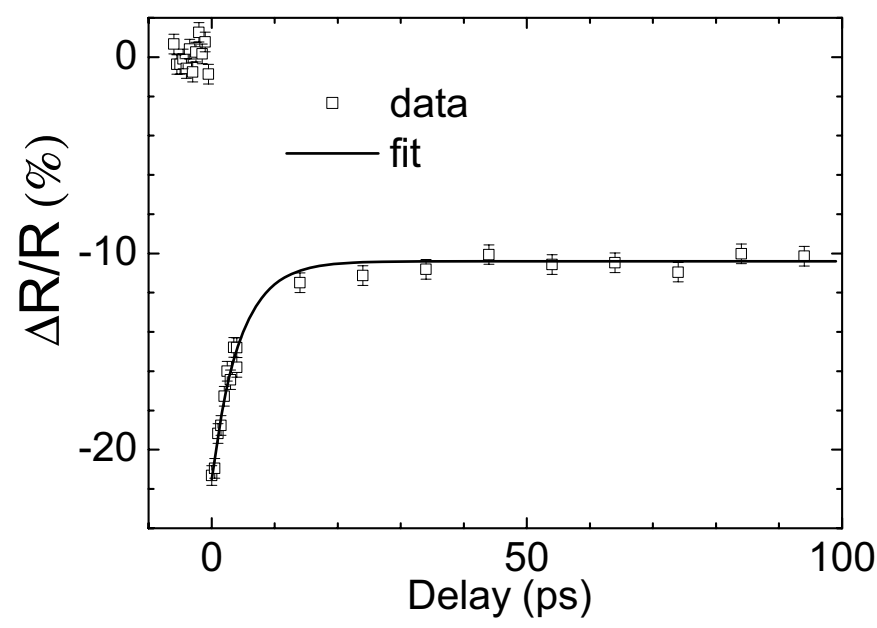

Figure 7.8: Differential reflectivity decrease at $\omega=5882 \mathrm{~cm}^{-1}$ versus probe delay. The pump frequency and irradiance were $\omega=6450 \mathrm{~cm}^{-2}$ and $11 \pm 2 \mathrm{GWcm}^{-2}$ respectively. The large decrease amounts to $\triangle R / R=-21 \%$ within the first $500 f s$, followed by an exponentially decay that is well fitted with a single exponential decay $\Delta \omega / \omega=A+B \exp (-t / \tau)$ (curve), with amplitude $B=-11 \%$, decay time $\tau=4.5 \pm 0.5 \mathrm{ps}$, the offset of $\Delta R / R$ is $A=-10 \%$. The remaining offset in $\Delta R / R: A=-10 \%$ appears to decay slowly at ns times and is attributed to the substrate.

shift in the linear part of the curve at $I_{0}=18 \mathrm{GWcm}^{-2}$ is $\Delta \omega / \omega=1.1 \%$, about three times lower than the maximum shift of the red edge.

Both at the low frequency edge, as well as at high frequencies, the shift of the stop band scales linearly with the pump irradiance squared. From this we conclude that a two-photon process is the dominant excitation mechanism in our experiments. The induced effects remain after the pump pulse, and decay at a time scale in between the decay time for free carriers in amorphous- [128] and single crystalline Si [135]. We therefore conclude that the changes are indeed caused my free carriers. The induced free-carrier effects are also consistent with Drude dispersion since the changes are smaller at higher frequencies.

\subsubsection{Comparison to band structure}

We want to relate the measured frequency shifts shown in Fig. 7.9 and 7.10 to a refractive index change in the Si backbone of our crystals. For that reason, we have performed band structure calculations similar to the one shown in Fig. 7.4. The refractive index in the calculations was varied to emulate our experiment in a quasi- 


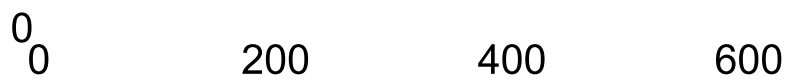

Figure 7.9: (symbols) Measured shift of the red edge of stop band I versus pump irradiance squared measured at a fixed reflectivity of $R=15 \%$ at 1 ps after excitation. The pump frequency was $\omega=$ $6450 \mathrm{~cm}^{-2}$. The quadratic scaling with irradiance confirms a two-photon excitation process. (curve) Calculated shift of the upper edge of the fifth band in the $\Gamma$-L direction in the band structure in Fig. 7.4 plotted versus $\Delta n_{S i}^{\prime} / n_{S i}^{\prime}$. The upper $x$ axis was scaled such that the theory coincides with the measured data, directly relating the pump irradiance to the refractive index change.

static approximation. The red edge of stop band I in the $\Gamma$-L direction corresponds to the fifth band in the band structure shown in Fig. 7.4(b). The calculated shift of the upper edge of the fifth band is plotted in Fig. 7.9 as a function of $\Delta n_{S i}^{\prime} / n_{S i}^{\prime}$ (upper scale) in the same graph as the measured shift of the red edge of stop band I. Note that the calculated data shows that in the range of our experiment, the frequency shift of the red edge of the stop band scales linearly with $\Delta n_{S i}^{\prime} / n_{S i}^{\prime}$ up to $-2.0 \%$. To compare the calculated and measured frequency shifts, we set both $\Delta \omega / \omega$ axes to the same scale, and varied the scale of $\Delta n_{S i}^{\prime} / n_{S i}^{\prime}$ until the calculated curve matches the measured data. The upper x-axis now directly relates a measured shift to a refractive index change. At $\omega_{\text {pump }}=6450 \mathrm{~cm}^{-1}, I_{0}=14 \mathrm{GWcm}^{-2}$ for instance, we find that the refractive index at the red edge $\left(\omega=5075 \mathrm{~cm}^{-1}\right)$ changes by as much as $\Delta n_{S i}^{\prime} / n_{S i}^{\prime}=-2.0 \%$.

We follow a similar procedure to quantify the refractive index change at the blue edge of the stop band. Although the shift of the feature at $\omega=7742 \mathrm{~cm}^{-1}$ in Fig. 7.10 does not directly correspond to a stop band in the band structure, we compare 


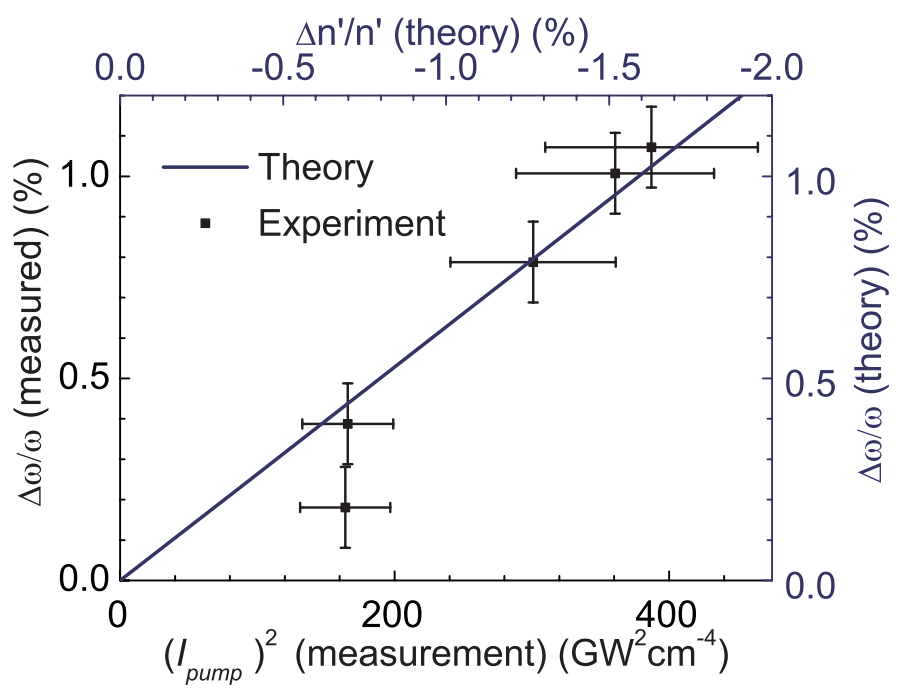

Figure 7.10: (symbols) Measured shift of the small reflectivity peak at $\omega=7742 \mathrm{~cm}^{-1}$ at 1 ps after excitation versus pump irradiance squared. The pump frequency was $\omega=5000 \mathrm{~cm}^{-1}$. The quadratic scaling with irradiance confirms a two-photon process. (curve) Calculated shift of the upper edge of the 11 th band in the band structure in Fig. 7.4 in the $\Gamma$-L direction plotted versus $\Delta n_{S i}^{\prime} / n_{S i}^{\prime}$. The upper $x$ axis was scaled such the theory coincides with the measured data, directly relating the pump irradiance to the refractive index change.

the shift of the eleventh band in the band structure (see Fig. 7.4), which is located at roughly the same frequency. Again, the top abscissa in Fig. 7.10 is adjusted to obtain a good overlap between experiment and theory. From the resulting plot in Fig. 7.10, we deduce a large maximum refractive index change $\Delta n_{S i}^{\prime} / n_{S i}^{\prime}=-1.6 \%$ at $\omega_{\text {pump }}=5000 \mathrm{~cm}^{-1}, \omega=7742 \mathrm{~cm}^{-1}$, and $I_{0}=20 \mathrm{GWcm}^{-2}$. We surmise that the observed refractive index changes are sufficiently large to switch the density of states on ultra fast timescales [54] (see Section 7.5).

\subsection{Discussion}

In this Section, several aspects of our switching experiments are discussed in more detail. We use the Drude model to obtain the induced carrier density $N_{e h}$. From $N_{e h}$ and the pump irradiance $I_{0}$, we deduce the two-photon absorption coefficient $\beta$. Finally, we discuss how the induced change in the refractive index would affect the photonic density of states inside an infinitely large crystal. 


\subsubsection{Carrier density}

From our observations, we have concluded that the ultrafast refractive index changes are caused by free carriers. The optical response of such carriers appears to be well described by the Drude model [3, 73, 94, 136] (see also Appendix A). Therefore we use the Drude model to relate the deduced refractive index changes $\Delta n_{S i}^{\prime} / n_{S i}^{\prime}$ to a carrier density in the Si backbone of the crystal. For silicon, at carrier densities below $10^{20} \mathrm{~cm}^{-3}$, the refractive index is linear with the carrier density within $0.2 \%$ (see Appendix A):

$$
n^{\prime}\left(\omega, N_{e h}\right)=\sqrt{\epsilon_{B}}-\frac{1}{2 \sqrt{\epsilon_{B}}}\left(\frac{\omega_{p}}{\omega}\right)^{2}=\sqrt{\epsilon_{B}}-\frac{e^{2}}{2 \sqrt{\epsilon_{B}} \epsilon_{0} m_{o p t}^{*} m_{e} \omega^{2}} N_{\mathrm{eh}},
$$

where $\omega_{p}=\sqrt{N_{\mathrm{eh}} e^{2} / \epsilon_{0} m_{o p t^{*}} m_{e}}$ is the plasma frequency. We find that the observed change $\Delta n_{S i}^{\prime} / n_{S i}^{\prime}=-2.0 \%$ at probe frequency $\omega=5075 \mathrm{~cm}^{-1}$ corresponds to a plasma frequency $\omega_{p}=0.741 \omega=3623 \mathrm{~cm}^{-1}$. By using an optical effective mass $m_{o p t}^{*}=0.15$, see Ref. [73], we deduce the corresponding carrier density to be $N_{e h}=2.1 \times 10^{19} \mathrm{~cm}^{-3}$. The smaller refractive index change $\Delta n_{S i}^{\prime} / n_{S i}^{\prime}=-1.6 \%$ in the experiment above stop band III at $\omega=7742 \mathrm{~cm}^{-1}$ corresponds to a somewhat higher plasma frequency $\omega_{p}=$ $0.639 \omega=4943 \mathrm{~cm}^{-1}$. The resulting carrier density at this frequency is $N_{e h}=4.1 \times 10^{19}$ $\mathrm{cm}^{-3}$. In both analyzed cases, we see that excellent switching conditions appear if the probe frequency $\omega$ is about 1.5 to 2 times the plasma frequency of the carriers, as predicted in Section 2.2.3.

\subsubsection{Two-photon absorption coefficient}

The two-photon absorption coefficient $\beta$ is deduced by relating the carrier density $N_{e h}$ to the pump intensities used in the experiments. For a two-photon excitation process we find that this relation is equal to:

$$
N_{\text {eh }}=\frac{I_{0}^{2} \tau_{\text {pump }}}{2 \hbar \omega_{\text {pump }}} \beta,
$$

and thus the two-photon absorption coefficient equals:

$$
\beta=\frac{2 \hbar \omega_{\text {pump }} N_{\text {eh }}}{I_{0}^{2} \tau_{\text {pump }}} .
$$

At a pump frequency $\omega_{\text {pump }}=6450 \mathrm{~cm}^{-1}$ we deduced the carrier density to be $N_{e h}=2.1 \times 10^{19} \mathrm{~cm}^{-3}$ for a pump irradiance $I_{0}=14 \mathrm{GWcm}^{-2}$. The corresponding two-photon absorption coefficient is $\beta=230 \pm 100 \mathrm{cmGW}^{-1}$. In the experiments 
where the pump frequency is $\omega_{\text {pump }}=5000 \mathrm{~cm}^{-1}$, we find a somewhat lower twophoton absorption coefficient of $\beta=170 \pm 100 \mathrm{cmGW}^{-1}$. The value of $\beta$ decreases with pump frequency, in agreement with the data in Appendix $C$. The absolute values of $\beta$ that we deduced, however, are at least two orders of magnitude larger than the value found by us in z-scan measurements in bulk Si (see Appendix C). The reason for this mismatch in $\beta$ is presently unclear. We can exclude the variation of the optical effective mass $m_{o p t}^{*}$ with carrier density, since $m_{o p t}^{*}$ varies by at most factor of two in the range of our experiments $N_{\text {eh }}<10^{20}$ [103]. We conclude that a better understanding of the free-carrier generation process in our Si inverse opals requires an independent measurement of the two-photon absorption coefficient, for instance by z-scan measurements on poly-Si reference samples (see Appendix C).

\subsubsection{Switched density of states}

Here, we investigate if the refractive index changes that were demonstrated in this Chapter are sufficiently large to observe changes in the photonic density of states (DOS) inside our crystals. To calculate the density of states we have modeled our structure in the same way as in the band structure calculations in Fig. 7.4(b). A detailed description of the DOS calculations can be found in Refs. [16, 54]. We have based our model on the structural data obtained from SEM images in Section 7.2.2. Absorption and dispersion in the Si backbone were not taken into account. We have calculated the DOS per unit volume for the unswitched crystal using a Si refractive index comparable to the one in our unswitched crystal: $n_{S i}^{\prime}=3.57$ (solid curve in Fig. 7.11). The crystal appears to have a $6.0 \%$ wide band gap where the DOS vanishes for frequencies between $0.818<\omega a / 2 \pi c<0.868$.

The dynamic behavior of the DOS in the switched crystals was studied in a quasi static way by reducing the refractive index of the backbone by the same amount as observed in our experiments: by $\Delta n_{S i}^{\prime} / n_{S i}^{\prime}=-2.0 \%$ to $n_{S i}^{\prime}=3.50$ (see dashed curve in Fig. 7.11). We compare our results to the DOS for a homogeneous reference sample with effective refractive index $n_{\text {eff }}=1.87$, equal to that in the unswitched crystal $^{2}$ (dashed curve). The analytical result for the DOS in a homogeneous medium is $\omega^{2} n_{\text {eff }}^{3} /\left(\pi^{2} c^{3}\right)$, which may be rewritten in terms of normalized frequency $\hat{\omega}=\omega / \omega_{a}=\omega a / 2 \pi c$ as $n_{\text {eff }}^{3} \hat{\omega}^{2} \times\left[\omega_{a}^{2} /\left(\pi^{2} c^{3}\right)\right][16]$.

We observe three important effects in the calculated switched DOS, which were also predicted by Ref. [54]. Firstly, the photonic band gap becomes narrower:

\footnotetext{
${ }^{2}$ The effective refractive index was obtained from the volume averaged dielectric constant $n_{e f f}=\sqrt{\bar{\epsilon}}$, which holds for photonic crystal in the low frequency limit [137].
} 
$\Delta \omega / \omega=5.4 \%(0.832<\omega a / 2 \pi c<0.878)$, consistent with a reduced refractive index contrast. Secondly, we observe a shift of all spectral features, including the band gap, to higher frequencies. The magnitude of the calculated shift of the band gap center frequency is $1.4 \%$, comparable to the shift that was observed in reflectivity features shown in Fig. 7.6. We must note, however, that a direct comparison between directional reflectivity spectra and the DOS, which is a wave vector averaged property of the crystal, is not warranted. At any rate, the DOS features shift linearly with $\Delta n_{S i}^{\prime} / n_{S i}^{\prime}$, resembling the shift of the reflectivity features in Fig. 7.9. Finally, DOS calculations demonstrate that switching causes large dispersive changes in the DOS in the frequency range of the band gap. At $\omega a / 2 \pi c=0.80$, the DOS increases by $133 \%$ of the DOS in the reference, at $\omega a / 2 \pi c=0.91$, we observe a large decrease in the DOS by $-220 \%$ of the DOS in the reference sample. At a frequency $\omega a / 2 \pi c=$ 0.817, which is just inside the band gap of the unswitched crystal, the DOS changes from 0 to $68 \%$ of the DOS in the reference sample, which would allow the emission rate of an light source to be switched from the "off" state to the "on" state.

Interestingly, the magnitude of the refractive index change required to induce sizable changes in the emission rate of sources in our analysis is about 5 times smaller than proposed in the switching scheme of Ref. [54], in which the band gap of a GaAs inverse opal is shifted by its band width. Moreover, the large, $\approx 10 \%$ refractive index change that was proposed by Ref. [54] will bring about a large amount of absorption, that will strongly reduce the probe absorption length. Finally, the more advanced switching scheme of Ref. [54], which uses 2 subsequent pump pulses to switch the DOS up and down, can be replaced by a single pulse experiments, which induces both Kerr and free-carrier effects see Chapter 6.

The emission rate of light sources is proportional to the local radiative density of states [138] (LDOS). Recent reports by Ref. [131] have revealed that in inverse opals, the LDOS strongly depends on both the dipole orientation, as well as on the position of the emitters. The DOS/vol. shown in Fig. 7.11 is the unit cell averaged LDOS. We now assume that changes in the LDOS at the position of the emitters are proportional to the calculated, sizable changes in DOS.

\subsection{Conclusions}

In this Chapter, we have studied all-optical ultrafast switching of high-quality $\mathrm{Si}$ inverse opal photonic crystals by free-carrier effects as well as linear optics. We find good agreement between the linear reflectivity of the crystals and calculated stop bands in the frequency range of the band gap. Surprisingly the width and 


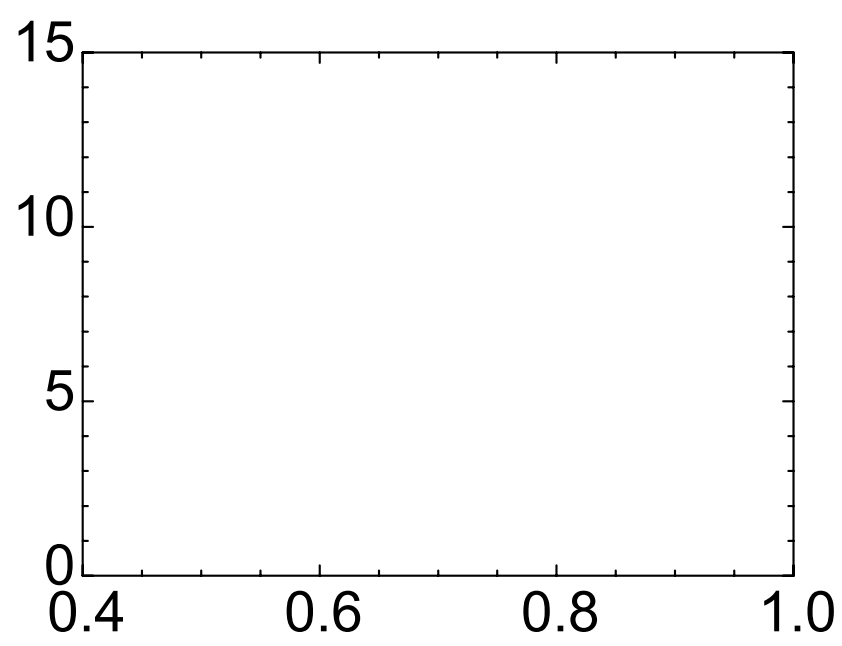

Figure 7.11: Frequency dependence of the density of states per volume in units of $\omega^{2} /\left(\pi^{2} c^{3}\right)$ normalized to $(\omega a / 2 \pi c)^{2}$ for an infinitely large inverse opal of close packed spheres, calculated at two different refractive indices of the semiconductor backbone $n_{S i}^{\prime}$. The black curve with closed symbols shows the DOS per unit volume for an unswitched Si crystal with $\epsilon_{S i}=12.75\left(n_{S i}^{\prime}=3.57\right)$. The red curve (open symbols) shows the DOS for an Si crystal in which the refractive index has been lowered to $n_{S i}^{\prime}=3.50$ $\left(\Delta n_{S i}^{\prime} / n_{S i}^{\prime}=-2.0 \%\right)$. All spectral features shift towards higher frequencies by $\Delta \omega / \omega=1.4 \%$ due to the reduced average refractive index.

frequency position of the first order peak is inconsistent with band structure calculations. Switching effects are studied as a function of the pump irradiance and the time delay between pump and probe pulses. Large ultrafast variations in reflectivity are observed in the range of second order Bragg diffraction. During the switching process, all spectral features in the observed stop bands, shift towards higher frequencies within a few hundred fs, indicating the absence of separate dielectric and air bands in our crystals. From a comparison to quasi-static with band structure calculations we infer a large refractive index change of about $2 \%$, while the absorption length in the crystal remains larger than the sample thickness. From subsequent density of state calculations, we surmise that the induced changes are large enough to strongly modify the density of states inside the crystal. We briefly discuss how the emission rate of internal light sources inside our switched crystals can be switched by switching the local radiative density of states in photonic crystals. 


\section{Recommendations}

\subsection{Applications}

We have studied photonic crystals made with semiconductor fabrication techniques near the telecom frequency range. It is therefore interesting to consider the applicability of on-chip ultrafast all-optical switching. Importantly, the pulse energy should be considerably reduced for two reasons: 1) applications will only arise if low-power pump lasers (diode lasers) can be used. 2) This is feasible for devices such as modulators wherein a cavity resonance with quality factor $Q$ is switched by one linewidth. Then, a small refractive index change $\Delta n^{\prime} / n^{\prime}=1 / \mathrm{Q}$ suffices. Assuming reported $\lambda^{3}$-sized cavities with $Q=10^{4}$ [139], $\Delta n^{\prime} / n^{\prime}$ is 100 times smaller than in our experiments. By pumping with a diffraction-limited pump pulse at above band gap frequencies $\left(\omega_{\text {pump }}=20000 \mathrm{~cm}^{-1}\right.$ for Si), free carriers are selectively excited inside the cavity volume only, requiring low pulse energies $\leq 50 \mathrm{fJ}$. Moreover, the observed decay times of less than 20 ps implies that switching could potentially be repeated at a rate $\geq 25 \mathrm{GHz}$. From heat diffusion theory, we estimate that the corresponding temperature increase in a Si sample is $\leq 10 \mathrm{~K}$. Therefore, we conclude that ultrafast photonic crystal switching also opens exciting opportunities in device applications, and we recommend switching experiments with reduced pump energy in high Q-cavities. 


\subsection{Adiabatic and non-adiabatic switching}

To study the transition between adiabatic and non-adiabatic switching timescales, we consider a cavity with resonance frequency $\omega_{1}$. The cavity is loaded by a probe pulse that is at resonance with the cavity. In case of a high $Q$ cavity, only part of the pulse will enter the cavity. A pump pulse is used to shift the resonance frequency of the filled cavity to frequency $\omega_{2}$ by either free-carrier or Kerr effects. The switching time-scale can be controlled by changing the duration of the pump pulse with a pulse stretcher. We identify two switching time-scale regimes: Firstly, if the switching takes place slowly, the eigenmode of the system will adjust to the new situation adiabatically and no transitions will be made. Consequently, the frequency of probe light will be changed from $\omega_{1} \rightarrow \omega_{2}$. Such a change could be observed if light that leaks out of the cavity is analyzed spectrally. Note that the frequency conversion will only take place if the cavity is switched while the probe light is inside the cavity. Secondly, it the non-adiabatic limit, at fast switching times, the adiabatic theorem no longer applies, and transitions from the cavity mode to other modes are allowed to take place.

In a planar multilayer cavity, for example, light in the cavity mode could make a transition to propagating modes parallel to the layers. Interesting prospects arise when cavities that are embedded in a three-dimensional band gap crystal are switched adiabatically. In such a situation, large frequency changes due to transitions to frequencies outside the band gap could take place.

We therefore recommend switching experiments in a planar multilayer cavities, in which the switching time of the cavity is varied by controlling the duration of the pump pulse by a pulse stretcher. Such an experiment allows to study the transition between the adiabatic and non-adiabatic switching regime.

Furthermore, we propose an experiment in which the coupling efficiency of probe light into a multilayer cavity is dynamically controlled by switching one of the cavity mirrors. Such an configuration can be considered the solid-state analogue of the experiment of Ref. [63], in which dynamic control over the coupling efficiency into a marcoscopic cavity allowed for adiabatic and reversible compression of a pulse into a single narrow cavity mode.

\subsection{Switched lifetime measurements with ultrafast shutters}

Switching photonic band gap crystals offers active control over the local density of states. An experimental demonstration of the switched emission rate of internal light sources due to a switched LDOS brings about several experimental challenges. 
First of all, a large effect on the emission lifetime will only be observed if the homogeneous line width of the sources is small compared to the magnitude of the induced frequency shift $\Delta \omega / \omega \approx 2 \%$. Suitable light sources may therefore be quantum dots, whose homogeneous line width can be less than $1.5 \times 10^{-4}[140,141]$. Secondly, the light sources should emit a sufficient amount of light during the period of the switching, which is typically tens of ps. The radiative lifetime of novel colloidal PbSe quantum dots that emit at IR wavelengths is of the order of 250 ns nanoseconds $[142,143]$ and thus about 4 orders of magnitude larger than the decay time of our free-carrier effect, thus to select photons that are emitted during the time of the switching only, we recommend the use of ultrafast optical Kerr gates [144], that allow to selectively measure the emission rate of light sources in the first $20 \mathrm{ps}$.

Large changes of the emission rate of light sources into a cavity mode can also be attained by switching a cavity in and out of resonance with an embedded emitter. We expect such changes to be measurable in planar cavities that include self assembled quantum dots. We propose an experiment in which the coupling between emitter and cavity strength in such systems is tuned at ultrafast timescales, resulting in variations in the emission rate.

\subsection{Non-monotonic effects due to Kerr and free carriers}

A first analysis of the reflectivity changes at zero time delay in the woodpile samples in Chapter 6 showed that the observed reflectivity decrease is consistent with a $\chi^{(3)}$ nonlinearity. Part of the effect, however, may be due to absorption, which is related to non-degenerate two-photon absorption of probe light in the presence of the pump field [71]. This assumption could be tested by varying the probe pulse energy, since this effect should also be proportional to the probe irradiance.

The effect at zero time delay may also be influenced by free carriers that are generated instantaneously. We therefore propose to lower the pump frequency to a value below the two-photon absorption edge of the Si. This configuration would allow a measurement of the $\chi^{(3)}$ nonlinearity without free-carrier effects. Recent experiments, in which the pump frequency was tuned across the two-photon absorption edge, have indeed demonstrated a transition between the free-carrier and $\chi^{(3)}$ effects. The $\chi^{(3)}$ effect at short time delays appears to be a combination of a positive Kerr changes and non-degenerate two-photon absorption [145]. 


\subsection{Z-scan experiments on poly silicon samples}

An important difficulty in the interpretation of switching measurements of poly crystalline samples such as the Si woodpiles in Chapter 6 and the Si inverse opals described in Chapter 7 was the unknown nonlinear absorption coefficient and Drude damping time of the backbone material. The values of $\beta$ and $\tau_{D}$ that we have deduced by comparing our data to theory strongly deviate from those found in crystalline $\mathrm{Si}$. In comparison, the GaAs absorption coefficient that we have deduced from our experiments on (crystalline) GaAs/AlAs structures is in much better agreement with its value in bulk crystalline GaAs. In addition, the effective optical mass of the carriers may also depend on the crystallinity of the material.

We conclude that a better interpretation of our results requires an independent measurement of $\beta$ of poly crystalline Si. We propose two ways in which this could be done: firstly, a z-scan experiment could be done on a non-photonic reference sample. A z-scan experiment (see Appendix C), also allows for the measurement of the nonlinear refraction in poly-Si, and could help to understand the nature of the observed $\chi^{(3)}$ effect. A difficulty is that a suitable reference sample must be grown under the exact same growth conditions as the sample itself. A second, more direct way of deducing the amount of absorption in the sample is to monitor the transmission of pump light during the experiment. From the amount of absorbed pump-light, the density of excited carriers can be estimated.

\subsection{Samples fabricated by etching techniques}

In the interpretation of our data on Si infiltrated opals, a problem arises, as the crystal structure away from the surface as well as the Si filling fraction cannot be determined accurately. Much better control over both the structure and the filling fraction can be obtained in samples that are made by etching of single crystalline materials, whose nonlinear optical properties are known from z-scan measurements (see Appendix C). Three-dimensional photonic crystals can then be fabricated by etching away material from a single crystalline wafer by combining electro-chemical etching with focussed ion beam milling [52], or by a two-step focused ion beam milling experiment [53]. An example of a GaP photonic crystal made by focussed ion beam milling is shown in Fig. 1.3D. We propose that such structures could be switched by either a three-photon process, or by utilizing the nonlinear refraction of GaP (see Appendix C). 
Appendix

\section{Drude description of excited semiconductors}

\section{A.1 Introduction}

To change the refractive index contrast in our photonic crystals, the solid semiconductor backbone with bulk dielectric constant $\epsilon_{B G}$ is excited with an optical pulse to generate a free-carrier plasma through two-photon absorption. The complex index of refraction of Si and GaAs is well described by the Drude model for moderately high densities of excited free carriers [73, 136, 146, 147].

\section{A.2 Basic assumptions}

The Drude model is a theory that was originally posted by Paul Drude in 1900 to describe metals $[3,94]$. It provides a practical way to estimate optical properties of a free-carrier plasma in metals or in semiconductors. There are four basic assumptions in the Drude model: First of all, it is assumed that an electron experiences a collision with a probability per unit time $1 / \tau_{D}$, where $\tau_{D}$ is known as the momentum relaxation time, or the mean free time of the electrons. The value of $\tau_{D}$ is assumed to be independent of the position and speed of the electron. Secondly, electron collisions are assumed to alter the velocity of the electrons instantaneously. Thirdly, the model neglects electron-electron interactions (independent electron approximation) and electron-ion interactions (free electron approximation) between 
collisions. Finally, the electrons are assumed to achieve thermal equilibrium with their surroundings only through collisions. After a collision, the velocity of the emerging electron is randomly directed, and its speed is related to the temperature of the region where the collision took place.

\section{A.3 Drude model for free carriers in Si}

Under the assumptions in Section A.2, the dielectric response of semiconductors with excited free carriers is well described by the Drude model, modified to include separate electron and hole contributions. Here we focus on $\mathrm{Si}$, and follow the notation of Ref. [136]. The real part of the dielectric constant is equal to:

$$
\epsilon^{\prime}(\omega)=\epsilon_{B G}^{\prime}(\omega)-\frac{N_{e h} e^{2}}{\epsilon_{0}}\left(\frac{1}{m_{e}^{\prime}}\left(\frac{\tau_{e}^{2}}{1+\omega^{2} \tau_{e}^{2}}\right)+\frac{1}{m_{h}^{\prime}}\left(\frac{\tau_{h}^{2}}{1+\omega^{2} \tau_{h}^{2}}\right)\right)+4 \pi \chi_{V B},
$$

where $\epsilon_{B G}^{\prime}$ is the bulk Si dielectric constant, $m_{e}^{\prime}=0.27$ and $m_{h}^{\prime}=0.38$ (in units of the electron mass $m_{0}$ ) are the electron and hole optical effective masses for $\mathrm{Si}$ [103]. $N_{e h}$ is the electron density, which is on the order of $10^{19} \mathrm{~cm}^{-3}$ in our experiments. $\tau_{e}$ and $\tau_{h}$ are the electron and hole momentum relaxation time, respectively. The first term between the brackets describes the contribution of the free electrons, and the second term the contribution of the holes. The final term is the susceptibility associated with intervalence-band absorption [148-150].

The imaginary part of the dielectric constant is equal to:

$$
\epsilon^{\prime \prime}(\omega)=\epsilon_{B G}^{\prime \prime}(\omega)-\frac{N_{e h} e^{2}}{\omega \epsilon_{0}}\left(\frac{1}{m_{e}^{\prime}}\left(\frac{\tau_{e}^{2}}{1+\omega^{2} \tau_{e}^{2}}\right)+\frac{1}{m_{h}^{\prime}}\left(\frac{\tau_{h}^{2}}{1+\omega^{2} \tau_{h}^{2}}\right)\right)+\frac{\lambda \sqrt{\epsilon_{B G}} \sigma_{V B}\left(\omega, N_{e h}\right) N_{e h}}{\pi} .
$$

where $\epsilon_{B G}^{\prime \prime}$ is the imaginary part of the unswitched background dielectric constant. The first term between the brackets describes the contribution of the free electrons, and the second term the contribution of the holes. Since the Drude model is essentially based on classical ideas, it only incorporates intraband absorption through the imaginary part of the dielectric constant with the refractive index obtained from Kramers-Kronig. Therefore, we include the final term in Eq. A.2, which accounts for the intervalence-band absorption cross Section $\sigma_{V B}\left(\omega, N_{e h}\right)$ of the excited carriers [148-150].

At higher carrier densities, the independent electron approximation breaks down. We correct for this by using an adjusted expression for the momentum relaxation rate of the electrons [118]: 


$$
\frac{1}{\tau_{e, h}}=\left(\frac{1}{\tau_{e, h}}\right)_{e, h-p n}+\gamma N_{e h}^{1 / 3},
$$

where $\left(\tau_{e}\right)_{e-p n}$ relates to electron-phonon interactions. The second term takes into account electron-electron and electron-hole scattering, which depends on the average distance between the carriers, and thus on $N_{e h}^{1 / 3}$. In single crystalline $\mathrm{Si}$, in the range of our experiment, the resulting values for $\tau_{e}$ and $\tau_{h}$ are of the order of $10^{-13}$ $\mathrm{s}$ [73].

We combine the effects of the electrons and the holes by using an optical effective mass $m_{\text {opt }}^{*}=\left(m_{e}^{\prime-1}+m_{h}^{\prime-1}\right)^{-1}=0.15 m_{0}$. We assume that the absorption in the unswitched crystal is zero $\left(\epsilon_{B G}^{\prime \prime}=0\right.$, which is valid in our experiments, since the probe frequencies are chosen well below the electronic band gap of the semiconductors. We assume that $\omega \tau_{e, h} \ll 1$. This assumption is validated since in our experiments in poly crystalline $\mathrm{Si}, \omega \tau_{e, h} \approx 12$, at $\omega=6660 \mathrm{~cm}^{-1}$, where we have used a relaxation time $\tau_{D}=10^{-14}$ that deduced for poly crystalline silicon by Ref. [91]. Under the assumptions above, the dielectric constant is approximated by:

$$
\epsilon\left(\omega, N_{e h}\right) \approx \epsilon_{B G}-\left(\frac{\omega_{p}}{\omega}\right)^{2}\left(1-\frac{i}{\omega \tau}\right)+\frac{i \lambda \sqrt{\epsilon_{B G}} \sigma_{I B}\left(\omega, N_{e h}\right) N_{e h}}{\pi},
$$

where the plasma frequency is defined as $\omega_{p} \equiv N_{e h} e^{2} / \epsilon_{0} m_{o p t}^{*}$. The interband absorption cross section in $\mathrm{Si}$ is about $10^{-17} \mathrm{~cm}^{2}$ at frequency $\omega=5000 \mathrm{~cm}^{-1}$ and $N_{\text {eh }}=$ $2 \times 10^{19} \mathrm{~cm}^{-3}[68,149]$. The resulting absorption length in $\mathrm{Si}$ is of the order of tens of microns, and intervalence-band absorption in Si will therefore neglected in our analysis. Consequently, the expression for $n(\omega)$ reduces to

$$
n(\omega)=\sqrt{\epsilon(\omega)} \simeq \sqrt{\epsilon_{B G}(\omega)-\left(\frac{\omega_{p}}{\omega}\right)^{2}\left(1-\frac{i}{\omega \tau_{D}}\right)} .
$$

Here, the approximation $x \ll a, \sqrt{a-x^{2}}=\sqrt{a}-x^{2} /(2 \sqrt{a})+\ldots$ is used. Thus, for small changes $\left(\omega_{p} / \omega\right)^{2} \ll \epsilon_{B G}$, the refractive index is equal to

$$
n(\omega) \simeq \sqrt{\epsilon_{B G}}-\left(\frac{\omega_{p}}{\omega}\right)^{2} \frac{1}{2 \sqrt{\epsilon_{B G}}\left(1-\frac{i}{\omega \tau_{D}}\right)}=n_{B G}(\omega)-\left(\frac{\omega_{p}}{\omega}\right)^{2} \frac{1}{2 n_{B G}\left(1-\frac{i}{\omega \tau_{D}}\right)},
$$

which can be separated into a real and an imaginary $n^{\prime}$ and $n^{\prime \prime}$. After insertion of the expression for $\omega_{p}$, we obtain for the real part of the refractive index

$$
n^{\prime}(\omega) \simeq n_{B G}-\left(\frac{\omega_{p}}{\omega}\right)^{2} \frac{1}{2 n_{B G}}=n_{B G}-\frac{e^{2} N_{e h}}{2 n_{B G} \epsilon_{0} m_{e} m_{o p t}^{*} \omega^{2}} .
$$


This expression clearly demonstrates that the refractive index change is proportional to $N_{e h}$, and inversely proportional to the optical effective mass $m^{*}$. In $\mathrm{Si}$, the approximation in Eq. A.7 is valid to within $0.2 \%$ for carrier densities up to $10^{20}$ $\mathrm{cm}^{-3}$. For the imaginary part we find

$$
n^{\prime \prime}(\omega) \simeq\left(\frac{\omega_{p}}{\omega}\right)^{2} \frac{1}{2 n_{B G} \omega \tau_{D}}=\frac{e^{2} N_{e h}}{2 n_{B G} \epsilon_{0} m_{e} m_{o p t}^{*} \omega^{3} \tau_{D}} .
$$

The corresponding absorption length of probe light $\ell_{a n s}$ is obtained via $\ell_{a b s}=$ $1 /\left(2 k_{0} n^{\prime \prime}\right)$, where $k_{0}=\omega / c$ :

$$
\begin{aligned}
\ell_{a b s} & =\left[2 \frac{\omega}{c}\left(\frac{\omega_{p}}{\omega}\right)^{2} \frac{1}{2 n_{B G} \omega \tau_{D}}\right]^{-1}=\left[\left(\frac{\omega_{p}}{\omega}\right)^{2} \frac{1}{\tau_{D} n_{B G} c}\right]^{-1} \\
\rightarrow & \ell_{a b s}=\left(\frac{\omega}{\omega_{p}}\right)^{2} \tau_{D} n_{B G} c=\frac{\omega^{2} \epsilon_{0} m_{e} m_{o p t}^{*} \tau_{D} n_{B G} c}{e^{2} N_{e h}} .
\end{aligned}
$$

Thus for carrier densities of the order of $10^{20} \mathrm{~cm}^{-3}$, the absorption length in excited $\mathrm{Si}$ is inversely proportional to the carrier density $N_{e h}$. Furthermore, the amount of absorption depends on the material specific value $\tau_{D}$, and on the optical effective mass of the carriers $m^{*}$.

\section{A.4 Drude description of excited carriers in GaAs}

Due to the different electronic band structure of GaAs compared to $\mathrm{Si}$, the properties of excited free carriers also differ from those in Si. The first important difference is the 2.6 times reduced optical effective mass $m_{\text {opt }}^{*}$ compared to Si, which implies that the same carrier density will induce a 2.6 larger change in the refractive index (see Eq. A.7). Table A.1 shows the room temperature values for the electron- an hole optical effective masses that were taken from literature. A second distinction of GaAs is that at the carrier densities in our experiments, electron-electron interactions dominate the momentum scattering. The value of $\gamma$ in Eq. A.3 is about $4 \times 10^{5} \mathrm{~m}^{-1}$ [118]. At the typical carrier densities of about $10^{19} \mathrm{~cm}^{-3}$ in our experiment this corresponds to a short relaxation time $\tau_{e} \approx 20 \mathrm{fs}$. A third important difference is related to the band structure of GaAs. In excited GaAs for wavelengths below 3 microns the probe absorption is dominated by a strong interband absorption of excited carriers in the $\Gamma$-valley, which can be further excited into the $X$-valley [110]. Free carrier absorption is negligible below $\lambda=2 \mu \mathrm{m}$ [119]. The resulting absorption 


\begin{tabular}{l||l|l|} 
& crystalline Si $(\mathrm{T}=300 \mathrm{~K})$ & crystalline GaAs $(\mathrm{T}=300 \mathrm{~K})$ \\
\hline$m_{e}$ & $0.27 m_{0}$ & $0.065 m_{0}$ \\
$m_{h}$ & $0.38 m_{0}$ & $0.51 m_{0}$ \\
$m_{0}$ & $9.12 \times 10^{-31} \mathrm{~kg}$ & $9.12 \times 10^{-31} \mathrm{~kg}$ \\
$m_{\text {opt }}^{*}$ & $0.15 m_{0}$ & $0.0577 m_{0}$ \\
$\tau_{e}$ & - & $9.25 \times 10^{-14} \mathrm{~s}$ \\
$\tau_{h}$ & - & $2.18 \times 10^{-14} \mathrm{~s}$ \\
$\tau_{D}$ & $10^{-13} \mathrm{~s}^{*}$ & - \\
$\sigma_{\text {inter }}$ & - & $5.6 \times 10^{-18} \mathrm{~cm}^{2}[119]$
\end{tabular}

Figure A.1: Tabulated values for the electron- and hole optical effective masses, at room temperature. Values for Si taken from Refs. [73, 103]. GaAs data taken from Ref. [102]. * In polycrystalline Si, a reduced value $\tau_{D}=10^{-14}$ was deduced by Ref. [91]

coefficient can be discussed in terms of an (almost frequency independent) crosssection $\alpha_{\text {inter }}=\sigma_{\text {inter }} N_{\text {eh }}$ [119]. At $\lambda=1.5 \mu \mathrm{m}$, we deduce a value $\sigma_{\text {inter }}=5.6 \pm 0.5 \times$ $10^{-18} \mathrm{~cm}^{2}$ from the data of Ref. [119]. The total absorption coefficient is equal to:

$$
\alpha_{\text {total }}=\alpha_{\text {intra }}+\alpha_{\text {inter }}
$$

where $\alpha_{\text {intra }}$ is the absorption coefficient related to the Drude model.

In summary, the Drude model is expected to yield a good prediction of the complex refractive index change in excited GaAs of carrier densities up to about $10^{19} \mathrm{~cm}^{-3}$, where free-carrier absorption is negligibly small. At higher densities, however, the complex refractive index change is no longer correctly described by the Drude model, since absorption in GaAs is dominated by additional absorption processes. Using the Drude model to describe GaAs at high carrier densities therefore yields unphysically low values of the momentum relaxation time $\tau_{D}$. Additional absorption processes such as interband absorption and electron-electron scattering must be taken into account separately to correctly predict the complex refractive index at carrier densities above $10^{19} \mathrm{~cm}^{-3}$ in GaAs. We note that these absorption processes will also modify the real part of the refractive index due to the Kramers-Kronig relations, and the corresponding susceptibility $\chi$ should be therefore included in A.1. 



\section{Appendix}

\section{WPC model}

This Appendix will discuss the basic assumptions and the working procedure of the WPC ('WoodPile Crystal') model that was introduced in Section 6.4. We will test the numerical convergence by comparing to exact results. We will go through the program without going into much mathematical detail. A complete mathematical description of the model can be found in Ref. [125].

\section{B.1 Solving Maxwell's equation for a photonic crystal by Fourier expansion}

The behavior of electromagnetic waves is described by Maxwell's equations. In the absence of sources and currents, as is normally the case in an externally illuminated dielectric photonic crystal, Maxwell's equations can be combined into a single second-order differential equation for the electric field $\mathbf{E}(\omega, \mathbf{r})$, the Helmholtz equation:

$$
\frac{\omega^{2}}{c^{2}} \mathbf{E}(\mathbf{r})-\epsilon(\mathbf{r})^{-1} \nabla \times \nabla \times \mathbf{E}(\mathbf{r})=0,
$$

where $\epsilon_{0}$ is the free space permittivity, c the speed of light in vacuum, and a harmonic time dependence with frequency $\omega$ is assumed. In our crystals, the dielectric constant $\epsilon(\mathbf{r})$ is a periodic function of $\mathbf{r}$. Consequently, the most common approach to solving equation B.1 periodic media is fully expanding $\epsilon(\mathbf{r})^{-1}$ in its Fourier components. For a 3D woodpile crystal the expansion has to be made in the $x, y$ and $z$ directions, where $x$ and $y$ are the directions in which the grating 
planes are periodic and $z$ the direction in which the gratings are stacked. The field in the crystal is expanded in the same components and the equation can be solved.

A full Fourier expansion has some drawbacks, however. First of all, a Fourier expansion implies a perfect and infinite crystal. While this approach is necessary to obtain the photonic band structure of a crystal it cannot be used to obtain the reflectivity of a finite sample since the boundaries of the crystal are not defined. Secondly, a full expansion demands long calculation times, and uses large amounts of internal computer memory. Therefore, the expansion needs to be truncated, leading to either considerable truncation errors or long calculation times. Finally, the permittivity $\epsilon(\mathbf{r})$ in this type of calculations must be real and constant, hence dispersion and absorption can not be taken into account.

\section{B.2 The Exact Model Method (EMM)}

The WPC algorithm treats the woodpile crystal as a stack of gratings which extends infinitely in the lateral $x$ and $y$ direction, and is finite in the $z$ direction. The Helmholtz equation in the crystal can now be solved by the so-called exact modal method (EMM). The EMM is an extension of grating calculations by Refs. [151,152]. For each layer, the equation is solved separately and the solutions for the individual layers are connected via boundary conditions.

The EMM algorithm solves equation B.1 for each layer under the assumption that within each layer, the permittivity is a function of the $x$ or $y$ coordinate only. Equation B.1 is now decoupled into two first order differential equations:

$$
\mathbf{E}=\left(\omega \epsilon_{0} \epsilon(\mathbf{r})\right)^{-1} \nabla \times \mathbf{H}, \quad \mathbf{H}=\left(\omega \mu_{0}\right)^{-1} \nabla \times \mathbf{E} .
$$

We make the simplifications: Since the permittivity within each layer depends on a single coordinate, $\mathbf{E}$ and $\mathbf{H}$ can be written in terms of either the $x$ - or the $y$-component only. Without loss of generality, we assume the periodicity to be in the $x$ direction, and we eliminate the $y$ component. Writing out equations B.2 componentwise then results in a so-called evolution equation:

$$
\delta_{z}^{2}\left(\begin{array}{c}
E_{x} \\
H_{x}
\end{array}\right)=\mathbf{L}\left(\begin{array}{c}
E_{x} \\
H_{x}
\end{array}\right)=\left(\begin{array}{cc}
L_{\mu_{x}} & 0 \\
0 & L_{\epsilon_{x}}
\end{array}\right)\left(\begin{array}{c}
E_{x} \\
H_{x}
\end{array}\right)
$$

where $\delta_{z}$ is the differential operator in the $z$ direction, and $L_{\mu_{x}}$ and $L_{\epsilon_{x}}$ are complex functions of $\epsilon(x), \mu(x), \omega$ and the differential operators $\delta_{x}$ and $\delta_{y}$. The evolution operator $\mathbf{L}$ describes the evolution of $E_{x}$ and $H_{x}$ with increasing $z$. The calculation needs to be done for the $x$-direction only, the other components of the fields can be 
found using equations B.2. Subsequently, incoming fields on the layer can be now expressed in eigenfunctions of the evolution operator $\mathbf{L}$. The mathematical details can be found in Ref. [125]. From the operator L, matrices are obtained that connect $\mathbf{E}_{x}$ and $\mathbf{H}_{x}$, expressed in eigenfunctions, at both sides of the layer.

Once the solution for a layer has been found, it is coupled to the solutions for the adjacent layers via the continuous boundary condition condition for the tangential components of $\mathbf{E}$ and $\mathbf{H}$. By multiplication with a transformation matrix, the fields at the boundary of a layer $j$ with periodicity in the $x$ direction are transformed to the coordinate system of the next layer $(j+1)$, where the periodicity can be in the other $(y)$ direction. This process is repeated for each layer in the crystal, and finally, we can couple the equations for the internal and the external fields, and thus obtain a reflectivity spectrum.

Up to here, the result of the EMM algorithm is exact. However, expressing the incoming field for a layer in eigenfunctions of the evolution operator for that layer can only be done exactly if an infinite number eigenfunctions are taken into account. For numerical implementation, the series has to be truncated. In Ref. [125] it is proved that truncation is possible and that the difference with the exact result decreases exponentially with an increasing number of terms in the expansion. The convergence of the program is investigated in Section B.6.

\section{B.3 Advantages and limitations of the EMM algorithm}

The EMM algorithm has two important calculational advantages with respect tot other methods that solve the Helmholtz equation for a photonic crystal. First of all, the algorithm makes uses of the symmetry of the structure by decomposing each layer in one dimension only. The algorithm therefore converges faster than methods that use a Fourier decomposition in all dimensions. The number of functions $N$ to be solved scales with $M^{2}$, where $M$ is the number of functions used per coordinate. In standard Fourier decomposition, the scaling behavior is $N=M^{3}$. Secondly, the algorithm can handle complex or frequency-dependent refractive indexes, which is essential in the descriptions of switched woodpile photonic crystals, whose refractive index is both complex and highly dispersive. An important limitation of the EMM algorithm is that it preferentially handles structures consisting of infinite rectangular rods, as it is especially designed for calculations on woodpile crystals and stacks of gratings. Consequently, is is a great challenge to use the algorithm on structures other than stacked gratings, like opals and inverse opal photonic crystals [127]. 


\section{B.4 Test on 1D Bragg stack with comparison to exact results}

To verify if the WPC program works properly, we have calculated the reflectivity spectrum of a 1D Bragg stack and compared it to the analytic result obtained by transfer matrix calculations [153]. The Bragg stack consisted of a 11 layers with alternating refractive index index 1 and 3. The layer thickness of $200 \mathrm{~nm}$ was chosen such that the center frequency of the stop band of the Bragg stack (6000 $\mathrm{cm}^{-1}$ ) is comparable to that of the stop band our woodpile crystals. To model the Bragg stack in the WPC program, the width of the rods was increased until they touched each other. $M=9$ waves were used in the expansion for each layer to ensure sufficient convergence (see Section B.6). Reflectivity spectra for both calculation are shown in Fig. B.1. Excellent agreement within $\Delta R=0.2 \%$ is found over the whole frequency range (see Fig. B.1, lower panel). The largest residual differences are observed near steep slopes in the spectrum.

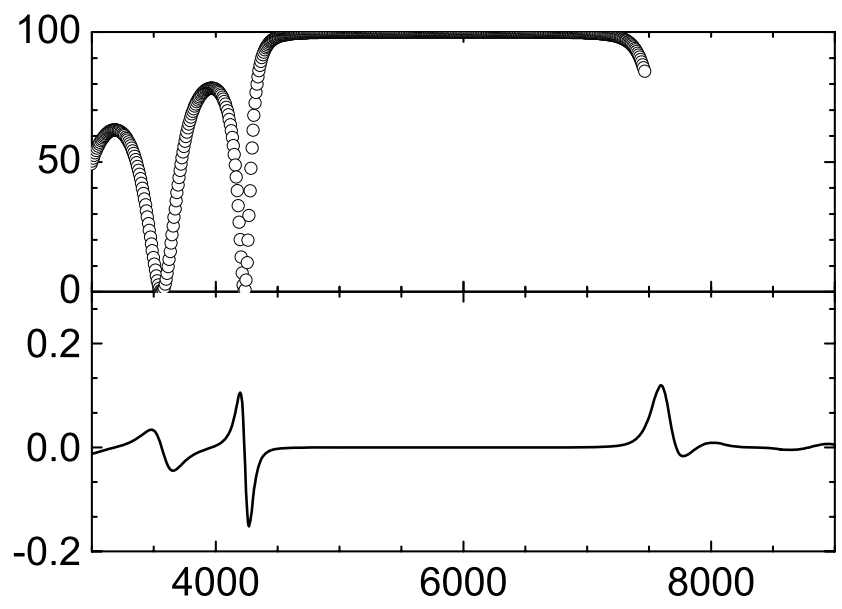

Figure B.1: Comparison between reflectivity calculated by the WPC program and exact result for a eleven-layer Bragg stack. Agreement within $0.2 \%$ is found between both results.

\section{B.5 Calculation time}

The accuracy of the WPC calculations is governed by the number of plane waves $M$, which can be specified by the user. By increasing $M$ to reduce the truncation error, however, at some point the calculation will take an unpractical amount of 
time. To find the maximum numbers $M$ which can still be used in practice, a set of calculations with increasing number of plane waves was done for a perfect woodpile crystal of five layers thickness, so no structural imperfections were taken into account. Parameters are: rod width $a=180 \mathrm{~nm}$, spacing between rods $b_{1}=$ $b_{2}=650 \mathrm{~nm}$, layer heights $c$ of $200 \mathrm{~nm}$, refractive index of the rods 3.45 (values as in Fig. 5 in Ref. [125]). The reflectivity was calculated for a single frequency in the stop gap. The corresponding calculation time is plotted versus the number of plane waves in Fig. B.2. The calculation time increases exponentially with $M$ : for every 2 plane waves that are added, the calculation time increases by a factor of 3 . In our calculations we have used $M=7$ waves, which limits the calculation time per frequency point to less than 15 seconds, allowing high resolution reflectivity spectra to be calculated in about 1 hour.

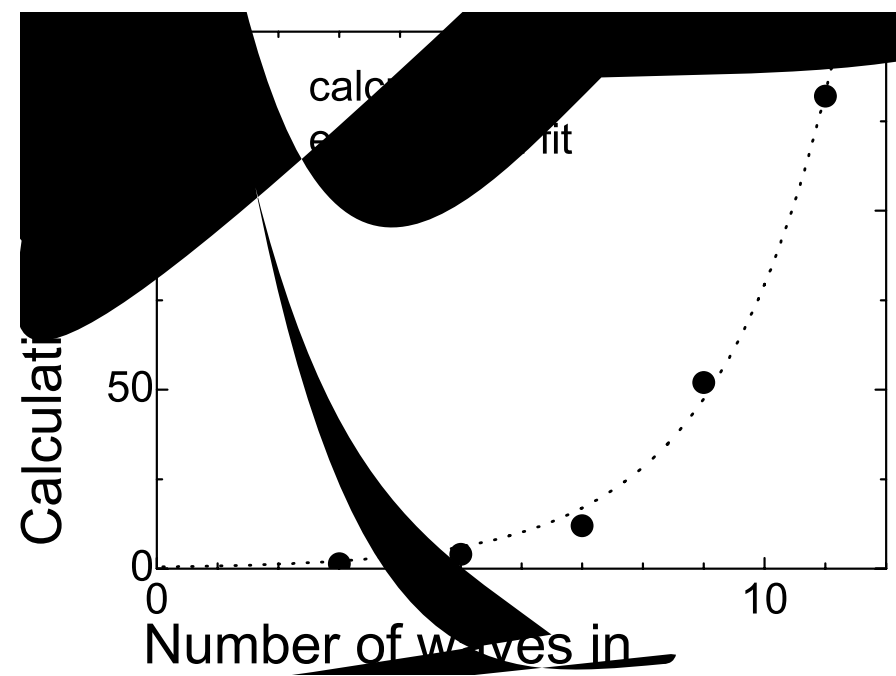

Figure B.2: Calculation time for the reflectivity at one fixed frequency in the stop band of a perfect woodpile crystal, as a function of the number of plane waves $M$ used in the calculation. The calculation time increases exponentially with $M$.

\section{B.6 Convergence test for a 3D woodpile crystal}

To investigate if the chosen number of plane waves in the expansion $M=7$ provides sufficiently accurate spectra, the reflectivity at perpendicular incidence of the perfect woodpile crystal was calculated for 300 frequency points between 0 and 
$10000 \mathrm{~cm}^{-1}$ and several values of $M$. The calculation was repeated for $M=5,7$ or 9 waves in the expansion. The resulting spectra are plotted in the upper panel of Fig. B.3. Up to $\omega=7000 \mathrm{~cm}^{-1}$, the three spectra overlap within $\Delta \mathrm{R}=0.3 \%$ indicating that in this range, the method already converges for $M=5$. The lower panel in Fig. B. 3 shows the difference between the calculation for $M=5$ and $M=7$ with the calculated spectrum for $M=9$. The reflectivity difference between $M=7$ and $M=9$ is less than $\Delta R=0.1 \%$ for frequencies below $7500 \mathrm{~cm}^{-1}$, showing excellent convergence in this range. At frequencies in the range $7500-1000 \mathrm{~cm}^{-1}$, large differences of up to $\Delta \mathrm{R}=30 \%$ appear for $M=5$. As the $M$ is increased to 7 , the maximum deviation from the $M=9$ spectrum is reduced to $\Delta \mathrm{R}=7 \%$, indicating that the spectrum starts to converge.

Despite the moderate convergence of the spectra at frequencies above 7500 $\mathrm{cm}^{-1}$, we decided to use $M=7$ plane waves to avoid extreme calculation times. The calculation of the stop band for a perfect woodpile crystal with 7 waves takes about half an hour. However, when imperfections are introduced, the calculation time increases by a factor three to four due to the larger unit cell dimensions [125]. We conclude that the calculated WPC spectra in Chapter 6 will be less accurate at high frequencies above the stop band edge and are therefore disregarded in the comparison to the experiments.

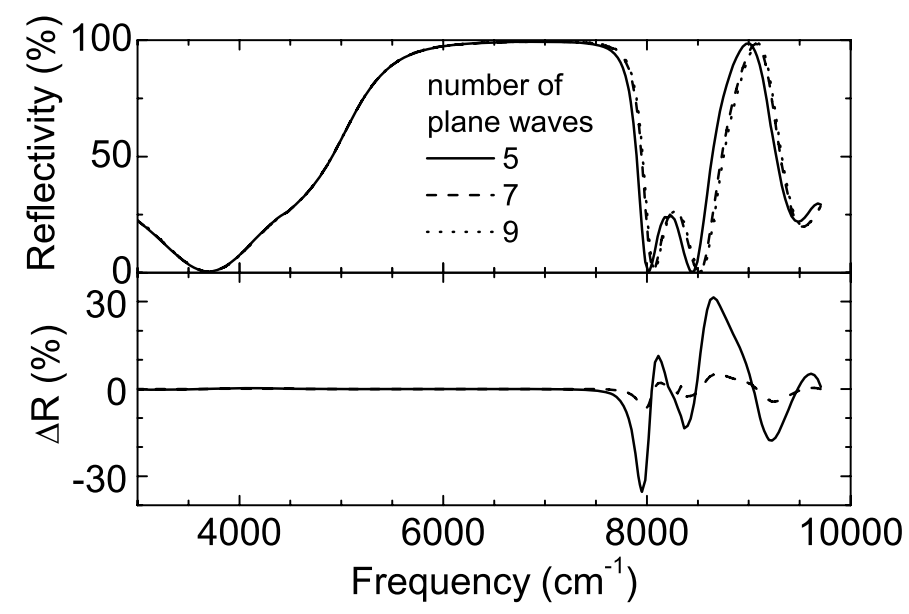

Figure B.3: (upper panel) Calculated stop band for a perfect five-layer woodpile crystal, using respectively 7 or 9 wave functions in the expansion of the field in eigenfunctions of the evolution operator. (lower panel) Excellent convergence within $\Delta R=0.1 \%$ is observed at frequencies below $7500 \mathrm{~cm}^{-1}$ for $M=7$, while at higher frequencies, errors of up to $\Delta R=7 \%$ are observed. 


\section{Appendix}

\section{Z-scan measurements}

\section{C.1 Introduction}

In our switching experiments, we use two-photon absorption to excite a free-carrier plasma in the semiconductor backbone of photonic crystals. It is therefore important to determine the two-photon absorption coefficient of these materials at the pump frequencies used in our experiments. Several techniques can be used to obtain then nonlinear refraction and absorption of materials. For example, high sensitivities have been demonstrated by spectrally resolved pump-probe measurements [154]. Unfortunately, this technique is sensitive to sample inhomogeneity and relies on a good spatial overlap of pump and probe beam.

An elegant alternative method is the z-scan technique that was first demonstrated by Sheik Bahae et al. to measure the nonlinear refraction $n_{2}$ of semiconductors $[155,156]$. A z-scan is a simple and robust measurement of the transmission of a single beam that is focussed by a lens. The focal length of the lens is chosen such that the focal depth is much larger than the sample thickness. Transmitted power of a focused laser beam is measured while the sample is scanned in the z-direction, along the optical axes of the beam, see Fig. C.1. The nonlinear refraction results in both Kerr-lensing due to the change in real part of the refractive index, as well as attenuation of the transmitted power due to nonlinear absorption. Both effects will attain a maximum if the sample is located in the beam waist $(z=0)$.

To separate the refractive and absorptive effects, two scans must be made. The first experiment is a closed aperture z-scan, in which refraction and absorption are measured simultaneously. A diaphragm that is placed in front of the detector 
blocks part of the linearly transmitted light. For example, a positive $n_{2}$ will induce a positive Kerr lens in the sample, which will guide more light into the detectors if the sample is placed at a positive z-position in between the beam waist and detector (see Fig. C.1). The transmitted intensity will be reduced if the sample is placed in between the lens and beam waist. The nonlinear refraction will cause an asymmetry in the z-scan data. The second experiment is a an open aperture $\mathrm{z}$-scan, in which the aperture is removed, and all transmitted light is collected. The effect of nonlinear refraction is thus removed. The resulting curve only depends on the induced absorption, and is therefore symmetric around the beam waist $(\mathrm{z}=0)$. By subtracting the open aperture data from the closed aperture data, the refractive and absorptive effects can be separated [97, 100, 114, 157].

In our switching experiments we are mostly interested in the nonlinear absorption coefficients near the two-photon absorption edge of Si and GaAs. Therefore this appendix only describes open aperture z-scan experiments that were done on $\mathrm{Si}, \mathrm{GaAs}$, and $\mathrm{GaP}$ single crystalline wafers. To the best of our knowledge the results presented here are the first measurements of the two-photon absorption coefficient of $\mathrm{Si}$ an GaAs near the two photon absorption edge $\left(\hbar \omega \approx \frac{1}{2} E_{g a p}\right)$. We are also unaware of any prior measurement of the three-photon absorption coefficient of $\mathrm{GaP}$ in the three-photon absorption frequency range $\left(\frac{1}{3} E_{\text {gap }}<\hbar \omega<\frac{1}{2} E_{\text {gap }}\right)$.

\section{C.1.1 Experimental}

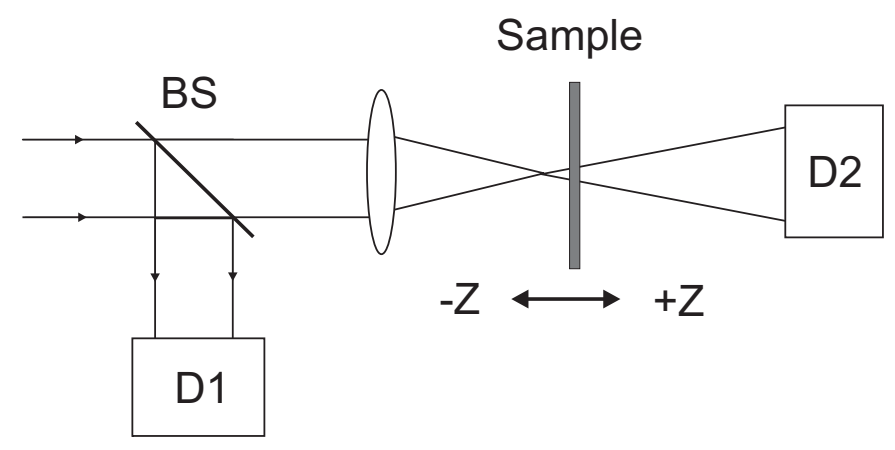

Figure C.1: Schematic drawing of the z-scan setup. Incoming laser beam from our OPA is split by a beam splitter (BS). Two InGaAs photodiodes are used to monitor the output variation of the OPA (D1) as well as the transmitted signal (D2). The detector signals are measured as a function of sample position $z$.

Fig. C.1 shows a schematic drawing of our z-scan setup. The power of the 
incoming beam is monitored by detector D1. The beam is focused by a lens with focal length $f$. The sample, typically a double-side polished wafer, is placed on a translation stage that scans the sample along the z-direction. The zero position is taken at the beam waist. The transmitted power is collected by a second lens (not shown) and measured by a InGaAs photodiode D2.

The shape of the transmission curve strongly depends on pump irradiance, and pump power stability is therefore essential for the correct interpretation of the experimental data. Each pulse was therefore measured individually using the detection scheme described in Chapter 4. Both pump-monitor detector signals (D1) and transmission signals (D2) for each pulse event are stored. We minimize the effect of pulse-to-pulse variations in the laser output (which can be as high as $10 \%$ ) by selectively removing all pulses with energy beyond a certain threshold. In all experiments, the number of pulses collected and the threshold were chosen such that the standard deviation in pump energy remained below $3 \%$. Typically between 2500 and 10000 pulses were collected for each datapoint. The corresponding standard error in the transmission was typically better than $\delta \mathrm{T} / \mathrm{T}<1 \%$, which is sufficiently sensitive for our z-scan measurements. The sensitivity can be further increased by narrowing the pulse energy range, at the price of longer integration times.

\section{C.1.2 Model}

To interpret the z-scan data, we have numerically calculated the nonlinear transmission of a Gaussian beam through a thin slab. The beam width of a diffraction limited gaussian beam is

$$
w(z)=\sqrt{w_{0}\left[1+\left(\frac{\lambda z}{\pi w_{0}}\right)^{2}\right]},
$$

where $\lambda$ is the wavelength, $\mathrm{z}$ is the sample position relative to the focus [106]. The diffraction limited width of the beam waist is equal to:

$$
w_{0}=\frac{f \lambda}{\pi w_{b}},
$$

where $f$ is the focal length of the lens and $w_{b}$ is the Gaussian width of the unfocused beam at the position of the lens. At each wavelength, $w_{b}$ was determined by a knife edge scan (see Fig. 4.2).

In our calculation we have discretized the sample into $256 \times 256$ independent transmission channels of $10 \times 10 \mu \mathrm{m}^{2}$. We have made sure that the lateral dimensions of each channel are smaller than the focus width $w_{0}$, to avoid discretization artifacts 
at $\mathrm{z}=0$. We use Eq. 3.4 to calculate the nonlinear transmission through each channel. Since our pump frequencies are in the two-photon absorption range $\frac{1}{2} E_{\text {gap }}<\hbar \omega<$ $E_{\text {gap }}$ where $\alpha=0$, we can simplify Eq. 3.4. The transmitted power through a sample with thickness $\mathrm{L}$, normalized to the linear transmission $(1-R)^{2}$ is equal to:

$$
T(z)=\frac{1}{1+I_{0}(z) \beta L},
$$

where $\mathrm{R}$ is the Fresnel reflectance at the front- and back-face of the sample, $\beta$ is the two-photon absorption coefficient, and $I_{0}(z)$ the irradiance at the sample interface after subtraction of the front-face reflection at position $\mathrm{z}$. The added calculated transmission of all channels is plotted versus sample position $z$. The adjustable parameter in this calculation is $\beta$.

\section{C.2 Results}

\section{C.2.1 Two-photon absorption in Si}

First we consider open aperture z-scan measurements on a double-side polished Si wafer with thickness $\mathrm{L}=360 \mu \mathrm{m}$. Normalized transmission is plotted in Fig. C.2 as a function of sample position z. Data were taken at two wavelengths: $\lambda=1630$ $\mathrm{nm}$ (open circles) and at $\lambda=1720 \mathrm{~nm}$ (closed squares). The measured data were normalized to the linear transmission away from the focus. We observe that in both scans, the transmission is strongly reduced as the sample scans through the focus. The curves are numerically calculated transmission data, where $\beta$ was used as fitting parameter. We find good agreement for $\beta=0.6 \pm 0.3 \mathrm{cmGW}^{-2}(\lambda=1630 \mathrm{~nm})$, and for $\beta=0.2 \pm 0.1 \mathrm{cmGW}^{-1}$ at $\lambda=1720 \mathrm{~nm}$. Fig. C.3 shows a z-scan of the same Si wafer at a pump wavelength $\lambda=2000 \mathrm{~nm}$, close to the two-photon absorption edge of $\mathrm{Si}$. The peak irradiance during this scan was $I_{0}=800 \pm 200 \mathrm{GWcm}^{-2}$. We find good agreement for $\beta=0.20 \pm 0.05 \mathrm{cmGW}^{-2}$.

\section{C.2.2 Two-photon absorption in GaAs}

Additionally, we have performed open aperture z-scan experiments on a doubleside polished GaAs wafer with a thickness of $189 \mu \mathrm{m}$. Figure C.4 shows z-scan data taken at $\lambda=1720 \mathrm{~nm}$, just above the two-photon absorption edge of GaAs. The measured data were normalized to the linear transmission away from the focus. The peak irradiance was $I_{0}=366 \pm 60 \mathrm{GWcm}^{-2}$ in Fig. C. 4 . The strongly attenuated transmission near the waist of the beam indicates a strong nonlinear 


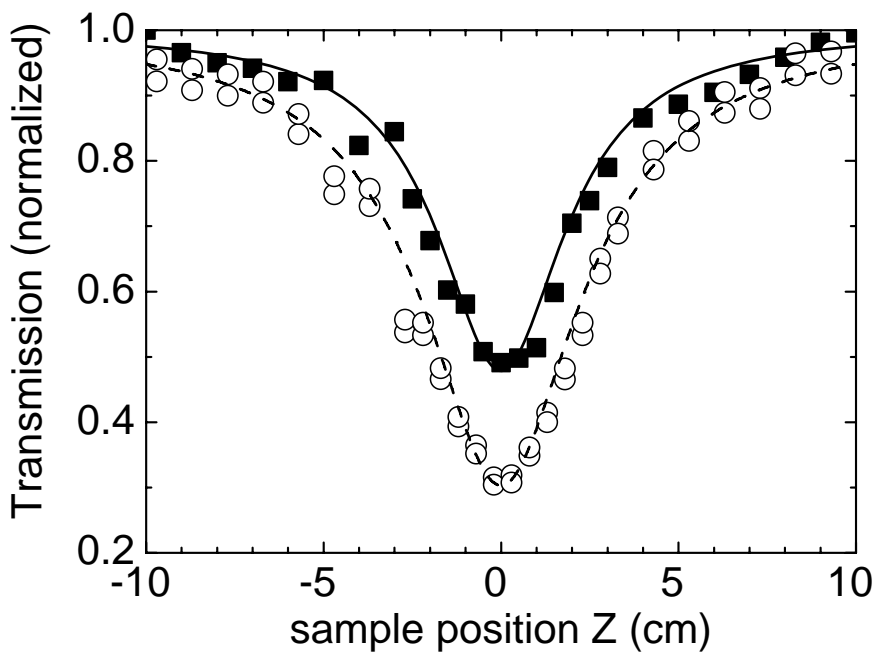

Figure C.2: Open aperture z-scan measurement for a $360 \mu \mathrm{m}$ thick double-side polished Si wafer. Open circles: $\lambda=1630 \mathrm{~nm}, f=100 \mathrm{~mm}, I_{0}=385 \pm 40 \mathrm{GWcm}^{-2}$. Closed squares: circles, $\lambda=1720$ $\mathrm{nm}, f=100 \mathrm{~mm}, I_{0}=315 \pm 40 \mathrm{GWcm}^{-2}$. The curves are calculated transmission using $\beta=0.6 \pm 0.3$ $\mathrm{cm} \mathrm{GW}^{-2}$ (dashed curve, $\lambda=1630 \mathrm{~nm}$ ) and $\beta=0.2 \pm 0.1 \mathrm{~cm} \mathrm{GW}^{-2}$ (solid curve, $\lambda=1720 \mathrm{~nm}$ )

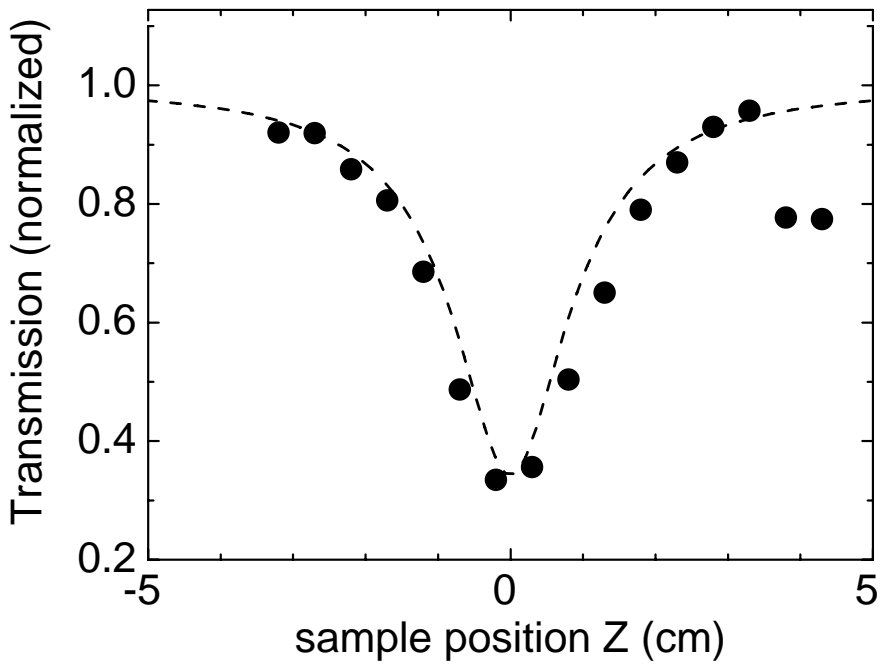

Figure C.3: Open aperture z-scan measurement for a $360 \mu \mathrm{m}$ thick double-side polished Si wafer. Pump parameters: $\lambda=2000 \mathrm{~nm}, f=150 \mathrm{~mm}, \tau_{p}=130 \mathrm{fs}_{\mathrm{s}} I_{0}=800 \pm 200 \mathrm{GWcm}^{-2}$. The dashed curve represent the calculated transmission using $\beta=0.20 \pm 0.05 \mathrm{cmGW}^{-2}$ 
absorption. The curve is calculated using $\beta$ as an adjustable parameter. We find good agreement for $\beta=1.5 \pm 0.5 \mathrm{cmGW}^{-2}$. Fig. C.5 shows z-scan data for GaAs at a shorter wavelength $\lambda=1630 \mathrm{~nm}$. Here, we find good agreement for $\beta=3.5 \pm 1.0$ $\mathrm{cmGW}^{-2}$. The value $\beta=1.5 \pm 0.5 \mathrm{cmGW}^{-2}$ at the two-photon absorption edge is an order of magnitude smaller than the value $\beta=15.1 \pm 2.3 \mathrm{cmGW}^{-1}$ at $\lambda=1.27$ $\mu \mathrm{m}$ reported by Ref. [97]. We conclude that in GaAs, the two-photon absorption coefficient strongly decreases as the pump wavelength approaches half the band gap energy, allowing more homogeneous switching (see Chapter 3 ).

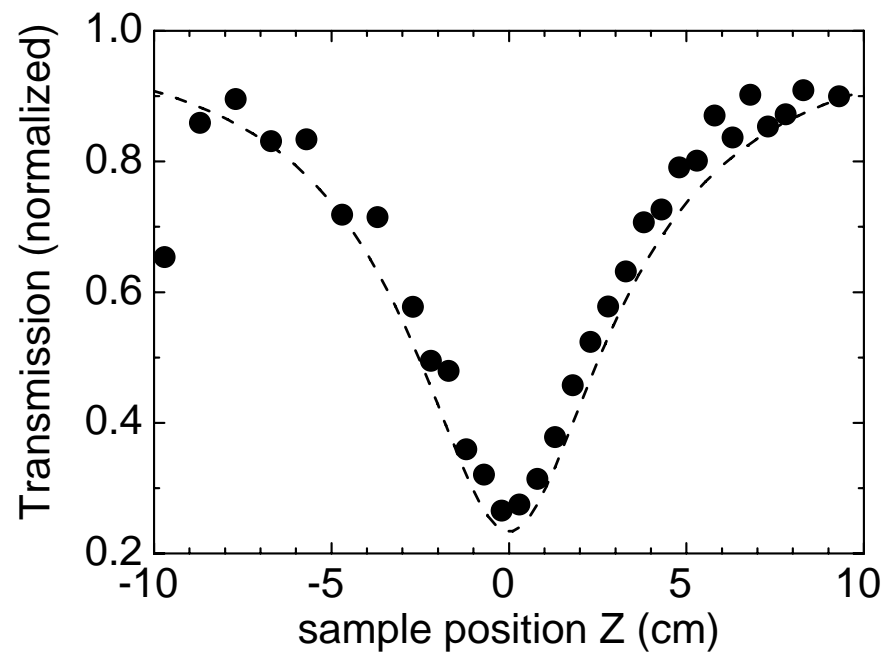

Figure C.4: Open aperture z-scan measurement for a $189 \mu \mathrm{m}$ thick double-side polished GaAs wafer. Pump parameters: $\lambda=1720 \mathrm{~nm}, f=100 \mathrm{~mm}, \tau_{p}=130 \mathrm{fs}_{,} I_{0}=366 \pm 60 \mathrm{GWcm}^{-2}$. The curve represents the calculated transmission using $\beta=1.5 \pm 0.5 \mathrm{cmGW}^{-2}$

\section{C.2.3 Three-photon absorption in gallium phosphide}

We have also obtained open aperture z-scan data for GaP. The pump wavelength was chosen in the range of three-photon absorption: $\frac{1}{3} E_{\text {gap }}<\hbar \omega<\frac{1}{2} E_{\text {gap }}$. We therefore neglect both linear and two-photon absorption in our analysis $(\alpha=\beta=0)$. The resulting equation for the nonlinear transmission of the sample, normalized to the linear transmission $(1-R)^{2}$ is:

$$
T(z)=\frac{1}{\sqrt{1+2 I_{0}(z)^{2} \gamma L}},
$$

where $\gamma$ is the three-photon absorption coefficient. 


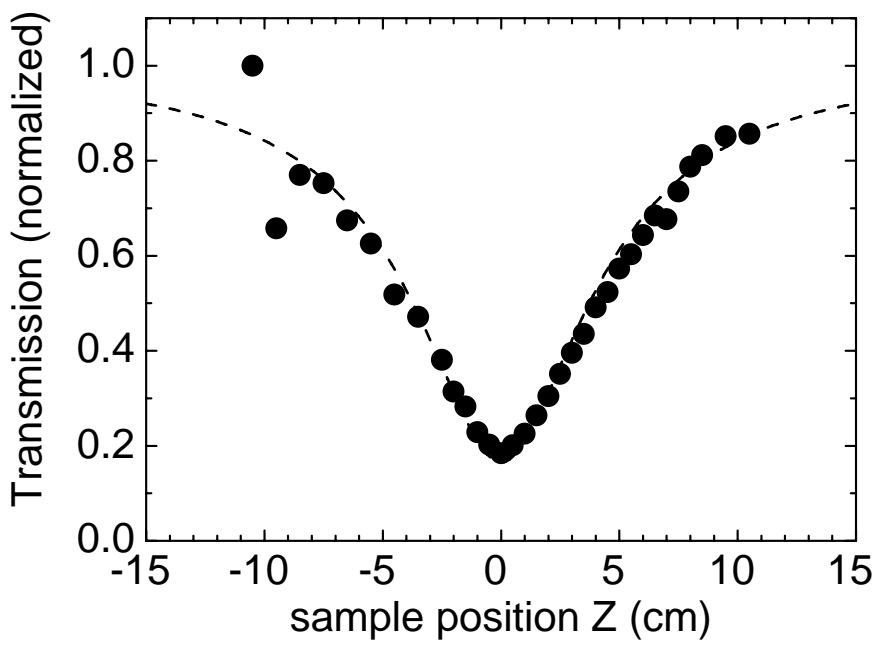

Figure C.5: z-scan measurement for a $189 \mu \mathrm{m}$ thick double-side polished GaAs wafer. Pump parameters: $\lambda=1630 \mathrm{~nm}, f=100 \mathrm{~mm}, \tau_{p}=130 \mathrm{fs}_{\mathrm{s}} \mathrm{I}_{0}=244 \pm 40 \mathrm{GWcm}^{-2}$. The curve represents the calculated transmission using $\beta=3.5 \pm 1.0 \mathrm{cmGW}^{-2}$

Fig. C.6 shows typical z-scan data taken at a wavelength $\lambda=1600 \mathrm{~nm}$, close to the three-photon absorption edge of GaP. We observe that the z-scan data in Fig. C.6 is asymmetric; the transmission of the sample at positive $\mathrm{z}$-values, where the sample is located in between the beam waist and the detector, is slightly elevated. This asymmetry indicates that nonlinear refraction also plays a role in this experiment. Consequently, the data are strictly speaking not an open aperture z-scan. From the shape of the curve we conclude that the sign of the nonlinear refraction in $\mathrm{GaP}$ is positive in the wavelength range $1400-1600 \mathrm{~nm}$. We therefore exclude that the asymmetry is caused by free-carrier generated by three photon absorption, since this would result in a negative refractive index change.

To obtain the three-photon coefficient $\gamma$ for $\mathrm{GaP}$, we disregard the relatively small nonlinear refraction. We compare our results to a calculated transmission curve, shown as a dashed curve in Fig. C.6. We have varied $\gamma$ until the depth of the minimum of the calculated scan matches the data. The calculated curve agrees reasonably with the data even though the refractive effects have not been taken into account. At $\lambda=1600 \mathrm{~nm}$, we deduce $\gamma=1.0 \pm 0.3 \times 10^{-3} \mathrm{~cm}^{3} \mathrm{GW}^{-2}$. Four additional scans were made at $\lambda=1400 \mathrm{~nm}, \lambda=1450 \mathrm{~nm}, \lambda=1500 \mathrm{~nm}$, and at $\lambda=1550$ $\mathrm{nm}$. The resulting deduced three photon absorption coefficients are plotted versus frequency in Fig. C.7. We observe that $\gamma$ tends to zero as the frequency approaches 
the $\frac{1}{3} E_{\text {gap }}$, confirming a three-photon absorption process. A-three photon process could be used to homogeneously switch GaP photonic crystals, such as the one shown in Fig.1.3D.

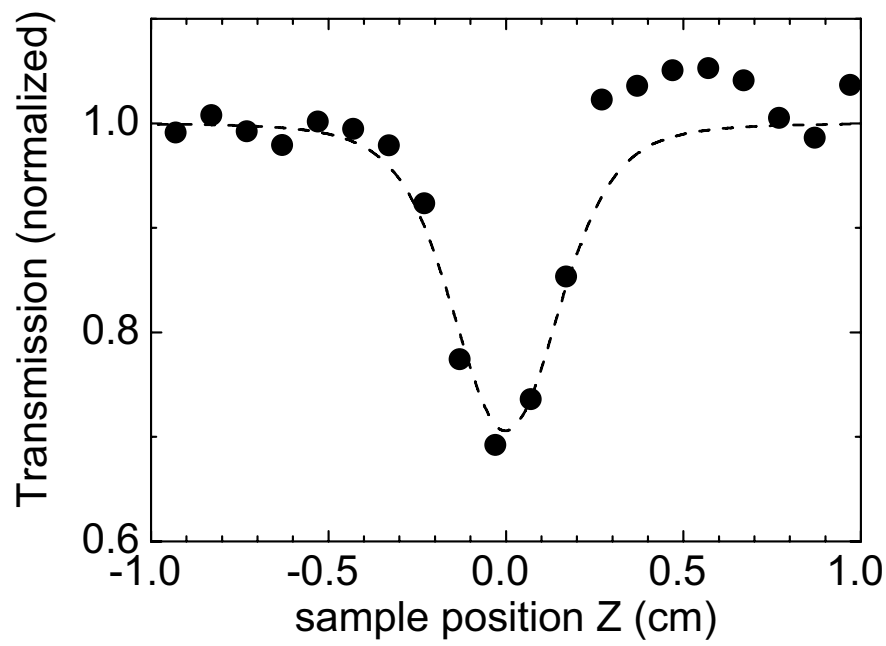

Figure C.6: Open aperture z-scan measurement for a $300 \mu \mathrm{m}$ thick double-side polished GaP wafer. Pump parameters: $\lambda=1600 \mathrm{~nm}, f=100 \mathrm{~mm}, \tau_{p}=130 \mathrm{fs}_{\mathrm{s}} \mathrm{I}_{0}=285 \pm 60 \mathrm{GWcm}^{-2}$. The curve represents the calculated transmission using a three photon coefficient $\gamma=1.0 \pm 0.3 \times 10^{-3}$

\section{C.3 Conclusion}

We have measured for the first time the two-photon absorption coefficients of GaAs and of Si near the two-photon absorption edge by an open-aperture z-scan technique. The experimental data was compared to a model that includes nonlinear absorption in the sample. For both $\mathrm{Si}$ and GaAs we find that the two-photon absorption coefficient tends to zero near half the gap energy $\frac{1}{2} E_{\text {gap }}$. The value of $\beta=0.6 \pm 0.3 \mathrm{cmGW}^{-1}$ at $\lambda=1630 \mathrm{~nm}$ is in agreement with data obtained by Ref. [97] at slightly shorter wavelength and at much lower pump irradiance: $\beta=0.79 \pm 0.12$ $\mathrm{cmGW}^{-1}$ at $\lambda=1550 \mathrm{~nm}$.

Three-photon absorption coefficients $\gamma$ for gallium phosphide were deduced at a range of pump wavelengths in the three-photon absorption range $\lambda=1400-1600$ $\mathrm{nm}$. We observe that $\gamma$ tends to zero as the frequency approaches the $\frac{1}{3} E_{\text {gap }}$, in agreement with a three-photon process. We also observe an effect of Kerr lensing, and conclude that the Kerr coefficient of $\mathrm{GaP}$ attains a positive sign in this frequency 


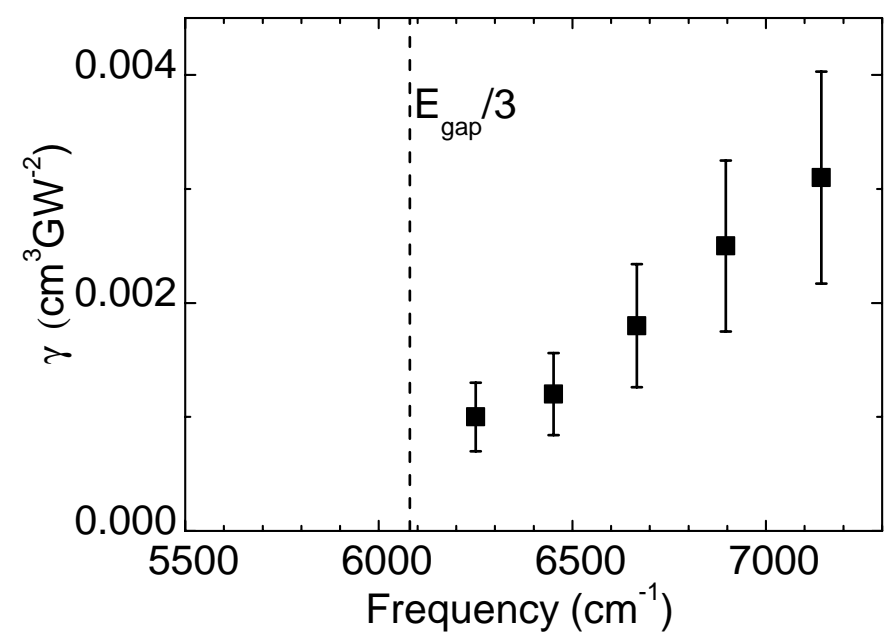

Figure C.7: Three photon coefficient $\gamma$ for GaP were obtained at five wavelengths. The dashed vertical line the three-photon absorption edge for $\mathrm{GaP} \frac{1}{3} E_{\text {gap }}$. We observe that $\gamma$ decreases as the pump frequency approaches $\frac{1}{3} E_{\text {gap }}$.

range. We propose that the observed opposite sign of the refractive index change by free-carrier effects and the change due to nonlinear refraction in $\mathrm{GaP}$ allows for non-monotonic switching experiments in GaP photonic crystals. 



\section{Samenvatting}

Er is wereldwijd een sterk groeiende interesse voor materialen waarmee de emissie en propagatie van fotonen kan worden beheerst en waarmee licht kan worden gevangen. Bij uitstek daartoe geschikt zijn metamaterialen; dit zijn materialen waarin twee of meer verschillende stoffen in een specifiek ruimtelijk patroon nieuwe optische eigenschappen hebben die niet voorkomen in de afzonderlijke stoffen. Dit proefschrift richt zich speciaal op fotonische kristallen, een speciaal soort metamaterialen die de toegang kunnen verbieden aan licht met bepaalde kleuren.

Fotonische kristallen zijn periodieke structuren die opgebouwd zijn uit twee materialen met verschillende brekingsindices. Aan de grensoppervlakken waar het ene materiaal overgaat in het andere materiaal worden lichtgolven verstrooid. Wanneer de periodieke variatie van de brekingsindex plaatsvindt op lengteschalen die vergelijkbaar zijn met de golflengte van licht, dan kan er interferentie van de verstrooide lichtgolven optreden. Het verschijnsel waarbij constructieve interferentie van verstrooide golven een verhoogde reflectie veroorzaakt in een bepaalde richting wordt optische Bragg diffractie genoemd. Het frequentiebereik waarin propagatie van licht verboden is wordt een stopgap genoemd. De gemeten reflectiepiek wordt een stopband genoemd.

Hoe groter het brekingsindex verschil tussen de beide materialen in het kristal wordt, hoe sterker de interactie met licht wordt en hoe breder de stopgaps. In driedimensionale fotonische kristallen kan dan zelfs een frequentiegebied ontstaan waarin stopgaps in alle richtingen overlappen, zodat licht zich in geen enkele richting kan voortplanten. Zo'n gebied wordt een fotonische bandkloof genoemd. Voor licht met een frequentie die in de bandkloof ligt, zijn in 1987 twee belangrijke fundamentele effecten voorspeld. Ten eerste kan spontane emissie van lichtbronnen 
volledig worden stilgezet doordat een photon niet kan worden uitgezonden. Ten tweede is voorspeld dat het mogelijk is om licht in de buurt van een defect in een fotonisch kristal stil te zetten. De frequentie-positie van de bandkloof van een kristal ligt vast door de rangschikking en de brekingsindex van de gebruikte materialen; als een kristal eenmaal gemaakt is, verandert de bandkloof in principe niet meer.

Het ligt voor de hand dat de potentiële mogelijkheden van zo'n bandkloof veel groter zijn, als de bandkloof in de tijd gecontroleerd veranderd kan worden. Dit is vooral interessant wanneer de verandering ultrasnel plaatsvindt. De bandkloof van een kristal kan geschakeld worden door bijvoorbeeld de brekingsindex van één van de componenten te wijzigen. Voor halfgeleiders die voldoende hoge brekingsindex hebben om een fotonische bandkloof te realiseren, blijkt dat de brekingsindex tijdelijk en ultrasnel verminderd kan worden door met een lichtpuls elektronen in de halfgeleider aan te slaan. Met korte en intense laserpulsen kan de brekingsindex voldoende veranderd worden om de bandkloof van kleur te laten verschuiven. Men zou daarmee bijvoorbeeld een aangeslagen natrium atoom in een kristal met een bandkloof voor oranje licht opeens met de schakelpuls tot spontane emissie kunnen laten overgaan. Hierbij zorgt de schakelpuls ervoor dat de bandkloof ineens niet meer oranje licht verbiedt, maar blauwere kleuren. Dit proefschrift beschrijft de eerste schakelexperimenten fotonische bandkloof kristallen, waarin de frequentiepositie van de stopband verandert door het optisch aanslaan van elektronen.

In Hoofstuk 1 worden enkele belangrijke doelen van het experiment gesteld zoals het schakelen van spontane emissie en het schakelen van de groepssnelheid van licht, waardoor pulsen stilgezet kunnen worden. Het hoofdstuk bevat ook een korte discussie over de tijdschaal waarop de schakeling plaats moet vinden zodat deze niet meer adiabatisch is.

Vier belangrijke eisen waar schakelexperimenten aan fotonische kristallen aan moeten voldoen worden besproken in Hoofdstuk 2. Ten eerste moet de verandering in brekingsindex moet groot genoeg zijn. Ten tweede moet de verandering snel plaatsvinden. Ten derde is het ook relevant dat het geschakelde kristal niet absorberend wordt. Ten vierde is het belangrijk dat de schakelpuls niet alleen een dunne oppervlakte laag van het kristal schakelt, maar doordringt in een groter volume. In Hoofdstuk 3 wordt uitgelegd hoe in schakelexperimenten aan deze vier eisen kan worden voldaan. Uit onze analyse blijkt blijkt dat de ruimtelijke homogeniteit van geschakelde halfgeleiders en fotonische kristallen kan worden geoptimaliseerd door een twee-foton absorptie proces te gebruiken in plaats van lineaire absorptie. De pomp en probe opstelling die gebruikt is in de schakelmetin- 
gen wordt in detail beschreven in Hoofdstuk 4. Een belangrijke eigenschap van de opstelling is dat zowel de schakelpuls als de meetpuls over een breed spectrum aan kleuren is te verstemmen, waardoor octaaf-brede tijdsopgeloste reflectiemetingen mogelijk zijn. Verder wordt er aandacht besteed aan een nieuwe meetmethode waarmee ruis tengevolge van laserfluctuaties sterk kan worden onderdrukt.

Hoofdstuk 5 beschrijft schakelmetingen aan GaAs/AlAs Bragg reflectoren. Deze eendimensionale samples zijn uitermate geschikt om het schakelproces in detail te bestuderen, omdat de structuur een vergelijking met exacte transfer matrix theorie toestaat. We gebruiken twee-foton absorbtie om selektief elektronen aan te slaan in de GaAs lagen van de structuur en oberveren zowel een ultrasnelle versmalling als een opschuiving van de reflectiepiek. Het schakelproces gaat gepaard met grote veranderingen tot $\Delta \mathrm{R}>40 \%$ in de reflectiviteit aan de randen van de stopband. De geschakelde spectra komen goed overeen met transfer matrix theorie waarin het effect van de geëxciteerde elektronen wordt beschreven met het Drude model.

Hoofstuk 6 laat de eerste metingen zien aan fotonische bandkloof structuren. De silicium houtstapel kristallen bestaan uit regelmatig opgestapelde nanobalkjes van silicium. Samen vormen deze balkjes een diamantachtige kristal structuur. Reflectiemetingen in het frequentiegebied van de bandkloof vertonen een brede stopband met een hoge piek reflectiviteit van bijna $95 \%$. De kristallen worden homogeen aangeslagen via een twee-foton proces en de geschakelde reflectiviteit wordt gemeten over een octaaf-breed frequentie gebied. Op zeer korte korte tijden $<200 \mathrm{fs}$ nemen we een verrassende vernauwing van de stop band waar, terwijl op "langere"tijden, na ongeveer een picoseconde, de complete stopband verschuift naar hogere frequentie. Dit niet-monotone gedrag wordt veroorzaakt door een combinatie van een Kerr effect op korte tijden en het effect van de vrijgemaakte elektronen op iets langere tijden. De gemeten reflectiespectra komen goed overeen met exact modal method berekeningen waarin het effect van de elektronen wordt beschreven met het Drudemodel. Op grond van onze experimenten voorspellen we interessante effecten in de LDOS op korte tijdschalen.

Schakelmetingen aan silicium inverse opalen worden gepresenteerd in Hoofdstuk 7. In de kristallen worden met behulp van twee-foton absorbtie elektronen vrijgemaakt. De reflectiviteit van de kristallen wordt gemeten in het gebied van tweede orde Bragg verstrooing; het gebied waar een bandkloof is voorspeld. We observeren een grote verschuiving van alle pieken naar hogere frequenties. We vergelijken de geschakelde spectra met berekende bandenstructuren. We herleiden een grote maximale verandering van de brekingsindex van $\Delta n_{S i}^{\prime} / n_{S i}^{\prime}>2 \%$. We herleiden voor de eerste keer een DOS verandering uit experimentele schakelmetingen. De verandering van de toestandsdichtheid is naar schatting groot genoeg om 
de spontane emissie levensduur van lichtbronnen te schakelen.

In Hoofdstuk 8 worden enkele aanbevelingen gegeven voor verdere experimenten. Ten eerste laten we zien dat in experimenten met trilholtes met een nauwe resonantie de benodigde energie in de schakelpuls sterk kan worden verlaagd tot $50 \mathrm{fJ}$, hetgeen belangrijk is voor mogelijke toepassingen. Ten tweede stellen we een experiment voor waarin de overgang tussen het adiabatische en niet-adiabatische regiem kan worden onderzocht. Ten derde stellen we een methode voor om de spontante emissie veranderingen in geschakelde kristallen te meten met behulp van utrasnelle sluiters. Ten vierde adviseren we het verrichten van metingen waarin ook de pump frequentie wordt verandert om het schakelgedrag op $t=0 \mathrm{fs}$ in de houtstapel kristallen te onderzoeken. Ten vijfde stellen voor om de twee-foton absorbtie coefficient van onze kristallen te bepalen met behulp van niet fotonische referentie samples. Als laatste stellen we voor om schakelmetingen te doen aan fotonische kristalllen die gemaakt zijn met behulp van ets technieken. 


\section{Dankwoord}

In de eerste plaats bedank ik mijn promotor. Willem, ik ben je dankbaar voor de grote mate van vrijheid en vertrouwen die je me schonk. Ik heb genoten van de bewegingsruimte die je me gunde tijdens het opbouwen van ons experiment. Tegelijkertijd was het erg fijn om je altijd als coach aan de zijlijn te hebben die op het juiste moment de speelwijze aanpast.

Nog meer wil ik je bedanken voor de vele dingen die je me geleerd hebt, zoals het overzicht proberen te houden in een project; "wat is het doel?" maar ook het tot in details analyseren van data om zo tot nieuwe inzichten te komen. Verder leerde je me om resultaten meer didactisch te presenteren en om kleine successen te waarderen als de grote nog even uitblijven. Je gedrevenheid en passie voor natuurkunde hebben aanstekelijk op me gewerkt. Ik waardeer de steun en adviezen die je me op persoonlijk vlak hebt gegeven.

Ad, ik heb het erg leerzaam gevonden om met je samen te werken. Bedankt voor de wetenschappelijke discussies, waarin je scherpe blik en nieuwe ideeën inspirerend hebben gewerkt. Ik ben je stijl van leiding geven gaan waarderen omdat hierin het belang van het onderzoek voorop blijft staan, zelfs als hiervoor moeilijke beslissingen nodig zijn. Bedankt voor je opbouwende kritiek tijdens werkbesprekingen en colloquia, waarvan ik veel heb geleerd. Verder dank ik je voor de keren dat je me persoonlijk advies hebt gegeven.

Cock, zonder jouw vakkundige technische ondersteuning zouden experimenten in het COPS-lab nooit zo voorspoedig zijn verlopen. Welk probleem er ook was, je had altijd een geschikte oplossing paraat. Ik heb met plezier met je samengewerkt, in het bijzonder herinner ik me ons flitsende wit-licht avontuur in Denemarken. In de onzekere periode waarin een groot deel van de groep naar Amsterdam vertrok, heb je je ondanks alles volledig ingezet om alles in goed banen te leiden. 
Het was fijn om op AMOLF in Rob K. dezelfde deskundigheid en ervaring terug te vinden. Je hebt erg veel voor onze experimentele opstellingen gedaan, in het bijzonder in de periode van de verhuizing, waar je ons aanspreekpunt bent geweest naar de technische staf op AMOLF. Het is mede dankzij jouw inzet geweest dat, ondanks onvoorziene problemen, na enkele maanden de eerste schakelmetingen op AMOLF konden worden gedaan.

Adriaan, ik wil je bedanken voor de fijne samenwerking. Je hebt tijdens je afstudeerproject een bijzondere prestatie geleverd. Je kreeg een complexe opstelling snel onder de knie. De schakelmetingen die je ermee hebt verricht aan de woodpile kristallen waren monnikenwerk, iets wat jou goed lag. Je goed georganiseerde werkmethode heeft ervoor gezorgd dat de experimenten zo goed zijn verlopen. Voor de wetenschap is het jammer dat jouw doel ergens anders ligt.

Rob v. L., ik kijk terug op een leuke tijd waarin we samen met Léon aan cavities in opalen hebben gewerkt. Hoewel je niet aan het schakelexperiment werkte, heeft je mooie werk wel de basis gevormd voor verdere experimenten binnen de groep.

Zonder een goede computer ondersteuning was dit onderzoek niet mogelijk geweest. Op dit gebied wil ik twee mensen speciaal bedanken die mij verschillende malen hebben geholpen in tijden van nood: Bas in Twente en Richard op AMOLF. Voor advies op het gebied van elektronica wil ik Frans en Rindert bedanken.

Albert, ik wil je hartelijk bedanken voor het genereus ter beschikking stellen van de prachtige woodpile samples en Si inverse opalen. Jeroen, het was erg leuk om samen met je te kunnen meten aan en te discussiëren over de Si inverse opalen. I would like to thank you, Jim Fleming, and David Norris for the beautiful photonic crystals that are discussed in Chapter 6 and 7 respectively.

Femius, ik bedank je voor je geduldige uitleg over fotonische kristallen in het begin van mijn promotie, en voor de inzichten die je hebt me hebt gegeven. Verder bedank ik je voor je hulp bij het interpreteren van bandenstructuur berekeningen aan inverse opalen. Ik wil Allard bedanken voor zijn hulp bij het analyseren van onze data en voor zijn interessante ideeën voor nieuwe experimenten.

Ik wil Henry van Driel bedanken voor de prettige samenwerking en voor zijn deskundige hulp bij de interpretatie van onze data aan GaAs/AlAs structuren. In Tampere, I would like to thank Soile Suomalainen and Mircea Guina for our collaboration and for the excellent III-V Bragg stacks.

Léon, Willem T. en Frans; de drie ets- en fibkoningen uit het oosten; helaas ga ik iets te vroeg weg om jullie prachtige fotonische structuren te kunnen schakelen. Ik bewonder jullie gedrevenheid en doorzettingsvermogen in het systematisch verbeteren van de zeer uitdagende fabricagetechnieken van fotonische kristallen. En het spijt me nog steeds van dat ene "permanent geschakelde"kristal. . 
Op AMOLF wil ik graag de groepsleiders van het Center for Nanophotonics bedanken voor hun initiatieven om samenhang en informatieoverdracht tussen de groepen te stimuleren. De wekelijkse colloquia en de nanofotonica workshop op Ameland zijn voorbeelden hiervan die ik als erg nuttig heb ervaren. De kritische, maar enthousiaste manier waarop tijdens de colloquia en werkbesprekingen over wetenschap wordt gediscussiëerd spreekt me erg aan. Ik bedank iedereen die een actieve bijdrage heeft geleverd aan dit motiverende klimaat, in het bijzonder de groepsleiders.

Voor het regelen van vele administratieve zaken wil ik in Twente Karen en Raymond bedanken en Ed voor zijn hulp bij vele praktische zaken op AMOLF.

Als experimentator neem ik op deze plek ook een beetje afscheid van "mijn" opstelling. Een troostende gedachte is wel dat de experimenten die we zijn begonnen voortgezet worden. Philip, ik wens je veel succes met de mooie schakelexperimenten die in het vooruitzicht liggen.

Zoals wellicht bekend ben ik halverwege mijn promotie samen met een deel van onze groep verhuisd van Enschede naar Amsterdam. Ik kijk met enigzins gemengde gevoelens terug op het vertrek uit Twente. In Amsterdam lagen weliswaar vele nieuwe kansen en uitdagingen te wachten, maar achteraf gezien heeft de verhuizing ook de opsplitsing betekend van de bijzonder hechte Complex Photonic Systems groep zoals die toen was.

Voor hun hulp tijdens de verhuizing naar Amsterdam wil ik op AMOLF Piet, Wouter en Roudy bedanken. Ik bedank ook alle groepsgenoten die hebben geholpen met het zorgvuldig inpakken van ieder onderdeeltje van de experimentele opstellingen.

Een van de voordelen van het experimenteren op AMOLF die ik al snel ontdekte was dat je er nooit alleen aan het werk bent; niet als je experiment uitloopt tot in de kleine uurtjes en ook niet in als er in het weekend nog wat moet worden gemeten. Ik wil daarom de "vaste" overwerkers Alex, Avi, Dima, Euan, Jord, Maria, Marijn, Paolo, Rob E. en Willem bedanken voor de gezelligheid en ook voor het letten op elkaars veiligheid.

Niet alleen op wetenschappelijk gebied heb ik een leuke promotie-tijd gehad. Zowel in Twente als op AMOLF is er een fijne werksfeer. Zonder uitzondering heb ik met iedereen goed op kunnen schieten. De leuke sfeer wordt voor een belangrijk deel bepaald door gezellige buitenlabse activiteiten zoals: etentjes, housewarmings, voetbalpartijen, barbecues, borrels, spelavonden, filmavonden, zwemmiddagen en Batavierenrace trainingen. Zonder in een opsomming van namen te vervallen wil ik iedereen bedanken die in de afgelopen jaren hieraan heeft meegedaan. 
Ik wil een paar mensen speciaal bedanken waarmee ik door de jaren heen persoonlijk een sterke band heb gekregen. Alex, Cock, Dima, Femius, Ivan, Ivo, Jaime, Karen, Léon, Martijn, Paolo, Peter, Raymond, Tom, Willem en Willem T. bedankt voor de vele leuke momenten en voor jullie vriendschap.

Tom, het was erg fijn om in de afgelopen jaren de ups en downs binnen en buiten onze promoties met jou te kunnen delen. Ik heb genoten van de keren dat we samen hebben gekookt, gedronken en gesport. Ook was het fijn om af en toe lekker Hollands te mopperen over dingen waaraan we ons gezamenlijk ergerden, niet in de laatste plaats tijdens onze verkenning van de Amsterdamse woningmarkt.

Ivan, jouw nuchtere kijk op dingen werkt verhelderend. Ik bedank je voor je adviezen die mij vaak hebben geholpen. Ik wil jou en Nina ook bedanken voor jullie vriendschap en de gezellige avonden in het Amsterdamse. Ik wens jullie veel geluk in Brabant.

Mika, ik wil je een plaats geven in dit dankwoord omdat je erg belangrijk voor me bent geweest. Ons avontuur verliep niet zoals we hadden gehoopt, maar we kunnen in ieder geval terugkijken op een mooie tijd.

Hidde en Wenneke, wat is het fijn om je bij mensen thuis te voelen. Ik heb veel steun en advies van jullie gekregen op momenten dat dat nodig was. Jullie zijn geweldige vrienden.

Ik bedank verder alle vrienden en familieleden die me in de afgelopen jaren hebben bijgestaan, raad hebben gegeven of gewoon interesse hebben getoond in waar ik mee bezig was. Ik bedank in het bijzonder mijn paranimfen Tamar en Sjoerd. Lieve zus en broer, bedankt voor jullie steun en voor het in goede banen leiden van mijn promotiedag.

Lieve mama en papa, jullie hebben onvoorwaardelijk achter me gestaan in de keuzes die ik door de jaren heen heb gemaakt. Ik wil jullie bedanken voor jullie liefde en steun. Dit proefschrift draag ik op aan jullie. 


\section{References}

[1] C.M. Soukoulis, editor, Photonic crystals and light localization in the 21st century, Kluwer, Dordrecht, 2001. - p.13.

[2] F. Bloch, Z. f. Physik 52, 555 (1928). - p.13.

[3] N. W. Ashcroft and N. Mermin, Solid State Physics, Thomson Learning, Stamford, 1976. - p.13, 26, 80, 119, and 127.

[4] A. Yariv and P. Yeh, Photonics, optical electronics in modern communications, Oxford university press, New York, 2006. - p.13, 14, and 58.

[5] P. St. J. Russell, "Optical Stop Bands and Photonic Band Gaps: Physics and Applications", in Photonic Band Gap Materials vol. 315 of NATO ASI Ser. E: Applied Sciences, ed. by C.M. Soukoulis, 1996. - p.13.

[6] E. Yablonovitch, Phys. Rev. Lett. 58(20), 2059 (1987). — p.13 and 17.

[7] S. John, Phys. Rev. Lett. 58(23), 2486 (1987). — p.13.

[8] P. Lodahl, A. F. van Driel, I. Nikolaev, A. Irman, K. Overgaag, D. Vanmaekelbergh, and W. L. Vos, Nature 430, 654 (2004). — p.13, 18, and 100.

[9] A. Kress, F. Hofbauer, N. Reinelt, M. Kaniber, H. Krenner, J. Meyer, G. Böhm, and J. Finley, Phys. Rev. B 71, 241304 (2005). - p.13.

[10] D. Englund, D. Fattal, E. Waks, G. Solomon, B. Zhang, T. Nakaoka, Y. Arakawa, Y. Yamamoto, and J. Vučković, Phys. Rev. Lett. 95, 013904 (2005). - p.13.

[11] M. Fujita, S. Takahashi, Y. Tanaka, T. Asano, and S. Noda, Science 308, 1296 (2005). - p.13.

[12] E. Yablonovitch, T. J. Gmitter, R. D. Meade, A. M. Rappe, K. D. Brommer, and J. D. Joannopoulos, Phys. Rev. Lett. 67, 3380 (1991). — p.13.

[13] R. W. James, The optical principles of the diffraction of $x$-rays, G. Bell and Sons, London, 1958. - p.14.

[14] W. L. Vos, R. Sprik, A. van Blaaderen, A. Imhof, A. Lagendijk, and G. H. Wegdam, Phys. Rev. B 53, 16231 (1996). - p.15, 58, 80, and 81.

[15] W. L. Vos, H. M. van Driel, M. Megens, A. F. Koenderink, and A. Imhof, in Photonic crystals and light localization in the 21st century, edited by C. M. Soukoulis, Kluwer, Dordrecht, 2001. - p.15 and 58.

[16] A. F. Koenderink, Emission and transport of Light in Photonic Crystals, PhD thesis, University of Amsterdam, 2003. - p.15, 99, 107, and 120.

[17] A. F. Koenderink, A. Lagendijk, and W. L. Vos, Phys. Rev. B 72, 153102 (2005). - p.16, 17, 40, and 76 .

[18] E. Yablonovitch and T. J. Gmitter, Phys. Rev. Lett. 63, 1950 (1989). — p.17.

[19] K. M. Leung and Y. F. Liu, Phys. Rev. Lett. 65, 2446 (1990). — p.17. 
[20] Z. Zhang and S. Satpathy, Phys. Rev. Lett. 65, 2650 (1990). - p.17.

[21] H. S. Sözüer, J. W. Haus, and R. Inguva, Phys. Rev. B 45, 13962 (1992). — p.17 and 99.

[22] K. M. Ho, C. T. Chan, C. M. Soukoulis, R. Biswas, and M. Sigalas, Solid State Comm. 89, 413 (1994). - p.17, 74, and 79.

[23] E. Özbay, E. Michel, G. Tuttle, R. Biswas, K.-M. Ho, J. Bostak, and D. M. Bloom, Appl. Phys. Lett. 65, 1617 (1994). — p.17.

[24] S. Y. Lin, J. G. Fleming, D. L. Hetherington, B. K. Smith, R. Biswas, K. M. Ho, M. M. Sigalas, W. Zubrzycki, S. R. Kurtz, and J. Bur, Nature 394, 251 (1998). - p.17 and 96 .

[25] J. G. Fleming and S.-. Y. Lin, Opt. Lett. 24, 49 (1999). - p.17, 41, and 74.

[26] S. Noda, K. Tomoda, N. Yamamoto, and A. Chutinan, Nature 289, 604 (2000). - p.17 and 80.

[27] S. Hachisu and S. Yoshimura, Nature 283, 188 (1980). - p.17.

[28] J. V. Sanders, Nature 204, 1151 (1964). — p.17.

[29] B. T. Holland, C. F. Blanford, and A. Stein, Science 281, 5376 (1998). - p.17. [30] J. E. G. J. Wijnhoven and W. L. Vos, Science 281, 802 (1998). — p.17 and 99.

[31] A. A. Zakhidov, R. H. Baughman, Z. Iqbal, C. Cui, I. Khayrullin, S. O. Dantas, J. Marti, and V. G. Ralchenko, Science 282, 897 (1998). — p.17.

[32] W. L. Vos and H. M. van Driel, Phys. Lett. A 272, 101 (2000). — p.17, 25, and 99.

[33] A. Blanco, E. Chomski, S. Grabtchak, M. Ibisate, S. John, S. W. Leonard, C. López, F. Meseguer, H. Míguez, J. P. Mondia, G. A. Ozin, O. Toader, and H. M. van Driel, Nature 405, 437 (2000). - p.18, 74, 99, and 106.

[34] Y. A. Vlasov, X.-Z. Bo, J. C. Sturm, and D. J. Norris, Nature 414, 289 (2001). - p.18, 74, 99, 100, and 109.

[35] E. Palacios-Lidon, A. Blanco, M. Ibisate, F. Meseguer, and C. López, Appl. Phys. Lett. 81, 4925 (2002). - p.18, 99, 104, 107, and 109.

[36] P. Jiang, J. F. Bertone, and V. L. Colvin, Science 291, 453 (2001). — p.18.

[37] S. Gottardo, M. Burresi, F. Geobaldo, L. Pallavidino, F. Giorgis, and D. S. Wiersma, Phys. Rev. E. 74, 040702 (2006). — p.18 and 109.

[38] A. van Blaaderen, R. Ruel, and P. Wiltzius, Nature 385, 321 (1997). - p.18.

[39] J. P. Hoogenboom, A. K. Langen-Suurling, J. Romijn, and A. van Blaaderen, Phys. Rev. Lett. 90, 138301 (2002). - p.18.

[40] T. G. Euser, A game of $\mu$-golf: Growth and analysis of colloidal crystals on 2D structures fabricated with laser interference lithography, Master's thesis, University of Twente, 2002. - p.18 and 19.

[41] N. V. Dziomkina, M. A. Hempenius, and G. J. Vancso, Adv. Mater. 17, 237 
(2005). - p.18.

[42] L. Vogelaar, W. Nijdam, H. A. G. M. van Wolferen, R. M. de Ridder, F. B. Segerink, E. Fluck, L. Kuipers, and N. F. van Hulst, Adv. Mater. 13(20), 1551 (2001). - p.18.

[43] L. Prodan, T. G. Euser, H. A. G. M. van Wolferen, C. Bostan, R. D. de Ridder, R. Beigang, K.-J. Boller, and L. Kuipers, Nanotechnology 15, 639 (2004). - p.18.

[44] M. Campbell, D. N. Sharp, M. T. Harrison, R. G. Denning, and A. J. Turberfield, Nature 404, 53 (2000). - p.18.

[45] S. Yang, M. Megens, J. Aizenberg, P. Wiltzius, P. M. Chaikin, and W. B. Russel, Chem. Mater. 14, 2831 (2002). - p.18.

[46] Y. Miklyaev, D. Meisel, A. Blanco, G. von Freymann, K. Busch, W. Koch, C. Enkrich, M. Deubel, and M. Wegener, Appl. Phys. Lett. 82, 1284 (2003). - p.18.

[47] N. Tétreault, G. von Freymann, M. Deubel, M. Hermatschweiler, F. PérezWillard, S. John, M. Wegener, and G. A. Ozin, Adv. Mater. 18, 457 (2005). - p.18.

[48] M. Deubel, G. von Freymann, M. Wegener, S. Pereira, K. Busch, and C. Soukoulis, Nat. Mater. 3, 444 (2004). - p.18.

[49] M. Deubel, M. Wegener, S. Linden, G. von Freymann, and S. John, Opt. Lett. 31, 805 (2006). - p.18.

[50] S. Wong, M. Deubel, F. Pérez-Willard, S. John, G. r21.00g(S.)0nTJF189.963Tf0-14.346Tc 
J. Bloch, Phys. Rev. Lett. 95, 067401 (2005). — p.19.

[59] H. Kosaka, T. Kawashima, A. Tomita, M. Notomi, T. Tamamura, T. Sato, and S. Kawakami, Phys. Rev. B 58, 10099 (R) (1998). — p.20.

[60] A. F. Koenderink and L. Vos, W, Phys. Rev. Lett. 91, 213902 (2003). — p.20.

[61] H. Gersen, T. Karle, R. J. P. Engelen, W. Bogaerts, J. P. Korterik, N. F. van Hulst, T. Krauss, and L. Kuipers, Phys. Rev. Lett. 92, 083901 (2004). — p.20.

[62] R. J. P. Engelen, Y. Sugimoto, Y. Watanabe, J. P. Korterik, N. Ikeda, N. F. van Hulst, K. Asakawa, and L. . Kuipers, Opt. Express 14, 1658 (2006). - p.20.

[63] B. P. Bret, T. L. Sonnemans, and T. W. Hijmans, Phys. Rev. A 68, 023807 (2003). - p.20 and 124.

[64] M. F. Yanik and S. Fan, Phys. Rev. Lett. 92, 083901 (2004). - p.20 and 73.

[65] A. Liu, R. Jones, L. Liao, D. Samara-Rubio, D. Rubin, O. Cohen, R. Nicolaescu, and M. Paniccia, Nature 427, 615 (2004). - p.20 and 24.

[66] A. Hache and M. Bourgeois, Appl. Phys. Lett. 77 (2000). — p.20, 25, and 74.

[67] S. R. Hastings, M. J. A. de Dood, H. Kim, W. Marshall, H. S. Eisenberg, and D. Bouwmeester, Appl. Phys. Lett. 86, 031109 (2005). - p.20, 25, 35, 66, and 74 .

[68] S. W. Leonard, H. M. van Driel, J. Schilling, and R. B. Wehrspohn, Phys. Rev. B 66, 161102 (R) (2002). - p.20, 25, 30, 35, 74, and 129.

[69] A. D. Bristow, J. P. R. Wells, W. H. Fan, A. M. Fox, M. S. Skolnick, D. M. Whittaker, A. Tahraoui, T. F. Krauss, and J. S. Roberts, Appl. Phys. Lett 83, 851 (2003). - p.20 and 74.

[70] M. Born and V. Fock, Z. Phys. 51, 165 (1928). - p.21.

[71] We thank Allard Mosk for this suggestion. - p.21 and 125.

[72] J. N. Winn, S. Fan, and J. D. Joannopoulos, Phys. Rev. B. 59, 1551 (1999). - p.21.

[73] K. Sokolowski-Tinten and D. von der Linde, Phys. Rev. B. 61, 2643 (2000). - p.23, 27, 39, 119, 127, 129, and 131.

[74] V. R. Almeida, C. A. Barrios, P. R. R., and M. Lipson, Nature 431, 1081 (2004). - p.24.

[75] J. M. Weissman, H. B. Sunkara, A. S. Tse, and S. A. Asher, Science 274, 959 (1996). - p.24.

[76] K. Yoshino, Y. Kawagishi, M. Ozaki, and A. Kose, Jpn. J. Appl. Phys. 38, L786 (1999). - p.24.

[77] A. F. Koenderink, M. Kafesaki, B. C. Buchler, and V. Sandoghdar, Phys. Rev. Lett. 95, 153904 (2005). - p.24.

[78] I. Märki, M. Salt, and H. P. Herzig, Opt. Express 7, 2969 (2006). - p.24.

[79] W. C. L. Hopman, A. J. F. Hollink, R. M. de Ridder RM, K. O. van der Werf, 
V. Subramaniam, and W. Bogaerts, Opt. Express 14, 8745 (2006). - p.24.

[80] A. Figotin and Y. A. Godin, Phys. Rev. B 57, 2841 (1998). — p.24.

[81] K. Busch and S. John, Phys. Rev. Lett. 83, 967 (1999). — p.24.

[82] K. Yoshino, Y. Shimoda, Y. Kawagishi, K. Nakayama, and M. Ozaki, Appl. Phys. Lett. 75, 932 (1999). - p.24.

[83] S. W. Leonard, J. P. Mondia, H. M. van Driel, O. Toader, and S. John, Phys. Rev. B 61, 2389 (R) (2000). - p.24.

[84] D. Kang, J. E. Maclennan, N. A. Clark, A. A. Zakhidov, and R. H. Baughman, Phys. Rev. Lett. 86, 4052 (2001). - p.24.

[85] Y. Shimoda, M. Ozaki, and K. Yoshino, Appl. Phys. Lett. 79, 3627 (2001). - p.24.

[86] S. Gottardo, D. S. Wiersma, and W. L. Vos, Physica B 338, 143 (2003). - p.24.

[87] V. G. Golubev, V. Y. Davydov, N. F. Kartenko, D. A. Kurdyukov, V. A. Medvedev, A. B. Pevtsov, A. V. Scherbakov, and E. B. Shadrin, Appl. Phys. Lett. 79, 2127 (2001). - p.24.

[88] D. A. Mazurenko, R. Kerst, J. I. Dijkhuis, A. V. Akimov, V. G. Golubev, A. A. Kaplyanskii, D. A. Kurdyukov, and A. B. Pevtsov, Appl. Phys. Lett. 86, 041114 (2005). - p.24 and 91.

[89] A. V. Scherbakov, A. V. Akimov, V. G. Golubev, A. A. Kaplyanskii, D. A. Kurdyukov, A. A. Meluchev, and A. B. Pevtsov, Physica E 17, 429 (2003). - p.24.

[90] D. A. Mazurenko, R. Kerst, J. I. Dijkhuis, A. V. Akimov, V. G. Golubev, D. A. Kurdyukov, A. B. Pevtsov, and A. V. Sel'kin, Phys. Rev. Lett. 91, 213903 (2003). - p.25, 35, 74, 91, and 100 .

[91] C. Becker, S. Linden, G. von Freymann, M. Wegener, N. Tétreault, E. Vekris, V. Kitaev, and G. A. Ozin, Appl. Phys. Lett. 87, 091111 (2005). — p.25, 74, 91, 100, 129, and 131.

[92] T. G. Euser, M. Guina, S. Suomalainen, J. Kalkman, A. Polman, Y. Jun, H. Wei, D. J. Norris, and W. L. Vos, in presented at CLEO/QELS Meeting (QTuC1), 2006. - p.25.

[93] T. G. Euser, A. J. Molenaar, J. G. Fleming, B. Gralak, A. Polman, and W. L. Vos, http://arxiv.org/abs/physics/0603045 . - p.25.

[94] P. Drude, Annalen der Physik 1, 566 (1900). - p.26, 119, and 127.

[95] S. J. Kole, PhD thesis, Rijksuniversiteit Groningen, 2003. - p.30.

[96] E. D. Palik, editor, Handbook of Optical Constants of Solids, Academic Press Inc., London, 1985. - p.31, 32, 52, 58, 60, 61, 66, 74, 82, 83, and 109.

[97] M. Dinu, F. Quochi, and H. Garcia, Appl. Phys. Lett. 82, 2954 (2003). — p.31, $32,35,37,92,93,140,144$, and 146 . 
[98] J. F. Reintjes and J. C. McGroddy, Phys. Rev. Lett 30, 901 (1973). - p.31 and 32 .

[99] A. J. Sabbah and D. M. Riffe, Phys. Rev. B 66, 165217 (2002). - p.31 and 32.

[100] M. Sheik-Bahae, A. A. Said, T. H. Wei, D. J. Hagan, and E. W. van Stryland, IEEE J. Quantum Electron. 26, 760 (1990). - p.31 and 140.

[101] We thank Martijn Wubs for deriving this equation. - p.31.

[102] A. Dargys and J. Kundrotas, Handbook on physical properties of Ge, Si, GaAs and InP, Science and Encyclopedia Publishers, Vilnius, 1994. - p.35, 37, and 131.

[103] H. van Driel, Appl. Phys. Lett. 44, 617 (1984). - p.35, 91, 120, 128, and 131.

[104] M. Muryama and T. Nakayama, Phys. Rev. B (R) 49, 5737 (1994). — p.37.

[105] K. M. Ho, C. T. Chan, and C. M. Soukoulis, Phys. Rev. Lett. 65, 3152 (1990). - p.41, 74, and 108.

[106] W. Demtröder, Laser Spectroscopy: Basic Concepts and Instrumentation, Springer-Verlag, Berlin, 3 edition, 2002. — p.43 and 141.

[107] A. E. Siegman, Lasers, University Science Books, Mill Valley, USA, 1986. - p.44.

[108] M. Wubs and A. Lagendijk, Phys. Rev. E 65, 046612 (2002). — p.58.

[109] A. Y. Cho and J. R. Arthur, Progress in Solid-State Chemistry 10, 157 (1975). - p.58 and 59.

[110] P. Y. Yu and M. Cardona, Fundamentals of Semiconductors, Springer-Verlag, Berlin, 1996. - p.59, 87, 113, and 130.

[111] J. Kalkman, Controlled spontaneous emission in erbium-doped microphotonic materials, PhD thesis, University of Utrecht, 2005. - p.59, 101, and 102.

[112] A. Y. Cho, Appl. Phys. Lett 19, 467 (1971). — p.59.

[113] M. S. Thijssen, R. Sprik, J. E. G. J. Wijnhoven, M. Megens, T. Narayanan, A. Lagendijk, and W. L. Vos, Phys. Rev. Lett. 83, 2730 (1999). — p.59, 77, and 103.

[114] M. Sheik-Bahae, A. A. Said, T. H. Wei, D. J. Hagan, and E. W. van Stryland, IEEE J. Quantum Electron. 27, 1296 (1991). - p.61 and 140.

[115] M. Born and E. Wolf, Principles of optics (7th edition), Cambridge university press, Cambridge, 1999. - p.69.

[116] S. Adachi, J. Appl. Phys. 58, R1 (1985 1985). — p.69.

[117] T. G. Euser and W. L. Vos, J. Appl. Phys. 97, 043102 (2005). - p.69.

[118] H. M. van Driel, We thank Prof. H. M. van Driel for these insights. - p.69, 128 , and 130.

[119] W. G. Spitzer and J. M. Whelan, Phys. Rev. 114, 59 (1959). — p.69, 70, 130, and 131. 

[120] H. Nakamura, Y. Sugimoto, K. Kanamoto, N. Ikeda, Y. Tanaka, Y. Nakamura, S. Ohkouchi, Y. Watanabe, K. Inoue, H. Ishikawa, and K. Asakawa, Opt. Express 12, 6606 (2004). - p.74.

[121] E. W. van Stryland, H. Vanherzeele, M. A. Woodall, M. J. Soileau, A. L. Smirl, S. Guha, and T. F. J. Boggess, Opt. Eng. 24, 613 (1985). - p.74.

[122] A. Villeneuve, C. C. Yang, P. G. J. Wigley, G. I. Stegeman, J. S. Aitchison, and C. N. Ironside, Appl. Phys. Lett. 61, 147 (1992). - p.74.

[123] C. T. Chan, K. M. Ho, and C. M. Soukoulis, Europhys. Lett. 16, 563 (1991). - p.74.

[124] M. J. A. de Dood, B. Gralak, and A. Polman, Phys. Rev. B 67, 035322 (2003). - p.74, 75, 77, 79, and 94 .

[125] B. Gralak, M. de Dood, G. Tayeb, S. Enoch, and D. Maystre, Phys. Rev. E 67, 066601 (2003). - p.79, 80, 133, 135, 137, and 138.

[126] M. J. A. de Dood, Silicon Photonic Crystals and Spontaneous Emission, PhD thesis, Utrecht University, 2002. - p.79.

[127] A. F. Koenderink, unpublished data. - p.81 and 135.

[128] J. O. White, S. Cuzeau, D. Hulin, and R. Vanderhaghen, J. Appl. Phys. 84, 4984 (1998). - p.87 and 116.

[129] D. M. Whittaker, Opt. Lett. 25, 779 (2000). - p.96.

[130] K. Busch and S. John, Phys. Rev. E 58, 3896 (1998). — p.99.

[131] I. Nikolaev, Spontaneous Emission-Rate of Quantum Dots and Dyes Controlled with Photonic Crystals, PhD thesis, University of Twente, 2006, ISBN 90-3652414-8. - p.100 and 121.

[132] P. Jiang, J. F. Bertone, K. S. Hwang, and V. L. Colvin, Chem. Mater. 11, 2132 (1999). - p.100.

[133] J. C. Manificier, J. Gasiot, and J. P. Fillars, J. Phys. E 9, 1002 (1976). - p.101.

[134] J. E. G. J. Wijnhoven, L. Bechger, and W. L. Vos, Chem. Mater. 13, 4486 (2001). - p.101 and 102.

[135] J. Dziewior and W. Schmid, Appl. Phys. Lett. 31, 346 (1977). — p.115 and 116.

[136] J. Meyer, A. Elezzabi, and K. Y. Hughes, IEEE J. Quantum Electron. 31, 729 (1995). - p.119, 127, and 128.

[137] S. Datta, C. T. Chan, K.-M. Ho, and C. M. Soukoulis, Phys. Rev. B 48, 14936 (1993). - p.120.

[138] R. Sprik, B. A. van Tiggelen, and A. Lagendijk, Europhys. Lett. 35, 265 (1996). - p.121.

[139] Y. Akahane, T. Asano, B.-S. Song, and S. Noda, Nature 425, 944 (2003). - p.123.

[140] M. Grundmann, J. Christen, N. N. Ledentsov, J. Böhrer, D. Bimberg, S. S. 
Ruvimov, P. Werner, U. Richter, U. Gösele, J. Heydenreich, V. M. Ustinov, A. Y. Egerov, A. E. Zhukov, P. S. Kop'ev, and Z. I. Alferov, Phys. Rev. Lett. 74, 4043 (1996). - p.125.

[141] S. A. Empedocles, D. J. Norris, and M. G. Bawendi, Phys. Rev. Lett. 77 (1996). - p.125.

[142] B. L. Wehrenberg, C. Wang, and P. Guyot-Sionnest, J. Phys. Chem. B 106, 10634 (2002). - p.125.

[143] H. Du, C. Chen, R. Krishnan, T. D. Krauss, J. Harbold, F. W. Wise, M. G. Thomas, and J. Silcox, Nano Lett. 2, 1321 (2002). - p.125.

[144] M. A. Duguay and J. W. Hansen, Appl. Phys. Lett. 15, 192 (1969). — p.125.

[145] P. J. Harding, T. G. Euser, and W. L. Vos, submitted to CLEO Europe conference (2007). - p.125.

[146] H. M. van Driel, Phys. Rev. B 35, 8166 (1987). — p.127.

[147] L. Huang, J. Callan, E. N. Gleezer, and E. Mazur, Phys. Rev. Lett. 80, 185 (1998). - p.127.

[148] W. G. Spitzer and H. Y. Fan, Phys. Rev. 106, 882 (1957). — p.128.

[149] L. M. Lambert, Phys. Status Solidi A 11, 461 (1972). — p.128 and 129.

[150] M. I. Gallant and H. M. van Driel, Phys. Rev. B 26, 2133 (1982). - p.128.

[151] L. Li, J. Mod. Opt. 40, 553 (1993). - p.134.

[152] L. Li, J. Opt. Soc. Am. 13, 1024 (1996). — p.134.

[153] G. R. Fowles, Introduction to modern optics, Dover, New york, 1989. - p.136.

[154] I. Kang, T. Krauss, and F. Wise, Opt. Lett. 22, 1077 (1997). — p.139.

[155] M. Sheik-Bahae, A. A. Said, and E. W. van Stryland, Opt. Lett. 14, 955 (1989). - p.139.

[156] M. Sheik-Bahae, D. J. Hagan, and E. W. Van Stryland, Phys. Rev. Lett. 65, 96 (1990). - p.139.

[157] M. Balu, J. Hales, D. J. Hagan, and E. W. Van Stryland, Opt.. Express 16, 3840 (2004). - p.140. 


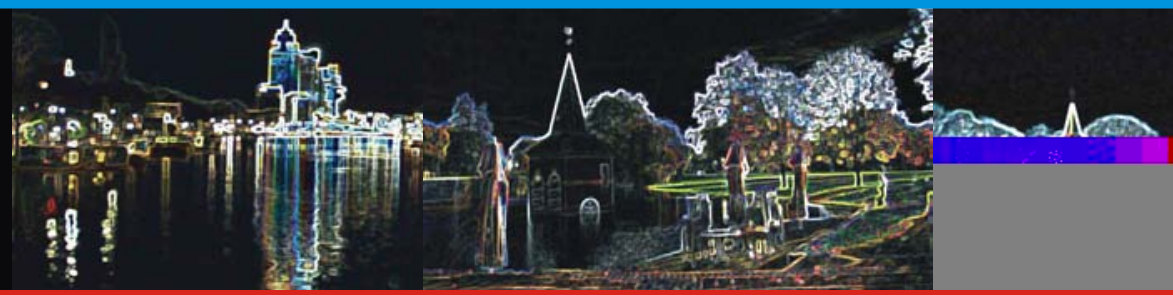

ISBN 978-90-365-2471-1 